\title{
Global, regional, and national age-sex specific all-cause and cause-specific mortality for 240 causes of death, 1990-2013: a systematic analysis for the Global Burden of Disease Study 2013
}

GBD 2013 Mortality and Causes of Death Collaborators*

\begin{abstract}
Summary
Background Up-to-date evidence on levels and trends for age-sex-specific all-cause and cause-specific mortality is essential for the formation of global, regional, and national health policies. In the Global Burden of Disease Study 2013 (GBD 2013) we estimated yearly deaths for 188 countries between 1990, and 2013. We used the results to assess whether there is epidemiological convergence across countries.
\end{abstract}

Methods We estimated age-sex-specific all-cause mortality using the GBD 2010 methods with some refinements to improve accuracy applied to an updated database of vital registration, survey, and census data. We generally estimated cause of death as in the GBD 2010. Key improvements included the addition of more recent vital registration data for 72 countries, an updated verbal autopsy literature review, two new and detailed data systems for China, and more detail for Mexico, UK, Turkey, and Russia. We improved statistical models for garbage code redistribution. We used six different modelling strategies across the 240 causes; cause of death ensemble modelling (CODEm) was the dominant strategy for causes with sufficient information. Trends for Alzheimer's disease and other dementias were informed by meta-regression of prevalence studies. For pathogen-specific causes of diarrhoea and lower respiratory infections we used a counterfactual approach. We computed two measures of convergence (inequality) across countries: the average relative difference across all pairs of countries (Gini coefficient) and the average absolute difference across countries. To summarise broad findings, we used multiple decrement life-tables to decompose probabilities of death from birth to exact age 15 years, from exact age 15 years to exact age 50 years, and from exact age 50 years to exact age 75 years, and life expectancy at birth into major causes. For all quantities reported, we computed 95\% uncertainty intervals (UIs). We constrained cause-specific fractions within each age-sex-country-year group to sum to all-cause mortality based on draws from the uncertainty distributions.

Findings Global life expectancy for both sexes increased from 65.3 years (UI 65.0-65.6) in 1990, to 71.5 years (UI 71.0-71.9) in 2013, while the number of deaths increased from 47.5 million (UI 46.8-48.2) to 54.9 million (UI 53.6-56.3) over the same interval. Global progress masked variation by age and sex: for children, average absolute differences between countries decreased but relative differences increased. For women aged 25-39 years and older than 75 years and for men aged 20-49 years and 65 years and older, both absolute and relative differences increased. Decomposition of global and regional life expectancy showed the prominent role of reductions in age-standardised death rates for cardiovascular diseases and cancers in high-income regions, and reductions in child deaths from diarrhoea, lower respiratory infections, and neonatal causes in low-income regions. HIV/AIDS reduced life expectancy in southern sub-Saharan Africa. For most communicable causes of death both numbers of deaths and age-standardised death rates fell whereas for most non-communicable causes, demographic shifts have increased numbers of deaths but decreased agestandardised death rates. Global deaths from injury increased by $10 \cdot 7 \%$, from 4.3 million deaths in 1990 to 4.8 million in 2013; but age-standardised rates declined over the same period by $21 \%$. For some causes of more than 100000 deaths per year in 2013, age-standardised death rates increased between 1990 and 2013, including HIV/AIDS, pancreatic cancer, atrial fibrillation and flutter, drug use disorders, diabetes, chronic kidney disease, and sickle-cell anaemias. Diarrhoeal diseases, lower respiratory infections, neonatal causes, and malaria are still in the top five causes of death in children younger than 5 years. The most important pathogens are rotavirus for diarrhoea and pneumococcus for lower respiratory infections. Country-specific probabilities of death over three phases of life were substantially varied between and within regions.

Interpretation For most countries, the general pattern of reductions in age-sex specific mortality has been associated with a progressive shift towards a larger share of the remaining deaths caused by non-communicable disease and injuries. Assessing epidemiological convergence across countries depends on whether an absolute or relative measure of inequality is used. Nevertheless, age-standardised death rates for seven substantial causes are increasing, suggesting the potential for reversals in some countries. Important gaps exist in the empirical data for cause of death estimates for some countries; for example, no national data for India are available for the past decade.

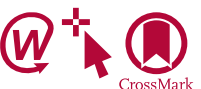
CrossMark

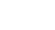

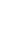




\section{Introduction}

The Global Burden of Disease (GBD) study provides a unique comprehensive framework to systematically assess national trends in age-specific and sex-specific all-cause and cause-specific mortality. Up-to-date and comprehensive evidence for levels and trends for each country is critical for informed priority setting. Trends quantify progress against explicit health targets, whether local, national, or global, and help to evaluate where programmes are working or not. Quantification across populations and over time using comparable definitions and methods can also enable benchmarking. Regular comprehensive updates about causes of death will identify emerging public health challenges. The GBD 2013 study provides the first GBD study to use a continuously updated approach to global health surveillance. ${ }^{1}$

The GBD 2010 study, a collaboration of 488 investigators, showed important global and regional trends for all-cause and cause-specific mortality. ${ }^{2-8}$ The GBD 2010 reported substantial decreases in child mortality driven by reductions in diarrhoea, lower respiratory infections, and more recently, malaria. The lowest income regions had progressed in combating maternal mortality, HIV/AIDS, tuberculosis, and malaria. Nevertheless, much work remains to be done for these Millennium Development Goal-related diseases. Outside sub-Saharan Africa, 1990-2010 saw rapid shifts towards a larger share of death from non-communicable diseases and injuries and a rising mean age of death. Country analyses using the GBD 2010 database have been reported for China, Iran, Mexico, UK, and USA, taking advantage of the comparable methods and definitions of the GBD to benchmark these countries against their peers. ${ }^{9-16}$

Much debate surrounds what should follow the

Millennium Development Goals; objective, timely, and comprehensive evidence for the levels and trends in causes of death can be a useful input. Ambitious goals have been discussed, ${ }^{16}$ such as the elimination of preventable child and maternal mortality in a generation. Targets of zero disease have been formulated for HIV/ AIDS, tuberculosis, and malaria by various groups. ${ }^{17-23}$ The Lancet Commission on Global health 2035: a world converging within a generation ${ }^{24}$ suggested that a grand convergence in health can be achieved between poor and rich countries by 2035. Advocates for non-communicable disease programmes argue ${ }^{25}$ that rapid epidemiological transitions in many regions of the world require broader health goals for the development community. Movements to focus on universal health coverage in the post-2015 health agenda emphasise the consequences of failure to meet basic health-care needs. ${ }^{2427}$

Broad interest in the GBD 2010 has led to the expansion of the GBD collaboration to include more than 1000 investigators in 106 countries. The GBD 2013 not only incorporates newly published or released datasets, particularly from the past 5 years, but also expands the analysis in other ways. We included subnational assessments for provinces of China, states of Mexico, and regions of the UK. These subnational assessments will help national decision makers to identify inequalities and local variation in leading diseases, injuries, and risk factors. The list of causes has been expanded and many new and more detailed data sources incorporated. We report the new findings for the first time at the country-level for 1990-2013.

\section{Methods \\ Study design}

The GBD approach to estimating all-cause mortality and cause-specific mortality has been previously described. ${ }^{2,3}$ Here, we describe several refinements. ${ }^{28}$ Figure 1 shows the general analysis of all-cause mortality and cause-specific mortality and their interactions. GBD 2010 included 291 causes of death or disability, of which 235 were causes of death; we have expanded the list to include 306 causes of death or disability, of which 240 are causes of death. The extra causes were added on the basis of three considerations (1) causes that were for epidemiological reasons already modelled separately but reported combined with other causes in GBD 2010-for example, silicosis, asbestosis, anorexia nervosa, and typhoid and paratyphoid fever; (2) the category of other unintentional injuries was large and heterogeneous so we broke it down further to include pulmonary aspiration and foreign body in trachea or lung, foreign body in other part of body, and unintentional suffocation; and (3) new datasets became available to enable estimation of mesothelioma, new maternal sub-causes, neonatal haemolytic anaemia, and chronic kidney disease caused by glomerulonephritis. Appendix pp 245-251 provides the International Classification of Diseases codes for the GBD 2013 cause list. After broad consultation, we have removed from the cause list the pathogen-specific causes of diarrhoeal diseases and lower respiratory infections. Instead, we analysed these causes with a counterfactual approach.

We assessed 21 regions and seven super-regions as defined in the GBD 2010. The GBD 2013 also included an assessment of subnational populations in three countries: provinces for China, states for Mexico, and the UK broken down into Scotland, Wales, Northern Ireland, and nine regions of England. We analysed these countries subnationally because of the interest from national collaborators and because sufficient data were made available by the teams in each country. In future iterations of the GBD, we hope to include further subnational breakdowns. In addition, we separately analysed data sources for rural and urban regions in India. This approach improved our estimation of mortality and causes of death and enabled us to analyse causes of death that were specific to urban or rural regions alone. 


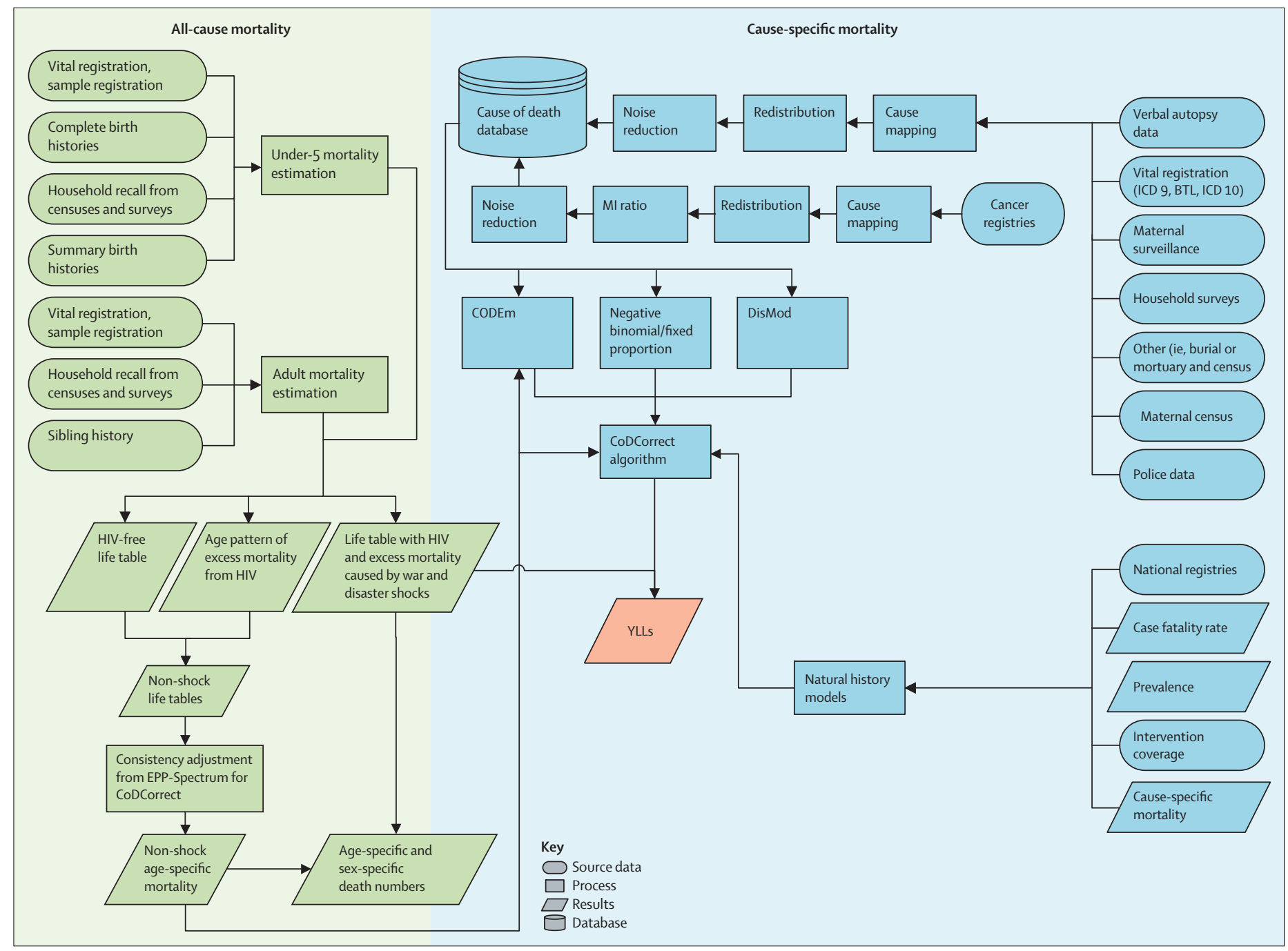

Figure 1: Components of GBD 2013 and their relations

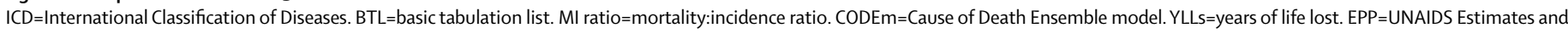
Projects Package.

\section{Covariates}

We estimated national time series (1980-2013) for a range of covariates with data from surveys (household-level and individual-level data), censuses, official reports, administrative data, and a systematic review. For lagged distributed income and education, we estimated national time series from 1950, to 2013. Details of how we imputed series for GDP, educational attainment, tobacco prevalence, and obesity prevalence have been published previously. ${ }^{29-32}$ Appendix (pp 4961-4974) shows the sources and imputation methods used to generate time series for each covariate. Generally, we estimated uncertainty in covariate values when sufficient information was available.

\section{All-cause mortality}

We analysed all-cause mortality for 188 countries from 1950 to 2013; we present only results for 1990-2013 to coincide with the period of the overall GBD 2013 assessment. As a result of the split of Sudan, we re-extracted data and made new separately generated estimates for Sudan and South Sudan. We improved how we adjusted for data source bias for the analysis of child mortality in two ways. First, by using the improved functional forms between summary mortality indicators for child (age $<5$ years) and adult (age 15-59 years) age groups and other covariates, including crude rates of death caused by HIV/AIDS. And second, by modification of the model lifetable system to use a unified standard life-table selection process and improved redistribution of excess mortality rate from HIV/AIDS. We divided the analysis into eight steps. Input data and key indicators for all countries are available online.

First, to estimate under-5 mortality $\left({ }_{5} q_{0}\right)$, we analysed all survey, census, sample registration, and vital registration sources. Wherever possible, we analysed microdata from surveys and censuses with updated methods for child mortality. ${ }^{33}$ We synthesised all measurements of under-5
For the input data and key indicators for each country see http://vizhub.healthdata.org/ mortality/ 
mortality with spatiotemporal regression and Gaussian process regression. ${ }^{33}$ We corrected for bias in different sources in specific countries.

Second, to estimate adult mortality $\left({ }_{45} q_{15}\right)$, we systematically identified all available vital registration data, sibling history survey data, sample registration data, and household recall of deaths. We assessed vital registration data for completeness by optimised death distribution methods. ${ }^{2,34}$ We analysed sibling history data to account for survivor bias, zero-surviving sibships, and recall bias. ${ }^{2,35}$ We synthesised sources with a combination of spatiotemporal regression and Gaussian process regression. The mean function for the Gaussian process regression was based on the combination of a non-linear hierarchical model with income per person, mean years of education in age group 15-60 years, mortality caused by HIV/AIDS, and country random effects ${ }^{2}$ as covariates, and a spatiotemporal regression in which we added to the first stage model without country random effects, the smoothed residuals between the first stage model and observed data (appendix pp 66-79). We selected the hyper-parameters for Gaussian process regression through an out-of-sample predictive validity testing process. ${ }^{2}$ We ranked the estimated subnational adult mortality in China, India, Mexico, and the UK to ensure that the sum of subnational estimates for a given agesex group equalled the national estimates accounting for different population sizes.

Third, we assessed HIV-free under-5 mortality and adult mortality. HIV/AIDS causes more excess mortality in younger people and thus changes the age pattern of mortality that otherwise can be readily described by Gompertz law of mortality or the Kannisto-Thatcher model. ${ }^{36,37}$ Where HIV/AIDS is common, this pattern of mortality should be explicitly taken into account. We estimated the HIV-counterfactual under-5 mortality and adult mortality rates using the estimated coefficients of crude death rate from HIV from the non-linear mixed effects models for under-5 mortality and adult mortality respectively, and setting the crude death rates from HIV/AIDS in the respective age groups to zero (appendix pp 90-94).

Fourth, we constructed an HIV-free life-table. The GBD 2010 introduced a model life-table system that used the under- 5 death rate and adult mortality rate along with a selected standard mortality schedule to estimate the full age pattern of mortality for country-years of interest. ${ }^{33}$ For GBD 2013, we modified how the standard mortality schedule was selected for each country-year so that the same approach was used for all countries. Specifically, we empirically computed a set of space-time weights that relate the observed age pattern of the probability of death in a sex-country-year with other sex-country-year observations. These weights were derived by comparing every empirical life-table that is not affected by the HIV/ AIDS epidemic in the GBD database (10673 life-tables) with every other life-table for the same sex. We estimated space-time weights as a function of the time lag between the paired life-tables and location (ie, within the country, region, or super-region). We estimated these weights as the inverse of the average sum of age-specific differences in the logit of the probability of death (appendix pp 79-91). The key observation from this spatial-temporal analysis of age-specific probabilities of death is that the mortality pattern in a country in a given year was more strongly related to the mortality pattern in the same country within 15 years than to mortality patterns in other countries; however, other countries in the same region or other regions generally are similarly related when the lag-in time was more than 20 years.

Fifth, we assessed the age pattern of HIV/AIDS mortality. Excess mortality from HIV/AIDS as quantified between the estimated ${ }_{5} q_{0}$ and ${ }_{45} q_{15}$ with their HIV counterfactual counterparts leads to increased mortality in specific age groups. This excess HIV/AIDS mortality was assigned by age with the estimated relative risk of death caused by HIV/AIDS in an age group compared with the HIV/AIDS excess death rate in age group 40-44 years. We estimated these relative risks with data from vital registration systems that have International Classification of Diseases 10 coded causes of death from HIV/AIDS, which includes South Africa. ${ }^{38}$ We used Seemingly Unrelated Regression model ${ }^{39}$ with only a constant and generated $95 \%$ uncertainty intervals (UIs) for the age pattern of relative risks by repeatedly sampling from the mean and covariance matrix of the estimated $\beta \mathrm{s}$ and the error term. Seemingly Unrelated Regression enables the error term of a series of linear regressions to be correlated. We used separate regressions by sex and for the pattern of mortality in concentrated epidemics and generalised epidemics as defined by UNAIDS..$^{38-40}$

Sixth, we minimised the difference between demographic estimation of age-specific mortality and HIV models. Murray and colleagues ${ }^{38}$ used a refined version of the EPP-Spectrum framework to model HIV/AIDS mortality. This analysis yielded very large UIs for HIV/ AIDS in many countries. However, in some southern African countries, there remained a large discrepancy between data for all-cause mortality and estimates of HIV/ AIDS mortality with demographic sources suggesting smaller epidemics. To minimise the difference between HIV/AIDS mortality and the demographic estimates, which are also uncertain, we computed a loss function that quantifies the extent to which the age-sex-country-year HIV/AIDS estimates exceed all-cause mortality:

$e_{r}=\sum_{t} \sum_{a} \sum_{s} \max \left(0, m_{r, t, a, s}^{H I V}-0.8 \times m_{r, t, a, s}^{\text {all-cause }}\right)$

For run $(r)$ of a given country, excess mortality $(e)$ is equal to the sum of all non-zero differences between HIV/AIDS mortality $\left(m^{H I V}\right)$ and 0.8 times a randomly selected all-cause mortality draw $\left(m^{\text {all-cuuse }}\right)$ across all year 
$(t)$, age $(a)$, and sex $(s)$ combinations. $0 \cdot 8$ was the highest observed cause fraction caused by HIV in any age group in any vital registration system. We selected from the uncertainty ranges for HIV and all-cause mortality those that minimised the difference. If no draws had a positive loss function, we sampled randomly from all matched draws.

The CoDCorrect algorithm includes HIV/AIDS cause-specific mortality and can alter the age and time distribution of deaths from HIV/AIDS. To incorporate the overall change in the number of HIV/AIDS deaths over the course of the epidemic in a country implied by the application of the CoDCorrect algorithm, but not to distort the Spectrum estimated time and age pattern, we adjusted the entire HIV/AIDS epidemic up or down on the basis of the cumulative effects of CoDCorrect on HIV/AIDS for all estimated years in each country.

Seventh, we used the same method as Wang and colleagues $^{2}$ to generate child mortality rate and adult mortality rate for natural disasters and armed conflicts. We obtained data for conflict and war, including deaths from one-sided violence, non-state conflict, armed force battles, and other national or international conflicts, from the Uppsala Conflict Data Program ${ }^{41}$ and the International Institute for Strategic Studies. ${ }^{42}$ Further data for war were obtained from countries' vital registration systems and classified as caused by war. ${ }^{43}$ We included disaster data from the International Disaster Database from the Center for Research on the Epidemiology of Disasters (University of Louvain, Brussels, Belgium). ${ }^{44}$ From this database, we included deaths caused by complex disaster, drought, earthquake, flood, and others. When these databases were not fully up-to-date or did not contain shocks known to exist, we supplemented with case-by-case sources. These with-shock mortality rates were then used as entry parameters to the GBD relational model life-table system to generate age-specific mortality rates with the effect of shocks added.

Eighth, we used age-specific and sex-specific death rates from the life-table to generate numbers of death by multiplying by population estimates from the World Population Prospects 2012 revision $^{45}$ and the Human Mortality Database for people older than age 5 years. For the under-5 age groups, we applied the method of Wang and colleagues. ${ }^{33}$ In some cases, assumptions in the UN estimation process led to implausible population numbers for some countries and age groups-for example, low population estimates for older age groups in South Africa, especially for the most recent years. For GBD 2013, we applied a Bayesian population reconstruction model $^{46}$ to re-estimate population for South Africa for 1970-2013.

\section{Cause of death database}

Lozano and colleagues ${ }^{3}$ described the key steps in the development of the GBD cause of death database. The database has been expanded to capture 2233 additional

\begin{tabular}{|c|c|c|c|c|c|c|}
\hline & \multicolumn{3}{|c|}{ All geographies } & \multicolumn{3}{|l|}{ GBD 2013} \\
\hline & GBD 2010 & GBD 2013 & Difference & National & $\begin{array}{l}\text { State, province, } \\
\text { or region* }\end{array}$ & Local \\
\hline Vital registration & 2798 & 5039 & $2241 \dagger$ & 2765 & 2112 & 162 \\
\hline Cancer registry & 2715 & 3860 & 1145 & 1216 & 979 & 1665 \\
\hline Sibling history & 1557 & 1798 & 241 & 1788 & 0 & 10 \\
\hline Police records & 1129 & 1433 & 304 & 1429 & 1 & 3 \\
\hline Surveillance & 128 & 1430 & 1302 & 73 & 1074 & 283 \\
\hline Verbal autopsy & 486 & 538 & 52 & 110 & 0 & 428 \\
\hline $\begin{array}{l}\text { Survey or census; hospital; } \\
\text { burial or mortuary }\end{array}$ & 154 & 146 & $-8 \ddagger$ & 94 & 0 & 52 \\
\hline Total & 8967 & 14244 & 5277 & 7475 & 4166 & 2603 \\
\hline \multicolumn{7}{|c|}{$\begin{array}{l}\text { GBD=Global Burden of Disease Study. *Data were analysed at the state level for Mexico, the province level for China, } \\
\text { and the region level for the UK. †Significant increase because of incorporation of subnational sites in China, Mexico, } \\
\text { and the UK. } \neq \text { Decrease caused by omission of World Health Survey data where adequate vital registration data was } \\
\text { available for GBD } 2013 \text {. }\end{array}$} \\
\hline
\end{tabular}

site-years of vital registration data and 52 additional verbal autopsy site-years (table 1); a site-year is defined as data for a specific geographical location (eg, a province of China) in a given year. We included data up to April 15, 2014.

A major new addition was the incorporation of two data systems in China. First, the China National Office for Maternal and Child Health Surveillance provided detailed information for child and maternal mortality by cause from 363 surveillance sites in China for 1996-2013. Second, the Disease Surveillance Points system was the main source of mortality data for 1991-2007, with 145 disease surveillance points used from 1991 to 2003, and 161 points used from 2004 to 2007. From 2008 to 2012, all of the deaths and cause of death information from the Disease Surveillance Points system and other system points throughout China were collected and reported via the Mortality Registration and Reporting System, an online reporting system of the Chinese Center for Disease Control and Prevention, which included 4.0 million deaths in 2012.47,48 Because of the discrepancy in proportions of deaths in hospital and out of hospital in the Mortality Registration and Reporting System, we divided each province in China into two strata based on the degree of urbanisation from the 2010 China Census. We then applied the proportion of deaths in hospital and out of hospital and degree of urbanisation from the Disease Surveillance Points system to the Mortality Registration and Reporting System to account for biases in the latter. We disaggregated data for both systems by province and urban and rural regions within each province. We obtained new datasets for Russia that provided more detailed causes (appendix pp 180-244). Turkey expanded its vital registration system to cover nearly all the population after 2009 and we incorporated these new data into the analysis.

In total, we identified 538 verbal autopsy site-years, 52 more than in GBD 2010. India, Bangladesh, and Tanzania had the most verbal autopsy site-years
For the Human Mortality Database see http://mortality. org/ 


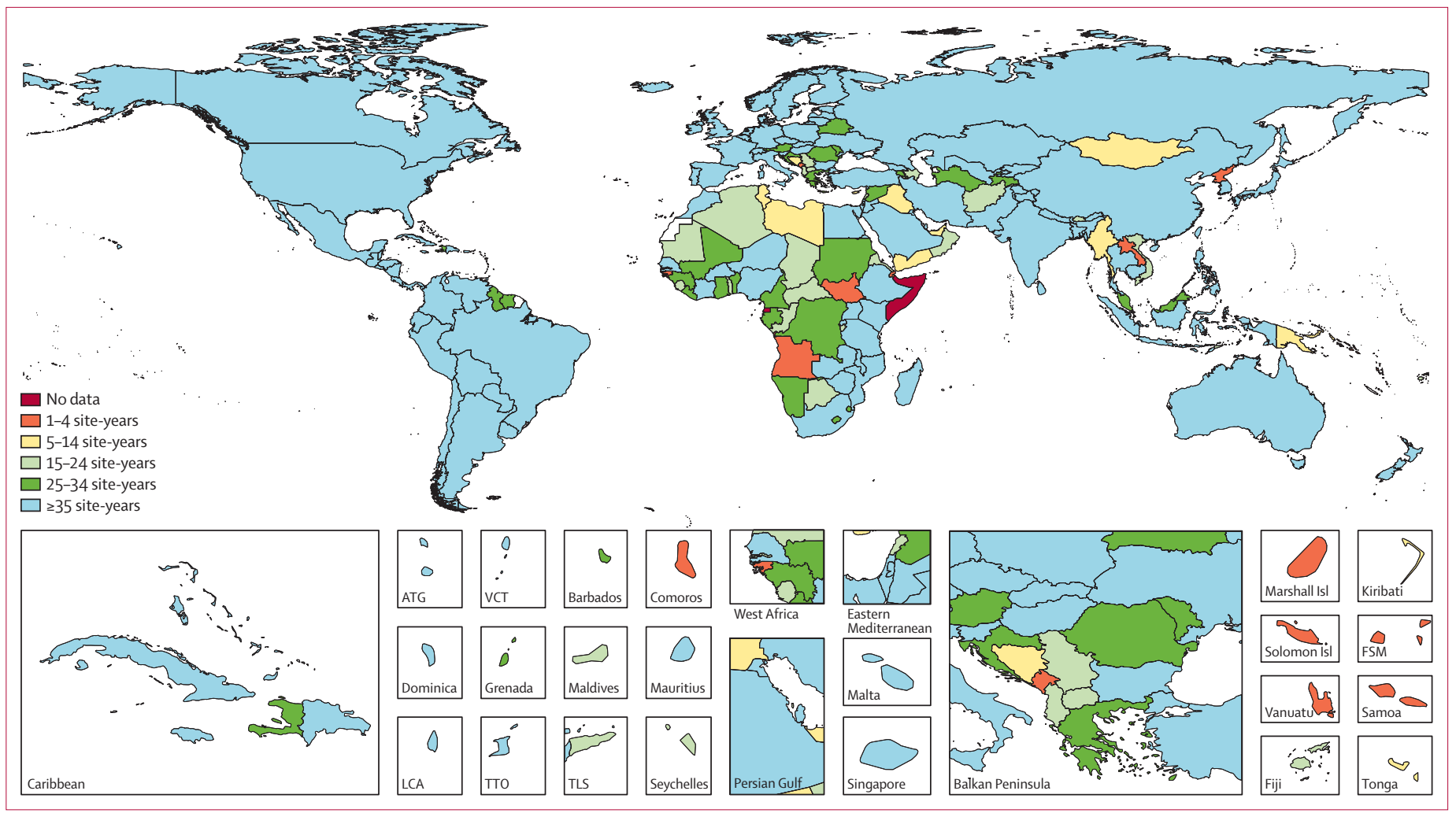

Figure 2: Site-years for all causes of death data by country, 1980-2013

ATG=Antigua and Barbuda. VCT=Saint Vincent and the Grenadines. LCA=Saint Lucia. TTO=Trinidad and Tobago. TLS=Timor-Leste. FSM=Federated States of Micronesia.

available. We re-extracted and re-mapped data from all verbal autopsy studies to ensure consistency with the GBD 2013 cause list. We excluded from the database verbal autopsy studies reporting cause assignment using InterVA because it has very low published validity. ${ }^{47} \mathrm{We}$ incorporated 1145 registry-years of new cancer data, including 128 from Cancer Incidence in Five Continents Volume $\mathrm{X}^{48}$ and 1017 from supplemental sources. Our analysis of cancer incidence and models of the death:incidence ratio remains unchanged from GBD 2010. Figure 2 shows site-years of data by country for any cause. Of note, Somalia and Equatorial Guinea had no cause of death data for any specific cause.

\section{Assessment and enhancement of quality and comparability of cause of death data}

Using the general approach of the GBD 2010, we followed six steps to assess the quality of data and enhance comparability. First, we adjusted cause of death data from vital registration systems for incompleteness. The analysis of all-cause mortality yields a separate estimate of completeness for deaths of children younger than 5 years and deaths of people older than 5 years, which we used to correct the data for cause of death. When correcting for incomplete registration, we assumed that for each age-sex-country group, the cause of death composition of registered deaths and non-registered deaths were the same. $77 \%$ of datapoints were from registration or sample registration that were more than $85 \%$ complete, $17 \%$ from systems that were $70-84 \%$ complete, and $6 \%$ were from systems less than $70 \%$ complete. Of the $6 \%$ of observations less than $70 \%$ complete, most $(62 \%)$ were for children younger than 5 years. In sensitivity tests in the GBD 2010, exclusion of data below a fixed threshold of completeness for child causes of death did not substantially change the results; thus, we have used all the data in our analysis for GBD 2013. ${ }^{3}$

Second, we developed 103 maps (excluding verbal autopsy studies) to translate causes found in the data to the GBD 2013 cause list. The expanded cause list of this study required us to adjust the maps used for data included in GBD 2010. Appendix pp 245-251 show GBD 2013 cause maps for International Classification of Diseases 9 and 10. The appendix (pp 252-253) includes more detail about changes made to the handling of various shorter tabulation lists used by some countries for reporting, such as the International Classification of Diseases 9 Basic Tabulation List.

Third, a crucial aspect of enhancing the comparability of data for cause of death is to deal with uninformative, so-called garbage codes. Garbage codes are codes for which deaths are assigned that cannot or should not be considered as the underlying cause of death-for 
example, heart failure, ill-defined cancer site, senility, ill-defined external causes of injuries, and septicaemia. Figure 3 shows the number of deaths in the database for each calendar year with the number assigned to garbage codes. Because of lags in national reporting of cause of death data, the number of deaths available after 2010 fell. Important changes for the GBD 2013 in our approach to redistributing garbage codes included the statistical estimation of the fraction of deaths following the methods outlined by Ahern and colleagues ${ }^{49}$ for deaths assigned to ill-defined cancer site, ill-defined external causes of injury, heart failure, unspecified stroke, hypertension, and atherosclerosis by region, age, and sex. Because of variation in the coding of International Classification of Diseases 10 code X59 (exposure to unspecified factor) ${ }^{50}$ and its subcauses in high-income countries, we redistributed these garbage codes with country-specific estimates for high-income countries derived from our statistical analysis. Additionally, we did not use malaria as a target for any garbage code redistribution in adults. ${ }^{51} \mathrm{We}$ also implemented geographical restrictions on garbage code redistribution for Chagas disease based on endemicity so that Chagas disease was not assigned deaths in countries outside Latin America.

Fourth, for some datasets, particularly some verbal autopsy studies, deaths were reported for broad age groups or with both sexes combined. With the addition of new data for GBD 2013, we identified 30 new age formats, totalling 112 unique age tabulations in the database. We used the algorithms described in the GBD 2010 to split these aggregated categories into estimates for specific age-sex groups.

Fifth, because few overall deaths were included in verbal autopsy studies or reported in small countries, the number of deaths by cause can fluctuate substantially from year to year. For example, in Iceland, no maternal deaths were recorded from 1991 to 2000, then one maternal death in 2001. We modified our approach to smoothing these stochastic fluctuations used in the GBD 2010 by use of a simple Bayesian algorithm. We assume a normally distributed prior and a normal data likelihood, such that:

Posterior mean $=\left(\frac{\tau^{2}}{\tau^{2}+\sigma^{2}} \mathrm{X}+\frac{\sigma^{2}}{\tau^{2}+\sigma^{2}} \mu\right)$

Posterior variance $=\left(\frac{\tau^{2} \sigma^{2}}{\tau^{2}+\sigma^{2}}\right)$

Where $X$ is the mean of the data and $\mu$ is the mean of the prior. We estimated the prior for vital registration series with a negative binomial regression with fixed effects for year and age estimated separately for each country. When the data are based on a large sample size the variance is small and the prior has little effect on the posterior. When the data have a large variance because of a small sample size, the prior has more effect, effectively borrowing strength on the age pattern from data within the same country but allowing for different levels in each

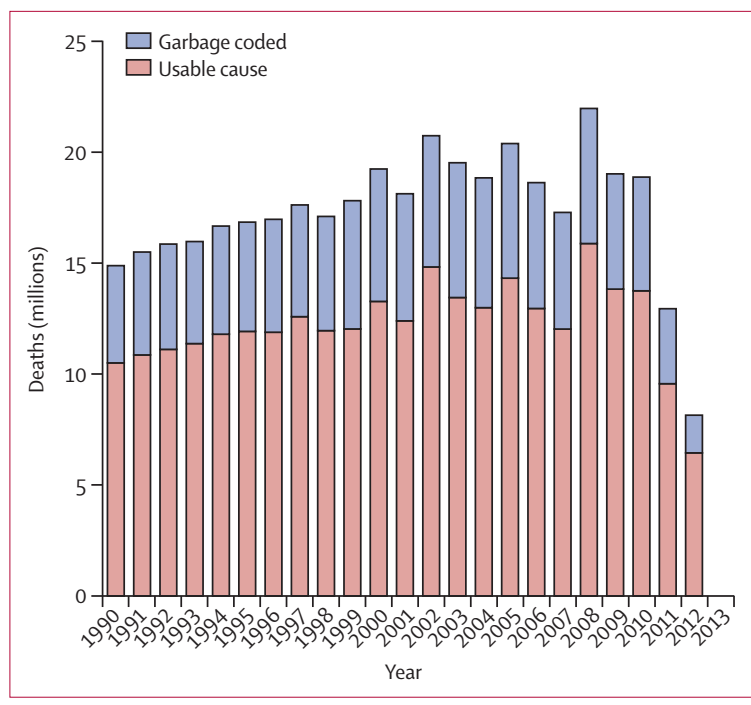

Figure 3: Total garbage and non-garbage coded deaths from vital registration and verbal autopsy sources, 1990-2013

year. For verbal autopsy studies, we modified this approach because many published reports are for a single site in a single year. The prior for each cause was based on a negative binomial with fixed effects for age groups and random effects for study-year; the regression was estimated independently for each region. For malaria, we did not group studies by region but by super-region and level of endemicity. To avoid very large negative values for log death rates or logit cause fractions, we limited the minimum non-zero posterior values to 1 per 10000000 .

Sixth, we excluded outliers based on four criteria. (1) Studies with biologically implausible values, such as $100 \%$ of mortality from a single rare cause. (2) Studies with results that were greatly inconsistent with other studies for the same country. (3) Studies that were greatly inconsistent with studies from other countries with similar sociodemographic profiles within the same region. (4) Studies that, if included, led to abrupt changes in model-estimated time trends that could not be explained by contextual changes or policy initiatives. Outliers $(0.89 \%$ of database entries) are shown in the online data visualisation of the cause of death database.

\section{Modelling individual causes of death}

As in the GBD 2010, we used six modelling strategies for causes of death depending on the strength of the available data. Where extensive data were available, we used cause of death ensemble modelling (CODEm), where fewer data were available we used simpler statistical models, and where available cause of death data might be substantially biased or not available we used natural history models (appendix pp 278-282). We generated 95\% UIs from all the modelling strategies. Uncertainty in the number of deaths for an age-sex-country-year was propagated into the computation of years of life lost (YLLs) for the same category.
For online data visualisation of the cause of death database see http://vizhub.healthdata.org/cod 
We used CODEm for 155 causes of death. CODEm has been extensively used for global health estimation including the GBD 2010., 3,52,53 An advantage is that a wide range of different models are tested; only models meeting predetermined criteria for statistical significance and direction of regression coefficients are retained. We excluded $30 \%$ of the data from the initial analysis so that the performance of different models could be assessed in terms of how well they predict the omitted data. Through multiple iterations of this process (cross-validation), we obtained stable objective information about the model's performance. The best performing models in terms of root-mean squared error for level and trend were combined into a model ensemble. For some causes, we developed separate ensemble models for GBD developed and GBD developing regions; ${ }^{54}$ the main advantage of this approach is that uncertainty in model estimation from heterogeneous data in low-income regions does not inflate the UI for high-income countries. We used this approach for all cancers and transport injuries.

For 13 causes, the number of deaths in the cause of death database was too low to generate stable estimates. For these causes, we developed negative binomial regressions with either a constant or constant multiplied by the mean assumption for the dispersion parameter, using reverse step-wise model building. We selected between the two model dispersion assumptions on the basis of best fit to the data. Compared with the GBD 2010, we modified how we generated uncertainty from these regressions by including in the uncertainty sampling draws from the $\gamma$ distribution with shape equal to the expected rate $(\mu)$ divided by expected dispersion, and scale equal to the expected dispersion if the dispersion was assumed to be constant. For models in which dispersion was assumed to equal a constant multiplied by the mean, the scale parameter included $\mu$ as a multiplicative term (instead of the shape parameter).

As in the GBD 2010, for 14 causes for which death is rare, we first modelled the parent cause in the GBD hierarchy with CODEm and then allocated deaths to specific causes using proportions of the parent cause for each sub-cause. For these causes, we identified no significant predictors in negative binomial regressions. We estimated proportions by simple averaging based on available vital registration data. Depending on the availability of data, we averaged the data across age, sex, region, and year.

We used DisMod-MR ${ }^{55}$ to estimate detailed cause fractions for several causes of death that had sufficient data to estimate proportions of a parent cause resulting from subcauses that vary across regions and countries but insufficient data to run CODEm. The source code for estimation is available online. DisMod-MR uses data for subcause fractions gathered from systematic review and from International Classification of Diseases-coded vital registration and sample registration systems. It uses two types of fixed effects (study characteristics and country covariates) with hierarchical random effects for super-region, region, and country to generate estimates for each country, age group, both sexes, and six discrete time points: 1990, 1995, 2000, 2005, 2010, and 2013. We calculated predictions for intervening years-and back to 1980 - assuming an exponential rate of change. We used this approach for eight causes of maternal death, four causes of meningitis, one cause of chronic kidney disease, four causes of cirrhosis, four causes of liver cancer, and three causes of haemoglobinopathies.

For 14 causes in the GBD 2010, we used natural history models because data systems for cause of death did not capture sufficient information. The natural history model for African trypanosomiasis was updated to include the most recent case notification data from WHO (up to 2012). We made substantial changes to the HIV natural history model..$^{25}$ Our natural history model for congenital syphilis was estimated as in the GBD 2010, with updated data for antenatal care coverage to inform the number of births at risk and additional vital registration data sources to inform age and sex distribution of deaths. We also used simple natural history models for typhoid and paratyphoid fever, whooping cough, measles, visceral leishmaniasis, and yellow fever. Additionally, because vital registration data recording the specific type of hepatitis were very sparse, we used natural history models for all the detailed causes of hepatitis. The natural history model takes into account the extensive serological data for the prevalence of antibodies or antigens for hepatitis A, B, and C, and more limited data for case-fatality rates.

Alzheimer's disease and other dementias were analysed with CODEm in GBD 2010. Because of the large inconsistency between the data for prevalence and mortality, we used a natural history model in the GBD 2013. Prevalences have not changed substantially over time, whereas age-standardised mortality rates in high-income countries have increased, ranging from about 25\% (Denmark, Switzerland, Norway) to $46 \%$ (Germany). The prevalence of dementia varies between countries by a factor of three, whereas dementia mortality recorded in vital registration data and verbal autopsy studies varies by more than 20 -fold. On the basis of these findings, we believe that the variation in dementia mortality rates between countries and over time was probably affected by changes in coding practices with increased propensity to assign dementia as an underlying cause of death. To correct for this, we assessed data from 23 high-income countries with high-quality vital registration systems to estimate the ratio of registered dementia deaths:prevalent cases. In DisMod-MR, we used the mean of these ratios as an estimate of excess mortality to estimate age-specific and sex-specific mortality from dementia consistent with the meta-regression of prevalence.

In GBD 2010, because single-cause models were developed for each cause, the final step was to combine 
these models into estimates that are consistent with allcause mortality for each age-sex-country-year group. For each cause-specific model and for all-cause mortality, we had 1000 draws from the posterior distribution for each age-sex-country-year group. We combined causes by taking a random draw without replacement from the posterior distribution of each cause and all-cause mortality. Each cause was rescaled by a scalar equal to the draw of all-cause mortality divided by the sum of the draws of individual causes. The GBD 2010 induced a correlation of 1.0 between the sum of cause-specific and all-cause mortality. CoDCorrect was applied in a hierarchical fashion: first to level 1 causes and then to level 2 and level 3 causes. Level 2 causes were constrained to sum to the level 1 parent cause. Levels of this cascade were largely the same as those used in the GBD 2010 and were chosen on the basis of the amount and quality of available data for cause of death.

For GBD 2013, we made slight modifications to this approach. Because tests showed no substantial effect of the 1.0 correlation between draws of all-cause mortality and the sum of individual causes and because each cause is modelled independently such that the ordering of draws across causes were unrelated, we have removed this assumption. Furthermore, because the modelling of HIV through the GBD version of Spectrum uses relationships between incidence, CD4 progression, and death that are age-dependent and antiretroviral therapy scale-up over time has had major effects, we modified the way in which HIV deaths are handled in CoDCorrect. We ran CoDCorrect for all causes and then computed the pre-CoDCorrect cumulative deaths over time and age and compared with the cumulative deaths post-CoDCorrect. This provided an overall scalar, which we used to adjust the entire HIV epidemic. To avoid in any age-sex-country-year the sum of individual deaths exceeding all-cause mortality, we computed the difference between the cumulatively scaled HIV deaths and the CoDCorrect HIV deaths and added this difference to the estimate of all-cause mortality at the draw level.

In GBD 2010, diarrhoea deaths and lower respiratory infection deaths were reported for pathogen-specific causes in tabulations that summed to $100 \%$ of each parent cause. Since the GBD 2010, the GEMS study ${ }^{56}$ has been published, which provided data for the relative risk of diarrhoea being related to different pathogens. This relative risk approach used a different conceptual framework than did the International Classification of Diseases approach for underlying cause. Underlying cause follows the more than 200-year history of health statistics of assigning each death uniquely to a single underlying cause. The relative risk approach follows the approach used more generally for risk factors, where cause is assigned based on comparison to a counterfactual. Counterfactual attribution to specific risks or in this case pathogens, can sum to more or less than $100 \%$. On the basis of the GEMS study and consultations among experts in both diarrhoea and lower respiratory infection, we report results for counterfactual causes in GBD 2013. To estimate diarrhoea mortality attributable to different pathogens, we calculated the population attributable fraction for pathogens including rotavirus, Shigella, enteropathogenic Escherichia coli, enterotoxigenic E coli, adenovirus (enteric adenovirus), norovirus, Aeromonas, other Salmonella (non-typhoidal Salmonella), Cryptosporidium, Campylobacter, and Entamoeba. We used the Miettinen formula, which uses the distribution of pathogens in patients and relative risks of pathogens for diarrhoea, to provide a population attributable fraction for each pathogen: ${ }^{57,58}$

$$
P A F_{i}=p_{i}(\text { pathogen in patients })\left(1-\frac{1}{\text { odds ratio }_{i}}\right)
$$

Where $P A F_{i}$ is the population attributable fraction of diarrhoea caused by pathogen $i, p_{i}$ is the prevalence of pathogen $i$ in patients with diarrhoea, and odds ratio is the odds ratio of diarrhoea in people with the pathogen. We used DisMod-MR to estimate the proportion of patients in each age-sex-country-year with each pathogen with data from studies of inpatients and community samples. By use of study-level covariates in the meta-regression, we obtained different estimates for inpatients and community samples. We assumed inpatients to be a proxy for severe diarrhoea and death. We reanalysed GEMS ${ }^{59}$ to estimate the odds ratio for each pathogen in a multipathogen model by conditional logistic regression. Regression models included fixed effects for a specific pathogen with interaction terms for three age groups (0-1 years, 1-2 years, and 2-6 years) to allow different odds ratios by age and interaction terms for different GEMS field sites (Bangladesh, India, Kenya, Mali, Mozambique, Pakistan, and The Gambia) to estimate site-specific odds ratios. For other countries in the region, the odds ratio we used was the average of the odds ratios (in logarithm scale) of the countries with GEMS sites in that region. For countries in central and southern subSaharan Africa, we used the average of GEMS sites located in eastern and western sub-Saharan Africa. For all other countries in regions without GEMS sites, we used the average of all odds ratio. To produce odds ratio uncertainty while averaging odds ratio, we generated 1000 draws of joint normal distribution using a covariance matrix from the conditional logistic regression for each of the GEMS countries in a region. To produce the draws for non-GEMS countries, we selected draws from each of the GEMS countries until we had a full set of 1000 draws. For example, to generate 1000 draws for countries in eastern sub-Saharan Africa, we used draws from Kenya and Mozambique-the two GEMS countries within that region. We pulled 500 of the Kenya draws and 500 of the Mozambique draws to produce our full set of 
1000 draws, which were used for all the other countries in this region. Because GEMS included only diarrhoea in children younger than 5 years, we applied the odds ratio of pathogens calculated for children aged 2-5 years when calculating for adults. We did not assign diarrhoea cases or deaths to a pathogen for an age-country-year if more than $95 \%$ of draws were greater than 1 .

For cholera, we used data from previous studies (appendix p 310) and compared them with WHO case notification data to estimate under-reporting for cholera and then the number of cases (appendix $p$ 310). To estimate cholera deaths, we modelled cholera case fatality in DisMod-MR with data from previous studies.

Clostridium difficile as a cause of diarrhoea in children is rarely studied; we could not estimate the epidemiological population attributable fraction as we did for other pathogens because $C$ difficile was not included in GEMS. Because $C$ difficile is related to hospital and health-care use, we used hospital data as the primary source for estimation. We modelled the incidence and case fatality of $C$ difficile and assumed a 1-month risk of death ${ }^{60}$ in DisMod-MR to estimate the number of deaths.

For GBD 2013, we split lower respiratory infection mortality into four categories: Streptococcus pneumoniae, Haemophilus influenzae type B pneumonia, respiratory syncytial virus pneumonia, and influenza. The counterfactual approach captures the complex interactions between the these causes ${ }^{61}$ and also excludes the "other lower respiratory infection" category included in GBD 2010. Moreover, we did not attribute lower respiratory infection to any cause for children younger than age 1 month. We adopted a different approach to estimate bacterial and viral causes on the basis of available data. For pneumococcal and $H$ influenzae type B pneumonia, we estimated the causal fraction from vaccine efficacy studies. ${ }^{62-64}$ For pneumococcal pneumonia, we included data from controlled trials and observational studies, such as before-after population analyses of the introduction of pneumococcal vaccine. ${ }^{65-76}$ For $H$ influenzae type $\mathrm{B}$, we excluded case-control studies because of implausibly large estimates of vaccine efficacy. Furthermore, unlike for pneumococcal vaccine, little data were available from vaccine efficacy studies on the effect outside of child ages. As a result, we did not estimate the causal fraction of $H$ influenzae type B for lower respiratory infection in people aged 5 years or older. We adjusted data for efficacy, using invasive disease as a marker as well as serotype coverage for pneumococcal vaccine. ${ }^{64}$ We calculated pooled estimates of causal fractions by age with DisMod-MR for pneumococcal vaccine and random-effects meta-analysis for $H$ influenzae type $\mathrm{B}$, adjusted post-hoc for national-level coverage of pneumococcal vaccine and $H$ influenzae type $B$ vaccine. For respiratory syncytial virus and influenza, we relied on observational studies that measured causal fractions among hospital admissions for lower respiratory infection. We estimated the causal fractions among cases by country, age, and sex with DisMod-MR and the odds ratio of exposure from case-control studies. To account for the higher case-fatality of bacterial versus viral lower respiratory infections, we applied a relative case-fatality differential based on in-hospital case-fatality using hospital admissions that included cases coded to the specific pneumonia causes.

\section{Convergence measures}

To test whether all-cause and cause-specific mortality converged in the 188 countries since 1990 , we computed two measures: the average relative difference and the average absolute difference between any pair of countries included in the GBD 2013 study. The average relative difference is known as the Gini coefficient and is the most commonly used measure of inequality. For international comparisons, we used the population-weighted version of the Gini coefficient in age-specific mortality rates so that small populations do not have an undue influence on the assessment of global mortality convergence (appendix pp 556-557). ${ }^{\text {7 }}$ For the Gini coefficient to fall, the percent decrease in mortality for countries with higher mortality must in general be faster than that for countries with lower mortality.

We also computed the mean absolute difference for all-cause mortality for each age group for 1990-2013 and for age-standardised rates for each cause (appendix pp 556-557). Average absolute difference can fall while average relative difference (the Gini coefficient) rises. The two measures provide different perspectives on convergence.

\section{Multiple decrement lifetables}

We used age-specific cause of death and all-cause mortality life-tables to compute the conditional probability of death for three summary intervals: childhood and adolescence (0 to exact age 15 years), reproductive-age adults ( 15 years to exact age 50 years), middle-aged adults ( 50 years to exact age 75 years), and the cause-specific contributions to each of these summary indicators. For each conditional probability of death, we used the multiple decrement life-table method $^{78}$ to compute the probability of death from each cause and the overall contribution of each cause of death to the summary probability of death indicators for the three broad age groups (appendix pp 556-557). We calculated the decomposition of changes in life expectancy by age and cause of death as detailed by Beltran-Sanchez, Preston, and Canudas-Romo. ${ }^{79}$

\section{Age-standardised rates and YLLs}

For GBD 2010, we computed age-standardised mortality rates and YLL rates from the world population age standard issued by WHO in $2001{ }^{80}$ To account for the substantial change in global demographics since 2001, we updated this standard. We used the same method as 
WHO and computed a standard population structure with population estimates for 2010-35 from the most recent World Population Prospects by the United Nations Population Division. Appendix pp 95-96 provides details of the GBD world population agestandard. We computed YLLs by multiplying numbers of deaths from each cause in each age group by the reference life expectancy at the average age of death for those who die in the age group following the standard GBD 2010 methods. $^{3}$ The appendix (pp 121-40) shows key indicators from the new GBD standard life-table.

\section{Ranking lists and decomposition analysis}

We used the GBD 2010 approach to create ranked lists of specific diseases and injuries. We modified GBD 2010 ranking list to incorporate newly estimated causes with the same overall assignment of rank causes as GBD 2010: typhoid and paratyphoid separately, haemolytic disease in fetus and newborn and other neonatal jaundice, mesothelioma, unintentional suffocation, pulmonary aspiration and foreign body in trachea or lung, and foreign body in other part of body. Following the methods developed by Lozano and colleagues, ${ }^{3}$ we decomposed changes in the number of global deaths and global YLLs into the contributions from population growth, population aging, and agespecific death rates.

\section{Role of the funding source}

The funder of the study had no role in study design, data collection, data analysis, data interpretation, or writing of the report. The authors had access to the data in the study and the final responsibility to submit the paper.

\section{Results}

\section{Global all-cause mortality}

Global life expectancy at birth for both sexes increased from 65.3 years in 1990 , to 71.5 years in 2013 , an average increase of 0.27 years per calendar year. Life expectancy increased over this period by 6.6 years for females and 5.8 years for males. Figure 4 shows the yearly change in global life expectancy at birth, with a large drop in the 1990s as a result of the Rwanda genocide and famine in North Korea and the return to increases of about 0.3 years or more per year since 2003. If the median rate of change of the last 23 years continues, by 2030 global female life expectancy will be $85 \cdot 3$ years and male life expectancy will be $78 \cdot 1$ years. Reduced fertility and the consequent demographic shift of the world's population to older ages has led to the mean age of death increasing from 46.7 years in 1990 , to 59.3 years in $2013 .^{81}$

The number of deaths globally for both sexes all ages increased from 47.47 (UI 46.77-48.22) million in 1990, to $54 \cdot 86(53 \cdot 57-56 \cdot 33)$ million in 2013 , partly because of consistent increases in global population over the past

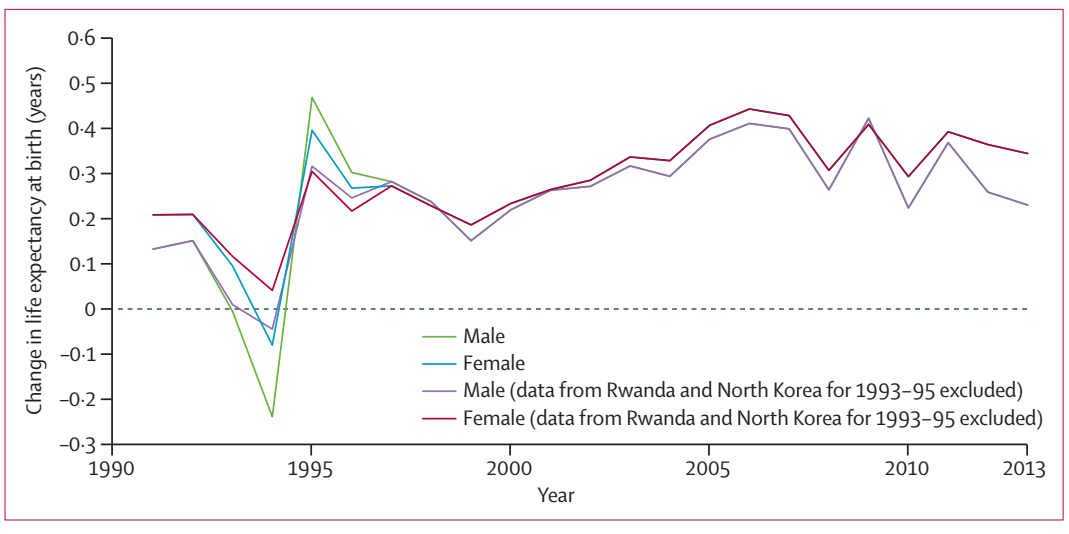

Figure 4: Change in global life expectancy at birth for males and females

decades. Rapid falls in child death rates compared with other age-specific death rates have led to a shift in the age structure of global deaths with substantial decreases in children and large increases in the proportion of deaths of people older than age 80 years (figure 5). The number of child deaths fell between 1990 and 2013 in southeast Asia, east Asia, and Oceania with very substantial falls in north Africa and the Middle East, and Latin America and the Caribbean (figure 5). However, the number of child deaths in sub-Saharan Africa only changed from 3.68 $(3 \cdot 63-3 \cdot 73)$ million in 1990 , to $3 \cdot 20(3 \cdot 00-3 \cdot 42)$ million in 2013. Substantial increases in the number of deaths of people older than age 80 years have occurred in high-income regions as well as in southeast Asia, east Asia, and Oceania.

Rising global life expectancy at birth has not come from uniform progress across age-groups or countries. In all age-groups except the 80 years and older age group, mean mortality rate has decreased more for females than for males (figure 6). Larger decreases in males older than age 80 years might be a result of the differences in the age composition between males and females in this openended age group. The mortality rate in the under- 5 age group has fallen much more between 1990 and 2013 than has that for older age groups. The smallest decreases occurred in men in age groups 30-34 years, 35-39 years, and 80 years or older, and in women aged 80 years or older.

For all age groups, population-weighted average relative difference for age-specific mortality rates differences across countries (ie, inequality) increased except in age group 10-14 years and 15-19 years for females. The divergence in age-specific mortality rates was greatest in young adult age groups between ages 20 years and 44 years for both males and females; dominant causes in these age groups include HIV/AIDS, interpersonal violence, maternal mortality, and road injury (data not shown). For many age groups, in both sexes, the absolute differences have fallen while relative inequalities have increased (figure 6). For women aged 25-39 years and 75 years and older, and for men aged 20-49 years and 65 years and 


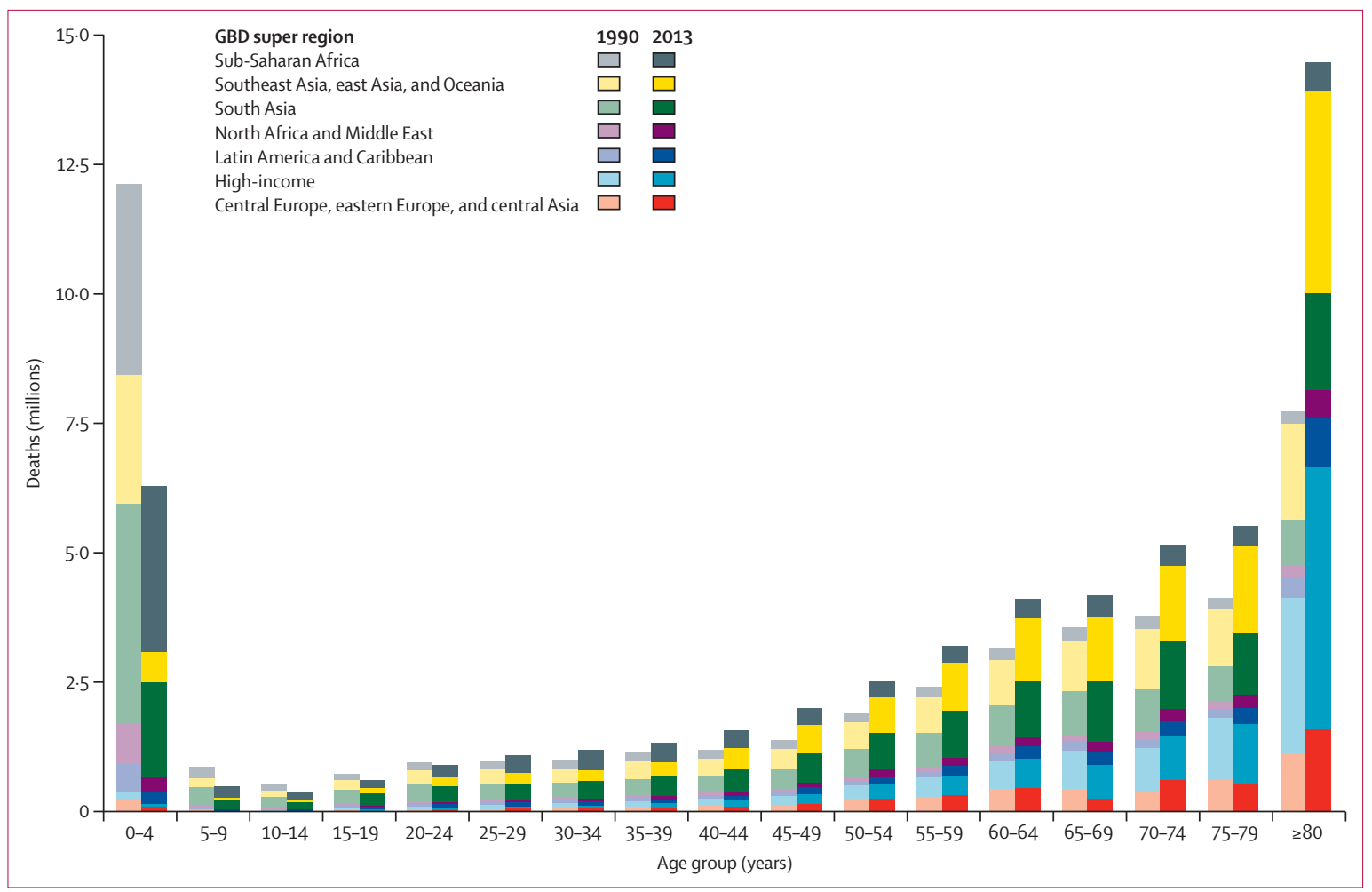

Figure 5: Global deaths by age and super region in 1990 and 2013

older, both relative and absolute differences in mean agespecific mortality rates have increased since 1990.

\section{Global causes of death}

We decomposed change in global and regional life expectancy by cause (level 2 of the GBD cause hierarchy; figure 7). Increased life expectancy since 1990 was mainly caused by a fall in mortality from lower respiratory infections and diarrhoeal diseases (contributing 2.2 years), cardiovascular and circulatory diseases (contributing $1 \cdot 1$ years), neonatal conditions (contributing 0.7 years), cancers (contributing 0.4 years), and chronic respiratory diseases (contributing 0.5 years). Decreases in mortality from unintentional injuries added another 0.3 years to life expectancy, while female life expectancy increased by about 0.2 years because of reductions in maternal mortality. These gains were offset by increased mortality from diabetes, chronic kidney diseases, and related conditions, as well as musculoskeletal disorders, although the net effect of these increases was small, reducing life expectancy, on average, by about $0 \cdot 1$ years. Five main causes reduced life expectancy: HIV/AIDS was a major cause of death in southern sub-Saharan Africa and to a smaller extent in western and eastern subSaharan Africa; diabetes, chronic kidney disease, and other endocrine disorders decreased life expectancy across many regions, most notably in Oceania and central Latin America; mental disorders made a negative contribution in multiple regions, especially high-income north America; intentional injuries reduced life expectancy in south Asia, high-income Asia Pacific, and southern sub-Saharan Africa; and cirrhosis made a negative impact in eastern Europe and central Asia (figure 7). Large gains in life expectancy in sub-Saharan Africa were mainly driven by reductions of diarrhoea and lower respiratory infections and of neonatal disorders. Gains in high-income regions were driven by reductions in cardiovascular disease, some cancers, transport injuries, and chronic respiratory conditions (figure 7).

Between 1990 and 2013, numbers of deaths from non-communicable diseases and injuries steadily increased while deaths from communicable, maternal, neonatal, and nutritional causes decreased (table 2). However, age-standardised rates decreased in these three broad categories. The shift to non-communicable diseases, at least at globally, was driven by faster rates of decline for communicable, maternal, neonatal and nutritional causes and an ageing world population.

In 2013, $11 \cdot 8$ million (11.3-12.3) deaths were caused by communicable, maternal, neonatal, and nutritional disorders: $2 \cdot 7$ million $(2 \cdot 4-2 \cdot 8)$ by lower respiratory infections, $1 \cdot 3$ million $(1 \cdot 3-1 \cdot 5)$ by HIV/AIDS, $1 \cdot 3$ million $(1 \cdot 2-1 \cdot 4)$ by tuberculosis, and $1 \cdot 3$ million $(1 \cdot 2-1 \cdot 4)$ by diarrhoeal diseases, $2 \cdot 0$ million $(1 \cdot 9-2 \cdot 2)$ by neonatal conditions, 854600 (702924-1032497) by malaria, and 293336 (261322-328200) by maternal causes (about 20\% 


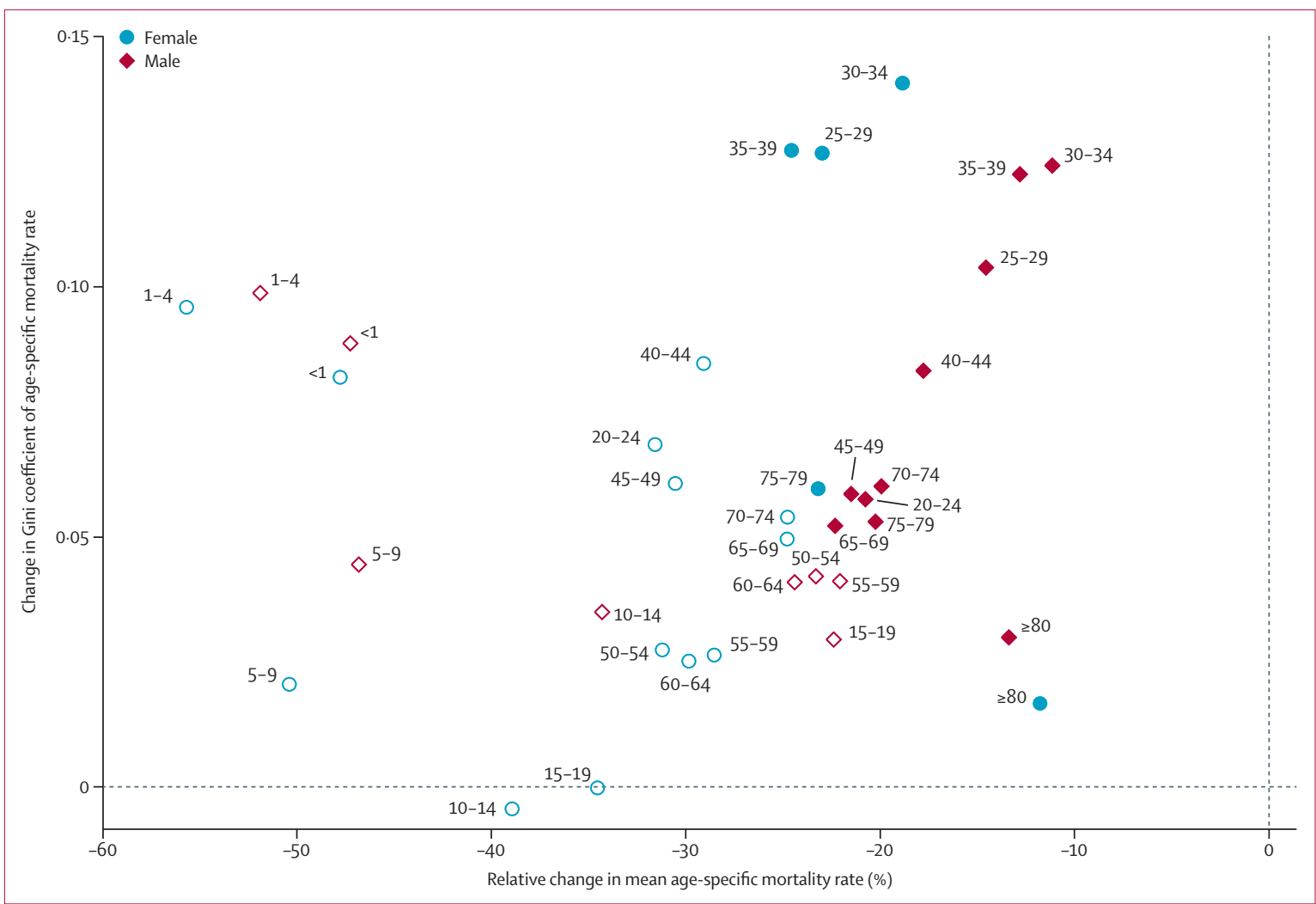

Figure 6: Change in age-specific population-weighted Gini coefficient versus relative change in mortality rate from 1990 to 2013

Solid points show age groups for which the mean absolute difference between countries has increased and hollow points show those for which mean absolute difference has decreased.

less than in 2000). Between 2000 and 2013, deaths from diarrhoeal diseases fell by $31.1 \%$ (from 1.8 million $[1 \cdot 7-2 \cdot 0]$ to $1 \cdot 3$ million $[1 \cdot 2-1 \cdot 4])$, tuberculosis and meningitis each by about $20 \%(1.6$ million $[1 \cdot 4-1 \cdot 7]$ to 1.3 million [1.2-1.4] for tuberculosis, 377300 [331400-438000] to 303500 [261400-346300] for meningitis), while mortality from tetanus fell by about 60\% (142400 [108800-163100] to 58900 [39800-77300]), from measles by about $80 \% \quad(494500 \quad[279.4-763 \cdot 8]$ to 95600 [48500-172900]), from diphtheria by about 40\% (5400 [2800-10700] to 3300 [1700-6600]), and from whooping cough by about $40 \%$ (111800 [42 300-242400] to 60600 [22300-136800]). Deaths from neonatal causes fell by a quarter since 2000 (2.8 million to 2.0 million), and by about one-fifth for maternal causes (364900 to 293300). Comparing 2013 to 1990, malaria deaths decreased by $4.4 \%$ and HIV/AIDS increased by $368 \%$ (table 2). HIV/ AIDS mortality and malaria mortality both peaked in 2005 (1.7 million [1.6-1.9] for HIV/AIDS, 1.2 million [1.1-1.4] for malaria); HIV/AIDS mortality fell by $21 \%(20 \cdot 4-21 \cdot 5)$ from 2005 to 2013, and malaria mortality fell by $30 \%$ $(24 \cdot 8-35 \cdot 4))^{38}$ The risk of death from various leading causes of communicable, maternal, neonatal, and nutritional disorders as measured by the age-standardised death rate (table 2), has generally declined by an even greater amount than the risk for HIV/AIDS and malaria.
Age-standardised death rates decreased by about $40 \%$ since 1990 for the category as a whole, as well as most notably, for lower respiratory infections, maternal disorders, neonatal disorders, and asthma, and by $50-60 \%$ for tuberculosis, diarrhoeal diseases, pneumoconiosis and several neglected tropical diseases (table 2). Despite a small decrease in numbers of deaths, age-standardised malaria mortality have fallen by $19 \%$ since 1990 , with much of that decline occurring in the past 5 years or so (data not shown). ${ }^{33}$

For most of the leading non-communicable diseases, the number of deaths has increased, by $42 \%$ between 1990 and 2013 (from 27.0 million [UI 26.3-27.6] in 1990, to $38 \cdot 3$ million [37.2-39.4] in 2013), but age-standardised mortality rates have fallen. Allowing for changes in the age structure of the world's population between 1990 and 2013, age-standardised death rates from non-communicable diseases fell by $18.6 \%$; by $22 \%$ for cardiovascular and circulatory diseases, $13 \cdot 7-14 \cdot 7 \%$ for cirrhosis of the liver and cancer, and $21 \cdot 9-30 \cdot 4 \%$ for other digestive diseases and chronic respiratory diseases (table 2). For many disorders, including stomach cancer, Hodgkin lymphoma, rheumatic heart disease, peptic ulcer disease, appendicitis, and schizophrenia, agestandardised death rates have fallen by more than onethird since 1990 (table 2). Age-standardised death rates 


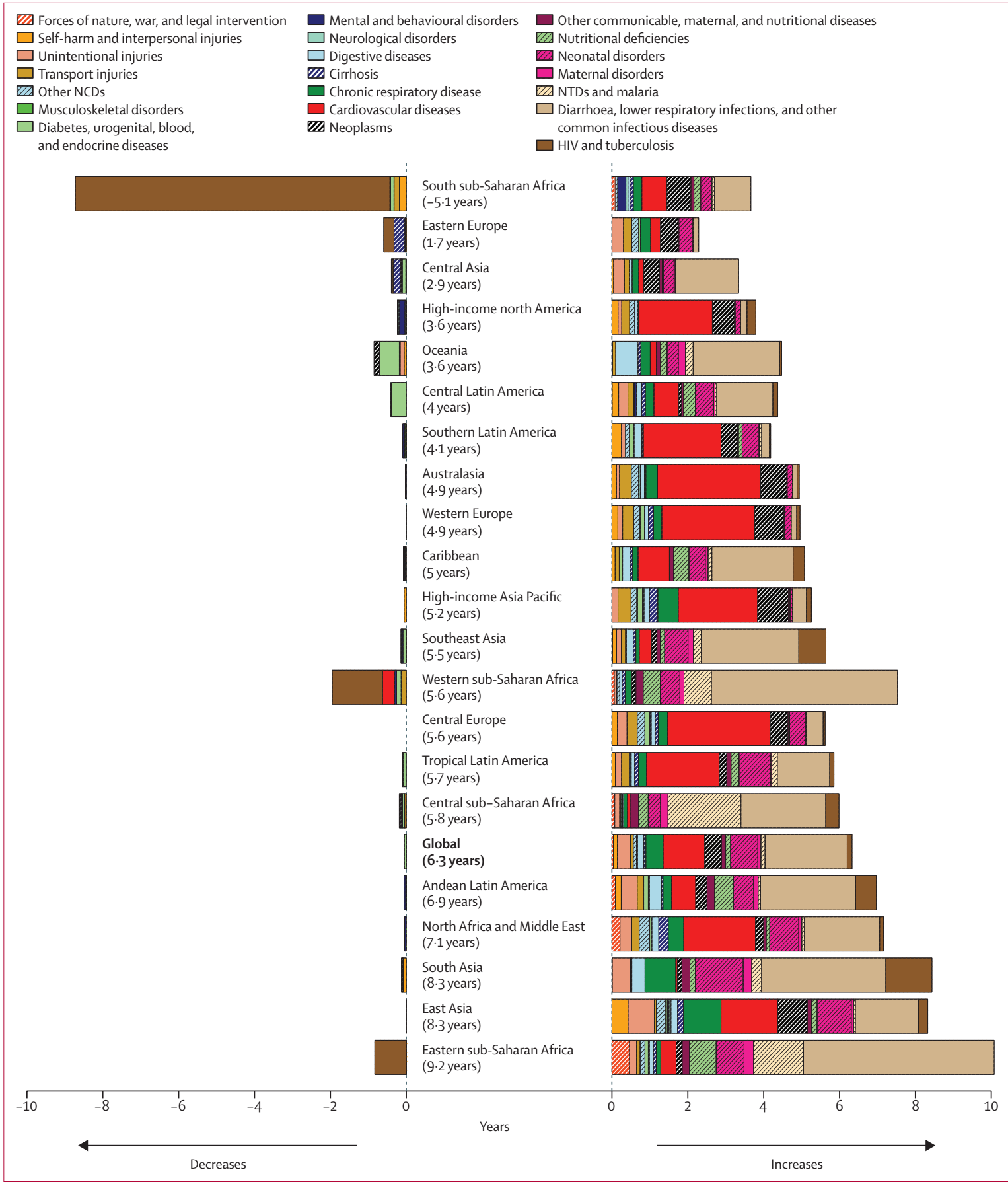

Figure 7: Change in life expectancy at birth by GBD region and cause group from 1990 to 2013

An interactive figure with these data is available at http://vizhub.healthdata.org/le. Changes in life expectancy as a result of specific causes were decomposed from the difference between all-cause lifetables and cause-deleted lifetables (mortality set to zero for a specific cause). Because all changes in life expectancy are based on cross-sectional lifetables, the cause-specific changes add up to the total change in life-expectancy. NTDs=neglected tropical diseases.

for some cancers have fallen (lung by $9 \%$, breast by $18 \%$, and leukaemia by $20 \%$ ), but have remained unchanged for others (table 2). Global age-standardised death rates have fallen by more than one-fifth for ischaemic heart disease and stroke (table 2).
Global age-standardised mortality rates increased significantly for very few disease between 1990 and 2013. The largest increase was for HIV/AIDS, which peaked in 2005 and then fell by $31.0 \%$ (UI $25 \cdot 7 \%$ to $35 \cdot 9 \%$ ) from 2005 to 2013 (from $26 \cdot 9$ to $18 \cdot 5$ per 100000 ). Among the 


\begin{tabular}{|c|c|c|c|c|c|c|}
\hline & \multicolumn{3}{|c|}{ All ages deaths (thousands) } & \multicolumn{3}{|c|}{ Age-standardised death rate (per 100000) } \\
\hline & 1990 & 2013 & Median \% change & 1990 & 2013 & Median \% change \\
\hline All causes & $\begin{array}{l}47468 \cdot 7 \\
(46771 \cdot 7 \text { to } 48223 \cdot 3)\end{array}$ & $\begin{array}{l}54863 \cdot 8 \\
(53576 \cdot 1 \text { to } 56333 \cdot 6)\end{array}$ & $\begin{array}{l}15 \cdot 6 \\
(12 \cdot 54 \text { to } 19 \cdot 11)\end{array}$ & $\begin{array}{l}1160 \cdot 5 \\
(1143 \cdot 2 \text { to } 1179 \cdot 0)\end{array}$ & $\begin{array}{l}879.7 \\
(859 \cdot 9 \text { to } 902 \cdot 2)\end{array}$ & $\begin{array}{l}-24 \cdot 2 \\
(-26 \cdot 20 \text { to }-21 \cdot 96)\end{array}$ \\
\hline $\begin{array}{l}\text { Communicable, maternal, neonatal, } \\
\text { and nutritional diseases }\end{array}$ & $\begin{array}{l}16149 \cdot 4 \\
(15674 \cdot 5 \text { to } 16597 \cdot 6)\end{array}$ & $\begin{array}{l}11809 \cdot 6 \\
(11335 \cdot 5 \text { to } 12283 \cdot 0)\end{array}$ & $\begin{array}{l}-26 \cdot 8 \\
(-29 \cdot 99 \text { to }-24 \cdot 10)\end{array}$ & $\begin{array}{l}289 \cdot 5 \\
(281 \cdot 2 \text { to } 298 \cdot 4)\end{array}$ & $\begin{array}{l}172 \cdot 2 \\
(165 \cdot 2 \text { to } 178 \cdot 8)\end{array}$ & $\begin{array}{l}-40 \cdot 5 \\
(-42 \cdot 98 \text { to }-38 \cdot 35)\end{array}$ \\
\hline HIV/AIDS and tuberculosis & $\begin{array}{l}2072 \cdot 5 \\
(1938 \cdot 2 \text { to } 2246 \cdot 1)\end{array}$ & $\begin{array}{c}2631 \cdot 2 \\
(2497 \cdot 3 \text { to } 2863 \cdot 2)\end{array}$ & $\begin{array}{l}26.8 \\
(17.08 \text { to } 37.92)\end{array}$ & $\begin{array}{c}49 \cdot 3 \\
(46 \cdot 2 \text { to } 53 \cdot 3)\end{array}$ & $\begin{array}{l}38 \cdot 0 \\
(36 \cdot 0 \text { to } 41 \cdot 6)\end{array}$ & $\begin{array}{l}-23 \cdot 1 \\
(-29 \cdot 02 \text { to }-16 \cdot 39)\end{array}$ \\
\hline Tuberculosis & $\begin{array}{c}1786 \cdot 1 \\
(1666 \cdot 4 \text { to } 1945 \cdot 4)\end{array}$ & $\begin{array}{c}1290 \cdot 3 \\
(1167 \cdot 3 \text { to } 1406 \cdot 2)\end{array}$ & $\begin{array}{l}-27 \cdot 7 \\
(-34 \cdot 99 \text { to }-20 \cdot 56)\end{array}$ & $\begin{array}{c}43 \cdot 5 \\
(40 \cdot 4 \text { to } 47 \cdot 2)\end{array}$ & $\begin{array}{c}19 \cdot 4 \\
(17 \cdot 6 \text { to } 21 \cdot 2)\end{array}$ & $\begin{array}{l}-55 \cdot 3 \\
(-59 \cdot 67 \text { to }-51 \cdot 01)\end{array}$ \\
\hline HIV/AIDS & $\begin{array}{c}286 \cdot 4 \\
(227 \cdot 4 \text { to } 370 \cdot 3)\end{array}$ & $\begin{array}{l}1341 \cdot 0 \\
(1257 \cdot 8 \text { to } 1482 \cdot 6)\end{array}$ & $\begin{array}{l}374 \cdot 2 \\
(262 \cdot 47 \text { to } 490 \cdot 73)\end{array}$ & $\begin{array}{c}5 \cdot 8 \\
(4 \cdot 6 \text { to } 7 \cdot 6)\end{array}$ & $\begin{array}{l}18 \cdot 5 \\
(17 \cdot 4 \text { to } 20 \cdot 5)\end{array}$ & $\begin{array}{l}222 \cdot 0 \\
(145 \cdot 14 \text { to } 303 \cdot 34)\end{array}$ \\
\hline $\begin{array}{l}\text { HIV/AIDS resulting in mycobacterial } \\
\text { infection }\end{array}$ & $\begin{array}{c}27 \cdot 8 \\
(20 \cdot 5 \text { to } 37 \cdot 4)\end{array}$ & $\begin{array}{c}84 \cdot 0 \\
(67 \cdot 4 \text { to } 104 \cdot 9)\end{array}$ & $\begin{array}{l}205 \cdot 1 \\
(130 \cdot 68 \text { to } 291 \cdot 83)\end{array}$ & $\begin{array}{c}0.6 \\
(0.4 \text { to } 0.8)\end{array}$ & $\begin{array}{c}1.2 \\
(0.9 \text { to } 1 \cdot 4)\end{array}$ & $\begin{array}{l}105 \cdot 5 \\
(55 \cdot 00 \text { to } 166 \cdot 22)\end{array}$ \\
\hline $\begin{array}{l}\text { HIV/AIDS resulting in other } \\
\text { diseases }\end{array}$ & $\begin{array}{c}258 \cdot 6 \\
(206 \cdot 1 \text { to } 334 \cdot 4)\end{array}$ & $\begin{array}{c}1257 \cdot 0 \\
\text { (1178.1 to } 1391 \cdot 1)\end{array}$ & $\begin{array}{l}393 \cdot 2 \\
(276 \cdot 86-511 \cdot 66)\end{array}$ & $\begin{array}{c}5 \cdot 3 \\
(4 \cdot 2 \text { to } 6 \cdot 8)\end{array}$ & $\begin{array}{c}17 \cdot 4 \\
(16 \cdot 3 \text { to } 19 \cdot 2)\end{array}$ & $\begin{array}{l}235 \cdot 0 \\
\text { (154.55 to } 319 \cdot 43)\end{array}$ \\
\hline $\begin{array}{l}\text { Diarrhoea, lower respiratory infections, } \\
\text { and other common infectious diseases }\end{array}$ & $\begin{array}{l}7880 \cdot 5 \\
(7468 \cdot 3 \text { to } 8337 \cdot 2)\end{array}$ & $\begin{array}{l}4750 \cdot 5 \\
(4388 \cdot 8 \text { to } 5029 \cdot 9)\end{array}$ & $\begin{array}{l}-39 \cdot 4 \\
(-45 \cdot 17 \text { to }-35 \cdot 89)\end{array}$ & $\begin{array}{l}143 \cdot 7 \\
(137 \cdot 3 \text { to } 151 \cdot 9)\end{array}$ & $\begin{array}{c}72 \cdot 4 \\
(66 \cdot 7 \text { to } 76 \cdot 5)\end{array}$ & $\begin{array}{l}-49 \cdot 4 \\
(-53 \cdot 54 \text { to }-46 \cdot 75)\end{array}$ \\
\hline Diarrhoeal diseases & $\begin{array}{l}2578 \cdot 7 \\
(2412 \cdot 2 \text { to } 2748 \cdot 9)\end{array}$ & $\begin{array}{c}1264 \cdot 1 \\
(1151 \cdot 2 \text { to } 1383 \cdot 2)\end{array}$ & $\begin{array}{l}-51 \cdot 0 \\
(-55 \cdot 55 \text { to }-46 \cdot 25)\end{array}$ & $\begin{array}{c}47 \cdot 4 \\
(44 \cdot 4 \text { to } 50 \cdot 1)\end{array}$ & $\begin{array}{l}19 \cdot 0 \\
(17 \cdot 4 \text { to } 20 \cdot 8)\end{array}$ & $\begin{array}{l}-59.8 \\
(-63.54 \text { to }-55.96)\end{array}$ \\
\hline Intestinal infectious diseases & $\begin{array}{c}259 \cdot 1 \\
(145 \cdot 6 \text { to } 424 \cdot 6)\end{array}$ & $\begin{array}{c}221 \cdot 3 \\
(122 \cdot 6 \text { to } 362 \cdot 6)\end{array}$ & $\begin{array}{l}-14 \cdot 3 \\
(-26.08 \text { to }-2 \cdot 75)\end{array}$ & $\begin{array}{c}4 \cdot 3 \\
(2 \cdot 4 \text { to } 7 \cdot 0)\end{array}$ & $\begin{array}{c}3 \cdot 1 \\
(1 \cdot 7 \text { to } 5 \cdot 0)\end{array}$ & $\begin{array}{l}-28.7 \\
(-38.19 \text { to }-18.98)\end{array}$ \\
\hline Typhoid fever & $\begin{array}{c}180 \cdot 5 \\
(96 \cdot 4 \text { to } 302 \cdot 3)\end{array}$ & $\begin{array}{c}160 \cdot 7 \\
(85 \cdot 9 \text { to } 268 \cdot 0)\end{array}$ & $\begin{array}{l}-10 \cdot 8 \\
(-23 \cdot 70 \text { to } 4 \cdot 24)\end{array}$ & $\begin{array}{c}3 \cdot 0 \\
(1 \cdot 6 \text { to } 5 \cdot 0)\end{array}$ & $\begin{array}{c}2 \cdot 2 \\
(1 \cdot 2 \text { to } 3 \cdot 7)\end{array}$ & $\begin{array}{l}-25 \cdot 9 \\
(-36 \cdot 31 \text { to }-13 \cdot 57)\end{array}$ \\
\hline Paratyphoid fever & $\begin{array}{c}63 \cdot 4 \\
(33 \cdot 6 \text { to } 106 \cdot 7)\end{array}$ & $\begin{array}{c}54 \cdot 3 \\
(29 \cdot 3 \text { to } 92 \cdot 0)\end{array}$ & $\begin{array}{l}-14 \cdot 9 \\
(-30 \cdot 51 \text { to } 9 \cdot 48)\end{array}$ & $\begin{array}{c}1.0 \\
(0.6 \text { to } 1 \cdot 7)\end{array}$ & $\begin{array}{c}0.7 \\
(0.4 \text { to } 1 \cdot 3)\end{array}$ & $\begin{array}{l}-28.0 \\
(-40.99 \text { to }-7.97)\end{array}$ \\
\hline Other intestinal infectious diseases & $\begin{array}{c}15 \cdot 3 \\
(13 \cdot 5 \text { to } 17 \cdot 3)\end{array}$ & $\begin{array}{c}6 \cdot 3 \\
(5 \cdot 5 \text { to } 7 \cdot 0)\end{array}$ & $\begin{array}{l}-59 \cdot 1 \\
(-63.26 \text { to }-54 \cdot 09)\end{array}$ & $\begin{array}{c}0.3 \\
(0.2 \text { to } 0 \cdot 3)\end{array}$ & $\begin{array}{c}0 \cdot 1 \\
(0 \cdot 1 \text { to } 0 \cdot 1)\end{array}$ & $\begin{array}{l}-66 \cdot 7 \\
(-70 \cdot 02 \text { to }-62 \cdot 84)\end{array}$ \\
\hline Lower respiratory infections & $\begin{array}{c}3420 \cdot 7 \\
(3211.6 \text { to } 3638 \cdot 4)\end{array}$ & $\begin{array}{c}2652.6 \\
\text { (2368.0 to 2808.1) }\end{array}$ & $\begin{array}{l}-22 \cdot 2 \\
(-29 \cdot 71 \text { to }-16 \cdot 32)\end{array}$ & $\begin{array}{c}66 \cdot 8 \\
(63.1 \text { to } 71 \cdot 6)\end{array}$ & $\begin{array}{c}41 \cdot 7 \\
(37 \cdot 1 \text { to } 44 \cdot 1)\end{array}$ & $\begin{array}{l}-37 \cdot 4 \\
(-42 \cdot 54 \text { to }-33 \cdot 45)\end{array}$ \\
\hline Upper respiratory infections & $\begin{array}{c}4.7 \\
(4.0 \text { to } 5 \cdot 6)\end{array}$ & $\begin{array}{c}3 \cdot 9 \\
(3 \cdot 3 \text { to } 4 \cdot 7)\end{array}$ & $\begin{array}{l}-16 \cdot 4 \\
(-33 \cdot 55 \text { to } 4 \cdot 22)\end{array}$ & $\begin{array}{c}0 \cdot 1 \\
(0 \cdot 1 \text { to } 0 \cdot 1)\end{array}$ & $\begin{array}{c}0 \cdot 1 \\
(0 \cdot 1 \text { to } 0 \cdot 1)\end{array}$ & $\begin{array}{l}-40 \cdot 8 \\
(-52 \cdot 40 \text { to }-26 \cdot 16)\end{array}$ \\
\hline Otitis media & $\begin{array}{c}4 \cdot 9 \\
(4 \cdot 5 \text { to } 5 \cdot 3)\end{array}$ & $\begin{array}{c}2 \cdot 4 \\
(2 \cdot 3 \text { to } 2 \cdot 6)\end{array}$ & $\begin{array}{l}-50 \cdot 8 \\
(-54 \cdot 22 \text { to }-46 \cdot 41)\end{array}$ & $\begin{array}{c}0.1 \\
(0 \cdot 1 \text { to } 0 \cdot 1)\end{array}$ & $\begin{array}{c}0.0 \\
(0.0 \text { to } 0.0)\end{array}$ & $\begin{array}{l}-60 \cdot 8 \\
(-63 \cdot 34 \text { to }-57 \cdot 76)\end{array}$ \\
\hline Meningitis & $\begin{array}{c}464 \cdot 4 \\
(405 \cdot 0 \text { to } 559 \cdot 0)\end{array}$ & $\begin{array}{c}303 \cdot 5 \\
(261 \cdot 4 \text { to } 346 \cdot 3)\end{array}$ & $\begin{array}{l}-34 \cdot 3 \\
(-45 \cdot 34 \text { to }-24 \cdot 08)\end{array}$ & $\begin{array}{c}7 \cdot 7 \\
(6 \cdot 8 \text { to } 9 \cdot 0)\end{array}$ & $\begin{array}{c}4 \cdot 3 \\
(3 \cdot 7 \text { to } 4 \cdot 9)\end{array}$ & $\begin{array}{l}-43.9 \\
(-52.65 \text { to }-36.07)\end{array}$ \\
\hline Pneumococcal meningitis & $\begin{array}{c}112 \cdot 1 \\
(97 \cdot 7 \text { to } 132 \cdot 6)\end{array}$ & $\begin{array}{c}79 \cdot 1 \\
(67 \cdot 8 \text { to } 91 \cdot 1)\end{array}$ & $\begin{array}{l}-29.4 \\
(-39.84 \text { to }-16.86)\end{array}$ & $\begin{array}{c}1.9 \\
(1 \cdot 7 \text { to } 2 \cdot 2)\end{array}$ & $\begin{array}{c}1 \cdot 1 \\
(1 \cdot 0 \text { to } 1 \cdot 3)\end{array}$ & $\begin{array}{l}-41 \cdot 7 \\
(-49 \cdot 60 \text { to }-31 \cdot 37)\end{array}$ \\
\hline $\begin{array}{l}\text { Haemophilus influenzae type B } \\
\text { meningitis }\end{array}$ & $\begin{array}{c}118 \cdot 0 \\
(98 \cdot 2 \text { to } 147 \cdot 0)\end{array}$ & $\begin{array}{c}64 \cdot 4 \\
(53 \cdot 0 \text { to } 76 \cdot 4)\end{array}$ & $\begin{array}{l}-45 \cdot 4 \\
(-54 \cdot 79 \text { to }-33 \cdot 50)\end{array}$ & $\begin{array}{c}1 \cdot 8 \\
(1 \cdot 5 \text { to } 2 \cdot 2)\end{array}$ & $\begin{array}{c}0.9 \\
(0 \cdot 7 \text { to } 1 \cdot 1)\end{array}$ & $\begin{array}{l}-49 \cdot 7 \\
(-58 \cdot 21 \text { to }-39 \cdot 32)\end{array}$ \\
\hline Meningococcal meningitis & $\begin{array}{c}88 \cdot 1 \\
(76 \cdot 1 \text { to } 108 \cdot 0)\end{array}$ & $\begin{array}{c}65 \cdot 7 \\
(55 \cdot 9 \text { to } 75 \cdot 8)\end{array}$ & $\begin{array}{l}-24.9 \\
(-38.82 \text { to }-12.62)\end{array}$ & $\begin{array}{c}1 \cdot 5 \\
(1 \cdot 3 \text { to } 1 \cdot 8)\end{array}$ & $\begin{array}{c}0.9 \\
(0.8 \text { to } 1 \cdot 1)\end{array}$ & $\begin{array}{l}-37 \cdot 3 \\
(-47 \cdot 55 \text { to }-27 \cdot 80)\end{array}$ \\
\hline Other meningitis & $\begin{array}{c}146 \cdot 1 \\
(128 \cdot 0 \text { to } 174 \cdot 9)\end{array}$ & $\begin{array}{c}94 \cdot 2 \\
(82 \cdot 2 \text { to } 106 \cdot 4)\end{array}$ & $\begin{array}{l}-35 \cdot 1 \\
(-47 \cdot 16 \text { to }-24 \cdot 71)\end{array}$ & $\begin{array}{c}2 \cdot 5 \\
(2 \cdot 2 \text { to } 2 \cdot 9)\end{array}$ & $\begin{array}{c}1 \cdot 3 \\
(1 \cdot 2 \text { to } 1 \cdot 5)\end{array}$ & $\begin{array}{l}-45 \cdot 9 \\
(-54 \cdot 98 \text { to }-37 \cdot 48)\end{array}$ \\
\hline Encephalitis & $\begin{array}{c}92 \cdot 2 \\
(65 \cdot 2 \text { to } 116 \cdot 2)\end{array}$ & $\begin{array}{c}77 \cdot 3 \\
(65 \cdot 4 \text { to } 97 \cdot 0)\end{array}$ & $\begin{array}{l}-15 \cdot 2 \\
(-40 \cdot 15 \text { to } 16 \cdot 01)\end{array}$ & $\begin{array}{c}1.6 \\
(1.1 \text { to } 1 \cdot 9)\end{array}$ & $\begin{array}{c}1.1 \\
(0.9 \text { to } 1.4)\end{array}$ & $\begin{array}{l}-28 \cdot 7 \\
(-48 \cdot 10 \text { to }-4 \cdot 52)\end{array}$ \\
\hline Diphtheria & $\begin{array}{c}8 \cdot 0 \\
(3 \cdot 9 \text { to } 18 \cdot 8)\end{array}$ & $\begin{array}{c}3 \cdot 3 \\
(1.7 \text { to } 6 \cdot 6)\end{array}$ & $\begin{array}{l}-57 \cdot 7 \\
(-85 \cdot 61 \text { to } 12 \cdot 54)\end{array}$ & $\begin{array}{c}0.1 \\
(0 \cdot 1 \text { to } 0 \cdot 3)\end{array}$ & $\begin{array}{c}0.0 \\
(0.0 \text { to } 0 \cdot 1)\end{array}$ & $\begin{array}{l}-60 \cdot 8 \\
(-86 \cdot 70 \text { to } 2 \cdot 82)\end{array}$ \\
\hline Whooping cough & $\begin{array}{c}138 \cdot 2 \\
(52 \cdot 9 \text { to } 300 \cdot 2)\end{array}$ & $\begin{array}{c}60 \cdot 6 \\
(22 \cdot 3 \text { to } 136 \cdot 8)\end{array}$ & $\begin{array}{l}-56 \cdot 7 \\
(-83 \cdot 76 \text { to } 14 \cdot 31)\end{array}$ & $\begin{array}{c}1.9 \\
(0 \cdot 7 \text { to } 4 \cdot 2)\end{array}$ & $\begin{array}{c}0.8 \\
(0.3 \text { to } 1.9)\end{array}$ & $\begin{array}{l}-58 \cdot 2 \\
(-84 \cdot 31 \text { to } 10 \cdot 44)\end{array}$ \\
\hline Tetanus & $\begin{array}{c}356 \cdot 2 \\
(292 \cdot 9 \text { to } 578 \cdot 6)\end{array}$ & $\begin{array}{c}58 \cdot 9 \\
(39 \cdot 8 \text { to } 77 \cdot 3)\end{array}$ & $\begin{array}{l}-82 \cdot 1 \\
(-92 \cdot 00 \text { to }-76 \cdot 10)\end{array}$ & $\begin{array}{c}5 \cdot 7 \\
(4 \cdot 7 \text { to } 9 \cdot 1)\end{array}$ & $\begin{array}{c}0.8 \\
(0.6 \text { to } 1 \cdot 1)\end{array}$ & $\begin{array}{l}-83.9 \\
(-92 \cdot 72 \text { to }-77 \cdot 84)\end{array}$ \\
\hline & & & & & & 2 continues on nex \\
\hline
\end{tabular}

cancers, only liver cancer caused by hepatitis C increased substantially (table 2). Although age-standardised mortality for cardiovascular and circulatory diseases decreased by $22 \%$, significant increases occurred for atrial fibrillation and flutter and peripheral vascular disease (table 2). Mortality rates for Alzheimer's disease and other dementias increased by only $3 \cdot 2 \%$ (UI $-3 \cdot 01$ to $11 \cdot 61)$ and Parkinson's disease by $28 \cdot 2 \%(-6.42$ to 37.83; table 2). Important worldwide increases occurred for diabetes $(9.0 \%)$ and an even larger increase for chronic kidney disease (36.9\%; table 2). The agestandardised death rate for sickle-cell disease increased 


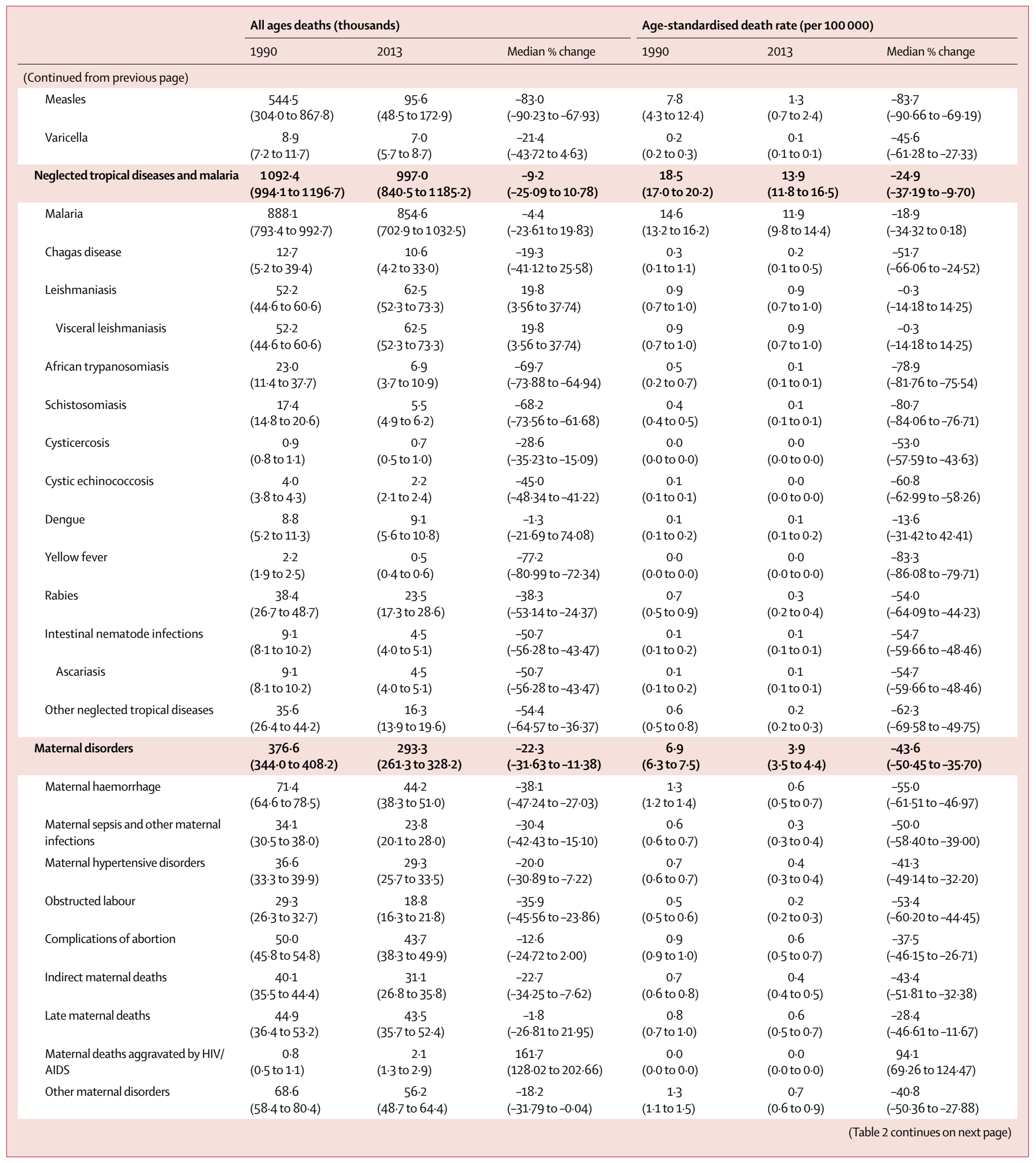




\begin{tabular}{|c|c|c|c|c|c|c|}
\hline & \multicolumn{3}{|c|}{ All ages deaths (thousands) } & \multicolumn{3}{|c|}{ Age-standardised death rate (per 100000 ) } \\
\hline & 1990 & 2013 & Median \% change & 1990 & 2013 & Median \% change \\
\hline \multicolumn{7}{|l|}{ (Continued from previous page) } \\
\hline Neonatal disorders & $\begin{array}{l}3433 \cdot 3 \\
(3225 \cdot 7 \text { to } 3586 \cdot 1)\end{array}$ & $\begin{array}{l}2048 \cdot 0 \\
(1934 \cdot 7 \text { to } 2160 \cdot 3)\end{array}$ & $\begin{array}{l}-40 \cdot 3 \\
(-43 \cdot 96 \text { to }-36 \cdot 30)\end{array}$ & $\begin{array}{c}46 \cdot 9 \\
(44 \cdot 1 \text { to } 49 \cdot 0)\end{array}$ & $\begin{array}{l}27 \cdot 6 \\
(26 \cdot 1 \text { to } 29 \cdot 1)\end{array}$ & $\begin{array}{l}-41 \cdot 1 \\
(-44 \cdot 71 \text { to }-37 \cdot 13)\end{array}$ \\
\hline Preterm birth complications & $\begin{array}{l}1570 \cdot 5 \\
(1285 \cdot 0 \text { to } 1803 \cdot 5)\end{array}$ & $\begin{array}{c}742 \cdot 4 \\
\text { (591.3 to } 910 \cdot 8)\end{array}$ & $\begin{array}{l}-52 \cdot 9 \\
(-58 \cdot 70 \text { to }-45 \cdot 50)\end{array}$ & $\begin{array}{c}21 \cdot 4 \\
(17 \cdot 5 \text { to } 24 \cdot 6)\end{array}$ & $\begin{array}{c}10 \cdot 0 \\
(8 \cdot 0 \text { to } 12 \cdot 3)\end{array}$ & $\begin{array}{l}-53 \cdot 5 \\
(-59 \cdot 23 \text { to }-46 \cdot 20)\end{array}$ \\
\hline $\begin{array}{l}\text { Neonatal encephalopathy (birth } \\
\text { asphyxia/trauma) }\end{array}$ & $\begin{array}{c}874 \cdot 1 \\
(688 \cdot 5 \text { to } 1055 \cdot 4)\end{array}$ & $\begin{array}{c}643 \cdot 8 \\
(515 \cdot 0 \text { to } 760 \cdot 5)\end{array}$ & $\begin{array}{l}-26 \cdot 1 \\
(-38 \cdot 29 \text { to }-11 \cdot 35)\end{array}$ & $\begin{array}{c}11 \cdot 9 \\
(9 \cdot 4 \text { to } 14 \cdot 4)\end{array}$ & $\begin{array}{c}8 \cdot 7 \\
(6 \cdot 9 \text { to } 10 \cdot 3)\end{array}$ & $\begin{array}{l}-27 \cdot 1 \\
(-39 \cdot 06 \text { to }-12 \cdot 49)\end{array}$ \\
\hline $\begin{array}{l}\text { Neonatal sepsis and other neonatal } \\
\text { infections }\end{array}$ & $\begin{array}{c}346 \cdot 4 \\
(195 \cdot 7 \text { to } 484 \cdot 0)\end{array}$ & $\begin{array}{c}366 \cdot 0 \\
(233 \cdot 2 \text { to } 510 \cdot 8)\end{array}$ & $\begin{array}{c}6 \cdot 1 \\
(-15 \cdot 94 \text { to } 38 \cdot 04)\end{array}$ & $\begin{array}{c}4 \cdot 7 \\
(2 \cdot 7 \text { to } 6 \cdot 6)\end{array}$ & $\begin{array}{c}4.9 \\
(3 \cdot 1 \text { to } 6 \cdot 9)\end{array}$ & $\begin{array}{l}4.6 \\
(-17 \cdot 13 \text { to } 35 \cdot 99)\end{array}$ \\
\hline $\begin{array}{l}\text { Haemolytic disease and other neonatal } \\
\text { jaundice }\end{array}$ & $\begin{array}{c}64 \cdot 8 \\
(39 \cdot 5 \text { to } 96 \cdot 3)\end{array}$ & $\begin{array}{c}19 \cdot 6 \\
(13 \cdot 0 \text { to } 29 \cdot 7)\end{array}$ & $\begin{array}{l}-70 \cdot 0 \\
(-79 \cdot 78 \text { to }-50 \cdot 43)\end{array}$ & $\begin{array}{c}0.9 \\
(0.5 \text { to } 1 \cdot 3)\end{array}$ & $\begin{array}{c}0.3 \\
(0.2 \text { to } 0 \cdot 4)\end{array}$ & $\begin{array}{l}-70 \cdot 6 \\
(-80 \cdot 17 \text { to }-51 \cdot 17)\end{array}$ \\
\hline Other neonatal disorders & $\begin{array}{c}577 \cdot 6 \\
(457.5 \text { to } 756 \cdot 0)\end{array}$ & $\begin{array}{c}276 \cdot 2 \\
(219 \cdot 6 \text { to } 350 \cdot 7)\end{array}$ & $\begin{array}{l}-52 \cdot 3 \\
(-62 \cdot 41 \text { to }-36 \cdot 77)\end{array}$ & $\begin{array}{c}7 \cdot 9 \\
(6 \cdot 3 \text { to } 10 \cdot 3)\end{array}$ & $\begin{array}{c}3 \cdot 7 \\
(3 \cdot 0 \text { to } 4 \cdot 7)\end{array}$ & $\begin{array}{l}-53 \cdot 0 \\
(-63 \cdot 01 \text { to }-37 \cdot 68)\end{array}$ \\
\hline Nutritional deficiencies & $\begin{array}{c}757 \cdot 7 \\
(641 \cdot 5 \text { to } 934 \cdot 4)\end{array}$ & $\begin{array}{c}681 \cdot 1 \\
(533 \cdot 5 \text { to } 795 \cdot 5)\end{array}$ & $\begin{array}{l}-9.7 \\
(-22.87 \text { to } 1.86)\end{array}$ & $\begin{array}{c}14 \cdot 7 \\
(12 \cdot 4 \text { to } 18 \cdot 2)\end{array}$ & $\begin{array}{c}10 \cdot 4 \\
(8 \cdot 2 \text { to } 12 \cdot 2)\end{array}$ & $\begin{array}{l}-28 \cdot 6 \\
(-38 \cdot 77 \text { to }-20 \cdot 45)\end{array}$ \\
\hline Protein-energy malnutrition & $\begin{array}{c}507 \cdot 9 \\
(394 \cdot 3 \text { to } 648 \cdot 5)\end{array}$ & $\begin{array}{c}468 \cdot 8 \\
(350 \cdot 0 \text { to } 560 \cdot 9)\end{array}$ & $\begin{array}{c}-7.3 \\
(-22.92 \text { to } 7.51)\end{array}$ & $\begin{array}{c}9 \cdot 2 \\
(7 \cdot 1 \text { to } 11 \cdot 8)\end{array}$ & $\begin{array}{c}7 \cdot 1 \\
(5 \cdot 3 \text { to } 8 \cdot 5)\end{array}$ & $\begin{array}{l}-22 \cdot 5 \\
(-34 \cdot 54 \text { to }-11 \cdot 34)\end{array}$ \\
\hline lodine deficiency & $\begin{array}{c}2 \cdot 1 \\
(1.4 \text { to } 3 \cdot 4)\end{array}$ & $\begin{array}{c}2.7 \\
\text { (1.5 to } 4.7)\end{array}$ & $\begin{array}{l}24.5 \\
(-29.82 \text { to } 137 \cdot 21)\end{array}$ & $\begin{array}{c}0.0 \\
(0.0 \text { to } 0 \cdot 1)\end{array}$ & $\begin{array}{c}0.0 \\
(0.0 \text { to } 0.1)\end{array}$ & $\begin{array}{l}-7 \cdot 9 \\
(-47 \cdot 13 \text { to } 80 \cdot 62)\end{array}$ \\
\hline Iron-deficiency anemia & $\begin{array}{c}213 \cdot 4 \\
(143 \cdot 5 \text { to } 309 \cdot 3)\end{array}$ & $\begin{array}{c}183 \cdot 4 \\
(122 \cdot 0 \text { to } 259 \cdot 2)\end{array}$ & $\begin{array}{l}-13 \cdot 8 \\
(-32 \cdot 36 \text { to } 5 \cdot 33)\end{array}$ & $\begin{array}{c}4 \cdot 6 \\
(3 \cdot 3 \text { to } 6 \cdot 6)\end{array}$ & $\begin{array}{c}2.8 \\
(1.9 \text { to } 4 \cdot 0)\end{array}$ & $\begin{array}{l}-38 \cdot 8 \\
(-50 \cdot 36 \text { to }-26 \cdot 57)\end{array}$ \\
\hline Other nutritional deficiencies & $\begin{array}{c}34 \cdot 3 \\
(23 \cdot 8 \text { to } 56 \cdot 1)\end{array}$ & $\begin{array}{c}26 \cdot 2 \\
(17 \cdot 0 \text { to } 41 \cdot 2)\end{array}$ & $\begin{array}{l}-22 \cdot 5 \\
(-42 \cdot 66 \text { to }-4.64)\end{array}$ & $\begin{array}{c}0.8 \\
(0.5 \text { to } 1 \cdot 3)\end{array}$ & $\begin{array}{c}0.4 \\
(0.3 \text { to } 0.7)\end{array}$ & $\begin{array}{l}-45 \cdot 4 \\
(-56 \cdot 40 \text { to }-33 \cdot 85)\end{array}$ \\
\hline $\begin{array}{l}\text { Other communicable, maternal, } \\
\text { neonatal, and nutritional diseases }\end{array}$ & $\begin{array}{c}536 \cdot 5 \\
(433 \cdot 0 \text { to } 674 \cdot 9)\end{array}$ & $\begin{array}{c}408 \cdot 4 \\
(342 \cdot 1 \text { to } 488 \cdot 3)\end{array}$ & $\begin{array}{l}-23 \cdot 8 \\
(-33 \cdot 94 \text { to }-11 \cdot 53)\end{array}$ & $\begin{array}{c}9 \cdot 4 \\
(7 \cdot 9 \text { to } 11 \cdot 4)\end{array}$ & $\begin{array}{c}5 \cdot 9 \\
(5 \cdot 0 \text { to } 7 \cdot 0)\end{array}$ & $\begin{array}{l}-37 \cdot 4 \\
(-44 \cdot 55 \text { to }-28 \cdot 28)\end{array}$ \\
\hline $\begin{array}{l}\text { Sexually transmitted diseases } \\
\text { excluding HIV }\end{array}$ & $\begin{array}{c}257 \cdot 6 \\
(154 \cdot 7 \text { to } 396 \cdot 4)\end{array}$ & $\begin{array}{c}142 \cdot 0 \\
(87 \cdot 6 \text { to } 213 \cdot 9)\end{array}$ & $\begin{array}{l}-44 \cdot 5 \\
(-55 \cdot 96 \text { to }-32 \cdot 77)\end{array}$ & $\begin{array}{c}3 \cdot 8 \\
(2 \cdot 4 \text { to } 5 \cdot 7)\end{array}$ & $\begin{array}{c}1.9 \\
(1 \cdot 2 \text { to } 2 \cdot 9)\end{array}$ & $\begin{array}{l}-48 \cdot 4 \\
(-58 \cdot 54 \text { to }-37 \cdot 36)\end{array}$ \\
\hline Syphilis & $\begin{array}{c}250 \cdot 6 \\
(147 \cdot 4 \text { to } 389 \cdot 1)\end{array}$ & $\begin{array}{c}136 \cdot 8 \\
(82 \cdot 4 \text { to } 208 \cdot 9)\end{array}$ & $\begin{array}{l}-45 \cdot 1 \\
(-56 \cdot 56 \text { to }-33 \cdot 02)\end{array}$ & $\begin{array}{c}3 \cdot 6 \\
(2 \cdot 2 \text { to } 5 \cdot 5)\end{array}$ & $\begin{array}{c}1.9 \\
(1.1 \text { to } 2 \cdot 9)\end{array}$ & $\begin{array}{l}-48 \cdot 0 \\
(-58 \cdot 61 \text { to }-36 \cdot 83)\end{array}$ \\
\hline Chlamydial infection & $\begin{array}{c}1.5 \\
(1.1 \text { to } 1 \cdot 9)\end{array}$ & $\begin{array}{c}1.1 \\
(0.9 \text { to } 1.4)\end{array}$ & $\begin{array}{l}-23.0 \\
(-42.59 \text { to } 0.03)\end{array}$ & $\begin{array}{c}0.0 \\
(0.0 \text { to } 0.0)\end{array}$ & $\begin{array}{c}0.0 \\
(0.0 \text { to } 0.0)\end{array}$ & $\begin{array}{l}-51 \cdot 1 \\
(-64 \cdot 66 \text { to }-34 \cdot 62)\end{array}$ \\
\hline Gonococcal infection & $\begin{array}{c}3 \cdot 2 \\
(2 \cdot 5 \text { to } 3 \cdot 8)\end{array}$ & $\begin{array}{c}2 \cdot 3 \\
(2 \cdot 0 \text { to } 2 \cdot 9)\end{array}$ & $\begin{array}{l}-26.6 \\
(-41.91 \text { to }-6.75)\end{array}$ & $\begin{array}{c}0.1 \\
(0.1 \text { to } 0 \cdot 1)\end{array}$ & $\begin{array}{c}0.0 \\
(0.0 \text { to } 0.0)\end{array}$ & $\begin{array}{l}-54 \cdot 0 \\
(-64 \cdot 57 \text { to }-39 \cdot 74)\end{array}$ \\
\hline Other sexually transmitted diseases & $\begin{array}{c}2 \cdot 4 \\
(1.9 \text { to } 2 \cdot 8)\end{array}$ & $\begin{array}{c}1 \cdot 7 \\
(1 \cdot 5 \text { to } 2 \cdot 1)\end{array}$ & $\begin{array}{l}-27 \cdot 3 \\
(-42 \cdot 08 \text { to }-10 \cdot 71)\end{array}$ & $\begin{array}{c}0.1 \\
(0.0 \text { to } 0 \cdot 1)\end{array}$ & $\begin{array}{c}0.0 \\
(0.0 \text { to } 0.0)\end{array}$ & $\begin{array}{l}-53 \cdot 2 \\
(-63 \cdot 49 \text { to }-40 \cdot 60)\end{array}$ \\
\hline Hepatitis & $\begin{array}{c}162 \cdot 0 \\
(152 \cdot 8 \text { to } 171 \cdot 4)\end{array}$ & $\begin{array}{c}136 \cdot 7 \\
(123 \cdot 7 \text { to } 163 \cdot 2)\end{array}$ & $\begin{array}{l}-16 \cdot 8 \\
(-25 \cdot 24 \text { to } 2 \cdot 96)\end{array}$ & $\begin{array}{c}3 \cdot 3 \\
(3 \cdot 1 \text { to } 3 \cdot 5)\end{array}$ & $\begin{array}{c}2 \cdot 0 \\
(1 \cdot 8 \text { to } 2 \cdot 4)\end{array}$ & $\begin{array}{l}-39 \cdot 6 \\
(-45 \cdot 37 \text { to }-25 \cdot 80)\end{array}$ \\
\hline Hepatitis A & $\begin{array}{c}22 \cdot 6 \\
(7 \cdot 9 \text { to } 40 \cdot 2)\end{array}$ & $\begin{array}{c}14 \cdot 9 \\
(5 \cdot 0 \text { to } 27 \cdot 7)\end{array}$ & $\begin{array}{l}-36 \cdot 3 \\
(-50 \cdot 41 \text { to }-2 \cdot 45)\end{array}$ & $\begin{array}{c}0.3 \\
(0.1 \text { to } 0 \cdot 6)\end{array}$ & $\begin{array}{c}0.2 \\
(0.1 \text { to } 0.4)\end{array}$ & $\begin{array}{l}-41 \cdot 9 \\
(-54 \cdot 39 \text { to }-12 \cdot 04)\end{array}$ \\
\hline Hepatitis B & $\begin{array}{c}85 \cdot 0 \\
(65 \cdot 1 \text { to } 104 \cdot 0)\end{array}$ & $\begin{array}{c}68 \cdot 6 \\
(52 \cdot 0 \text { to } 86 \cdot 6)\end{array}$ & $\begin{array}{l}-19.7 \\
(-28.94 \text { to }-4.23)\end{array}$ & $\begin{array}{c}1 \cdot 9 \\
(1 \cdot 5 \text { to } 2 \cdot 2)\end{array}$ & $\begin{array}{c}1.1 \\
(0.8 \text { to } 1 \cdot 3)\end{array}$ & $\begin{array}{l}-44 \cdot 6 \\
(-50 \cdot 38 \text { to }-35 \cdot 37)\end{array}$ \\
\hline Hepatitis C & $\begin{array}{c}2 \cdot 3 \\
(0 \cdot 5 \text { to } 5 \cdot 3)\end{array}$ & $\begin{array}{c}3 \cdot 5 \\
(0.7 \text { to } 8 \cdot 2)\end{array}$ & $\begin{array}{c}51 \cdot 0 \\
(24 \cdot 40 \text { to } 86 \cdot 56)\end{array}$ & $\begin{array}{c}0.1 \\
(0.0 \text { to } 0 \cdot 1)\end{array}$ & $\begin{array}{c}0.1 \\
(0.0 \text { to } 0.1)\end{array}$ & $\begin{array}{l}-4 \cdot 2 \\
(-20 \cdot 86 \text { to } 17 \cdot 67)\end{array}$ \\
\hline Hepatitis E & $\begin{array}{c}52 \cdot 1 \\
(39 \cdot 0 \text { to } 67 \cdot 2)\end{array}$ & $\begin{array}{c}49 \cdot 7 \\
(36 \cdot 1 \text { to } 67 \cdot 5)\end{array}$ & $\begin{array}{l}-6 \cdot 1 \\
(-17.83 \text { to } 20 \cdot 41)\end{array}$ & $\begin{array}{c}1 \cdot 0 \\
(0 \cdot 7 \text { to } 1 \cdot 3)\end{array}$ & $\begin{array}{c}0.7 \\
(0.5 \text { to } 1.0)\end{array}$ & $\begin{array}{l}-30 \cdot 8 \\
(-39 \cdot 35 \text { to }-11 \cdot 37)\end{array}$ \\
\hline Other infectious diseases & $\begin{array}{c}116 \cdot 9 \\
(94 \cdot 0 \text { to } 144 \cdot 3)\end{array}$ & $\begin{array}{c}129 \cdot 8 \\
(89.6 \text { to } 164 \cdot 9)\end{array}$ & $\begin{array}{c}11.3 \\
(-21.67 \text { to } 45 \cdot 00)\end{array}$ & $\begin{array}{c}2 \cdot 4 \\
(1.9 \text { to } 3 \cdot 0)\end{array}$ & $\begin{array}{c}2.0 \\
(1.4 \text { to } 2 \cdot 5)\end{array}$ & $\begin{array}{l}-17 \cdot 7 \\
(-42 \cdot 92 \text { to } 7 \cdot 31)\end{array}$ \\
\hline Non-communicable diseases & $\begin{array}{l}26993.5 \\
(26298 \cdot 1 \text { to } \\
27639 \cdot 1)\end{array}$ & $\begin{array}{l}38267 \cdot 2 \\
(37202 \cdot 2 \text { to } 39417 \cdot 6)\end{array}$ & $\begin{array}{l}41 \cdot 7 \\
(36 \cdot 86 \text { to } 47 \cdot 00)\end{array}$ & $\begin{array}{l}782 \cdot 5 \\
(765 \cdot 5 \text { to } 798 \cdot 2)\end{array}$ & $\begin{array}{l}637 \cdot 5 \\
(620 \cdot 4 \text { to } 655 \cdot 7)\end{array}$ & $\begin{array}{l}-18 \cdot 6 \\
(-21.08 \text { to }-15 \cdot 78)\end{array}$ \\
\hline Neoplasms & $\begin{array}{l}5659 \cdot 7 \\
(5440 \cdot 4 \text { to } 5826 \cdot 6)\end{array}$ & $\begin{array}{c}8235 \cdot 7 \\
(7941.4 \text { to } 8538.9)\end{array}$ & $\begin{array}{l}45 \cdot 6 \\
\text { (39.82 to } 51 \cdot 55)\end{array}$ & $\begin{array}{l}157 \cdot 0 \\
(151 \cdot 3 \text { to } 161 \cdot 5)\end{array}$ & $\begin{array}{l}133.8 \\
(128.9 \text { to } 138.6)\end{array}$ & $\begin{array}{l}-14 \cdot 7 \\
(-17 \cdot 91 \text { to }-11 \cdot 49)\end{array}$ \\
\hline Oesophageal cancer & $\begin{array}{c}313 \cdot 1 \\
(275 \cdot 0 \text { to } 351 \cdot 5)\end{array}$ & $\begin{array}{c}440 \cdot 2 \\
(389 \cdot 2 \text { to } 516 \cdot 8)\end{array}$ & $\begin{array}{c}39.9 \\
(26 \cdot 42 \text { to } 56 \cdot 36)\end{array}$ & $\begin{array}{c}8.8 \\
(7 \cdot 8 \text { to } 9 \cdot 9)\end{array}$ & $\begin{array}{c}7 \cdot 2 \\
(6 \cdot 3 \text { to } 8 \cdot 4)\end{array}$ & $\begin{array}{l}-19 \cdot 3 \\
(-27 \cdot 13 \text { to }-9 \cdot 94)\end{array}$ \\
\hline Stomach cancer & $\begin{array}{c}763 \cdot 4 \\
(725 \cdot 6 \text { to } 803 \cdot 2)\end{array}$ & $\begin{array}{c}841 \cdot 0 \\
\text { (791.6 to } 894 \cdot 1)\end{array}$ & $\begin{array}{c}10 \cdot 1 \\
(3 \cdot 94 \text { to } 17 \cdot 43)\end{array}$ & $\begin{array}{c}21 \cdot 7 \\
(20 \cdot 6 \text { to } 22 \cdot 9)\end{array}$ & $\begin{array}{c}13 \cdot 8 \\
(13 \cdot 0 \text { to } 14 \cdot 7)\end{array}$ & $\begin{array}{l}-36 \cdot 3 \\
(-39 \cdot 82 \text { to }-32 \cdot 14)\end{array}$ \\
\hline & & & & & & 2 continues on next page) \\
\hline
\end{tabular}




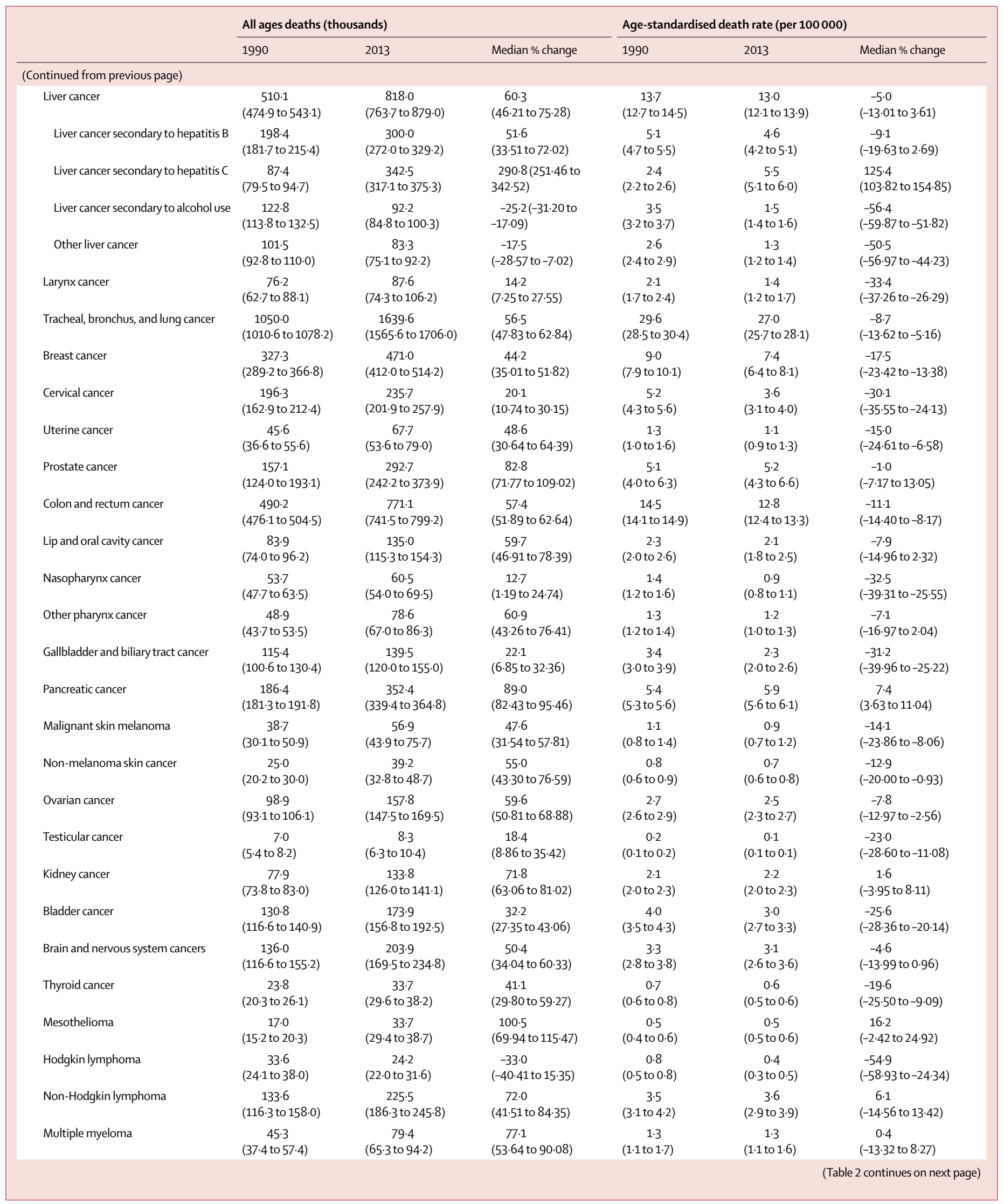




\begin{tabular}{|c|c|c|c|c|c|c|}
\hline & \multicolumn{3}{|c|}{ All ages deaths (thousands) } & \multicolumn{3}{|c|}{ Age-standardised death rate (per 100000 ) } \\
\hline & 1990 & 2013 & Median \% change & 1990 & 2013 & Median \% change \\
\hline \multicolumn{7}{|l|}{ (Continued from previous page) } \\
\hline Leukaemia & $\begin{array}{c}223 \cdot 8 \\
(215 \cdot 1 \text { to } 234 \cdot 2)\end{array}$ & $\begin{array}{c}265 \cdot 1 \\
(253 \cdot 9 \text { to } 275 \cdot 8)\end{array}$ & $\begin{array}{l}18 \cdot 7 \\
(11 \cdot 69 \text { to } 24 \cdot 19)\end{array}$ & $\begin{array}{c}5 \cdot 2 \\
(5 \cdot 0 \text { to } 5 \cdot 4)\end{array}$ & $\begin{array}{c}4 \cdot 1 \\
(3 \cdot 9 \text { to } 4 \cdot 3)\end{array}$ & $\begin{array}{l}-20 \cdot 0 \\
(-24 \cdot 12 \text { to }-16 \cdot 68)\end{array}$ \\
\hline Other neoplasms & $\begin{array}{c}250 \cdot 6 \\
(231 \cdot 8 \text { to } 305 \cdot 2)\end{array}$ & $\begin{array}{c}369 \cdot 6 \\
(328 \cdot 0 \text { to } 400 \cdot 1)\end{array}$ & $\begin{array}{c}51 \cdot 3 \\
(15 \cdot 57 \text { to } 62 \cdot 10)\end{array}$ & $\begin{array}{c}6 \cdot 2 \\
(5 \cdot 8 \text { to } 7 \cdot 3)\end{array}$ & $\begin{array}{c}5 \cdot 8 \\
(5 \cdot 2 \text { to } 6 \cdot 3)\end{array}$ & $\begin{array}{l}-4 \cdot 2 \\
(-23 \cdot 44 \text { to } 3 \cdot 32)\end{array}$ \\
\hline Cardiovascular diseases & $\begin{array}{l}12279.6 \\
(11776.6 \text { to } 12764 \cdot 1)\end{array}$ & $\begin{array}{l}17297 \cdot 5 \\
(16520 \cdot 2 \text { to } 18071 \cdot 9)\end{array}$ & $\begin{array}{l}40 \cdot 8 \\
(36 \cdot 17 \text { to } 46 \cdot 36)\end{array}$ & $\begin{array}{l}375 \cdot 5 \\
(360 \cdot 5 \text { to } 389.1)\end{array}$ & $\begin{array}{l}293.2 \\
(280 \cdot 4 \text { to } 306 \cdot 1)\end{array}$ & $\begin{array}{l}-22 \cdot 0 \\
(-24 \cdot 50 \text { to }-19 \cdot 07)\end{array}$ \\
\hline Rheumatic heart disease & $\begin{array}{c}373 \cdot 5 \\
(302 \cdot 5 \text { to } 464 \cdot 6)\end{array}$ & $\begin{array}{c}275 \cdot 1 \\
(222 \cdot 6 \text { to } 353 \cdot 9)\end{array}$ & $\begin{array}{l}-26 \cdot 5 \\
(-33 \cdot 64 \text { to }-17 \cdot 20)\end{array}$ & $\begin{array}{c}9 \cdot 8 \\
(7 \cdot 9 \text { to } 12 \cdot 2)\end{array}$ & $\begin{array}{c}4 \cdot 4 \\
(3 \cdot 5 \text { to } 5 \cdot 6)\end{array}$ & $\begin{array}{l}-55 \cdot 4 \\
(-59 \cdot 47 \text { to }-50 \cdot 11)\end{array}$ \\
\hline Ischaemic heart disease & $\begin{array}{c}5737 \cdot 5 \\
(5254 \cdot 9 \text { to } 6148 \cdot 6)\end{array}$ & $\begin{array}{c}8139.9 \\
(7322 \cdot 9 \text { to } 8758 \cdot 5)\end{array}$ & $\begin{array}{c}41 \cdot 7 \\
\text { (35.96 to } 48 \cdot 44)\end{array}$ & $\begin{array}{l}177 \cdot 3 \\
(161 \cdot 8 \text { to } 190 \cdot 2)\end{array}$ & $\begin{array}{l}137 \cdot 8 \\
(123 \cdot 9 \text { to } 148 \cdot 2)\end{array}$ & $\begin{array}{l}-22 \cdot 3 \\
(-25 \cdot 48 \text { to }-18 \cdot 68)\end{array}$ \\
\hline Cerebrovascular disease & $\begin{array}{c}4584 \cdot 8 \\
(4162 \cdot 1 \text { to } 4968 \cdot 1)\end{array}$ & $\begin{array}{c}6446 \cdot 9 \\
(5963 \cdot 0 \text { to } 7155 \cdot 2)\end{array}$ & $\begin{array}{l}40 \cdot 2 \\
(34 \cdot 43 \text { to } 49 \cdot 56)\end{array}$ & $\begin{array}{l}141 \cdot 6 \\
(128 \cdot 5 \text { to } 153 \cdot 9)\end{array}$ & $\begin{array}{l}110 \cdot 1 \\
(101 \cdot 8 \text { to } 122 \cdot 2)\end{array}$ & $\begin{array}{l}-22 \cdot 5 \\
(-25 \cdot 56 \text { to }-17 \cdot 30)\end{array}$ \\
\hline Ischaemic stroke & $\begin{array}{c}2182 \cdot 9 \\
(1923 \cdot 3 \text { to } 2430 \cdot 9)\end{array}$ & $\begin{array}{c}3272 \cdot 9 \\
(2812 \cdot 7 \text { to } 3592 \cdot 6)\end{array}$ & $\begin{array}{l}50 \cdot 2 \\
(41 \cdot 02 \text { to } 59 \cdot 27)\end{array}$ & $\begin{array}{c}71 \cdot 3 \\
(63 \cdot 0 \text { to } 79 \cdot 3)\end{array}$ & $\begin{array}{c}57 \cdot 3 \\
(49 \cdot 3 \text { to } 62 \cdot 9)\end{array}$ & $\begin{array}{l}-19.6 \\
(-24.52 \text { to }-14.97)\end{array}$ \\
\hline Haemorrhagic stroke & $\begin{array}{c}2401 \cdot 9 \\
(2109 \cdot 4 \text { to } 2669 \cdot 1)\end{array}$ & $\begin{array}{c}3174 \cdot 0 \\
(2885 \cdot 7 \text { to } 3719 \cdot 7)\end{array}$ & $\begin{array}{c}30 \cdot 7 \\
(22.23 \text { to } 49 \cdot 07)\end{array}$ & $\begin{array}{c}70 \cdot 3 \\
(61 \cdot 2 \text { to } 77 \cdot 9)\end{array}$ & $\begin{array}{c}52 \cdot 8 \\
(48 \cdot 0 \text { to } 62 \cdot 3)\end{array}$ & $\begin{array}{l}-25 \cdot 9 \\
(-30 \cdot 64 \text { to }-14.73)\end{array}$ \\
\hline Hypertensive heart disease & $\begin{array}{c}622 \cdot 1 \\
(525 \cdot 7 \text { to } 783 \cdot 9)\end{array}$ & $\begin{array}{c}1068 \cdot 6 \\
(849 \cdot 8 \text { to } 1242 \cdot 2)\end{array}$ & $\begin{array}{c}74 \cdot 1 \\
(47 \cdot 34 \text { to } 93 \cdot 73)\end{array}$ & $\begin{array}{c}19 \cdot 3 \\
(16 \cdot 4 \text { to } 24 \cdot 4)\end{array}$ & $\begin{array}{c}18 \cdot 2 \\
(14 \cdot 5 \text { to } 21 \cdot 3)\end{array}$ & $\begin{array}{l}-4.5 \\
(-18.86 \text { to } 6 \cdot 41)\end{array}$ \\
\hline Cardiomyopathy and myocarditis & $\begin{array}{c}293 \cdot 9 \\
(243 \cdot 5 \text { to } 346 \cdot 3)\end{array}$ & $\begin{array}{c}443 \cdot 3 \\
(370 \cdot 1 \text { to } 512 \cdot 0)\end{array}$ & $\begin{array}{l}51 \cdot 4 \\
(37 \cdot 27 \text { to } 61 \cdot 45)\end{array}$ & $\begin{array}{c}8 \cdot 2 \\
(6 \cdot 9 \text { to } 9.6)\end{array}$ & $\begin{array}{c}7 \cdot 1 \\
(6 \cdot 0 \text { to } 8 \cdot 3)\end{array}$ & $\begin{array}{l}-12.6 \\
(-19.98 \text { to }-7.68)\end{array}$ \\
\hline Atrial fibrillation and flutter & $\begin{array}{c}28 \cdot 9 \\
(26 \cdot 0 \text { to } 32 \cdot 4)\end{array}$ & $\begin{array}{c}112 \cdot 2 \\
\text { (97.7 to } 126 \cdot 7 \text { ) }\end{array}$ & $\begin{array}{l}288 \cdot 1 \\
(246 \cdot 32 \text { to } 335.03)\end{array}$ & $\begin{array}{c}1 \cdot 0 \\
(0 \cdot 9 \text { to } 1 \cdot 1)\end{array}$ & $\begin{array}{c}2 \cdot 0 \\
(1 \cdot 8 \text { to } 2 \cdot 3)\end{array}$ & $\begin{array}{l}100 \cdot 0 \\
(77 \cdot 55 \text { to } 124 \cdot 90)\end{array}$ \\
\hline Aortic aneurysm & $\begin{array}{c}99 \cdot 6 \\
(82 \cdot 4 \text { to } 118 \cdot 5)\end{array}$ & $\begin{array}{c}151 \cdot 5 \\
(124 \cdot 2 \text { to } 180 \cdot 0)\end{array}$ & $\begin{array}{c}52 \cdot 1 \\
(43 \cdot 75 \text { to } 60 \cdot 91)\end{array}$ & $\begin{array}{c}3 \cdot 0 \\
(2 \cdot 5 \text { to } 3 \cdot 6)\end{array}$ & $\begin{array}{c}2 \cdot 6 \\
(2 \cdot 1 \text { to } 3 \cdot 1)\end{array}$ & $\begin{array}{l}-15 \cdot 3 \\
(-20 \cdot 06 \text { to }-10 \cdot 50)\end{array}$ \\
\hline Peripheral vascular disease & $\begin{array}{c}15 \cdot 9 \\
(14 \cdot 4 \text { to } 17 \cdot 5)\end{array}$ & $\begin{array}{c}40 \cdot 5 \\
(35 \cdot 5 \text { to } 44 \cdot 9)\end{array}$ & $\begin{array}{l}155 \cdot 3 \\
\text { (126.51 to } 178 \cdot 39)\end{array}$ & $\begin{array}{c}0.5 \\
(0.5 \text { to } 0.6)\end{array}$ & $\begin{array}{c}0.7 \\
(0.6 \text { to } 0.8)\end{array}$ & $\begin{array}{l}34 \cdot 1 \\
\text { (18.77 to } 46 \cdot 62)\end{array}$ \\
\hline Endocarditis & $\begin{array}{c}45 \cdot 1 \\
(35 \cdot 6 \text { to } 58 \cdot 6)\end{array}$ & $\begin{array}{c}65 \cdot 0 \\
(48.6 \text { to } 79 \cdot 4)\end{array}$ & $\begin{array}{c}46 \cdot 3 \\
(23.88 \text { to } 65 \cdot 52)\end{array}$ & $\begin{array}{c}1 \cdot 2 \\
(1.0 \text { to } 1 \cdot 6)\end{array}$ & $\begin{array}{c}1 \cdot 0 \\
(0.8 \text { to } 1 \cdot 3)\end{array}$ & $\begin{array}{l}-12 \cdot 7 \\
(-25 \cdot 81 \text { to }-2 \cdot 80)\end{array}$ \\
\hline $\begin{array}{l}\text { Other cardiovascular and circulatory } \\
\text { diseases }\end{array}$ & $\begin{array}{c}478 \cdot 3 \\
(403 \cdot 9 \text { to } 546 \cdot 4)\end{array}$ & $\begin{array}{c}554 \cdot 6 \\
(499 \cdot 1 \text { to } 654 \cdot 2)\end{array}$ & $\begin{array}{c}15 \cdot 2 \\
(9 \cdot 38 \text { to } 32 \cdot 52)\end{array}$ & $\begin{array}{c}13 \cdot 6 \\
(11 \cdot 5 \text { to } 15 \cdot 5)\end{array}$ & $\begin{array}{c}9 \cdot 3 \\
(8 \cdot 3 \text { to } 10 \cdot 8)\end{array}$ & $\begin{array}{l}-32 \cdot 2 \\
(-35 \cdot 44 \text { to }-22 \cdot 40)\end{array}$ \\
\hline Chronic respiratory diseases & $\begin{array}{l}3490 \cdot 2 \\
(3280 \cdot 4 \text { to } 3795 \cdot 3)\end{array}$ & $\begin{array}{l}4267 \cdot 5 \\
(3996 \cdot 3 \text { to } 4694 \cdot 2)\end{array}$ & $\begin{array}{l}21.9 \\
(14.95 \text { to } 31.48)\end{array}$ & $\begin{array}{l}104 \cdot 5 \\
(98 \cdot 5 \text { to } 113 \cdot 3)\end{array}$ & $\begin{array}{l}73 \cdot 0 \\
(68 \cdot 4 \text { to } 80 \cdot 2)\end{array}$ & $\begin{array}{l}-30 \cdot 4 \\
(-34 \cdot 19 \text { to }-25 \cdot 05)\end{array}$ \\
\hline Chronic obstructive pulmonary disease & $\begin{array}{c}2421 \cdot 3 \\
(2151 \cdot 3 \text { to } 2632 \cdot 4)\end{array}$ & $\begin{array}{c}2931 \cdot 2 \\
(2626 \cdot 3 \text { to } 3215 \cdot 8)\end{array}$ & $\begin{array}{l}21 \cdot 0 \\
(12 \cdot 70 \text { to } 31 \cdot 26)\end{array}$ & $\begin{array}{c}74 \cdot 8 \\
(66 \cdot 8 \text { to } 81 \cdot 2)\end{array}$ & $\begin{array}{c}50 \cdot 7 \\
(45 \cdot 4 \text { to } 55 \cdot 6)\end{array}$ & $\begin{array}{l}-32 \cdot 3 \\
(-36 \cdot 75 \text { to }-26 \cdot 54)\end{array}$ \\
\hline Pneumoconiosis & $\begin{array}{c}251 \cdot 2 \\
(184 \cdot 0 \text { to } 317 \cdot 8)\end{array}$ & $\begin{array}{c}259 \cdot 7 \\
(201 \cdot 7 \text { to } 331 \cdot 2)\end{array}$ & $\begin{array}{c}1 \cdot 9 \\
(-15 \cdot 21 \text { to } 40 \cdot 27)\end{array}$ & $\begin{array}{c}7 \cdot 2 \\
(5 \cdot 3 \text { to } 9 \cdot 0)\end{array}$ & $\begin{array}{c}4 \cdot 3 \\
(3 \cdot 3 \text { to } 5 \cdot 5)\end{array}$ & $\begin{array}{l}-40 \cdot 5 \\
(-50 \cdot 81 \text { to }-19 \cdot 84)\end{array}$ \\
\hline Silicosis & $\begin{array}{c}55 \cdot 4 \\
(36 \cdot 7 \text { to } 77 \cdot 1)\end{array}$ & $\begin{array}{c}46 \cdot 3 \\
(32 \cdot 1 \text { to } 64 \cdot 8)\end{array}$ & $\begin{array}{l}-16 \cdot 0 \\
(-32 \cdot 85 \text { to } 6 \cdot 14)\end{array}$ & $\begin{array}{c}1 \cdot 6 \\
(1 \cdot 0 \text { to } 2 \cdot 2)\end{array}$ & $\begin{array}{c}0.8 \\
(0.5 \text { to } 1 \cdot 1)\end{array}$ & $\begin{array}{l}-50 \cdot 7 \\
(-61 \cdot 18 \text { to }-38 \cdot 06)\end{array}$ \\
\hline Asbestosis & $\begin{array}{c}21 \cdot 0 \\
(13 \cdot 9 \text { to } 30 \cdot 4)\end{array}$ & $\begin{array}{c}24 \cdot 1 \\
(17 \cdot 5 \text { to } 32 \cdot 3)\end{array}$ & $\begin{array}{l}14 \cdot 2 \\
(-10 \cdot 20 \text { to } 54 \cdot 87)\end{array}$ & $\begin{array}{c}0.6 \\
(0.4 \text { to } 0.9)\end{array}$ & $\begin{array}{c}0.4 \\
(0 \cdot 3 \text { to } 0 \cdot 5)\end{array}$ & $\begin{array}{l}-32 \cdot 6 \\
(-47 \cdot 35 \text { to }-9 \cdot 61)\end{array}$ \\
\hline Coal workers pneumoconiosis & $\begin{array}{c}28 \cdot 9 \\
(18 \cdot 2 \text { to } 43 \cdot 9)\end{array}$ & $\begin{array}{c}25 \cdot 2 \\
(19 \cdot 0 \text { to } 35 \cdot 6)\end{array}$ & $\begin{array}{l}-13 \cdot 7 \\
(-30 \cdot 65 \text { to } 23 \cdot 06)\end{array}$ & $\begin{array}{c}0.8 \\
(0.5 \text { to } 1 \cdot 2)\end{array}$ & $\begin{array}{c}0.4 \\
(0.3 \text { to } 0 \cdot 6)\end{array}$ & $\begin{array}{l}-50 \cdot 2 \\
(-60 \cdot 26 \text { to }-29 \cdot 13)\end{array}$ \\
\hline Other pneumoconiosis & $\begin{array}{c}145 \cdot 9 \\
(100 \cdot 3 \text { to } 189 \cdot 2)\end{array}$ & $\begin{array}{c}164 \cdot 1 \\
(123 \cdot 1 \text { to } 213 \cdot 6)\end{array}$ & $\begin{array}{l}10 \cdot 9 \\
(-13 \cdot 48 \text { to } 61 \cdot 23)\end{array}$ & $\begin{array}{c}4 \cdot 2 \\
(2 \cdot 9 \text { to } 5 \cdot 5)\end{array}$ & $\begin{array}{c}2 \cdot 7 \\
(2 \cdot 0 \text { to } 3 \cdot 6)\end{array}$ & $\begin{array}{l}-35 \cdot 3 \\
(-50 \cdot 19 \text { to }-6 \cdot 77)\end{array}$ \\
\hline Asthma & $\begin{array}{c}504 \cdot 3 \\
(399 \cdot 7 \text { to } 731 \cdot 8)\end{array}$ & $\begin{array}{c}489 \cdot 0 \\
(397 \cdot 7 \text { to } 676 \cdot 8)\end{array}$ & $\begin{array}{l}-2 \cdot 9 \\
(-24 \cdot 58 \text { to } 19 \cdot 21)\end{array}$ & $\begin{array}{c}13 \cdot 7 \\
(10 \cdot 8 \text { to } 20 \cdot 4)\end{array}$ & $\begin{array}{c}8 \cdot 0 \\
(6 \cdot 5 \text { to } 11 \cdot 1)\end{array}$ & $\begin{array}{l}-41 \cdot 5 \\
(-55 \cdot 17 \text { to }-28 \cdot 01)\end{array}$ \\
\hline $\begin{array}{l}\text { Interstitial lung disease and pulmonary } \\
\text { sarcoidosis }\end{array}$ & $\begin{array}{c}217 \cdot 6 \\
(128 \cdot 7 \text { to } 299 \cdot 4)\end{array}$ & $\begin{array}{c}471 \cdot 5 \\
(372 \cdot 3 \text { to } 606 \cdot 8)\end{array}$ & $\begin{array}{l}114 \cdot 1 \\
(53 \cdot 25 \text { to } 214 \cdot 86)\end{array}$ & $\begin{array}{c}6.6 \\
(3.9 \text { to } 8.9)\end{array}$ & $\begin{array}{c}8 \cdot 0 \\
(6 \cdot 3 \text { to } 10 \cdot 3)\end{array}$ & $\begin{array}{l}20 \cdot 1 \\
(-11 \cdot 89 \text { to } 74 \cdot 20)\end{array}$ \\
\hline Other chronic respiratory diseases & $\begin{array}{c}95 \cdot 8 \\
(78 \cdot 3 \text { to } 113 \cdot 8)\end{array}$ & $\begin{array}{c}116 \cdot 1 \\
(99 \cdot 2 \text { to } 136 \cdot 9)\end{array}$ & $\begin{array}{l}21 \cdot 2 \\
(7 \cdot 78 \text { to } 40 \cdot 66)\end{array}$ & $\begin{array}{c}2 \cdot 2 \\
(1 \cdot 8 \text { to } 2 \cdot 7)\end{array}$ & $\begin{array}{c}1.9 \\
(1 \cdot 6 \text { to } 2 \cdot 3)\end{array}$ & $\begin{array}{l}-15 \cdot 1 \\
(-24 \cdot 22 \text { to }-3 \cdot 39)\end{array}$ \\
\hline Cirrhosis of the liver & $\begin{array}{c}838 \cdot 0 \\
(807 \cdot 0 \text { to } 866 \cdot 7)\end{array}$ & $\begin{array}{l}1221 \cdot 1 \\
(1170 \cdot 3 \text { to } 1284 \cdot 3)\end{array}$ & $\begin{array}{l}45 \cdot 6 \\
(38 \cdot 47 \text { to } 54 \cdot 52)\end{array}$ & $\begin{array}{c}21.8 \\
(20 \cdot 9 \text { to } 22 \cdot 5)\end{array}$ & $\begin{array}{c}18 \cdot 8 \\
(18 \cdot 0 \text { to } 19 \cdot 7)\end{array}$ & $\begin{array}{l}-13 \cdot 7 \\
(-17 \cdot 75 \text { to }-8 \cdot 49)\end{array}$ \\
\hline $\begin{array}{l}\text { Cirrhosis of the liver secondary to } \\
\text { hepatitis B }\end{array}$ & $\begin{array}{c}233 \cdot 9 \\
(220 \cdot 8 \text { to } 250 \cdot 0)\end{array}$ & $\begin{array}{c}317 \cdot 4 \\
(292 \cdot 3 \text { to } 344 \cdot 6)\end{array}$ & $\begin{array}{l}35 \cdot 6 \\
(22 \cdot 66 \text { to } 49 \cdot 58)\end{array}$ & $\begin{array}{c}6 \cdot 1 \\
(5 \cdot 7 \text { to } 6 \cdot 5)\end{array}$ & $\begin{array}{c}4 \cdot 9 \\
(4 \cdot 5 \text { to } 5 \cdot 3)\end{array}$ & $\begin{array}{l}-19 \cdot 3 \\
(-26 \cdot 86 \text { to }-11 \cdot 11)\end{array}$ \\
\hline $\begin{array}{l}\text { Cirrhosis of the liver secondary to } \\
\text { hepatitis } C\end{array}$ & $\begin{array}{c}213 \cdot 1 \\
(200 \cdot 4 \text { to } 226 \cdot 7)\end{array}$ & $\begin{array}{c}357 \cdot 8 \\
(334 \cdot 3 \text { to } 386 \cdot 1)\end{array}$ & $\begin{array}{l}67 \cdot 3 \\
(54 \cdot 60 \text { to } 83 \cdot 86)\end{array}$ & $\begin{array}{c}5 \cdot 7 \\
(5 \cdot 4 \text { to } 6 \cdot 0)\end{array}$ & $\begin{array}{c}5 \cdot 6 \\
(5 \cdot 2 \text { to } 6 \cdot 0)\end{array}$ & $\begin{array}{l}-2 \cdot 4 \\
(-9.58 \text { to } 7 \cdot 48)\end{array}$ \\
\hline $\begin{array}{l}\text { Cirrhosis of the liver secondary to } \\
\text { alcohol use }\end{array}$ & $\begin{array}{c}292 \cdot 2 \\
(276 \cdot 5 \text { to } 307 \cdot 1)\end{array}$ & $\begin{array}{c}383 \cdot 8 \\
(356 \cdot 2 \text { to } 414 \cdot 7)\end{array}$ & $\begin{array}{l}31 \cdot 2 \\
(20 \cdot 27 \text { to } 43 \cdot 97)\end{array}$ & $\begin{array}{c}7 \cdot 8 \\
(7 \cdot 4 \text { to } 8 \cdot 2)\end{array}$ & $\begin{array}{c}5 \cdot 9 \\
(5 \cdot 5 \text { to } 6 \cdot 4)\end{array}$ & $\begin{array}{l}-24 \cdot 1 \\
(-30 \cdot 24 \text { to }-16 \cdot 92)\end{array}$ \\
\hline & & & & & & 2 continues on next page) \\
\hline
\end{tabular}




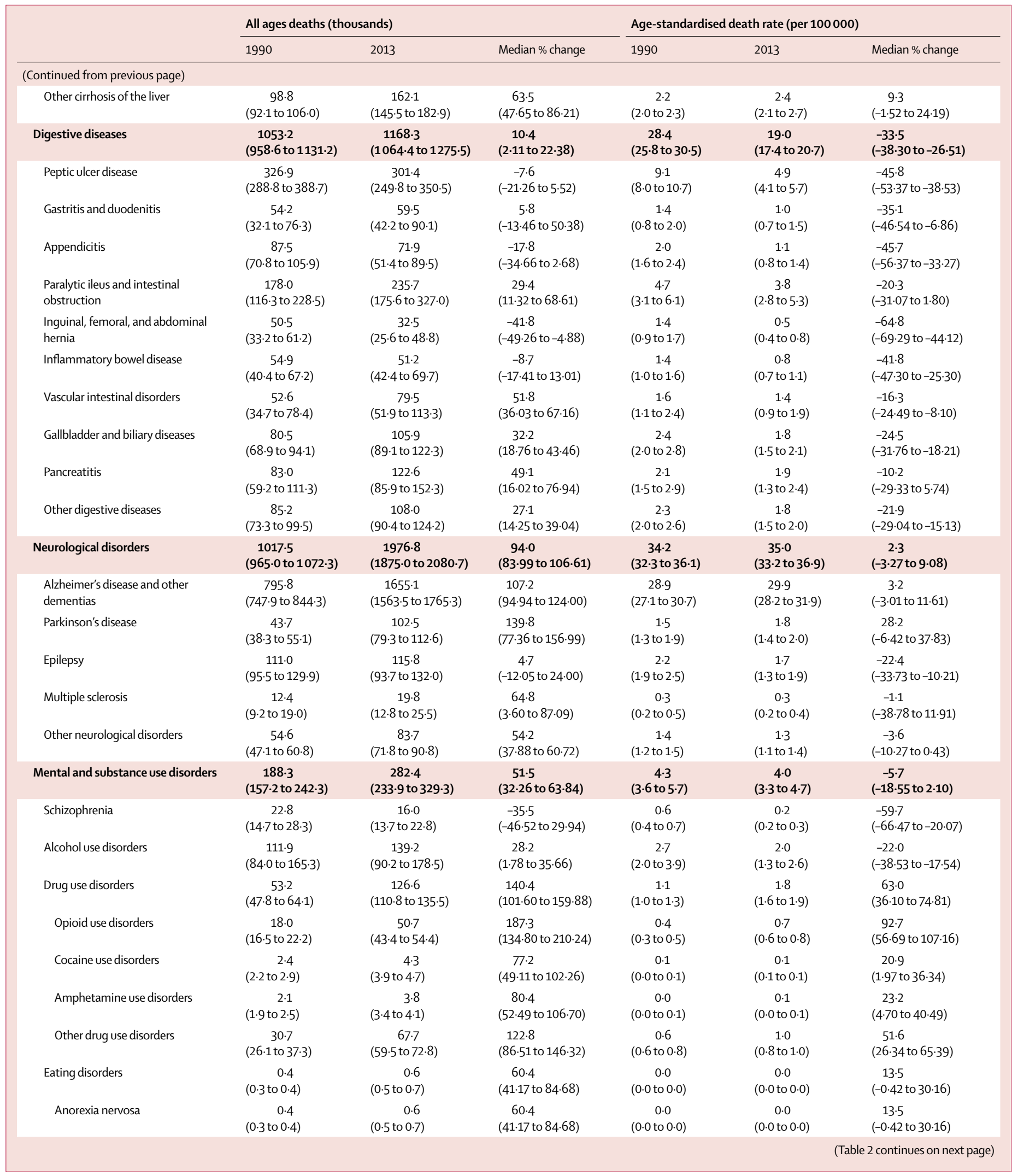




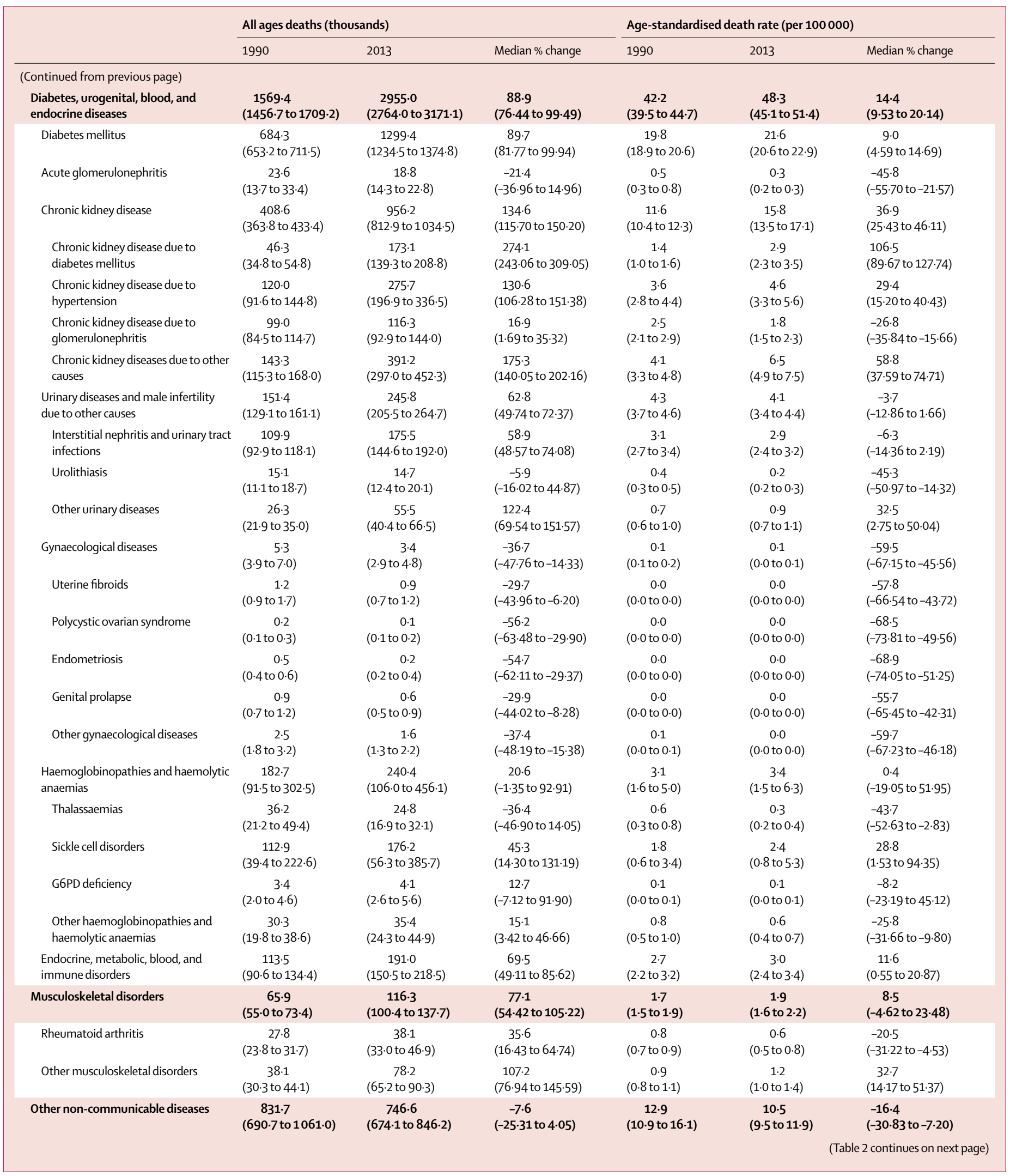




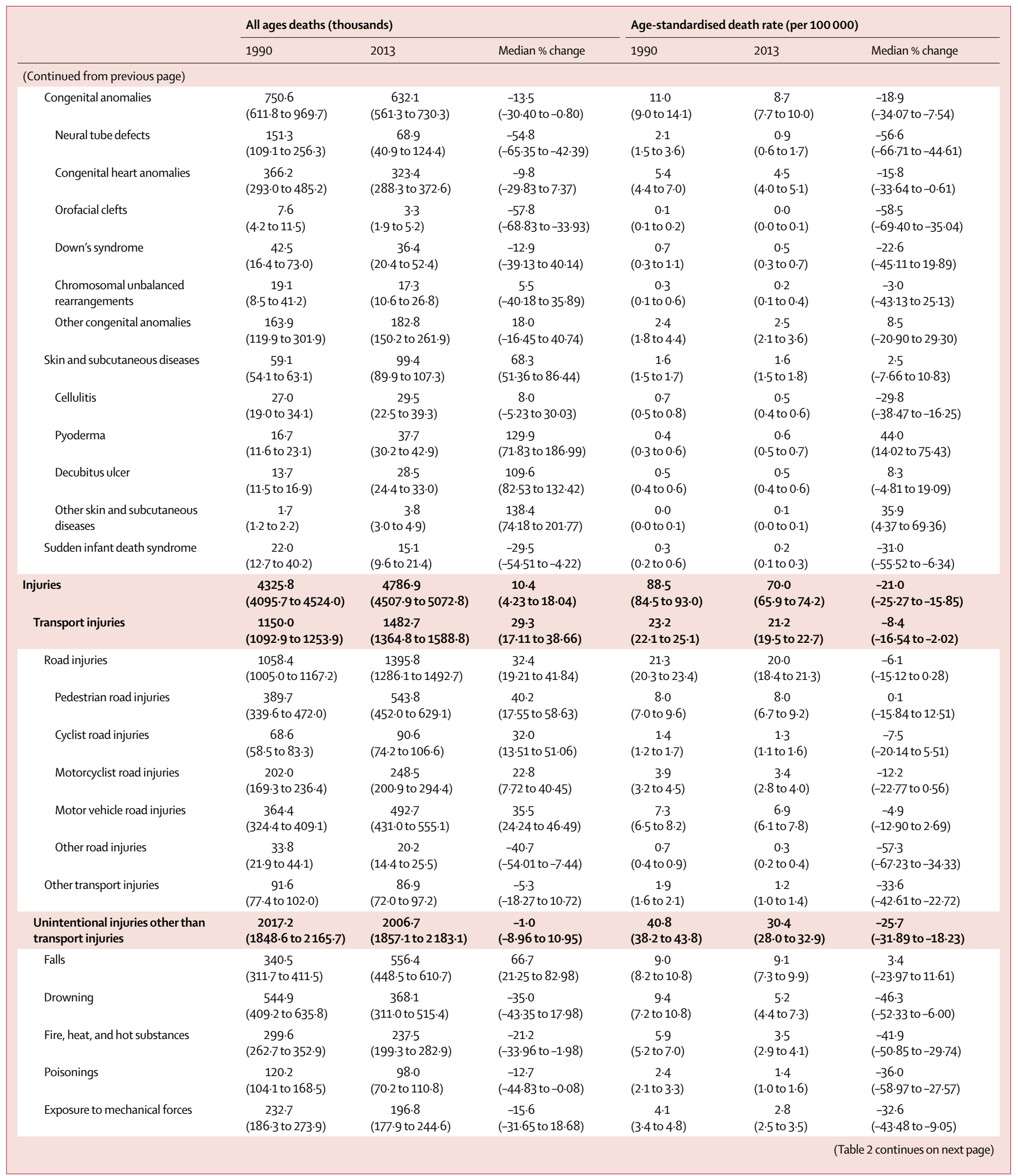




\begin{tabular}{|c|c|c|c|c|c|c|}
\hline & \multicolumn{3}{|c|}{ All ages deaths (thousands) } & \multicolumn{3}{|c|}{ Age-standardised death rate (per 100000 ) } \\
\hline & 1990 & 2013 & Median \% change & 1990 & 2013 & Median \% change \\
\hline \multicolumn{7}{|l|}{ (Continued from previous page) } \\
\hline Unintentional firearm injuries & $\begin{array}{c}51 \cdot 4 \\
(44 \cdot 5 \text { to } 60 \cdot 0)\end{array}$ & $\begin{array}{c}47 \cdot 3 \\
(41 \cdot 0 \text { to } 55 \cdot 7)\end{array}$ & $\begin{array}{l}-7 \cdot 6 \\
(-26 \cdot 30 \text { to } 13 \cdot 15)\end{array}$ & $\begin{array}{c}1 \cdot 0 \\
(0 \cdot 9 \text { to } 1 \cdot 2)\end{array}$ & $\begin{array}{c}0.7 \\
(0.6 \text { to } 0.8)\end{array}$ & $\begin{array}{l}-32.8 \\
(-46.94 \text { to }-18.02)\end{array}$ \\
\hline Unintentional suffocation & $\begin{array}{c}84 \cdot 0 \\
(26 \cdot 9 \text { to } 124 \cdot 6)\end{array}$ & $\begin{array}{c}37 \cdot 6 \\
(29 \cdot 1 \text { to } 80 \cdot 7)\end{array}$ & $\begin{array}{l}-58.3 \\
(-74.65 \text { to } 90.29)\end{array}$ & $\begin{array}{c}1 \cdot 2 \\
(0 \cdot 4 \text { to } 1 \cdot 8)\end{array}$ & $\begin{array}{c}0.5 \\
(0.4 \text { to } 1.1)\end{array}$ & $\begin{array}{l}-59 \cdot 7 \\
(-75 \cdot 13 \text { to } 71 \cdot 19)\end{array}$ \\
\hline Other exposure to mechanical forces & $\begin{array}{c}97 \cdot 2 \\
(88 \cdot 8 \text { to } 119 \cdot 9)\end{array}$ & $\begin{array}{c}111 \cdot 9 \\
(95 \cdot 5 \text { to } 122 \cdot 4)\end{array}$ & $\begin{array}{c}16.6 \\
(-13.64 \text { to } 30.93)\end{array}$ & $\begin{array}{c}1 \cdot 9 \\
(1 \cdot 7 \text { to } 2 \cdot 3)\end{array}$ & $\begin{array}{c}1.6 \\
(1.4 \text { to } 1 \cdot 7)\end{array}$ & $\begin{array}{l}-14 \cdot 7 \\
(-36 \cdot 50 \text { to }-5 \cdot 26)\end{array}$ \\
\hline Adverse effects of medical treatment & $\begin{array}{c}93 \cdot 5 \\
(77 \cdot 2 \text { to } 110 \cdot 2)\end{array}$ & $\begin{array}{c}141 \cdot 7 \\
(107 \cdot 5 \text { to } 165 \cdot 9)\end{array}$ & $\begin{array}{l}53 \cdot 2 \\
(27 \cdot 88 \text { to } 73 \cdot 38)\end{array}$ & $\begin{array}{c}2 \cdot 1 \\
(1 \cdot 8 \text { to } 2 \cdot 6)\end{array}$ & $\begin{array}{c}2 \cdot 2 \\
(1 \cdot 7 \text { to } 2 \cdot 6)\end{array}$ & $\begin{array}{c}4 \cdot 6 \\
(-11 \cdot 30 \text { to } 16 \cdot 34)\end{array}$ \\
\hline Animal contact & $\begin{array}{c}95 \cdot 5 \\
(59 \cdot 8 \text { to } 126 \cdot 6)\end{array}$ & $\begin{array}{c}79 \cdot 6 \\
(62 \cdot 3 \text { to } 138 \cdot 7)\end{array}$ & $\begin{array}{l}-22 \cdot 0 \\
(-35 \cdot 47 \text { to } 34 \cdot 57)\end{array}$ & $\begin{array}{c}1.9 \\
(1.2 \text { to } 2 \cdot 5)\end{array}$ & $\begin{array}{c}1.2 \\
(0.9 \text { to } 2 \cdot 0)\end{array}$ & $\begin{array}{l}-43 \cdot 0 \\
(-52 \cdot 23 \text { to }-2 \cdot 49)\end{array}$ \\
\hline Venomous animal contact & $\begin{array}{c}76 \cdot 3 \\
(47 \cdot 5 \text { to } 104 \cdot 7)\end{array}$ & $\begin{array}{c}57 \cdot 2 \\
(44 \cdot 1 \text { to } 102 \cdot 5)\end{array}$ & $\begin{array}{l}-30 \cdot 1 \\
(-43 \cdot 14 \text { to } 28.05)\end{array}$ & $\begin{array}{c}1.5 \\
(0.9 \text { to } 2 \cdot 0)\end{array}$ & $\begin{array}{c}0.8 \\
(0.6 \text { to } 1.5)\end{array}$ & $\begin{array}{l}-49 \cdot 3 \\
(-58 \cdot 49 \text { to }-6 \cdot 80)\end{array}$ \\
\hline Non-venomous animal contact & $\begin{array}{c}19 \cdot 2 \\
(11 \cdot 5 \text { to } 26 \cdot 3)\end{array}$ & $\begin{array}{c}22 \cdot 4 \\
(16 \cdot 4 \text { to } 36 \cdot 8)\end{array}$ & $\begin{array}{c}11 \cdot 2 \\
(-9 \cdot 33 \text { to } 73.85)\end{array}$ & $\begin{array}{c}0.4 \\
(0.2 \text { to } 0.5)\end{array}$ & $\begin{array}{c}0.3 \\
(0.2 \text { to } 0.6)\end{array}$ & $\begin{array}{l}-16 \cdot 9 \\
(-31 \cdot 27 \text { to } 25 \cdot 18)\end{array}$ \\
\hline Foreign body & $\begin{array}{c}142 \cdot 2 \\
\text { (99.5 to } 211 \cdot 6)\end{array}$ & $\begin{array}{c}165 \cdot 7 \\
(114 \cdot 8 \text { to } 219 \cdot 1)\end{array}$ & $\begin{array}{l}16 \cdot 2 \\
(-10 \cdot 22 \text { to } 45 \cdot 91)\end{array}$ & $\begin{array}{c}2 \cdot 9 \\
(2 \cdot 1 \text { to } 4 \cdot 3)\end{array}$ & $\begin{array}{c}2 \cdot 6 \\
(1 \cdot 8 \text { to } 3 \cdot 4)\end{array}$ & $\begin{array}{l}-10 \cdot 2 \\
(-29 \cdot 81 \text { to } 5 \cdot 96)\end{array}$ \\
\hline $\begin{array}{l}\text { Pulmonary aspiration and foreign } \\
\text { body in airway }\end{array}$ & $\begin{array}{c}139 \cdot 8 \\
(97 \cdot 2 \text { to } 209 \cdot 7)\end{array}$ & $\begin{array}{c}162 \cdot 1 \\
(109 \cdot 8 \text { to } 214 \cdot 7)\end{array}$ & $\begin{array}{l}15 \cdot 5 \\
(-11 \cdot 10 \text { to } 45 \cdot 78)\end{array}$ & $\begin{array}{c}2 \cdot 9 \\
(2 \cdot 0 \text { to } 4 \cdot 2)\end{array}$ & $\begin{array}{c}2.5 \\
(1.7 \text { to } 3.4)\end{array}$ & $\begin{array}{l}-10 \cdot 6 \\
(-30 \cdot 35 \text { to } 5 \cdot 87)\end{array}$ \\
\hline Foreign body in other body part & $\begin{array}{c}2 \cdot 5 \\
(1.6 \text { to } 3.5)\end{array}$ & $\begin{array}{c}3 \cdot 6 \\
(2 \cdot 7 \text { to } 5 \cdot 4)\end{array}$ & $\begin{array}{l}51 \cdot 5 \\
(-3 \cdot 11 \text { to } 84 \cdot 33)\end{array}$ & $\begin{array}{c}0.1 \\
(0 \cdot 0 \text { to } 0 \cdot 1)\end{array}$ & $\begin{array}{c}0 \cdot 1 \\
(0 \cdot 0 \text { to } 0 \cdot 1)\end{array}$ & $\begin{array}{c}6.8 \\
(-25 \cdot 59 \text { to } 31 \cdot 38)\end{array}$ \\
\hline Other unintentional injuries & $\begin{array}{c}148 \cdot 1 \\
(119 \cdot 9 \text { to } 162 \cdot 8)\end{array}$ & $\begin{array}{c}162 \cdot 8 \\
(143 \cdot 9 \text { to } 180 \cdot 3)\end{array}$ & $\begin{array}{c}9 \cdot 3 \\
(-4 \cdot 23 \text { to } 33 \cdot 11)\end{array}$ & $\begin{array}{c}3 \cdot 1 \\
(2 \cdot 5 \text { to } 3 \cdot 4)\end{array}$ & $\begin{array}{c}2 \cdot 4 \\
(2 \cdot 1 \text { to } 2 \cdot 7)\end{array}$ & $\begin{array}{l}-21 \cdot 1 \\
(-30 \cdot 53 \text { to }-4.99)\end{array}$ \\
\hline Self-harm and interpersonal violence & $\begin{array}{l}1052 \cdot 8 \\
(929 \cdot 3 \text { to } 1152 \cdot 0)\end{array}$ & $\begin{array}{c}1247 \cdot 1 \\
(1067 \cdot 2 \text { to } 1390 \cdot 9)\end{array}$ & $\begin{array}{l}18 \cdot 2 \\
(8 \cdot 38 \text { to } 29 \cdot 00)\end{array}$ & $\begin{array}{c}22 \cdot 4 \\
(19 \cdot 8 \text { to } 24 \cdot 6)\end{array}$ & $\begin{array}{c}17 \cdot 8 \\
(15 \cdot 3 \text { to } 19 \cdot 8)\end{array}$ & $\begin{array}{l}-20 \cdot 8 \\
(-27 \cdot 32 \text { to }-13 \cdot 83)\end{array}$ \\
\hline Self-harm & $\begin{array}{c}712 \cdot 0 \\
\text { (630.6 to } 784 \cdot 7)\end{array}$ & $\begin{array}{c}842 \cdot 4 \\
(718 \cdot 1 \text { to } 939 \cdot 0)\end{array}$ & $\begin{array}{l}17 \cdot 8 \\
(6 \cdot 04 \text { to } 32 \cdot 20)\end{array}$ & $\begin{array}{c}15 \cdot 8 \\
(13 \cdot 9 \text { to } 17 \cdot 3)\end{array}$ & $\begin{array}{c}12 \cdot 2 \\
(10 \cdot 4 \text { to } 13 \cdot 6)\end{array}$ & $\begin{array}{l}-23 \cdot 1 \\
(-30 \cdot 45 \text { to }-13 \cdot 97)\end{array}$ \\
\hline Interpersonal violence & $\begin{array}{c}340 \cdot 7 \\
(253 \cdot 9 \text { to } 415 \cdot 1)\end{array}$ & $\begin{array}{c}404.7 \\
(298 \cdot 7 \text { to } 496 \cdot 6)\end{array}$ & $\begin{array}{c}18 \cdot 4 \\
(10 \cdot 24 \text { to } 29 \cdot 34)\end{array}$ & $\begin{array}{c}6 \cdot 6 \\
(4 \cdot 9 \text { to } 8 \cdot 1)\end{array}$ & $\begin{array}{c}5 \cdot 6 \\
(4 \cdot 1 \text { to } 6 \cdot 9)\end{array}$ & $\begin{array}{l}-16.0 \\
(-21.65 \text { to }-8 \cdot 41)\end{array}$ \\
\hline Assault by firearm & $\begin{array}{c}127 \cdot 6 \\
(89 \cdot 9 \text { to } 165 \cdot 1)\end{array}$ & $\begin{array}{c}180 \cdot 4 \\
(120 \cdot 5 \text { to } 231 \cdot 3)\end{array}$ & $\begin{array}{c}41 \cdot 3 \\
(26 \cdot 05 \text { to } 55 \cdot 53)\end{array}$ & $\begin{array}{c}2 \cdot 5 \\
(1 \cdot 7 \text { to } 3 \cdot 2)\end{array}$ & $\begin{array}{c}2.5 \\
(1 \cdot 6 \text { to } 3 \cdot 2)\end{array}$ & $\begin{array}{l}0.0 \\
(-10 \cdot 58 \text { to } 10 \cdot 11)\end{array}$ \\
\hline Assault by sharp object & $\begin{array}{c}94 \cdot 0 \\
(65 \cdot 1 \text { to } 127 \cdot 8)\end{array}$ & $\begin{array}{c}114 \cdot 3 \\
(77 \cdot 1 \text { to } 163 \cdot 2)\end{array}$ & $\begin{array}{l}21 \cdot 0 \\
(6 \cdot 27 \text { to } 40 \cdot 68)\end{array}$ & $\begin{array}{c}1 \cdot 8 \\
(1 \cdot 3 \text { to } 2 \cdot 5)\end{array}$ & $\begin{array}{c}1 \cdot 6 \\
(1 \cdot 1 \text { to } 2 \cdot 2)\end{array}$ & $\begin{array}{l}-15 \cdot 2 \\
(-25 \cdot 63 \text { to }-1 \cdot 29)\end{array}$ \\
\hline Assault by other means & $\begin{array}{c}119 \cdot 1 \\
(84 \cdot 4 \text { to } 142 \cdot 9)\end{array}$ & $\begin{array}{c}110 \cdot 0 \\
(78 \cdot 7 \text { to } 142 \cdot 1)\end{array}$ & $\begin{array}{l}-8 \cdot 5 \\
(-16.48 \text { to } 7.97)\end{array}$ & $\begin{array}{c}2 \cdot 3 \\
(1 \cdot 6 \text { to } 2 \cdot 8)\end{array}$ & $\begin{array}{c}1.5 \\
(1 \cdot 1 \text { to } 2 \cdot 0)\end{array}$ & $\begin{array}{l}-33 \cdot 9 \\
(-39 \cdot 44 \text { to }-22 \cdot 31)\end{array}$ \\
\hline $\begin{array}{l}\text { Forces of nature, war, and legal } \\
\text { intervention }\end{array}$ & $\begin{array}{c}105 \cdot 8 \\
(77 \cdot 2 \text { to } 170 \cdot 7)\end{array}$ & $\begin{array}{c}50 \cdot 4 \\
(34 \cdot 4 \text { to } 88 \cdot 8)\end{array}$ & $\begin{array}{l}-53 \cdot 1 \\
(-58 \cdot 41 \text { to }-46 \cdot 15)\end{array}$ & $\begin{array}{c}2 \cdot 2 \\
(1 \cdot 5 \text { to } 3 \cdot 6)\end{array}$ & $\begin{array}{c}0.7 \\
(0.5 \text { to } 1 \cdot 3)\end{array}$ & $\begin{array}{l}-66 \cdot 2 \\
(-70 \cdot 18 \text { to }-61 \cdot 18)\end{array}$ \\
\hline Exposure to forces of nature & $\begin{array}{c}33 \cdot 4 \\
(19 \cdot 4 \text { to } 63 \cdot 5)\end{array}$ & $\begin{array}{c}19 \cdot 2 \\
(13 \cdot 5 \text { to } 32 \cdot 0)\end{array}$ & $\begin{array}{l}-43 \cdot 9 \\
(-58.70 \text { to }-6.92)\end{array}$ & $\begin{array}{c}0.7 \\
(0.4 \text { to } 1 \cdot 4)\end{array}$ & $\begin{array}{c}0.3 \\
(0 \cdot 2 \text { to } 0 \cdot 5)\end{array}$ & $\begin{array}{l}-60 \cdot 4 \\
(-71 \cdot 50 \text { to }-33 \cdot 98)\end{array}$ \\
\hline $\begin{array}{l}\text { Collective violence and legal } \\
\text { intervention }\end{array}$ & $\begin{array}{c}72 \cdot 4 \\
(54 \cdot 7 \text { to } 106 \cdot 5)\end{array}$ & $\begin{array}{c}31 \cdot 2 \\
(20 \cdot 3 \text { to } 57 \cdot 0)\end{array}$ & $\begin{array}{l}-57 \cdot 9 \\
(-66 \cdot 76 \text { to }-45 \cdot 85)\end{array}$ & $\begin{array}{c}1 \cdot 5 \\
(1 \cdot 1 \text { to } 2 \cdot 2)\end{array}$ & $\begin{array}{c}0.5 \\
(0.3 \text { to } 0.8)\end{array}$ & $\begin{array}{l}-69 \cdot 3 \\
(-75 \cdot 76 \text { to }-61 \cdot 23)\end{array}$ \\
\hline
\end{tabular}

while that of other haemoglobinopathies fell (table 2). The residual category of endocrine, metabolic, blood, and immune disorders increased by $11 \cdot 6 \%$ (table 3 ).

Age-standardised death rates for injuries and most specific causes of injury also fell between 1990 and 2013, but typically much less than for diseases (table 2). Age-standardised death rates for transport injury decreased since 1990, with most deaths from road injuries. The number of deaths from unintentional injuries as a whole remained essentially unchanged since 1990, although age-standardised death rates fell by more than a quarter (table 2). Especially large falls occurred for drowning, fires, unintentional suffocation, and venomous animal contact (table 2). Age-standardised death rates also fell for self-harm and interpersonal violence (table 2). Among injuries, only falls, foreign body in other body part, adverse effects of medical treatment, and pedestrian road injuries had increased age-standardised death rates, but these were not statistically significant.

For only three level 2 causes did the age-standardised death rates increase: neurological disorders; diabetes, urogenital, blood, and endocrine diseases; and musculoskeletal disorders (figure 8). Increases in the musculoskeletal disorders were driven by the category other musculoskeletal disorders; causes with high number of death in this category were systemic lupus 


\begin{tabular}{|c|c|c|c|c|c|c|}
\hline & \multicolumn{3}{|c|}{ Neonates age $<1$ month } & \multicolumn{3}{|c|}{ Children age 1-59 months } \\
\hline & 1990 (thousands) & 2013 (thousands) & Median \% change & 1990 (thousands) & 2013 (thousands) & Median \% change \\
\hline All causes & $\begin{array}{l}4506 \cdot 8 \\
(4394 \cdot 5 \text { to } \\
4612 \cdot 8)\end{array}$ & $\begin{array}{l}2614 \cdot 3 \\
(2506 \cdot 4 \text { to } \\
2723 \cdot 2)\end{array}$ & $\begin{array}{l}-42 \cdot 0 \\
(-44 \cdot 5 \text { to }-39 \cdot 5)\end{array}$ & $\begin{array}{l}7608 \cdot 5 \\
(7447 \cdot 7 \text { to } 7757 \cdot 3)\end{array}$ & $\begin{array}{l}3665 \cdot 7 \\
(3449 \cdot 4 \text { to } \\
3905 \cdot 8)\end{array}$ & $\begin{array}{l}-52 \cdot 0 \\
(-54 \cdot 7 \text { to }-48 \cdot 6)\end{array}$ \\
\hline $\begin{array}{l}\text { Communicable, maternal, } \\
\text { neonatal, and nutritional } \\
\text { diseases }\end{array}$ & $\begin{array}{l}4063.8 \\
(3935 \cdot 0 \text { to } 4181 \cdot 4)\end{array}$ & $\begin{array}{l}2275 \cdot 5 \\
(2163 \cdot 6 \text { to } \\
2374 \cdot 6)\end{array}$ & $\begin{array}{l}-44 \cdot 0 \\
(-46 \cdot 6 \text { to }-41 \cdot 4)\end{array}$ & $\begin{array}{l}6012.6 \\
(5724.0 \text { to } 6256 \cdot 5)\end{array}$ & $\begin{array}{l}2766 \cdot 2 \\
(2528 \cdot 6 \text { to } 3010 \cdot 5)\end{array}$ & $\begin{array}{l}-54 \cdot 0 \\
(-57 \cdot 5 \text { to }-50 \cdot 4)\end{array}$ \\
\hline HIV/AIDS &. &. &. & $\begin{array}{c}35 \cdot 2 \\
(32 \cdot 3 \text { to } 38 \cdot 3)\end{array}$ & $\begin{array}{c}63 \cdot 8 \\
(58 \cdot 5 \text { to } 71 \cdot 6)\end{array}$ & $\begin{array}{c}81 \cdot 1 \\
\text { (64.5 to 99.9) }\end{array}$ \\
\hline Diarrhoeal diseases & $\begin{array}{l}124 \cdot 8 \\
(110 \cdot 5 \text { to } 140 \cdot 5)\end{array}$ & $\begin{array}{c}44 \cdot 8 \\
(36 \cdot 8 \text { to } 53 \cdot 3)\end{array}$ & $\begin{array}{l}-64 \cdot 2 \\
(-70 \cdot 6 \text { to }-56 \cdot 7)\end{array}$ & $\begin{array}{l}1482 \cdot 4 \\
(1339 \cdot 7 \text { to } 1633 \cdot 3)\end{array}$ & $\begin{array}{c}474.9 \\
(398 \cdot 1 \text { to } 545 \cdot 0)\end{array}$ & $\begin{array}{l}-68.0 \\
(-72 \cdot 8 \text { to }-62.5)\end{array}$ \\
\hline Intestinal infectious diseases &.. &.. &.. & $\begin{array}{c}82 \cdot 0 \\
(46 \cdot 7 \text { to } 135 \cdot 2)\end{array}$ & $\begin{array}{c}62 \cdot 4 \\
(33 \cdot 0 \text { to } 103 \cdot 6)\end{array}$ & $\begin{array}{l}-24 \cdot 2 \\
(-37 \cdot 2 \text { to }-8 \cdot 1)\end{array}$ \\
\hline Lower respiratory infections & $\begin{array}{l}399 \cdot 3 \\
(362 \cdot 2 \text { to } 436 \cdot 9)\end{array}$ & $\begin{array}{l}196 \cdot 5 \\
(169 \cdot 3 \text { to } 224 \cdot 5)\end{array}$ & $\begin{array}{l}-50 \cdot 9 \\
(-57 \cdot 3 \text { to }-43 \cdot 7)\end{array}$ & $\begin{array}{l}1768.8 \\
\text { (1597.7 to } 1926.6)\end{array}$ & $\begin{array}{l}708 \cdot 6 \\
(628.6 \text { to } 791 \cdot 4)\end{array}$ & $\begin{array}{l}-59.9 \\
(-64.8 \text { to }-54.5)\end{array}$ \\
\hline Meningitis & $\begin{array}{c}35 \cdot 6 \\
(27 \cdot 7 \text { to } 47 \cdot 0)\end{array}$ & $\begin{array}{c}20 \cdot 6 \\
(14 \cdot 9 \text { to } 26 \cdot 8)\end{array}$ & $\begin{array}{l}-42 \cdot 6 \\
(-52 \cdot 7 \text { to }-28 \cdot 4)\end{array}$ & $\begin{array}{l}262 \cdot 5 \\
\text { (212.1 to } 346 \cdot 3)\end{array}$ & $\begin{array}{l}121 \cdot 4 \\
(90 \cdot 2 \text { to } 157 \cdot 0)\end{array}$ & $\begin{array}{l}-54 \cdot 0 \\
(-63 \cdot 4 \text { to }-41 \cdot 5)\end{array}$ \\
\hline Whooping cough &.. &. &.. & $\begin{array}{c}129 \cdot 8 \\
\text { (49.9 to } 280 \cdot 7)\end{array}$ & $\begin{array}{c}56 \cdot 4 \\
(20 \cdot 7 \text { to } 127 \cdot 0)\end{array}$ & $\begin{array}{l}-57 \cdot 1 \\
(-83 \cdot 9 \text { to } 12 \cdot 7)\end{array}$ \\
\hline Tetanus & $\begin{array}{l}216 \cdot 9 \\
(174 \cdot 8 \text { to } 370 \cdot 5)\end{array}$ & $\begin{array}{c}26 \cdot 0 \\
(12 \cdot 3 \text { to } 38 \cdot 9)\end{array}$ & $\begin{array}{l}-87 \cdot 0 \\
(-95 \cdot 8 \text { to }-80 \cdot 1)\end{array}$ & $\begin{array}{c}65 \cdot 4 \\
(48 \cdot 2 \text { to } 121 \cdot 6)\end{array}$ & $\begin{array}{c}5 \cdot 5 \\
(3 \cdot 9 \text { to } 7 \cdot 7)\end{array}$ & $\begin{array}{l}-91 \cdot 2 \\
(-95 \cdot 5 \text { to }-86 \cdot 9)\end{array}$ \\
\hline Measles &. &.. &. & $\begin{array}{l}472 \cdot 4 \\
\text { (265.3 to } 749 \cdot 9)\end{array}$ & $\begin{array}{c}82 \cdot 1 \\
(41 \cdot 7 \text { to } 145 \cdot 0)\end{array}$ & $\begin{array}{l}-83 \cdot 1 \\
(-90 \cdot 3 \text { to }-68 \cdot 5)\end{array}$ \\
\hline Malaria & $\begin{array}{c}18 \cdot 1 \\
(13 \cdot 4 \text { to } 23 \cdot 5)\end{array}$ & $\begin{array}{c}16 \cdot 8 \\
(11 \cdot 0 \text { to } 26 \cdot 4)\end{array}$ & $\begin{array}{l}-9 \cdot 6 \\
(-42 \cdot 6 \text { to } 52 \cdot 8)\end{array}$ & $\begin{array}{l}566 \cdot 3 \\
(470 \cdot 8 \text { to } 662 \cdot 0)\end{array}$ & $\begin{array}{c}570 \cdot 0 \\
(437 \cdot 5 \text { to } 733 \cdot 2)\end{array}$ & $\begin{array}{l}-1 \cdot 3 \\
(-27 \cdot 7 \text { to } 40 \cdot 9)\end{array}$ \\
\hline Preterm birth complications & $\begin{array}{l}1452 \cdot 1 \\
(1190 \cdot 6 \text { to } 1677 \cdot 3)\end{array}$ & $\begin{array}{l}693 \cdot 0 \\
(553 \cdot 6 \text { to } 853 \cdot 9)\end{array}$ & $\begin{array}{l}-52 \cdot 5 \\
(-58 \cdot 6 \text { to }-45 \cdot 0)\end{array}$ & $\begin{array}{l}118 \cdot 4 \\
\text { (80.1 to } 157 \cdot 3)\end{array}$ & $\begin{array}{c}49 \cdot 4 \\
\text { (34.5 to } 69 \cdot 8)\end{array}$ & $\begin{array}{l}-58 \cdot 3 \\
(-69 \cdot 4 \text { to }-41 \cdot 3)\end{array}$ \\
\hline $\begin{array}{l}\text { Neonatal encephalopathy } \\
\text { (birth asphyxia/trauma) }\end{array}$ & $\begin{array}{l}820 \cdot 8 \\
(651 \cdot 3 \text { to } 993 \cdot 1)\end{array}$ & $\begin{array}{l}611.5 \\
(491.9 \text { to } 724 \cdot 0)\end{array}$ & $\begin{array}{l}-25 \cdot 3 \\
(-38 \cdot 1 \text { to }-9 \cdot 8)\end{array}$ & $\begin{array}{c}53 \cdot 3 \\
(35 \cdot 5 \text { to } 72 \cdot 6)\end{array}$ & $\begin{array}{c}32 \cdot 3 \\
(21 \cdot 4 \text { to } 48 \cdot 7)\end{array}$ & $\begin{array}{l}-40 \cdot 6 \\
(-58 \cdot 2 \text { to }-9 \cdot 0)\end{array}$ \\
\hline $\begin{array}{l}\text { Neonatal sepsis and other } \\
\text { neonatal infections }\end{array}$ & $\begin{array}{l}328 \cdot 3 \\
(186 \cdot 3 \text { to } 462 \cdot 1)\end{array}$ & $\begin{array}{l}342 \cdot 2 \\
(214 \cdot 9 \text { to } 479 \cdot 3)\end{array}$ & $\begin{array}{c}4 \cdot 6 \\
(-17 \cdot 3 \text { to } 38 \cdot 5)\end{array}$ & $\begin{array}{c}18 \cdot 1 \\
(9 \cdot 2 \text { to } 28 \cdot 3)\end{array}$ & $\begin{array}{c}23 \cdot 8 \\
(13 \cdot 3 \text { to } 38 \cdot 0)\end{array}$ & $\begin{array}{l}30 \cdot 3 \\
(-15 \cdot 9 \text { to } 114 \cdot 6)\end{array}$ \\
\hline Other neonatal disorders & $\begin{array}{l}489.9 \\
(381.6 \text { to } 654 \cdot 9)\end{array}$ & $\begin{array}{l}238 \cdot 2 \\
(187 \cdot 7 \text { to } 297 \cdot 0)\end{array}$ & $\begin{array}{l}-51 \cdot 3 \\
(-62 \cdot 5 \text { to }-35 \cdot 0)\end{array}$ & $\begin{array}{c}87 \cdot 7 \\
\text { (58.5 to } 123 \cdot 5)\end{array}$ & $\begin{array}{c}38 \cdot 1 \\
(25 \cdot 0 \text { to } 59 \cdot 8)\end{array}$ & $\begin{array}{l}-57 \cdot 4 \\
(-73 \cdot 9 \text { to }-27 \cdot 8)\end{array}$ \\
\hline Nutritional deficiencies &. &.. &. & $\begin{array}{l}451 \cdot 6 \\
(376 \cdot 5 \text { to } 560 \cdot 5)\end{array}$ & $\begin{array}{l}260 \cdot 7 \\
(197 \cdot 9 \text { to } 316 \cdot 6)\end{array}$ & $\begin{array}{l}-42 \cdot 3 \\
(-52 \cdot 4 \text { to }-30 \cdot 1)\end{array}$ \\
\hline Syphilis & $\begin{array}{l}122 \cdot 6 \\
(68 \cdot 9 \text { to } 194 \cdot 3)\end{array}$ & $\begin{array}{c}63 \cdot 7 \\
(37 \cdot 0 \text { to } 98 \cdot 4)\end{array}$ & $\begin{array}{l}-47 \cdot 9 \\
(-58 \cdot 6 \text { to }-35 \cdot 5)\end{array}$ & $\begin{array}{l}100 \cdot 9 \\
(56 \cdot 9 \text { to } 157 \cdot 6)\end{array}$ & $\begin{array}{c}56 \cdot 9 \\
(32 \cdot 5 \text { to } 90 \cdot 9)\end{array}$ & $\begin{array}{l}-43 \cdot 3 \\
(-56 \cdot 8 \text { to }-28 \cdot 5)\end{array}$ \\
\hline Other communicable diseases & $\begin{array}{c}55 \cdot 3 \\
(37 \cdot 9 \text { to } 79 \cdot 1)\end{array}$ & $\begin{array}{c}22 \cdot 4 \\
(16 \cdot 4 \text { to } 30 \cdot 7)\end{array}$ & $\begin{array}{l}-59 \cdot 0 \\
(-70 \cdot 6 \text { to }-44 \cdot 7)\end{array}$ & $\begin{array}{c}317 \cdot 7 \\
(283 \cdot 8 \text { to } 355 \cdot 0)\end{array}$ & $\begin{array}{l}159 \cdot 9 \\
(134 \cdot 5 \text { to } 192 \cdot 6)\end{array}$ & $\begin{array}{l}-49 \cdot 7 \\
(-58 \cdot 7 \text { to }-39 \cdot 8)\end{array}$ \\
\hline Non-communicable diseases & $\begin{array}{l}366 \cdot 4 \\
(316 \cdot 1 \text { to } 443 \cdot 9)\end{array}$ & $\begin{array}{l}292 \cdot 3 \\
(258 \cdot 4 \text { to } 349 \cdot 7)\end{array}$ & $\begin{array}{l}-19 \cdot 8 \\
(-32 \cdot 4 \text { to } 4 \cdot 3)\end{array}$ & $\begin{array}{l}906 \cdot 4 \\
\text { (766.4 to } 1138.2)\end{array}$ & $\begin{array}{l}578 \cdot 8 \\
(462 \cdot 6 \text { to } 739 \cdot 5)\end{array}$ & $\begin{array}{l}-36 \cdot 6 \\
(-43 \cdot 4 \text { to }-23 \cdot 6)\end{array}$ \\
\hline Congenital anomalies & $\begin{array}{l}303 \cdot 6 \\
(256 \cdot 8 \text { to } 375 \cdot 4)\end{array}$ & $\begin{array}{l}246.6 \\
(219 \cdot 0 \text { to } 280 \cdot 0)\end{array}$ & $\begin{array}{l}-17 \cdot 6 \\
(-33 \cdot 1 \text { to }-0 \cdot 1)\end{array}$ & $\begin{array}{l}343 \cdot 6 \\
(255 \cdot 5 \text { to } 493 \cdot 5)\end{array}$ & $\begin{array}{l}248 \cdot 7 \\
(198.8 \text { to } 322 \cdot 9)\end{array}$ & $\begin{array}{l}-25 \cdot 7 \\
(-40 \cdot 6 \text { to }-13 \cdot 7)\end{array}$ \\
\hline $\begin{array}{l}\text { Sudden infant death } \\
\text { syndrome }\end{array}$ & $\begin{array}{c}3 \cdot 0 \\
(1.2 \text { to } 6 \cdot 1)\end{array}$ & $\begin{array}{c}2 \cdot 4 \\
(1 \cdot 1 \text { to } 4 \cdot 5)\end{array}$ & $\begin{array}{l}-13 \cdot 8 \\
(-60 \cdot 1 \text { to } 54 \cdot 0)\end{array}$ & $\begin{array}{c}19 \cdot 1 \\
(11 \cdot 2 \text { to } 34 \cdot 3)\end{array}$ & $\begin{array}{c}12 \cdot 7 \\
(8 \cdot 4 \text { to } 18 \cdot 4)\end{array}$ & $\begin{array}{l}-32 \cdot 2 \\
(-55 \cdot 5 \text { to }-4 \cdot 9)\end{array}$ \\
\hline $\begin{array}{l}\text { Other non-communicable } \\
\text { diseases }\end{array}$ & $\begin{array}{c}59 \cdot 8 \\
(52 \cdot 3 \text { to } 70 \cdot 5)\end{array}$ & $\begin{array}{c}43 \cdot 3 \\
(32 \cdot 4 \text { to } 76 \cdot 9)\end{array}$ & $\begin{array}{l}-31 \cdot 3 \\
(-43 \cdot 3 \text { to } 18 \cdot 7)\end{array}$ & $\begin{array}{l}543 \cdot 7 \\
(469 \cdot 1 \text { to } 642 \cdot 8)\end{array}$ & $\begin{array}{l}317 \cdot 4 \\
(244 \cdot 2 \text { to } 422 \cdot 8)\end{array}$ & $\begin{array}{l}-42 \cdot 8 \\
(-52 \cdot 6 \text { to }-23 \cdot 3)\end{array}$ \\
\hline Injuries & $\begin{array}{c}76 \cdot 6 \\
(58 \cdot 5 \text { to } 95 \cdot 1)\end{array}$ & $\begin{array}{c}46 \cdot 4 \\
(37 \cdot 5 \text { to } 63 \cdot 5)\end{array}$ & $\begin{array}{l}-41 \cdot 7 \\
(-53 \cdot 4 \text { to }-2 \cdot 9)\end{array}$ & $\begin{array}{l}689 \cdot 5 \\
(567 \cdot 1 \text { to } 776 \cdot 3)\end{array}$ & $\begin{array}{l}320 \cdot 7 \\
(277 \cdot 8 \text { to } 371 \cdot 5)\end{array}$ & $\begin{array}{l}-54 \cdot 6 \\
(-61 \cdot 1 \text { to }-41 \cdot 9)\end{array}$ \\
\hline Road injuries & $\begin{array}{c}4 \cdot 1 \\
(3 \cdot 3 \text { to } 5 \cdot 1)\end{array}$ & $\begin{array}{c}3 \cdot 8 \\
(2 \cdot 6 \text { to } 5 \cdot 3)\end{array}$ & $\begin{array}{l}-6 \cdot 6 \\
(-30 \cdot 8 \text { to } 30 \cdot 5)\end{array}$ & $\begin{array}{l}105 \cdot 2 \\
(88 \cdot 7 \text { to } 128 \cdot 2)\end{array}$ & $\begin{array}{c}64 \cdot 5 \\
(51 \cdot 3 \text { to } 79 \cdot 5)\end{array}$ & $\begin{array}{l}-38 \cdot 3 \\
(-52 \cdot 6 \text { to }-23 \cdot 7)\end{array}$ \\
\hline Drowning & $\begin{array}{c}2 \cdot 7 \\
(1.9 \text { to } 3.5)\end{array}$ & $\begin{array}{c}1 \cdot 8 \\
(1 \cdot 1 \text { to } 2 \cdot 8)\end{array}$ & $\begin{array}{l}-38 \cdot 2 \\
(-56 \cdot 7 \text { to } 16 \cdot 9)\end{array}$ & $\begin{array}{c}212 \cdot 3 \\
(132 \cdot 4 \text { to } 275 \cdot 1)\end{array}$ & $\begin{array}{c}80 \cdot 1 \\
(61 \cdot 7 \text { to } 111 \cdot 4)\end{array}$ & $\begin{array}{l}-63 \cdot 3 \\
(-74 \cdot 1 \text { to }-20 \cdot 4)\end{array}$ \\
\hline Other injuries & $\begin{array}{c}69 \cdot 9 \\
(52 \cdot 2 \text { to } 88 \cdot 2)\end{array}$ & $\begin{array}{c}40.8 \\
(32 \cdot 1 \text { to } 58 \cdot 0)\end{array}$ & $\begin{array}{l}-44 \cdot 1 \\
(-55 \cdot 9 \text { to }-1 \cdot 0)\end{array}$ & $\begin{array}{c}372 \cdot 0 \\
(280 \cdot 9 \text { to } 424 \cdot 3)\end{array}$ & $\begin{array}{c}176 \cdot 2 \\
\text { (153.1 to } 203 \cdot 7)\end{array}$ & $\begin{array}{l}-53 \cdot 7 \\
(-60 \cdot 8 \text { to }-37 \cdot 6)\end{array}$ \\
\hline
\end{tabular}




\begin{tabular}{|c|c|c|c|c|c|}
\hline & \multirow[t]{2}{*}{$\begin{array}{l}\text { Age-standardised } \\
\text { death rates ( } \% \text { change) }\end{array}$} & \multicolumn{2}{|c|}{$\begin{array}{l}\text { Mean absolute difference in } \\
\text { age-standardised death rate }\end{array}$} & \multicolumn{2}{|c|}{ Gini coefficient } \\
\hline & & 2013 & $\begin{array}{l}\text { Change } \\
(1990-2013)\end{array}$ & 2013 & $\begin{array}{l}\text { Change } \\
(1990-2013)\end{array}$ \\
\hline All causes & $-24 \cdot 2 \%$ & $182 \cdot 04$ & $-19 \cdot 40$ & 0.19 & 0.024 \\
\hline Communicable, maternal, neonatal, and nutritional diseases & $-40 \cdot 5 \%$ & $104 \cdot 87$ & $-42 \cdot 23$ & 0.54 & 0.068 \\
\hline HIV/AIDS and tuberculosis & $-23.0 \%$ & $35 \cdot 91$ & $-1 \cdot 37$ & 0.69 & 0.114 \\
\hline Diarrhoea, lower respiratory, and other common infectious diseases & $-49 \cdot 6 \%$ & $40 \cdot 44$ & $-29 \cdot 80$ & 0.49 & 0.027 \\
\hline Neglected tropical diseases and malaria & $-24 \cdot 5 \%$ & $9 \cdot 28$ & $-4 \cdot 37$ & 0.78 & 0.023 \\
\hline Maternal disorders & $-43 \cdot 4 \%$ & $2 \cdot 88$ & $-1 \cdot 64$ & 0.69 & 0.085 \\
\hline Neonatal disorders & $-41 \cdot 1 \%$ & $9 \cdot 75$ & $-5 \cdot 07$ & 0.44 & 0.068 \\
\hline Nutritional deficiencies & $-28.9 \%$ & $8 \cdot 00$ & $-1 \cdot 40$ & 0.59 & 0.044 \\
\hline Other communicable, maternal, neonatal, and nutritional diseases & $-37 \cdot 2 \%$ & $2 \cdot 96$ & $-1 \cdot 54$ & 0.49 & 0.011 \\
\hline Non-communicable diseases & $-18.5 \%$ & $86 \cdot 74$ & $2 \cdot 02$ & 0.13 & 0.021 \\
\hline Neoplasms & $-14 \cdot 8 \%$ & $18 \cdot 65$ & $-6 \cdot 44$ & 0.15 & -0.021 \\
\hline Cardiovascular diseases & $-21 \cdot 9 \%$ & $59 \cdot 97$ & $7 \cdot 51$ & 0.18 & 0.044 \\
\hline Chronic respiratory diseases & $-30 \cdot 1 \%$ & $32 \cdot 35$ & $-18 \cdot 44$ & 0.36 & -0.030 \\
\hline Cirrhosis & $-13 \cdot 6 \%$ & $6 \cdot 45$ & -0.08 & $0 \cdot 30$ & 0.028 \\
\hline Digestive diseases & $-33 \cdot 2 \%$ & $7 \cdot 31$ & $-3 \cdot 31$ & 0.32 & -0.001 \\
\hline Neurological disorders & $2.5 \%$ & $5 \cdot 51$ & $0 \cdot 24$ & $0 \cdot 18$ & -0.003 \\
\hline Mental and substance use disorders & $-6 \cdot 4 \%$ & 1.77 & -0.04 & 0.44 & 0.002 \\
\hline Diabetes, urogenital, blood, and endocrine diseases & $14 \cdot 5 \%$ & $16 \cdot 67$ & 3.99 & $0 \cdot 30$ & 0.026 \\
\hline Musculoskeletal disorders & $8 \cdot 3 \%$ & $0 \cdot 32$ & 0.01 & $0 \cdot 18$ & -0.012 \\
\hline Other non-communicable diseases & $-17 \cdot 6 \%$ & $2 \cdot 49$ & 0.27 & 0.24 & 0.071 \\
\hline Injuries & $-20 \cdot 8 \%$ & $17 \cdot 31$ & -0.33 & 0.23 & 0.039 \\
\hline Transport injuries & $-8 \cdot 5 \%$ & $5 \cdot 80$ & $0 \cdot 84$ & $0 \cdot 25$ & 0.048 \\
\hline Unintentional injuries & $-25 \cdot 5 \%$ & $9 \cdot 21$ & $-2 \cdot 67$ & 0.28 & 0.001 \\
\hline Self-harm and interpersonal violence & $-20 \cdot 7 \%$ & 6.06 & 0.23 & 0.34 & 0.072 \\
\hline Forces of nature, war, and legal intervention & $-65.9 \%$ & 0.85 & -1.65 & 0.90 & -0.038 \\
\hline
\end{tabular}

Figure 8: Measures of convergence for causes of death in 188 countries

erythematosus, systemic sclerosis (scleroderma), pyogenic arthritis, and chronic osteomyelitis (data not shown). The average relative difference between countries (the inter-country Gini coefficient) ranged from 0.31 for non-communicable diseases to 0.90 for forces of nature, war, and legal intervention. Mean differences in the age-standardised rates between countries ranged from 0.32 for musculoskeletal disorders to 104.87 for communicable, maternal, neonatal, and nutritional diseases. Generally, inequality was much greater for communicable, maternal, neonatal, and nutritional causes than for non-communicable causes or injuries. An important exception to that general pattern was war and disaster, which were extraordinarily unequal across countries. For neoplasms, chronic respiratory diseases, and forces of nature, war, and legal intervention the age-standardised death rate had fallen and the two convergence metrics improved significantly from 1990 to 2013. For many communicable, maternal, neonatal, and nutritional causes, age-standardised death rates and mean absolute differences decreased but relative differences increased. For digestive diseases, unintentional injuries, and other communicable, maternal, neonatal, and nutritional diseases, death rates and mean absolute differences were falling and relative difference was not significantly different than zero.

\section{Global causes of child death}

We divided child causes of death into those occurring in children younger than age 1 month and those aged 1-59 months (table 3). The number of neonatal deaths decreased from 4.5 [UI 4.4-4.6] million in 1990, to $2 \cdot 6[2 \cdot 5-2 \cdot 7]$ million in 2013, a $42 \%(40-45)$ decrease. The most important cause of neonatal death in 2013 was neonatal encephalopathy, followed by neonatal sepsis, congenital anomalies, and lower respiratory infections (table 3 ). Causes with more than a $50 \%$ reduction in the number of neonatal deaths include tetanus, diarrhoeal diseases, lower respiratory infections, other neonatal disorders, and other communicable diseases.

For children aged 1-59 months, the global number of deaths fell by $52.0 \%$ from 1990 (7.6 [UI 7.4-7.8] million) to $2013(3 \cdot 7$ [3 4-3 9 ] million). Communicable, neonatal, and nutritional causes accounted for three-quarters of deaths in 2013, the remainder from non-communicable diseases and injuries (table 3). For this age group, two causes each accounted for more than half a million deaths and collectively accounted for 
more than a third of deaths: lower respiratory infections, and malaria (table 3). Four causes accounted for 100000-500 000 deaths: diarrhoeal disease, meningitis, congenital anomalies, and nutritional deficiencies (table 3). Another seven causes each caused 50000-100000 deaths: drowning, syphilis, measles, whooping cough, intestinal infectious diseases, HIV/ AIDS, and road injuries (table 3). Deaths fell by more than 50\% between 1990, and 2013, for diarrhoeal diseases, lower respiratory infections, meningitis, whooping cough, tetanus, measles, preterm birth complications, drowning, other neonatal disorders, and other injuries. Of the causes detailed in table 3, only HIV/AIDS increased significantly from 1990, to 2013, although from 2005, to 2013, deaths fell from 25.95 (UI 24.54-27.50) per 100000 to $9.83(9 \cdot 01-11 \cdot 04)$ per 100000 . In high-income countries, cancers accounted for $5 \cdot 86 \%(5 \cdot 06-6 \cdot 66)$ of deaths for children younger than 5 years compared with only $1.02 \%(0 \cdot 89-1 \cdot 16 \%)$ in low-income countries.

Figure 9 shows death rates of children for 19 major cause groups for the $21 \mathrm{GBD}$ regions. Because infants younger than age 1 month are exposed to, at most, 1 person-month, rates in that group were high compared with children aged 1-59 months. Across regions, death rates varied widely for preterm birth complications, neonatal encephalopathy (birth asphyxia and birth trauma), other neonatal disorders and jaundice, sepsis, and lower respiratory infections (figure 9). In addition, lower respiratory infections, HIV/AIDS, congenital syphilis, malaria, diarrhoeal diseases and congenital abnormalities had an important contribution. Congenital anomalies varied by more than five-times from a high in central sub-Saharan Africa to a low in high-income Asia Pacific. Eastern, central, and western sub-Saharan Africa had substantially high death rates for children aged 1-59 months compared with other regions including south Asia (figure 9B). These higher rates were largely related to malaria, diarrhoeal diseases, measles, and nutritional disorders.

\section{Global YLLs}

Between 1990 and 2013, large falls occurred for measles, meningitis, tetanus, syphilis, and whooping cough (figure 10). Increases of $50 \%$ or more are evident for diabetes, HIV/AIDS, hypertensive heart disease, chronic kidney disease, Alzheimer's disease and other dementias, interstitial lung disease, and pancreatic cancer. Among the top ten causes in 1990, nine remain in the top ten in 2013, with HIV/AIDS moving in and tuberculosis moving to 11th. The largest percentage increases in YLLs were for HIV/AIDS $(343 \cdot 97 \%$, 95\% UI 245.48-444.17), atrial fibrillation and flutter $(211 \cdot 89 \%, 182 \cdot 55-242 \cdot 63)$, peripheral vascular disease $(119.79 \%, 101 \cdot 04-136 \cdot 78)$, and drug use disorders $(119 \cdot 22 \%, 83 \cdot 77-140 \cdot 02)$.

Identification of key transition points in the comparative importance of different causes of premature mortality will help to better inform programme evaluation. From 1990 to 2013, worldwide crude YLLs fell by about $16 \%$ (from $2005 \cdot 5$ million to $1685 \cdot 4$ million), more so for communicable, maternal, neonatal, and nutritional diseases (39\% decrease, 1098.3 million to 667.8 million) compared with non-communicable diseases (20\% increase, 674.6 million to $806 \cdot 5$ million), and injuries $(9 \%$ decrease, 232.6 million to $210 \cdot 8$ million). Recent progress with disease control programmes for HIV/AIDS and malaria is clear, as is the substantial and steady progress to prevent child deaths from neonatal disorders, diarrhoeal diseases, and lower respiratory infections; YLLs from these diseases fell by $40-65 \%$ since 1990 . The success of vaccination programmes since 1990 is also evident (figure 11), with YLLs from measles and tetanus, in particular, at very low levels in 2013. Specific trends for major noncommunicable diseases are much less evident, with incremental decreases for several leading causes of cancer, as well as from major vascular and chronic respiratory diseases, contributing to the $30 \%$ reduction in YLLs since 1990 (37021.0 to 24493.4 per 100000). With the exception of drowning, only modest progress was made in reducing premature mortality from other leading causes of injury, with the effect of the 1994 genocide in Rwanda clearly visible (figure 11).

\section{Causes of diarrhoea and lower respiratory infection}

Deaths caused by diarrhoea fell by $51 \%$ (46-56) between 1990 and 2013 (table 2). Rotavirus was the main cause of diarrhoea in children younger than 5 years. It was also the most common cause of diarrhoea deaths in this age group in 2013, with a slight decrease in the population attributable fraction since 1990, followed by cholera, Cryptosporidium, and shigellosis (table 4). At least 55.6\% of diarrhoea in 2013 was unexplained by these pathogens in all ages, an increase from $48 \cdot 1 \%$ in 1990 (table 4).

The distribution of Shigella and Aeromonas in patients had a significant ecological association with sanitation (data not shown) and along with non-typhoid Salmonella, deaths from these pathogens fell by 5.4\% (28062 deaths) since 1990. Rotavirus was the most important pathogen for children younger than age 5 years in east and southeast Asia and eastern Europe, with a population attributable fraction of 35-41\% (104-6390); although it had the lowest population attributable fraction in high-income north America, central sub-Saharan Africa, and Caribbean. Shigella was an important pathogen in north Africa and Middle East and Oceania (causing $19 \cdot 4 \%$ [11.8-29.4] of deaths, 3790 [1976-6381] deaths and $13 \cdot 9 \%[9 \cdot 5-19 \cdot 3]$ of deaths, $118 \cdot 4$ [45.4-249.6] deaths, respectively). Cryptosporidium in sub-Saharan Africa, cholera in central sub-Saharan Africa, Andean Latin America, and Oceania, and enterotoxigenic E coli were important causes of diarrhoea death. Campylobacter did not have a significant epidemiological relationship with diarrhoea in most countries and was an important cause 
only in some age groups in the GEMS data in India, Bangladesh, and Mozambique (data not shown). Clostridium difficile caused more than $45 \%$ of diarrhoea deaths in western Europe, high-income north America, high-income Asia Pacific, and Australasia in 2013, ranging from eight deaths in Australasia to 92 in highincome North America, and to a lesser extent in central Europe $(20 \cdot 1 \%[14 \cdot 0-27 \cdot 1]$ or 16 [14-19] deaths, and

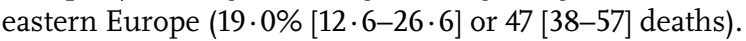

Cholera caused 45000 (23000-68000) deaths of children younger than age 5 years, with most deaths (34000 [76\%]) in central and eastern sub-Saharan Africa and south and southeast Asia. Cholera was the third leading cause of diarrhoea deaths for all ages in 2013, behind rotavirus and shigellosis (table 4). Clostridium difficile was particularly important in adults in high-income countries, where it caused as many as 95\% of diarrhoea deaths in elderly people.

Globally in 2013, pneumococcus was responsible for the largest number of lower respiratory infection deaths in children younger than age 5 years-followed by $H$ influenzae type $\mathrm{B}$, respiratory syncytial virus, and influenza-and in people of all ages (table 4). The fraction of deaths caused by lower respiratory infection in children younger than age 5 years attributable to $H$ influenzae type $\mathrm{B}$ has decreased substantially since 1990, as a result of the global scale-up of $H$ influenzae type B vaccine, with the largest decreases in high-income regions and Latin America (data not shown), where vaccine coverage is highest. The fraction of lower respiratory infection deaths in children younger than 5 years attributable to pneumococcus also fell in highincome regions such as western Europe, north America and Australasia, caused by the scale-up of pneumococcal conjugate vaccine, but continued to account for a large proportion of such deaths in eastern Europe and elsewhere. Many lower respiratory infection deaths attributable to the four pathogens occurred in older populations.

\section{Country-specific probabilities of death during childhood and young adolescence}

We computed conditional probabilities of death for three phases of life (children and young adolescents, reproductive age, and middle age) by country and cause; conditional probabilities are a useful summary because the values are readily interpretable. The probability of death in children and adolescents (age 0-14 years) varied greatly between and within regions, from a low of three per 1000 girls in Iceland to a high of 179 per 1000 boys in Guinea-Bissau (figure appendix 1). In the more demographically advanced regions (measured by mean age of death, fertility, and mortality change) the probability of death was below ten per 1000 people for both sexes in all countries except Albania, Bulgaria, Belarus, Brunei, Moldova, Macedonia, Romania, Russia, and Ukraine. Causes of death were
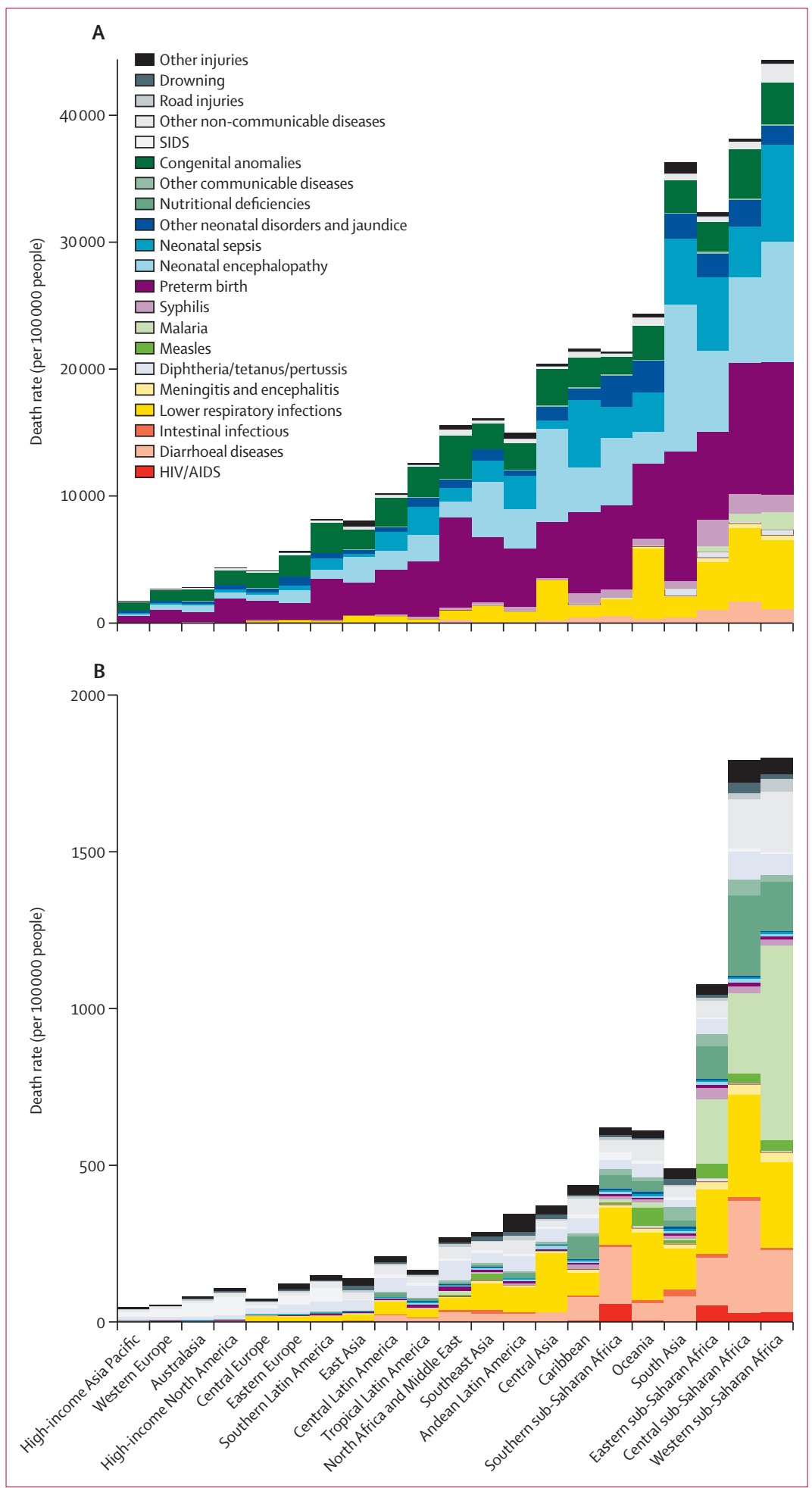

Figure 9: Child death rates by region and cause groups in 2013

(A) Of children younger than age 1 month per person-year of exposure. (B) Of children aged 1-59 months per person-year of exposure. The set of causes is mutually exclusive and collectively exhaustive. SIDS=sudden infant death syndrome. 


\begin{tabular}{|c|c|c|c|c|}
\hline \multicolumn{2}{|r|}{1990 mean rank $(95 \%$ UI) } & \multicolumn{2}{|l|}{2013 mean rank $(95 \% \mathrm{UI})$} & Median $\%$ change \\
\hline 1.0 (1 to 1$)$ & 1 Lower respiratory infections & 1 Ischaemic heart disease & $1.0(1$ to 1$)$ & $31 \%$ (24 to 41 ) \\
\hline 2.0 (2 to 2$)$ & 2 Diarrhoeal diseases & 2 Lower respiratory infections & $2 \cdot 3$ (2 to 3$)$ & $-48 \%(-54$ to -43$)$ \\
\hline 3.0 (3 to 4$)$ & 3 Preterm birth & 3 Cerebrovascular disease & $2 \cdot 7(2$ to 3$)$ & $24 \%(18$ to 32$)$ \\
\hline 4.0 (4 to 4$)$ & 4 Ischaemic heart disease & 4 Diarrhoeal diseases & $5.5(4$ to 8$)$ & $-62 \%(-66$ to -57$)$ \\
\hline $5 \cdot 1$ (5 to 6$)$ & 5 Cerebrovascular disease & 5 Road injuries & 5.9 (4 to 8$)$ & $15 \%$ (2 to 23 ) \\
\hline $6.4(5$ to 9$)$ & 6 Neonatal encephalopathy & 6 HIV/AIDS & 6.0 (4 to 8$)$ & $344 \%$ (245 to 444$)$ \\
\hline $7.5(6$ to 9$)$ & 7 Tuberculosis & 7 Preterm birth & 6.3 (4 to 9 ) & $-53 \%(-59$ to -45$)$ \\
\hline $8.0(6$ to 10$)$ & 8 Malaria & 8 Malaria & $6.9(4$ to 10$)$ & $-5 \%(-26$ to 24$)$ \\
\hline $8.9(6$ to 11$)$ & 9 Congenital anomalies & 9 Neonatal encephalopathy & $8 \cdot 7(6$ to 11$)$ & $-26 \%(-38$ to -11$)$ \\
\hline $9 \cdot 6$ (8 to 11$)$ & 10 Road injuries & 10 Congenital anomalies & $10 \cdot 3$ (8 to 12 ) & $-18 \%(-33$ to -4$)$ \\
\hline $11 \cdot 5$ (11 to 13$)$ & 11COPD & 11 Tuberculosis & $11 \cdot 1$ (10 to 12$)$ & $-31 \%(-40$ to -24$)$ \\
\hline $12 \cdot 2$ (7 to 18$)$ & 12 Measles & 12 COPD & $11 \cdot 3$ (10 to 12$)$ & $-1 \%(-9$ to 9$)$ \\
\hline $13.5(12$ to 18$)$ & 13 Drowning & 13 Cirrhosis & 13.4 (13 to 15$)$ & $36 \%(28$ to 45$)$ \\
\hline 14.5 (12 to 18$)$ & 14 Protein-energy malnutrition & 14 Self-harm & $14 \cdot 4$ (13 to 16$)$ & $9 \%(-3$ to 24$)$ \\
\hline $15 \cdot 0$ (12 to 17$)$ & 15 Meningitis & 15 Lung cancer & $15 \cdot 0$ (14 to 16$)$ & $39 \%$ (31 to 48$)$ \\
\hline 15.9 (14 to 18$)$ & 16 Self-harm & 16 Neonatal sepsis & 15.7 (12 to 22$)$ & $6 \%(-16$ to 38$)$ \\
\hline 17.1 (12 to 25$)$ & 17 Neonatal sepsis & 17 Diabetes & $17 \cdot 2$ (16 to 19$)$ & $67 \%$ (59 to 77$)$ \\
\hline 18.0 (17 to 19$)$ & 18 Cirrhosis & 18 Protein-energy malnutrition & 17.9 (16 to 22$)$ & $-28 \%(-40$ to -15$)$ \\
\hline $18.5(12$ to 21$)$ & 19 Tetanus & 19 Chronic kidney disease & 20.6 (19 to 25$)$ & $90 \%$ (74 to 103$)$ \\
\hline $19 \cdot 6$ (18 to 21$)$ & 20 Lung cancer & 20 Drowning & $20.7(16$ to 24$)$ & $-46 \%(-54$ to 3$)$ \\
\hline $21 \cdot 4$ (20 to 23$)$ & 21 Maternal disorders & 21 Liver cancer & $21 \cdot 1$ (19 to 24$)$ & $42 \%$ (26 to 58$)$ \\
\hline $22.7(16$ to 33$)$ & 22 Syphilis & 22 Interpersonal violence & $21 \cdot 2(18$ to 27$)$ & $10 \%$ (2 to 21$)$ \\
\hline 23.5 (21 to 30$)$ & 23 Interpersonal violence & 23 Meningitis & 22.9 (19 to 26$)$ & $-43 \%(-53$ to -33$)$ \\
\hline $24 \cdot 1$ (23 to 26$)$ & 24 Stomach cancer & 24 Hypertensive heart disease & $24 \cdot 5$ (20 to 29$)$ & $56 \%$ (33 to 75$)$ \\
\hline $25 \cdot 2$ (22 to 29$)$ & 25 Fire and heat & 25 Stomach cancer & $25 \cdot 0$ (23 to 27$)$ & $-2 \%(-9$ to 5$)$ \\
\hline $26.8(25$ to 29$)$ & 26 Diabetes & 26 Maternal disorders & $26 \cdot 1$ (24 to 29$)$ & $-23 \%(-32$ to -12$)$ \\
\hline 28.4 (23 to 34$)$ & 27 HIV/AIDS & 27 Colorectal cancer & $27.9(26$ to 30$)$ & $44 \%(38$ to 49$)$ \\
\hline 28.6 (22 to 33$)$ & 28 Asthma & 28 Falls & 28.8 (26 to 33$)$ & $18 \%(-14$ to 40$)$ \\
\hline 28.7 (27 to 32) & 29 Liver cancer & 29 Alzheimer disease & $29 \cdot 3(27$ to 31$)$ & $89 \%$ (81 to 103$)$ \\
\hline $31 \cdot 3$ (28 to 35$)$ & 30 Other cardiovascular & 30 Breast cancer & $31.9(30$ to 35$)$ & $37 \%$ (28 to 46$)$ \\
\hline $32.6(28$ to 36$)$ & 31 Falls & 31 Cardiomyopathy & $33 \cdot 3$ (30 to 38$)$ & $32 \%$ (14 to 47 ) \\
\hline $33 \cdot 2$ (29 to 39$)$ & 32 Rheumatic heart disease & 32 Asthma & $33 \cdot 7$ (27 to 37$)$ & $-22 \%(-35$ to -4$)$ \\
\hline $33 \cdot 4(22$ to 48$)$ & 33 Typhoid fever & 33 Other cardiovascular & $33 \cdot 7$ (30 to 37$)$ & $-12 \%(-17$ to 4$)$ \\
\hline $34 \cdot 3$ (29 to 39$)$ & 34 Hypertensive heart disease & 34 Fire and heat & 34.5 (30 to 38$)$ & $-35 \%(-46$ to -15$)$ \\
\hline $35 \cdot 6(25$ to 46$)$ & 35 Iron-deficiency anaemia & 35 Syphilis & 34.8 (25 to 46$)$ & $-46 \%(-57$ to -33$)$ \\
\hline 35.8 (33 to 40$)$ & 36 Chronic kidney disease & 36 Sickle cell & $35 \cdot 0(17$ to 63$)$ & $42 \%(8$ to 138$)$ \\
\hline $37 \cdot 2$ (19 to 61$)$ & 37 Whooping cough & 37 Typhoid fever & $35.7(24$ to 52$)$ & $-13 \%(-27$ to 1$)$ \\
\hline $37 \cdot 2$ (35 to 39$)$ & 38 Colorectal cancer & 38 Oesophageal cancer & $37 \cdot 2$ (34 to 40$)$ & $31 \%(18$ to 48$)$ \\
\hline 38.6 (36 to 41$)$ & 39 Leukaemia & 39 Leukaemia & 38.7 (37 to 41$)$ & $-9 \%(-16$ to -3$)$ \\
\hline $41.0(36$ to 45$)$ & 40 Peptic ulcer disease & 40 Interstitial lung disease & $40 \cdot 8$ (36 to 48$)$ & $86 \%$ (26 to 194$)$ \\
\hline $41 \cdot 3(38$ to 44$)$ & 41 Breast cancer & 41 Rheumatic heart disease & 41.9 (37 to 48$)$ & $-37 \%(-44$ to -26$)$ \\
\hline $41 \cdot 3$ (37 to 46$)$ & 42 Cardiomyopathy & 42 Peptic ulcer disease & $43 \cdot 8$ (40 to 51$)$ & $-20 \%(-36$ to -6$)$ \\
\hline $43 \cdot 3$ (33 to 53$)$ & 43 Pulmonary aspiration & 43 Measles & 43.8 (30 to 62$)$ & $-83 \%(-90$ to -68$)$ \\
\hline $44 \cdot 9(42$ to 48$)$ & 44 Alzheimer's disease & 44 Pancreatic cancer & $44 \cdot 2$ (42 to 48 ) & $74 \%(67$ to 80$)$ \\
\hline $45 \cdot 8(42$ to 49$)$ & 45 Oesophageal cancer & 45 Iron-deficiency anaemia & $45 \cdot 2$ (36 to 59$)$ & $-37 \%(-52$ to -21$)$ \\
\hline 46.1 (24 to 71$)$ & 46 Sickle cell & 46 Cervical cancer & $46 \cdot 2$ (42 to 54$)$ & $14 \%$ (4 to 23 ) \\
\hline 48.4 (40 to 54$)$ & \begin{tabular}{|l}
47 Poisonings \\
\end{tabular} & 47 Brain cancer & $47 \cdot 1$ (42 to 54$)$ & $27 \%(10$ to 40$)$ \\
\hline $49 \cdot 2$ (38 to 79$)$ & 48 Unintentional suffocation & 48 Pulmonary aspiration & 47.4 (39 to 59 ) & $-22 \%(-40$ to 18$)$ \\
\hline $\begin{array}{l}49 \cdot 2 \text { (44 to } 59) \\
49 \cdot 3(44 \text { to } 54)\end{array}$ & \begin{tabular}{|l}
49 Encephalitis \\
50 Epilepsy
\end{tabular} & $\begin{array}{l}49 \text { Endocrine, metabolic, blood, } \\
\text { and immune disorders }\end{array}$ & $\mid 48 \cdot 4$ (43 to 54$)$ & $29 \%$ (7 to 49 ) \\
\hline \multirow{6}{*}{$49 \cdot 3(44$ to 54$)$} & 53 Cervical cancer & 50 Lymphoma & 49.6 (45 to 55$)$ & $43 \%$ (23 to 57 ) \\
\hline & 57 Brain cancer & & & \\
\hline & $\begin{array}{l}58 \text { Endocrine, metabolic, blood, } \\
\text { and immune disorders }\end{array}$ & & & \\
\hline & 62 Lymphoma & 60 Poisonin & & \\
\hline & 64 Interstitial lung disease & 69 Tetanus & & $\begin{array}{l}\text { Group } 1 \\
\text { Non-communicable }\end{array}$ \\
\hline & 66 Pancreatic cancer & 77 Unintentional suffocation & & $\square$ Injuries \\
\hline
\end{tabular}

Figure 10: Top 50 causes of global years of life lost in 1990 and 2013

An interactive version of this figure is available at http://vizhub.healthdata.org/gbd-compare/. COPD=chronic obstructive pulmonary disease.

dominated by congenital anomalies and neonatal causes. In southeast Asia, Malaysia had the lowest probability of death and Laos the highest, with substantial mortality from lower respiratory infections, diarrhoea, and neonatal disorders. Drowning was an important cause of death for children in this region. In 


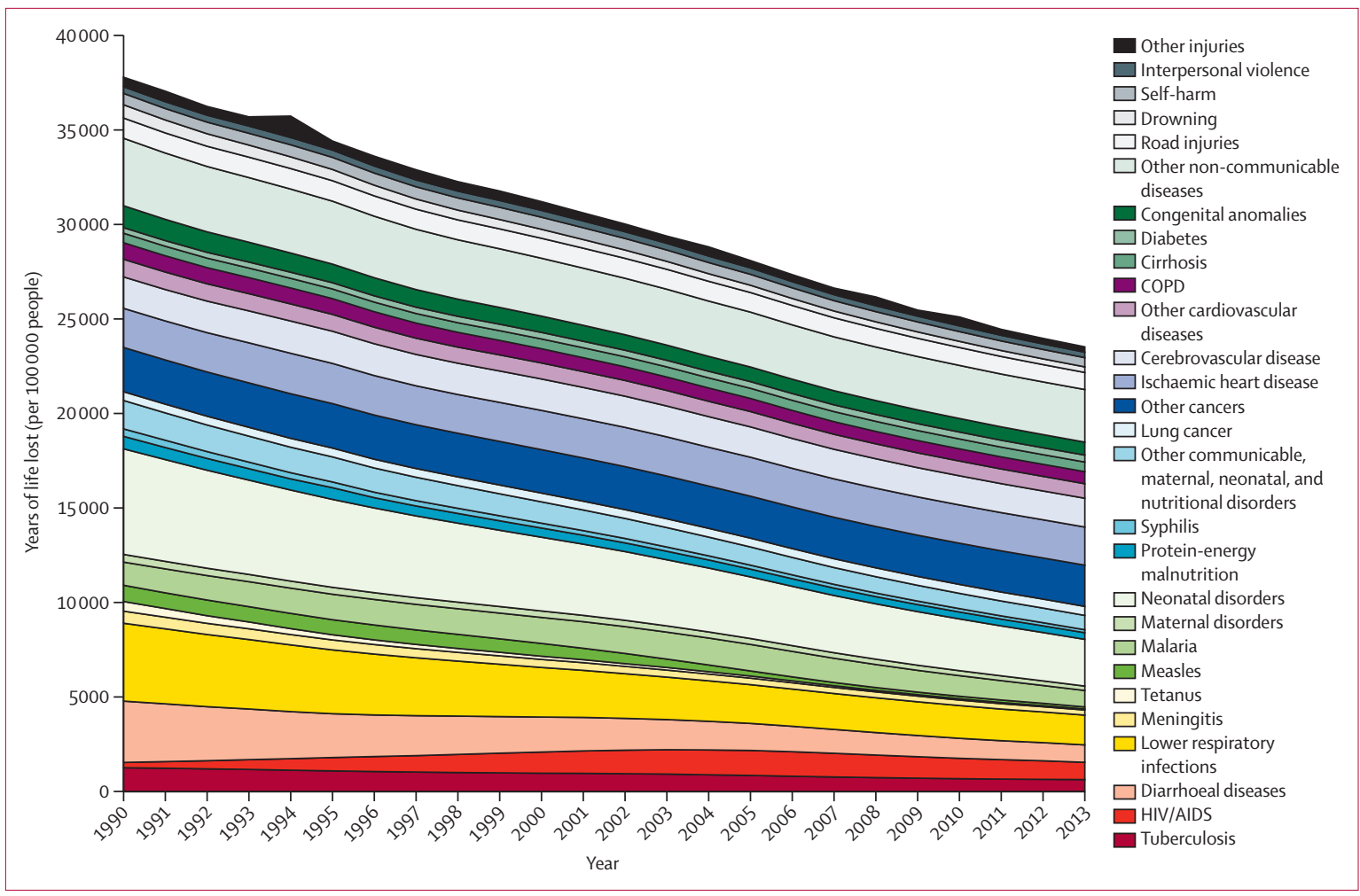

Figure 11: Global years of life lost by large cause groupings for 1990 to 2013 $\mathrm{COPD}=$ chronic obstructive pulmonary disease.

central Asia, probabilities of death ranged from 17 per 1000 people in Armenia to 65 per 1000 people in Turkmenistan, with unusually high contributions from lower respiratory infections; drowning was also an important cause. Bahrain, Oman, and United Arab Emirates had death rates below ten per 1000 for girls, whereas in Sudan and Yemen, they were more than 40 per 1000. Mortality in these countries was dominated by congenital and neonatal causes. Pulmonary aspiration and foreign body in trachea and lung was an unusually high cause of child death in Bolivia based on the available vital registration data. In south Asia, the contribution of drowning in Bangladesh was notable but mortality was still dominated by neonatal causes, diarrhoea, and pneumonia in all the countries of the region. Throughout sub-Saharan Africa, patterns of causes of death varied but malaria, HIV/AIDS, tuberculosis, nutritional deficiencies, and haemoglobinopathies played an important part in many countries. Mauritius, Seychelles, and Cape Verde all had much lower probabilities of death during childhood than did other countries in this region. Countries with the highest probabilities of death in central and western sub-Saharan Africa had important contributions from malaria. Interpersonal violence was an important contributor to the probability of death in children and adolescents in many countries of Latin America and several in southern Africa.

\section{Country-specific probabilities of death during reproductive age}

The probability of death in reproductive-age adults (exact age 15 years to exact age $50\left[{ }_{35} \mathrm{q}_{15}\right]$ ) ranged from $1.2 \%$ for women in Andorra to $52 \%$ for men in Lesotho (figure appendix 2). In high-income regions, the probability of death was generally twice as high for men as for women. Across all countries, transport injuries made an important contribution, especially in men.

Among men in low-income countries, suicide and transport accidents made important contributions. In some countries, such as Norway and USA, drug and alcohol use disorders account for more than $8 \%$ of the total probability of dying in this age interval for either sex. In central Europe, interpersonal violence was high in Albania, drug and alcohol disorders were notable in Poland, Croatia, Slovakia, and Montenegro, and cirrhosis was a common cause in Bulgaria, Croatia, Hungary, Poland, Romania, Slovakia, and Slovenia. In east Asia, liver cancer was an important cause of death. In southeast Asia, maternal mortality in Laos, Cambodia, Myanmar, and Timor-Leste were important contributors for women. For men in the same region, interpersonal violence in Philippines, Sri Lanka, and Thailand were notable; suicide in Sri Lanka also accounted for a large probability of death $(2 \cdot 2 \%)$. Other studies have suggested that interpersonal violence in Philippines is concentrated in Mindanao. ${ }^{82}$ 


\begin{tabular}{|c|c|c|c|c|c|c|c|c|c|}
\hline & \multicolumn{4}{|c|}{ Children younger than age 5 years } & \multicolumn{5}{|l|}{ All ages } \\
\hline & \multicolumn{2}{|l|}{1990} & \multicolumn{2}{|l|}{2013} & \multicolumn{2}{|l|}{1990} & \multicolumn{2}{|l|}{2013} & \multirow{2}{*}{$\begin{array}{l}\text { Rate of change } \\
\text { for deaths } \\
1990-2013(\%)\end{array}$} \\
\hline & $\begin{array}{l}\text { Deaths } \\
\text { (thousands) }\end{array}$ & $\begin{array}{l}\text { Population } \\
\text { attributable } \\
\text { fractions (\%) }\end{array}$ & $\begin{array}{l}\text { Deaths } \\
\text { (thousands) }\end{array}$ & $\begin{array}{l}\text { Population } \\
\text { attributable } \\
\text { fractions (\%) }\end{array}$ & $\begin{array}{l}\text { Deaths } \\
\text { (thousands) }\end{array}$ & $\begin{array}{l}\text { Population } \\
\text { attributable } \\
\text { fractions (\%) }\end{array}$ & $\begin{array}{l}\text { Deaths } \\
\text { (thousands) }\end{array}$ & $\begin{array}{l}\text { Population } \\
\text { attributable } \\
\text { fractions (\%) }\end{array}$ & \\
\hline \multicolumn{10}{|l|}{ Diarrhoea } \\
\hline Adenovirus & $\begin{array}{c}63 \cdot 0 \\
(44.4 \text { to 89.9) }\end{array}$ & $\begin{array}{c}3.9 \\
(2.7 \text { to } 5.5)\end{array}$ & $\begin{array}{c}18 \cdot 3 \\
\text { (11.8 to 25.5) }\end{array}$ & $\begin{array}{c}3.5 \\
\text { (2.4 to } 4.9)\end{array}$ & $\begin{array}{c}81.3 \\
(55.9 \text { to } 115.9)\end{array}$ & $\begin{array}{c}3 \cdot 2 \\
(2 \cdot 1 \text { to } 4 \cdot 4)\end{array}$ & $\begin{array}{c}31 \cdot 8 \\
(21 \cdot 5 \text { to } 44 \cdot 7)\end{array}$ & $\begin{array}{c}2.5 \\
(1.7 \text { to } 3.6)\end{array}$ & $\begin{array}{l}-61 \cdot 2 \% \\
(-67 \cdot 8 \text { to }-52 \cdot 5)\end{array}$ \\
\hline Aeromonas & $\begin{array}{c}12 \cdot 3 \\
(6 \cdot 4 \text { to } 21 \cdot 4)\end{array}$ & $\begin{array}{c}0.8 \\
(0.4 \text { to } 1.3)\end{array}$ & $\begin{array}{c}5 \cdot 5 \\
(2 \cdot 6 \text { to } 10 \cdot 1)\end{array}$ & $\begin{array}{c}1.1 \\
(0.5 \text { to } 1.9)\end{array}$ & $\begin{array}{c}28 \cdot 0 \\
(16 \cdot 7 \text { to } 44 \cdot 9)\end{array}$ & $\begin{array}{c}1.1 \\
(0.7 \text { to } 1.7)\end{array}$ & $\begin{array}{c}13 \cdot 0 \\
\text { (7.3 to } 22 \cdot 0)\end{array}$ & $\begin{array}{c}1.0 \\
(0.6 \text { to } 1.7)\end{array}$ & $\begin{array}{l}-53 \cdot 6 \% \\
(-66 \cdot 1 \text { to }-37 \cdot 8)\end{array}$ \\
\hline Amoebiasis & $\begin{array}{c}5 \cdot 8 \\
(2 \cdot 6 \text { to } 10 \cdot 0)\end{array}$ & $\begin{array}{c}0.4 \\
(0.2 \text { to } 0.6)\end{array}$ & $\begin{array}{c}1.3 \\
(0.6 \text { to } 2 \cdot 3)\end{array}$ & $\begin{array}{c}0.2 \\
\text { (0.1 to } 0.5)\end{array}$ & $\begin{array}{c}18 \cdot 3 \\
(8.9 \text { to } 30 \cdot 6)\end{array}$ & $\begin{array}{c}0.7 \\
\text { (0.3 to } 1.2)\end{array}$ & $\begin{array}{c}11 \cdot 3 \\
\text { (5.0 to } 19 \cdot 7)\end{array}$ & $\begin{array}{c}0.9 \\
(0.4 \text { to } 1.6)\end{array}$ & $\begin{array}{l}-39 \cdot 1 \% \\
(-57 \text { to }-17 \cdot 5)\end{array}$ \\
\hline Campylobacter enteritis & $\begin{array}{c}20.8 \\
\text { (11.0 to 31.9) }\end{array}$ & $\begin{array}{c}1.3 \\
(0.7 \text { to } 2 \cdot 0)\end{array}$ & $\begin{array}{c}9.5 \\
(3.7 \text { to } 15 \cdot 7)\end{array}$ & $\begin{array}{c}1.8 \\
(0.7 \text { to } 3.0)\end{array}$ & $\begin{array}{c}28 \cdot 4 \\
(16 \cdot 4 \text { to } 42 \cdot 8)\end{array}$ & $\begin{array}{c}1.1 \\
(0.6 \text { to } 1.6)\end{array}$ & $\begin{array}{c}14 \cdot 1 \\
(6 \cdot 9 \text { to } 22 \cdot 4)\end{array}$ & $\begin{array}{c}1.1 \\
(0.5 \text { to } 1.8)\end{array}$ & $\begin{array}{l}-50 \cdot 7 \% \\
(-63 \cdot 7 \text { to }-37 \cdot 5)\end{array}$ \\
\hline Cholera & $\begin{array}{c}81 \cdot 7 \\
(39 \cdot 1 \text { to } 117 \cdot 0)\end{array}$ & $\begin{array}{c}5 \cdot 1 \\
(2 \cdot 5 \text { to } 7 \cdot 2)\end{array}$ & $\begin{array}{c}45 \cdot 2 \\
(23 \cdot 4 \text { to } 67 \cdot 6)\end{array}$ & $\begin{array}{c}8.7 \\
(4.5 \text { to } 12 \cdot 7)\end{array}$ & $\begin{array}{l}125 \cdot 3 \\
(61 \cdot 1 \text { to } 173 \cdot 4)\end{array}$ & $\begin{array}{c}4.9 \\
(2 \cdot 4 \text { to } 6 \cdot 7)\end{array}$ & $\begin{array}{c}69 \cdot 9 \\
(37 \cdot 7 \text { to } 97 \cdot 0)\end{array}$ & $\begin{array}{c}5.5 \\
\text { (3.0 to } 7.7)\end{array}$ & $\begin{array}{l}-44 \cdot 3 \% \\
(-56 \cdot 8 \text { to }-26 \cdot 6)\end{array}$ \\
\hline Clostridium difficile & $\begin{array}{c}2 \cdot 1 \\
(2 \cdot 0 \text { to } 2 \cdot 3)\end{array}$ & $\begin{array}{c}0.1 \\
(0.1 \text { to } 0.1)\end{array}$ & $\begin{array}{c}2.5 \\
(2 \cdot 4 \text { to } 2 \cdot 7)\end{array}$ & $\begin{array}{c}0.5 \\
(0.4 \text { to } 0.6)\end{array}$ & $\begin{array}{c}20 \cdot 8 \\
\text { (19.9 to 21.9) }\end{array}$ & $\begin{array}{c}0.8 \\
(0.7 \text { to } 0.9)\end{array}$ & $\begin{array}{c}41 \cdot 5 \\
(39 \cdot 1 \text { to } 43 \cdot 9)\end{array}$ & $\begin{array}{c}3.3 \\
\text { (3.0 to } 3.6)\end{array}$ & $\begin{array}{l}99 \cdot 4 \% \\
\text { (84.3 to } 114 \cdot 3)\end{array}$ \\
\hline Cryptosporidiosis & $\begin{array}{c}92 \cdot 4 \\
(68 \cdot 7 \text { to } 125 \cdot 1)\end{array}$ & $\begin{array}{c}5 \cdot 8 \\
(4 \cdot 3 \text { to } 7 \cdot 8)\end{array}$ & $\begin{array}{c}35 \cdot 2 \\
(25 \cdot 9 \text { to } 48 \cdot 2)\end{array}$ & $\begin{array}{c}6.8 \\
(5 \cdot 1 \text { to } 9 \cdot 3)\end{array}$ & $\begin{array}{c}98 \cdot 8 \\
(72 \cdot 8 \text { to } 132 \cdot 7)\end{array}$ & $\begin{array}{c}3.8 \\
(2 \cdot 8 \text { to } 5 \cdot 2)\end{array}$ & $\begin{array}{c}41 \cdot 9 \\
(30 \cdot 0 \text { to } 58 \cdot 4)\end{array}$ & $\begin{array}{c}3 \cdot 3 \\
(2 \cdot 4 \text { to } 4 \cdot 7)\end{array}$ & $\begin{array}{l}-57 \cdot 8 \% \\
(-64 \cdot 8 \text { to }-49 \cdot 6)\end{array}$ \\
\hline $\begin{array}{l}\text { Enteropathogenic } \\
\text { Escherichia coli infection }\end{array}$ & $\begin{array}{c}4.3 \\
(1 \cdot 3 \text { to } 7 \cdot 8)\end{array}$ & $\begin{array}{c}0.3 \\
(0.1 \text { to } 0.5)\end{array}$ & $\begin{array}{c}1.8 \\
(0 \cdot 7 \text { to } 3 \cdot 4)\end{array}$ & $\begin{array}{c}0.3 \\
(0.1 \text { to } 0.7)\end{array}$ & $\begin{array}{c}4.3 \\
(1 \cdot 3 \text { to } 7 \cdot 8)\end{array}$ & $\begin{array}{c}0.2 \\
\text { (0.1 to } 0.3)\end{array}$ & $\begin{array}{c}1.8 \\
(0.7 \text { to } 3 \cdot 4)\end{array}$ & $\begin{array}{c}0.1 \\
(0.1 \text { to } 0.3)\end{array}$ & $\begin{array}{l}-57 \cdot 5 \% \\
(-76 \cdot 5 \text { to }-32)\end{array}$ \\
\hline $\begin{array}{l}\text { Enterotoxigenic } \\
\text { Escherichia coli infection }\end{array}$ & $\begin{array}{l}86 \cdot 0 \\
(61 \cdot 3 \text { to } 114 \cdot 1)\end{array}$ & $\begin{array}{c}5 \cdot 4 \\
(3 \cdot 8 \text { to } 7 \cdot 2)\end{array}$ & $\begin{array}{c}23 \cdot 1 \\
(17 \cdot 0 \text { to } 30 \cdot 4)\end{array}$ & $\begin{array}{c}4 \cdot 4 \\
(3 \cdot 4 \text { to } 5 \cdot 9)\end{array}$ & $\begin{array}{l}134 \cdot 7 \\
(97 \cdot 5 \text { to } 178 \cdot 2)\end{array}$ & $\begin{array}{l}5 \cdot 2 \\
(3 \cdot 8 \text { to } 6 \cdot 8)\end{array}$ & $\begin{array}{c}59 \cdot 2 \\
(44 \cdot 2 \text { to } 77 \cdot 7)\end{array}$ & $\begin{array}{c}4 \cdot 7 \\
(3 \cdot 5 \text { to } 6 \cdot 1)\end{array}$ & $\begin{array}{l}-56 \cdot 0 \% \\
(-63 \cdot 0 \text { to }-46 \cdot 9)\end{array}$ \\
\hline Norovirus & $\begin{array}{c}7 \cdot 3 \\
(2 \cdot 7 \text { to } 11 \cdot 8)\end{array}$ & $\begin{array}{c}0.5 \\
(0.2 \text { to } 0.7)\end{array}$ & $\begin{array}{c}1.8 \\
(0 \cdot 7 \text { to } 3 \cdot 1)\end{array}$ & $\begin{array}{c}0.3 \\
(0.1 \text { to } 0.6)\end{array}$ & $\begin{array}{c}7 \cdot 3 \\
(2 \cdot 7 \text { to } 11 \cdot 8)\end{array}$ & $\begin{array}{c}0.3 \\
(0.1 \text { to } 0.5)\end{array}$ & $\begin{array}{c}1.8 \\
(0 \cdot 7 \text { to } 3 \cdot 2)\end{array}$ & $\begin{array}{c}0.1 \\
(0.1 \text { to } 0.2)\end{array}$ & $\begin{array}{l}-75 \cdot 8 \% \\
(-85 \cdot 1 \text { to }-61 \cdot 6)\end{array}$ \\
\hline $\begin{array}{l}\text { Other Salmonella } \\
\text { infections }\end{array}$ & $\begin{array}{c}19 \cdot 2 \\
\text { (11.1 to 29.1) }\end{array}$ & $\begin{array}{c}1.2 \\
(0.7 \text { to } 1.8)\end{array}$ & $\begin{array}{c}3.8 \\
(1.6 \text { to } 6 \cdot 7)\end{array}$ & $\begin{array}{c}0.7 \\
(0.3 \text { to } 1.2)\end{array}$ & $\begin{array}{c}58 \cdot 9 \\
(42 \cdot 4 \text { to } 77 \cdot 8)\end{array}$ & $\begin{array}{c}2 \cdot 3 \\
(1 \cdot 6 \text { to } 3 \cdot 0)\end{array}$ & $\begin{array}{c}24 \cdot 3 \\
(16 \cdot 0 \text { to } 33 \cdot 3)\end{array}$ & $\begin{array}{c}1.9 \\
(1 \cdot 3 \text { to } 2 \cdot 6)\end{array}$ & $\begin{array}{l}-58 \cdot 9 \% \\
(-65 \cdot 4 \text { to }-51 \cdot 1)\end{array}$ \\
\hline Rotaviral enteritis & $\begin{array}{l}398.9 \\
(334 \cdot 5 \text { to } 464 \cdot 2)\end{array}$ & $\begin{array}{l}24 \cdot 8 \\
(21 \cdot 4 \text { to } 28 \cdot 2)\end{array}$ & $\begin{array}{l}122 \cdot 4 \\
(96 \cdot 6 \text { to } 152 \cdot 1)\end{array}$ & $\begin{array}{l}23 \cdot 5 \\
(20 \cdot 1 \text { to } 27 \cdot 2)\end{array}$ & $\begin{array}{l}477 \cdot 5 \\
\text { (397.9 to } 555 \cdot 1)\end{array}$ & $\begin{array}{l}18 \cdot 5 \\
(15 \cdot 8 \text { to } 21 \cdot 2)\end{array}$ & $\begin{array}{l}176 \cdot 6 \\
(140 \cdot 4 \text { to } 218 \cdot 4)\end{array}$ & $\begin{array}{l}14 \cdot 0 \\
(11 \cdot 4 \text { to } 16 \cdot 6)\end{array}$ & $\begin{array}{l}-63 \cdot 2 \% \\
(-68 \cdot 5 \text { to }-57 \cdot 1)\end{array}$ \\
\hline Shigellosis & $\begin{array}{l}161 \cdot 0 \\
(130 \cdot 0 \text { to } 200 \cdot 3)\end{array}$ & $\begin{array}{l}10 \cdot 0 \\
(8 \cdot 2 \text { to } 12 \cdot 2)\end{array}$ & $\begin{array}{c}33 \cdot 4 \\
(24 \cdot 9 \text { to } 43 \cdot 5)\end{array}$ & $\begin{array}{c}6 \cdot 4 \\
(5 \cdot 1 \text { to } 7 \cdot 9)\end{array}$ & $\begin{array}{l}254 \cdot 2 \\
\text { (207.9 to } 311 \cdot 7)\end{array}$ & $\begin{array}{c}9.9 \\
(8.2 \text { to } 11.9)\end{array}$ & $\begin{array}{c}73.9 \\
(58.9 \text { to } 93 \cdot 8)\end{array}$ & $\begin{array}{c}5 \cdot 8 \\
(4 \cdot 7 \text { to } 7 \cdot 3)\end{array}$ & $\begin{array}{l}-70 \cdot 9 \% \\
(-74 \cdot 4 \text { to }-67 \cdot 3)\end{array}$ \\
\hline No identified aetiology* & $\begin{array}{l}652 \cdot 4 \\
(542 \cdot 4 \text { to } 783 \cdot 1)\end{array}$ & $\begin{array}{l}40 \cdot 6 \\
(35 \cdot 3 \text { to } 46 \cdot 8)\end{array}$ & $\begin{array}{l}215 \cdot 9 \\
(169 \cdot 6 \text { to } 265 \cdot 2)\end{array}$ & $\begin{array}{l}41 \cdot 5 \\
\text { (35.7 to } 48 \cdot 1)\end{array}$ & $\begin{array}{l}1240 \cdot 9 \\
(1096 \cdot 8 \text { to } 1421 \cdot 5)\end{array}$ & $\begin{array}{l}48 \cdot 1 \\
(43 \cdot 5 \text { to } 53 \cdot 5)\end{array}$ & $\begin{array}{c}702 \cdot 9 \\
(619 \cdot 2 \text { to } 796)\end{array}$ & $\begin{array}{l}55 \cdot 6 \\
(51 \cdot 4 \text { to } 60 \cdot 3)\end{array}$ & $\begin{array}{l}-43 \cdot 2 \% \\
(-49 \cdot 3 \text { to }-36 \cdot 1)\end{array}$ \\
\hline \multicolumn{10}{|c|}{ Lower respiratory infections } \\
\hline $\begin{array}{l}\text { Haemophilus influenzae } \\
\text { type B pneumonia }\end{array}$ & $\begin{array}{l}427 \cdot 1 \\
(-39 \cdot 8 \text { to } 853 \cdot 4)\end{array}$ & $\begin{array}{l}19 \cdot 7 \\
(-1.8 \text { to } 39 \cdot 1)\end{array}$ & $\begin{array}{l}108 \cdot 7 \\
(-9 \cdot 9 \text { to } 226 \cdot 9)\end{array}$ & $\begin{array}{l}12 \cdot 0 \\
(-1 \cdot 1 \text { to } 25 \cdot 5)\end{array}$ & $\begin{array}{c}427 \cdot 1 \\
(-39 \cdot 8 \text { to } 853 \cdot 4)\end{array}$ & $\begin{array}{l}12 \cdot 5 \\
(-1 \cdot 2 \text { to } 24 \cdot 8)\end{array}$ & $\begin{array}{l}108 \cdot 7 \\
(-9 \cdot 9 \text { to } 226 \cdot 9)\end{array}$ & $\begin{array}{c}4 \cdot 1 \\
(-0 \cdot 4 \text { to } 8 \cdot 8)\end{array}$ & $\begin{array}{l}-75 \cdot 1 \% \\
(-79 \cdot 1 \text { to }-71 \cdot 2)\end{array}$ \\
\hline Influenza & $\begin{array}{c}36 \cdot 3 \\
(14.2 \text { to } 73 \cdot 5)\end{array}$ & $\begin{array}{c}1.7 \\
\text { (0.7 to } 3.3)\end{array}$ & $\begin{array}{c}15 \cdot 1 \\
\text { (5.7 to 30.4) }\end{array}$ & $\begin{array}{c}1.7 \\
\text { (0.6 to } 3.3)\end{array}$ & $\begin{array}{c}85 \cdot 1 \\
\text { (36.1 to } 156 \cdot 2)\end{array}$ & $\begin{array}{l}2.5 \\
\text { (1.1 to } 4.5)\end{array}$ & $\begin{array}{c}105 \cdot 4 \\
(45 \cdot 3 \text { to } 188 \cdot 1)\end{array}$ & $\begin{array}{c}4.0 \\
(1 \cdot 7 \text { to } 7 \cdot 3)\end{array}$ & $\begin{array}{c}24 \cdot 0 \% \\
\text { (3.4 to } 47 \cdot 7)\end{array}$ \\
\hline $\begin{array}{l}\text { Pneumococcal } \\
\text { pneumonia }\end{array}$ & $\begin{array}{l}652 \cdot 4 \\
(402 \cdot 6 \text { to } 879 \cdot 4)\end{array}$ & $\begin{array}{l}30 \cdot 1 \\
(19 \cdot 0 \text { to } 40 \cdot 2)\end{array}$ & $\begin{array}{l}264 \cdot 0 \\
(155 \cdot 7 \text { to } 365 \cdot 8)\end{array}$ & $\begin{array}{l}29.2 \\
(18.0 \text { to } 39.2)\end{array}$ & $\begin{array}{l}919 \cdot 5 \\
(553 \cdot 1 \text { to } 1320 \cdot 5)\end{array}$ & $\begin{array}{l}26 \cdot 9 \\
(16 \cdot 3 \text { to } 38 \cdot 4)\end{array}$ & $\begin{array}{l}594 \cdot 4 \\
\text { (295.6 to 970.2) }\end{array}$ & $\begin{array}{l}22 \cdot 4 \\
(11.4 \text { to } 35.9)\end{array}$ & $\begin{array}{l}-36 \cdot 1 \% \\
(-52 \cdot 7 \text { to }-19 \cdot 8)\end{array}$ \\
\hline $\begin{array}{l}\text { Respiratory syncytial } \\
\text { virus pneumonia }\end{array}$ & $\begin{array}{l}145 \cdot 1 \\
(82 \cdot 7 \text { to } 228 \cdot 0)\end{array}$ & $\begin{array}{c}6 \cdot 7 \\
(3 \cdot 8 \text { to } 10 \cdot 5)\end{array}$ & $\begin{array}{c}41 \cdot 1 \\
(23 \cdot 0 \text { to } 65 \cdot 5)\end{array}$ & $\begin{array}{c}4.5 \\
(2 \cdot 6 \text { to } 7 \cdot 1)\end{array}$ & $\begin{array}{l}185 \cdot 5 \\
(114 \cdot 4 \text { to } 268 \cdot 6)\end{array}$ & $\begin{array}{c}5 \cdot 4 \\
(3.5 \text { to } 7 \cdot 9)\end{array}$ & $\begin{array}{c}81 \cdot 5 \\
\text { (53.6 to 109.9) }\end{array}$ & $\begin{array}{c}3 \cdot 1 \\
(2 \cdot 0 \text { to } 4 \cdot 2)\end{array}$ & $\begin{array}{l}-55 \cdot 7 \% \\
(-63 \text { to }-47 \cdot 2)\end{array}$ \\
\hline No identified aetiology* & $\begin{array}{l}907 \cdot 2 \\
(407 \cdot 3 \text { to } 1451 \cdot 3)\end{array}$ & $\begin{array}{l}41 \cdot 9 \\
(19.0 \text { to } 66 \cdot 7)\end{array}$ & $\begin{array}{l}476 \cdot 2 \\
(313 \cdot 3 \text { to } 651 \cdot 6)\end{array}$ & $\begin{array}{l}52 \cdot 6 \\
(35 \cdot 2 \text { to } 70 \cdot 2)\end{array}$ & $\begin{array}{l}1803.4 \\
(1211.8 \text { to } 2452 \cdot 5)\end{array}$ & $\begin{array}{l}52 \cdot 7 \\
(35 \cdot 4 \text { to } 71 \cdot 1)\end{array}$ & $\begin{array}{l}1762 \cdot 6 \\
(1385 \cdot 3 \text { to } 2134 \cdot 1)\end{array}$ & $\begin{array}{l}66 \cdot 5 \\
\text { (52.8 to 79.9) }\end{array}$ & $\begin{array}{l}-0 \cdot 8 \% \\
(-19 \cdot 8 \text { to } 22 \cdot 9)\end{array}$ \\
\hline
\end{tabular}

Risk of death from transport injuries was greater than $2 \%$ for men in Afghanistan, Côte d'Ivoire, Cameroon, Ecuador, Gabon, Guinea-Bissau, Equatorial Guinea, Kazakhstan, Mauritania, Nigeria, Oman, Sierra Leone, El Salvador, Thailand, and Uganda. Probability of death from cirrhosis in Myanmar was $1.9 \%$ for men. Tuberculosis stood out in Cambodia, Indonesia, Laos, Myanmar, and Philippines for men. The gap between the probability of death for men and women was particularly large in eastern Europe and central Asia. Mongolia had the highest probability of death in reproductive age for men in these two regions, as a result of unusually high probabilities of interpersonal violence, self-harm, alcohol and drug use, cirrhosis, liver cancer, and tuberculosis. More generally for men, in eastern Europe and central Asia, there were major 
contributions from ischaemic heart disease, self-harm, alcohol and drug use, cirrhosis, and tuberculosis; HIV played an important part in Ukraine and Russia. In north Africa and Middle East, transport injuries and ischaemic heart disease were predominant. For women in the region, breast cancer in all countries and maternal mortality in Sudan and Yemen were also major factors.

In Latin America and Caribbean, there was a large contribution of interpersonal violence in men, with the probability of death exceeding $2 \%$ in Brazil, Colombia, El Salvador, Guatemala, Honduras, and Venezuela. Despite generally high violence in the region, Cuba, Costa Rica, Bolivia, and Peru had low probabilities of death from violence. In men, the probability of death because of HIV/AIDS exceeded 1\% in Belize, Haiti, Saint Vincent and the Grenadines, The Bahamas, Grenada, Guyana, Suriname, and Trinidad and Tobago. Cirrhosis contributed more than $1 \%$ to the probability of death of men in Guatemala, Mexico, and Guyana. Cervical cancer was a larger contributor to the probability of death than was breast cancer in eight countries of Latin America and Caribbean (Bolivia, Ecuador, Guatemala, Nicaragua, Peru, Paraguay, El Salvador, and Venezuela). Probabilities of death for men and women in Afghanistan were more than twice that of other countries in the region; for women, maternal mortality was the largest cause. In Oceania, Samoa and Tonga had much lower probabilities of death than did other countries in the region. Throughout sub-Saharan Africa, there were major contributions for women from maternal mortality, HIV/AIDS, and tuberculosis. For men in the region, HIV/ AIDS, tuberculosis, and transport injuries dominated in most countries. Liver cancer was also a major factor, particularly for men in western sub-Saharan Africa.

Country-specific probabilities of death during middle age The probability of death in middle age (exact age 50 years to exact age 75 years $\left[{ }_{25} \mathrm{q}_{50}\right]$ ) ranged from $10 \cdot 3 \%$ for women in Andorra to 76 · $3 \%$ for men in Lesotho (figure appendix 3). In all countries, ischaemic heart disease and stroke were important contributors to the risk of death in middle age, and were greater for men than for women. Probabilities of death from ischaemic heart disease ranged from $0.8 \%$ in Japan for women to more than $24 \%$ in Belarus for men. In high-income regions, lung cancer was as a major contributor to the risk of death for men. Breast cancer for women and prostate cancer for men also made substantial contributions. Probability of death from liver cancer was greater than 2\% in China, Mongolia, Myanmar, North Korea, South Korea, Taiwan (province of China), Thailand, and Vietnam.

In central Europe, chronic respiratory diseases and cirrhosis made clear contributions in Bulgaria, Croatia, Hungary, Poland, Romania, and Slovenia. In some highincome countries, including Singapore, Argentina, and Uruguay, lower respiratory infections were important causes of death for this age group, more so for men than for women. In southeast Asia, liver cancer, diabetes, and tuberculosis made larger contributions than in many other regions, particularly in Myanmar, Philippines, Laos, Indonesia, and Cambodia. Elsewhere in the region, stroke, ischaemic heart disease, other cardiovascular and circulatory diseases, and chronic respiratory diseases were predominant. In all countries of eastern Europe and central Asia, stroke and ischaemic heart disease were particularly prominent for both sexes. Liver cancer in Mongolia had the highest probability of causing death in the world for this age group.

Egypt had extraordinarily high cirrhosis mortality, particularly from hepatitis $\mathrm{C}$, in middle aged men and women. Deaths caused by diabetes were particularly high in Morocco, Bahrain, Oman, and Qatar. In central Latin America and Caribbean, diabetes made large contributions to causes of death in men and women; the highest probability of death in these regions from diabetes for males was $9.2 \%$ in Trinidad and Tobago and $8.4 \%$ for women in Guyana. Chronic kidney disease was particularly high in El Salvador, Mexico, and Nicaragua; more so for men than for women. ${ }^{75,76}$ In the Caribbean, diabetes, stroke, and ischaemic heart disease accounted for $33.4 \%$ of the probability of death in this age group in Haitian men, and $54.8 \%$ in Guyanese women.

In all the countries of Oceania, diabetes accounted for an extremely large fraction of mortality in middle-aged women. For nearly all countries in sub-Saharan Africa, stroke and other cardiovascular diseases (including cardiomyopathies) were important. HIV/AIDS and tuberculosis, diarrhoea, and lower respiratory infections were also estimated to be important causes in almost every country in the region. The probability of death from liver cancer was high in most countries of western sub-Saharan Africa.

\section{Country-specific leading causes of YLLs}

Worldwide, the top ten causes of YLLs were ischaemic heart disease, lower respiratory infections, stroke, diarrhoea, road injury, HIV/AIDS, preterm birth, malaria, neonatal encephalopathy, and congenital causes (figure 12). The differences between highincome and low-income countries was substantial. Selfharm was the fourth highest cause of YLLs in high-income countries and the 14th in low-income countries. Lung cancer, self harm, Alzheimer's disease and other dementias, cirrhosis, chronic obstructive pulmonary disease, and colorectal cancer were in the top ten causes in high-income countries but not in lowincome countries. Conversely, diarrhoea, malaria, HIV/ AIDS, preterm birth complications, neonatal encephalopathy, and congenital disorders were in the top ten in low-income, but not high-income, regions.

Ischaemic heart disease, stroke, and lung cancer were the top three causes in 32 GBD developed countries. More notable differences in the rankings across highincome countries were self-harm as the second highest 


\begin{tabular}{|c|c|c|c|c|c|c|c|c|c|c|}
\hline & 1 & 2 & 3 & 4 & 5 & 6 & 7 & 8 & 9 & 10 \\
\hline Global & IHD & LRI & Stroke & Diarrhoea & Road injuries & HIV/AIDS & NN preterm & Malaria & NN encephalitis & Congenital \\
\hline Developed & IHD & Stroke & Lung $C$ & Self harm & Alzheimer's & Cirrhosis & COPD & Colorectal C & LRI & Road injuries \\
\hline Developing & LRI & IHD & Stroke & Diarrhoea & HIV/AIDS & NN preterm & Malaria & Road injuries & NN encephalitis & Congenital \\
\hline High-income & IHD & Lung $C$ & Stroke & Alzheimer's & COPD & Self harm & Colorectal C & LRI & Road injuries & Cirrhosis \\
\hline Australasia & IHD & Lung $C$ & Stroke & Self harm & Colorectal C & Alzheimer's & COPD & Road injuries & Breast C & Diabetes \\
\hline Australia & IHD & Lung C & Stroke & Self harm & Alzheimer's & Colorectal C & COPD & Road injuries & Breast C & Diabetes \\
\hline New Zealand & IHD & Lung $C$ & Stroke & Colorectal C & COPD & Self harm & Alzheimer's & Road injuries & Breast C & Congenital \\
\hline High-income Asia Pacific & Stroke & IHD & Self harm & Lung C & LRI & Stomach C & Liver C & Colorectal C & Cirrhosis & COPD \\
\hline Brunei & IHD & Stroke & Diabetes & Road injuries & Congenital & Lung $C$ & LRI & HIV/AIDS & COPD & Colorectal C \\
\hline Japan & Stroke & IHD & LRI & Lung $C$ & Self harm & Stomach C & Liver C & Colorectal C & COPD & Pancreatic C \\
\hline Singapore & IHD & LRI & Stroke & Lung C & Colorectal C & Self harm & CKD & Breast $C$ & COPD & Liver C \\
\hline South Korea & Stroke & Self harm & Lung $\mathrm{C}$ & Liver C & IHD & Stomach C & Cirrhosis & Road injuries & Diabetes & Colorectal C \\
\hline High-income North America & IHD & Lung C & Alzheimer's & COPD & Stroke & Road injuries & Self harm & Cirrhosis & Diabetes & Colorectal C \\
\hline Canada & IHD & Lung C & Alzheimer's & Stroke & Self harm & Colorectal C & COPD & Road injuries & Breast C & Diabetes \\
\hline USA & IHD & Lung C & COPD & Alzheimer's & Stroke & Road injuries & Self harm & Cirrhosis & Diabetes & Colorectal C \\
\hline Southern Latin America & IHD & Stroke & LRI & COPD & Road injuries & Lung $C$ & Congenital & Cirrhosis & Self harm & Colorectal C \\
\hline Argentina & IHD & Stroke & LRI & COPD & Road injuries & Congenital & Lung $C$ & NN preterm & Colorectal C & Self harm \\
\hline Chile & IHD & Stroke & Cirrhosis & Road injuries & Self harm & Stomach C & LRI & Alzheimer's & Congenital & Lung C \\
\hline Uruguay & IHD & Stroke & Lung $C$ & Alzheimer's & COPD & LRI & Road injuries & Self harm & Colorectal C & Congenital \\
\hline Western Europe & IHD & Lung C & Stroke & Alzheimer's & Colorectal C & COPD & Self harm & Cirrhosis & Breast C & LRI \\
\hline Andorra & IHD & Lung $\mathrm{C}$ & Stroke & Alzheimer's & Colorectal C & COPD & LRI & Self harm & HIVIAIDS & Pancreatic C \\
\hline Austria & IHD & Lung $\mathrm{C}$ & Stroke & Alzheimer's & Self harm & Cirrhosis & Colorectal C & COPD & Breast C & Diabetes \\
\hline Belgium & IHD & Lung C & Stroke & Self harm & COPD & Alzheimer's & LRI & Colorectal C & Breast C & Road injuries \\
\hline Cyprus & IHD & Stroke & Lung $C$ & Road injuries & Diabetes & Alzheimer's & Breast C & Colorectal C & COPD & LRI \\
\hline Denmark & IHD & Lung C & Stroke & COPD & Colorectal C & Alzheimer's & Cirrhosis & Self harm & LRI & Diabetes \\
\hline Finland & IHD & Stroke & Alzheimer's & Lung $\mathrm{C}$ & Self harm & Cirrhosis & Colorectal C & Pancreatic C & Falls & Alcohol \\
\hline France & IHD & Lung $\mathrm{C}$ & Stroke & Self harm & Colorectal C & Alzheimer's & Cirrhosis & Breast C & \begin{tabular}{|l|} 
Road injuries \\
\end{tabular} & Other cardio \\
\hline Germany & IHD & Lung C & Stroke & Alzheimer's & Colorectal C & COPD & Cirrhosis & Self harm & Breast C & Pancreatic C \\
\hline Greece & IHD & Stroke & Lung $C$ & Alzheimer's & COPD & Road injuries & Colorectal C & Breast C & LRI & CKD \\
\hline Iceland & IHD & Lung C & Stroke & Alzheimer's & Self harm & Colorectal C & COPD & Breast C & Prostate C & Road injuries \\
\hline Ireland & IHD & Lung C & Stroke & Self harm & COPD & Colorectal C & LRI & Alzheimer's & Breast C & Congenital \\
\hline Israel & IHD & Lung C & Alzheimer's & Diabetes & Stroke & Colorectal C & Road injuries & CKD & Congenital & Breast C \\
\hline Italy & IHD & Stroke & Lung $C$ & Alzheimer's & Colorectal C & COPD & Diabetes & Breast C & Cirrhosis & Road injuries \\
\hline Luxembourg & IHD & Lung C & Stroke & Self harm & COPD & Colorectal C & Cirrhosis & Alzheimer's & Breast $\mathrm{C}$ & Road injuries \\
\hline Malta & IHD & Stroke & Lung C & Colorectal C & Breast $\mathrm{C}$ & COPD & Congenital & LRI & Pancreatic C & Diabetes \\
\hline Netherlands & IHD & Lung C & Stroke & Colorectal C & COPD & Alzheimer's & Breast C & LRI & Self harm & Pancreatic C \\
\hline Norway & IHD & Lung C & Stroke & Alzheimer's & Colorectal C & COPD & Self harm & LRI & Drugs & Breast C \\
\hline Portugal & Stroke & IHD & Lung C & LRI & Colorectal C & Alzheimer's & Cirrhosis & COPD & Stomach C & Road injuries \\
\hline Spain & IHD & Lung C & Stroke & Alzheimer's & Colorectal C & COPD & Cirrhosis & LRI & Breast C & Road injuries \\
\hline Sweden & IHD & Stroke & Lung C & Colorectal C & Self harm & Alzheimer's & COPD & Prostate C & LRI & Breast C \\
\hline Switzerland & IHD & Lung C & Stroke & Alzheimer's & Self harm & Colorectal C & Breast C & Other cardio & COPD & Pancreatic $\mathrm{C}$ \\
\hline UK & IHD & Lung $C$ & Stroke & COPD & Alzheimer's & LRI & Colorectal C & Breast C & Cirrhosis & Self harm \\
\hline England & $\mathrm{IHD}$ & Lung C & Stroke & COPD & Alzheimer's & LRI & Colorectal C & Breast C & Cirrhosis & Self harm \\
\hline Northern Ireland & IHD & Lung C & Stroke & COPD & LRI & Colorectal C & Alzheimer's & Self harm & Breast C & Cirrhosis \\
\hline Scotland & IHD & Lung $C$ & Stroke & COPD & Alzheimer's & Colorectal C & LRI & Cirrhosis & Self harm & Breast C \\
\hline Wales & IHD & Lung C & Stroke & Alzheimer's & COPD & LRI & Colorectal C & Breast C & Cirrhosis & Self harm \\
\hline Central and eastern Europe and central Asia & IHD & Stroke & LRI & Self harm & Cirrhosis & Lung $\mathrm{C}$ & CMP & Road injuries & COPD & Colorectal C \\
\hline Central Asia & IHD & LRI & Stroke & NN encephalitis & Cirrhosis & Congenital & Road injuries & Self harm & NN preterm & Drowning \\
\hline Armenia & IHD & Stroke & Lung C & Diabetes & Road injuries & Cirrhosis & Congenital & LRI & COPD & Breast C \\
\hline Azerbaijan & IHD & LRI & Stroke & NN encephalitis & Congenital & Cirrhosis & Road injuries & NN preterm & Diabetes & TB \\
\hline Georgia & IHD & Stroke & COPD & Cirrhosis & Lung $C$ & Road injuries & LRI & NN encephalitis & Other cardio & Alzheimer's \\
\hline Kazakhstan & IHD & Stroke & Self harm & Road injuries & Cirrhosis & LRI & Congenital & NN encephalitis & Violence & COPD \\
\hline Kyrgyzstan & IHD & Stroke & LRI & Cirrhosis & NN encephalitis & NN preterm & Congenital & \begin{tabular}{|l} 
Road injuries \\
\end{tabular} & COPD & Self harm \\
\hline Mongolia & IHD & Stroke & LRI & Liver C & NN encephalitis & Cirrhosis & Self harm & Congenital & Road injuries & NN preterm \\
\hline Tajikistan & LRI & IHD & NN encephalitis & NN preterm & Diarrhoea & Congenital & Stroke & Drowning & Cirrhosis & Meningitis \\
\hline Turkmenistan & IHD & LRI & Stroke & NN encephalitis & Diarrhoea & Cirrhosis & Congenital & NN preterm & \begin{tabular}{|l} 
Road injuries \\
\end{tabular} & \begin{tabular}{|l} 
Drowning \\
\end{tabular} \\
\hline Uzbekistan & IHD & LRI & Stroke & NN encephalitis & Cirrhosis & Road injuries & Congenital & HTN HD & Drowning & Self harm \\
\hline Central Europe & IHD & Stroke & Lung $C$ & Cirrhosis & COPD & Self harm & Colorectal C & Alzheimer's & HTN HD & Road injuries \\
\hline Albania & IHD & Stroke & LRI & Lung C & Other cardio & Road injuries & COPD & Violence & Stomach C & Congenital \\
\hline Bosnia and Herzegovina & IHD & Stroke & Lung $C$ & CMP & Diabetes & COPD & Colorectal C & Alzheimer's & Self harm & Cirrhosis \\
\hline Bulgaria & IHD & Stroke & COPD & HTN HD & Lung $C$ & Other cardio & Colorectal C & Alzheimer's & Cirrhosis & LRI \\
\hline Croatia & IHD & Stroke & Lung $\mathrm{C}$ & Colorectal C & Cirrhosis & COPD & Alzheimer's & Self harm & Road injuries & HTN HD \\
\hline
\end{tabular}




\begin{tabular}{|c|c|c|c|c|c|c|c|c|c|c|}
\hline Czech Republic & IHD & Stroke & Lung C & Colorectal C & Cirrhosis & Self harm & Alzheimer's & COPD & LRI & Other cardio \\
\hline Hungary & IHD & Stroke & Lung $C$ & Cirrhosis & Colorectal C & Self harm & COPD & Alzheimer's & HTN HD & Breast C \\
\hline Macedonia & Stroke & IHD & Lung C & Diabetes & HTN HD & Colorectal C & COPD & Stomach C & Alzheimer's & Road injuries \\
\hline Montenegro & IHD & Stroke & Lung $C$ & Self harm & Road injuries & CMP & Breast C & Diabetes & Alzheimer's & Colorectal C \\
\hline Poland & IHD & Stroke & Lung C & Self harm & COPD & Cirrhosis & Road injuries & Colorectal C & Alzheimer's & LRI \\
\hline Romania & IHD & Stroke & Cirrhosis & Lung C & LRI & HTN HD & Alzheimer's & CMP & COPD & Colorectal C \\
\hline Serbia & IHD & Stroke & CMP & Lung $C$ & Self harm & Colorectal C & COPD & Alzheimer's & Diabetes & Breast C \\
\hline Slovakia & IHD & Stroke & Lung $C$ & Cirrhosis & Colorectal C & LRI & Self harm & Alzheimer's & Road injuries & Other cardio \\
\hline Slovenia & IHD & Stroke & Lung $C$ & Self harm & Cirrhosis & Colorectal C & Alzheimer's & COPD & CMP & Road injuries \\
\hline Eastern Europe & IHD & Stroke & Self harm & CMP & Cirrhosis & Lung $C$ & Road injuries & LRI & Violence & Alcohol \\
\hline Belarus & IHD & Stroke & Self harm & Lung $C$ & Road injuries & Cirrhosis & Stomach C & COPD & CMP & Alcohol \\
\hline Estonia & IHD & Stroke & Lung C & Self harm & HTN HD & Alzheimer's & Cirrhosis & Alcohol & Colorectal C & CMP \\
\hline Latvia & IHD & Stroke & CMP & Lung $C$ & Self harm & Alzheimer's & Colorectal C & Cirrhosis & Stomach C & Road injuries \\
\hline Lithuania & IHD & Stroke & Self harm & Lung C & Cirrhosis & Alzheimer's & Road injuries & Colorectal C & CMP & Stomach C \\
\hline Moldova & IHD & Stroke & Cirrhosis & LRI & Self harm & Lung C & Road injuries & COPD & Colorectal C & Congenital \\
\hline Russia & IHD & Stroke & CMP & Self harm & Cirrhosis & Lung $C$ & Road injuries & LRI & Violence & Alcohol \\
\hline Ukraine & IHD & Stroke & Cirrhosis & Self harm & HIV/AIDS & Lung C & Road injuries & Colorectal C & Alzheimer's & CMP \\
\hline Latin America and Caribbean & IHD & Violence & Road injuries & Stroke & LRI & Congenital & Diabetes & Cirrhosis & NN preterm & CKD \\
\hline Andean Latin America & LRI & Road injuries & IHD & Congenital & NN preterm & Stroke & F Body Asp & Cirrhosis & NN encephalitis & CKD \\
\hline Bolivia & LRI & F Body Asp & Road injuries & NN preterm & IHD & Congenital & NN encephalitis & Stroke & Cirrhosis & NN sepsis \\
\hline Ecuador & LRI & Road injuries & IHD & Congenital & Violence & Stroke & CKD & $\mathrm{NN}$ preterm & Self harm & Cirrhosis \\
\hline Peru & LRI & IHD & Road injuries & Congenital & Stroke & NN preterm & Cirrhosis & F Body Asp & NN encephalitis & NN sepsis \\
\hline Caribbean & IHD & Stroke & LRI & HIV/AIDS & Road injuries & Diarrhoea & Diabetes & NN preterm & Congenital & NN sepsis \\
\hline Antigua and Barbuda & IHD & Stroke & Diabetes & LRI & Road injuries & HIV/AIDS & NN preterm & Violence & CKD & Congenital \\
\hline Barbados & IHD & Diabetes & Stroke & LRI & CKD & Road injuries & Violence & HTN HD & Breast $C$ & HIV/AIDS \\
\hline Belize & IHD & Diabetes & Violence & Stroke & Road injuries & NN preterm & Congenital & LRI & HIV/AIDS & Self harm \\
\hline Cuba & IHD & Stroke & Lung C & LRI & Self harm & COPD & Road injuries & Colorectal C & Alzheimer's & CKD \\
\hline Dominica & IHD & Stroke & Diabetes & LRI & Road injuries & Violence & NN preterm & CKD & Congenital & HTN HD \\
\hline Dominican Republic & IHD & Road injuries & Stroke & NN preterm & Congenital & LRI & Violence & NN sepsis & Diabetes & CKD \\
\hline Grenada & IHD & Stroke & Diabetes & LRI & Road injuries & HIV/AIDS & Violence & CKD & Self harm & Congenital \\
\hline Guyana & IHD & Stroke & HIV/AIDS & Diabetes & Road injuries & LRI & Congenital & Self harm & Violence & NN preterm \\
\hline Haiti & HIV/AIDS & LRI & Diarrhoea & Stroke & PEM & NN sepsis & IHD & NN preterm & Congenital & NN encephalitis \\
\hline Jamaica & Stroke & Diabetes & Violence & $\mathrm{IHD}$ & NN preterm & CKD & LRI & Congenital & HIV/AIDS & Alzheimer's \\
\hline Saint Lucia & IHD & Stroke & Diabetes & LRI & Violence & Road injuries & HIV/AIDS & NN preterm & CKD & Congenital \\
\hline VCT & IHD & Stroke & Diabetes & NN preterm & Violence & HIV/AIDS & LRI & Road injuries & Congenital & CKD \\
\hline Suriname & IHD & Stroke & NN preterm & Congenital & Diabetes & LRI & Road injuries & HIV/AIDS & Self harm & CKD \\
\hline The Bahamas & IHD & Stroke & Diabetes & HIV/AIDS & Violence & Road injuries & CKD & LRI & HTN HD & NN preterm \\
\hline тто & IHD & Diabetes & Stroke & Violence & Road injuries & HIV/AIDS & LRI & CKD & Congenital & Self harm \\
\hline Central Latin America & Violence & IHD & Road injuries & CKD & Congenital & LRI & Diabetes & Cirrhosis & Stroke & NN preterm \\
\hline Colombia & Violence & IHD & Road injuries & Stroke & Congenital & LRI & COPD & NN preterm & Diabetes & Self harm \\
\hline Costa Rica & IHD & Road injuries & Congenital & CKD & Stroke & Cirrhosis & Violence & Self harm & Stomach C & COPD \\
\hline El Salvador & Violence & IHD & Road injuries & CKD & LRI & Congenital & Alcohol & Cirrhosis & Diabetes & Stroke \\
\hline Guatemala & LRI & Violence & Diarrhoea & NN preterm & IHD & PEM & Congenital & Cirrhosis & Diabetes & Road injuries \\
\hline Honduras & IHD & Violence & Congenital & NN preterm & Stroke & COPD & Diarrhoea & LRI & Road injuries & Cirrhosis \\
\hline Mexico & $\mathrm{IHD}$ & CKD & Diabetes & Cirrhosis & Violence & Road injuries & Congenital & LRI & Stroke & NN preterm \\
\hline Nicaragua & Congenital & LRI & CKD & IHD & NN preterm & Road injuries & Stroke & Cirrhosis & Violence & Diabetes \\
\hline Panama & IHD & Violence & Congenital & Road injuries & Stroke & LRI & CKD & Diabetes & NN preterm & HIV/AIDS \\
\hline Venezuela & Violence & IHD & Road injuries & Stroke & Congenital & Diabetes & CKD & LRI & Self harm & NN preterm \\
\hline Tropical Latin America & IHD & Violence & Stroke & Road injuries & LRI & Congenital & Diabetes & NN preterm & Cirrhosis & COPD \\
\hline Brazil & IHD & Violence & Stroke & Road injuries & LRI & Congenital & Diabetes & Cirrhosis & NN preterm & COPD \\
\hline Paraguay & IHD & Road injuries & Stroke & Congenital & NN preterm & LRI & Violence & Diabetes & CKD & NN encephalitis \\
\hline Southeast and east Asia and Oceania & Stroke & IHD & Road injuries & COPD & Lung $C$ & LRI & Liver C & Congenital & Cirrhosis & Stomach C \\
\hline East Asia & Stroke & IHD & Road injuries & COPD & Lung $C$ & Liver C & Stomach C & Congenital & LRI & Cirrhosis \\
\hline China & Stroke & IHD & Road injuries & COPD & Lung $C$ & Liver C & Stomach C & Congenital & LRI & Cirrhosis \\
\hline North Korea & Stroke & IHD & Lung $\mathrm{C}$ & COPD & Road injuries & Liver C & Stomach C & Self harm & LRI & Congenital \\
\hline Taiwan (province of China) & IHD & Stroke & Liver C & Lung C & Diabetes & Cirrhosis & Self harm & Road injuries & LRI & Colorectal C \\
\hline Oceania & LRI & IHD & Diabetes & Diarrhoea & Congenital & Malaria & NN preterm & Stroke & Road injuries & Asthma \\
\hline FSM & IHD & Diabetes & Stroke & LRI & Road injuries & Congenital & Asthma & Self harm & CKD & COPD \\
\hline Fiji & IHD & Diabetes & Stroke & LRI & Congenital & NN preterm & CKD & Road injuries & Breast C & COPD \\
\hline Kiribati & Stroke & Diabetes & IHD & LRI & Congenital & Road injuries & Diarrhoea & Asthma & Self harm & NN preterm \\
\hline Marshall Islands & IHD & Diabetes & LRI & Stroke & Congenital & NN preterm & Road injuries & CKD & Diarrhoea & Self harm \\
\hline PNG & LRI & IHD & Diarrhoea & Diabetes & Malaria & NN preterm & Congenital & Road injuries & HIV/AIDS & Asthma \\
\hline Samoa & Diabetes & IHD & Stroke & LRI & Congenital & Road injuries & CKD & Violence & Asthma & Self harm \\
\hline
\end{tabular}




\begin{tabular}{|c|c|c|c|c|c|c|c|c|c|c|}
\hline $\begin{array}{l}\text { Solomon Islands } \\
\text { sol }\end{array}$ & IHD & Diabetes & Stroke & LRI & Diarrhoea & Congenital & NN preterm & Asthma & TB & \begin{tabular}{|l} 
Road injuries \\
\end{tabular} \\
\hline Tonga & IHD & Diabetes & LRI & Stroke & $\mathrm{NN}$ preterm & Congenital & Road injuries & Lung $C$ & Breast C & Meningitis \\
\hline Vanuatu & IHD & LRI & Diabetes & Stroke & $\mathrm{NN}$ preterm & Congenital & Road injuries & Diarrhoea & Asthma & TB \\
\hline Southeast Asia & Stroke & IHD & LRI & Road injuries & TB & $\mathrm{NN}$ preterm & Diabetes & Congenital & Cirrhosis & NN encephalitis \\
\hline Cambodia & IHD & LRI & $\mathrm{NN}$ preterm & Stroke & Congenital & Road injuries & NN encephalitis & TB & Diarrhoea & Self harm \\
\hline Indonesia & Stroke & IHD & LRI & TB & Road injuries & $\mathrm{NN}$ encephalitis & Diabetes & Diarrhoea & $\mathrm{NN}$ preterm & Cirrhosis \\
\hline Laos & LRI & NN preterm & IHD & Stroke & Diarrhoea & Road injuries & Congenital & $\mathrm{NN}$ encephalitis & TB & Drowning \\
\hline Malaysia & IHD & Road injuries & Stroke & LRI & Lung $\mathrm{C}$ & Congenital & COPD & Diabetes & HIV/AIDS & CKD \\
\hline Maldives & IHD & Stroke & Congenital & NN encephalitis & $\mathrm{NN}$ preterm & Drowning & COPD & Road injuries & CKD & LRI \\
\hline Myanmar & Stroke & LRI & Cirrhosis & TB & IHD & Malaria & $\mathrm{NN}$ preterm & Road injuries & Lung $\mathrm{C}$ & NN encephalitis \\
\hline Philippines & IHD & LRI & Stroke & TB & NN preterm & Congenital & Diabetes & Violence & Road injuries & NN encephalitis \\
\hline Sri Lanka & IHD & Self harm & Stroke & Diabetes & COPD & Road injuries & Cirrhosis & LRI & Congenital & Violence \\
\hline Thailand & IHD & Road injuries & Stroke & LRI & Liver C & Cirrhosis & CKD & HIV/AIDS & Self harm & Diabetes \\
\hline Timor-Leste & LRI & $\mathrm{NN}$ preterm & Congenital & Diarrhoea & IHD & Stroke & NN encephalitis & Road injuries & Drowning & Maternal \\
\hline Vietnam & Stroke & Road injuries & LRI & Liver C & $\mathrm{NN}$ preterm & IHD & Drowning & Cirrhosis & Congenital & Lung $\mathrm{C}$ \\
\hline South Asia & IHD & LRI & NN encephalitis & NN preterm & Diarrhoea & TB & Stroke & COPD & Road injuries & Self harm \\
\hline Afghanistan & LRI & NN preterm & IHD & Diarrhoea & Congenital & Road injuries & Stroke & Meningitis & Maternal & TB \\
\hline Bangladesh & Stroke & IHD & NN encephalitis & LRI & NN preterm & Drowning & NN sepsis & Cirrhosis & Self harm & Congenital \\
\hline Bhutan & $\overline{\text { IHD }}$ & LRI & NN encephalitis & Stroke & $\mathrm{NN}$ preterm & Road injuries & Congenital & Cirrhosis & COPD & NN sepsis \\
\hline India & IHD & LRI & TB & NN encephalitis & NN preterm & Diarrhoea & Stroke & COPD & Self harm & \begin{tabular}{|l} 
Road injuries \\
\end{tabular} \\
\hline Nepal & LRI & IHD & NN encephalitis & Stroke & Diarrhoea & Self harm & TB & $\mathrm{NN}$ preterm & NN sepsis & COPD \\
\hline Pakistan & LRI & NN encephalitis & Diarrhoea & $\mathrm{IHD}$ & NN preterm & $\mathrm{NN}$ sepsis & Stroke & Meningitis & Congenital & TB \\
\hline North Africa and Middle East & IHD & $\mathrm{NN}$ preterm & Congenital & Stroke & Road injuries & LRI & Cirhosis & COPD & Diabetes & Diarrhoea \\
\hline Algeria & NN preterm & IHD & Stroke & Congenital & Road injuries & Diabetes & LRI & NN encephalitis & CKD & NN sepsis \\
\hline Bahrain & IHD & Road injuries & Diabetes & Self harm & Congenital & Stroke & $\mathrm{NN}$ preterm & Drugs & CKD & Breast C \\
\hline Egypt & IHD & Stroke & Cirrhosis & Congenital & LRI & COPD & Other cardio & $\mathrm{NN}$ preterm & CKD & Road injuries \\
\hline Iran & IHD & $\mathrm{NN}$ preterm & Congenital & Road injuries & Stroke & LRI & Other cardio & HTN HD & COPD & Self harm \\
\hline Iraq & NN preterm & IHD & Congenital & Stroke & LRI & CKD & Road injuries & Violence & Diabetes & Diarrhoea \\
\hline Jordan & Congenital & IHD & $\mathrm{NN}$ preterm & LRI & Stroke & Road injuries & Drowning & Diabetes & CKD & NN encephalitis \\
\hline Kuwait & IHD & Congenital & Road injuries & NN preterm & Stroke & LRI & CKD & HTN HD & Diabetes & Cirrhosis \\
\hline Lebanon & IHD & Congenital & Stroke & Lung $C$ & Road injuries & Diabetes & Breast C & NN preterm & COPD & CKD \\
\hline Libya & IHD & Stroke & Congenital & Road injuries & $\mathrm{NN}$ preterm & Diabetes & LRI & CKD & COPD & Lung $C$ \\
\hline Morocco & NN preterm & IHD & Diabetes & Stroke & LRI & Road injuries & Congenital & NN encephalitis & Drugs & NN sepsis \\
\hline Oman & Road injuries & IHD & Stroke & Diabetes & Congenital & LRI & Other cardio & $\mathrm{NN}$ preterm & Drowning & CKD \\
\hline Palestine & Congenital & IHD & Stroke & LRI & $\mathrm{NN}$ preterm & Road injuries & CKD & Diabetes & Violence & Drugs \\
\hline Qatar & Road injuries & Congenital & IHD & NN preterm & Self harm & Stroke & Diabetes & Falls & Oth mech & Drowning \\
\hline Saudi Arabia & Road injuries & IHD & Congenital & NN preterm & Stroke & CKD & LRI & Falls & NN sepsis & Drugs \\
\hline Sudan & NN preterm & IHD & Congenital & Diarrhoea & LRI & Stroke & Road injuries & Malaria & HIV/AIDS & \begin{tabular}{|l|} 
Vis Leish \\
\end{tabular} \\
\hline Syria & War & IHD & Stroke & Congenital & Road injuries & COPD & $\mathrm{NN}$ preterm & LRI & Endocrine & Typhoid \\
\hline Tunisia & $\mathrm{IHD}$ & Road injuries & Stroke & Congenital & $\mathrm{NN}$ preterm & Lung $C$ & LRI & COPD & Diabetes & CKD \\
\hline Turkey & IHD & Stroke & Lung $C$ & Congenital & COPD & Road injuries & $\mathrm{NN}$ preterm & Diabetes & LRI & \begin{tabular}{|l|} 
Stomach C \\
\end{tabular} \\
\hline UAE & Road injuries & IHD & Congenital & Stroke & Self harm & LRI & Drugs & $\mathrm{NN}$ preterm & Falls & Diabetes \\
\hline Yemen & NN preterm & IHD & Diarrhoea & Congenital & LRI & Stroke & Malaria & Road injuries & Maternal & COPD \\
\hline Sub-Saharan Africa & HIV/AIDS & Malaria & LRI & Diarrhoea & $\mathrm{NN}$ preterm & NN encephalitis & PEM & Congenital & NN sepsis & TB \\
\hline Central sub-Saharan Africa & LRI & Diarrhoea & Malaria & PEM & HIV/AIDS & $\mathrm{NN}$ preterm & Congenital & TB & NN encephalitis & Meningitis \\
\hline Angola & LRI & Diarrhoea & HIV/AIDS & Malaria & Congenital & PEM & NN preterm & $\mathrm{NN}$ encephalitis & TB & \begin{tabular}{|l} 
Road injuries \\
\end{tabular} \\
\hline CAR & HIV/AIDS & LRI & Diarrhoea & Malaria & TB & NN preterm & PEM & $\mathrm{NN}$ encephalitis & Syphilis & Meningitis \\
\hline Republic of Congo & HIV/AIDS & LRI & Malaria & Congenital & Stroke & $\mathrm{NN}$ preterm & Diarrhoea & Measles & NN encephalitis & TB \\
\hline DR Congo & Diarrhoea & LRI & PEM & Malaria & $\mathrm{NN}$ preterm & HIV/AIDS & Congenital & TB & NN encephalitis & Meningitis \\
\hline Equator Guinea & HIV/AIDS & LRI & Malaria & Congenital & Road injuries & Diarrhoea & Stroke & PEM & $\mathrm{NN}$ preterm & NN encephalitis \\
\hline Gabon & HIV/AIDS & LRI & Malaria & Stroke & Road injuries & Congenital & NN encephalitis & TB & IHD & NN preterm \\
\hline Eastern sub-Saharan Africa & HIV/AIDS & LRI & Malaria & Diarrhoea & TB & NN preterm & NN encephalitis & PEM & NN sepsis & Congenital \\
\hline Burundi & Malaria & LRI & Diarrhoea & TB & HIV/AIDS & NN preterm & PEM & NN encephalitis & NN sepsis & Congenital \\
\hline Comoros & LRI & TB & Diarrhoea & NN preterm & Malaria & NN encephalitis & NN sepsis & Stroke & Road injuries & Congenital \\
\hline Djibouti & HIV/AIDS & LRI & Malaria & Diarrhoea & TB & Stroke & NN encephalitis & PEM & $\mathrm{NN}$ preterm & Congenital \\
\hline Eritrea & Diarrhoea & LRI & TB & HIV/AIDS & Malaria & $\mathrm{NN}$ preterm & PEM & Maternal & NN encephalitis & NN sepsis \\
\hline Ethiopia & LRI & Diarrhoea & HIV/AIDS & TB & NN preterm & NN encephalitis & Malaria & NN sepsis & Congenital & Stroke \\
\hline Kenya & HIV/AIDS & LRI & Diarrhoea & TB & $\mathrm{NN}$ preterm & NN encephalitis & Malaria & Congenital & NN sepsis & PEM \\
\hline Madagascar & LRI & Diarrhoea & Stroke & NN preterm & PEM & Syphilis & Malaria & NN sepsis & Congenital & Meningitis \\
\hline Malawi & HIV/AIDS & LRI & Diarrhoea & PEM & TB & $\mathrm{NN}$ preterm & Malaria & Congenital & NN encephalitis & Meningitis \\
\hline Mauritius & Diabetes & IHD & Stroke & CKD & Cirrhosis & LRI & Road injuries & Self harm & HTN HD & Congenital \\
\hline Mozambique & HIV/AIDS & Malaria & LRI & Diarrhoea & TB & NN sepsis & NN encephalitis & Syphilis & $\mathrm{NN}$ preterm & Road injuries \\
\hline
\end{tabular}




\begin{tabular}{|c|c|c|c|c|c|c|c|c|c|c|}
\hline Rwanda & LRI & HIV/AIDS & Malaria & Diarrhoea & NN preterm & NN encephalitis & NN sepsis & TB & Road injuries & PEM \\
\hline Seychelles & IHD & Stroke & LRI & HTN HD & Cirrhosis & Drowning & Road injuries & Self harm & Congenital & CKD \\
\hline Somalia & Diarrhoea & LRI & Malaria & TB & PEM & $\mathrm{NN}$ preterm & Meningitis & NN encephalitis & Tetanus & NN sepsis \\
\hline South Sudan & LRI & Diarrhoea & HIV/AIDS & TB & PEM & Syphilis & Meningitis & Maternal & Malaria & NN preterm \\
\hline Tanzania & HIV/AIDS & 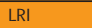 & Malaria & Diarrhoea & TB & Congenital & PEM & NN encephalitis & Syphilis & NN sepsis \\
\hline Uganda & HIV/AIDS & Malaria & LRI & Diarrhoea & $\mathrm{NN}$ preterm & NN encephalitis & NN sepsis & TB & PEM & Road injuries \\
\hline Zambia & HIV/AIDS & Malaria & LRI & Diarrhoea & PEM & TB & NN encephalitis & NN sepsis & Congenital & Meningitis \\
\hline Southern sub-Saharan Africa & HIV/AIDS & LRI & Diarrhoea & TB & Violence & Stroke & NN preterm & Road injuries & IHD & NN encephalitis \\
\hline Botswana & HIV/AIDS & TB & LRI & Diarrhoea & Road injuries & Self harm & NN preterm & NN encephalitis & Maternal & Violence \\
\hline Lesotho & HIV/AIDS & TB & Diarrhoea & LRI & $\mathrm{NN}$ preterm & Violence & NN encephalitis & Self harm & Stroke & Road injuries \\
\hline Namibia & HIV/AIDS & TB & LRI & Diarrhoea & Stroke & Self harm & Road injuries & NN preterm & $\mathrm{IHD}$ & Violence \\
\hline South Africa & HIV/AIDS & LRI & TB & Diarrhoea & Violence & Stroke & Road injuries & IHD & Diabetes & NN preterm \\
\hline Swaziland & HIV/AIDS & LRI & Diarrhoea & TB & Road injuries & NN preterm & Self harm & Violence & Stroke & NN encephalitis \\
\hline Zimbabwe & HIV/AIDS & LRI & Diarrhoea & TB & $\mathrm{NN}$ preterm & NN encephalitis & Stroke & PEM & Malaria & Meningitis \\
\hline Western sub-Saharan Africa & Malaria & LRI & HIV/AIDS & Diarrhoea & $\mathrm{NN}$ preterm & NN encephalitis & Sickle & Road injuries & PEM & NN sepsis \\
\hline Benin & Malaria & LRI & HIV/AIDS & Diarrhoea & $\mathrm{NN}$ preterm & NN encephalitis & Congenital & NN sepsis & Road injuries & PEM \\
\hline Burkina Faso & Malaria & LRI & Diarrhoea & $\mathrm{NN}$ preterm & Congenital & Meningitis & NN encephalitis & NN sepsis & Road injuries & HIV/AIDS \\
\hline Cameroon & HIV/AIDS & LRI & Malaria & Diarrhoea & Road injuries & NN preterm & NN encephalitis & Congenital & PEM & NN sepsis \\
\hline Cape Verde & Stroke & IHD & Congenital & LRI & Stomach C & NN encephalitis & Liver C & Violence & COPD & NN preterm \\
\hline Chad & Diarrhoea & LRI & Malaria & HIV/AIDS & PEM & NN preterm & NN encephalitis & Meningitis & Tetanus & Congenital \\
\hline Côte d'Ivoire & LRI & HIV/AIDS & Malaria & Diarrhoea & $\mathrm{NN}$ preterm & NN encephalitis & NN sepsis & Road injuries & Congenital & PEM \\
\hline Ghana & Malaria & LRI & HIV/AIDS & NN sepsis & $\mathrm{NN}$ preterm & PEM & NN encephalitis & Stroke & Road injuries & Congenital \\
\hline Guinea & Malaria & LRI & Diarrhoea & HIV/AIDS & $\mathrm{NN}$ preterm & NN encephalitis & PEM & NN sepsis & Meningitis & Congenital \\
\hline Guinea-Bissau & Malaria & HIV/AIDS & $\overline{L R I}$ & Diarrhoea & NN preterm & PEM & NN encephalitis & Meningitis & Road injuries & NN sepsis \\
\hline Liberia & Malaria & LRI & Diarrhoea & HIV/AIDS & $\mathrm{NN}$ preterm & NN encephalitis & NN sepsis & PEM & Congenital & Stroke \\
\hline Mali & Malaria & Diarrhoea & LRI & PEM & $\mathrm{NN}$ preterm & $\mathrm{NN}$ encephalitis & NN sepsis & Meningitis & Congenital & HIV/AIDS \\
\hline Mauritania & LRI & Malaria & Diarrhoea & NN encephalitis & $\mathrm{NN}$ preterm & Road injuries & NN sepsis & Congenital & Stroke & Maternal \\
\hline Niger & Malaria & Diarrhoea & LRI & PEM & $\mathrm{NN}$ preterm & Meningitis & NN encephalitis & Congenital & NN sepsis & TB \\
\hline Nigeria & Malaria & LRI & HIV/AIDS & Sickle & Road injuries & NN preterm & NN encephalitis & Diarrhoea & PEM & NN sepsis \\
\hline São Tomé and Príncipe & LRI & Malaria & Stroke & $\mathrm{NN}$ preterm & NN encephalitis & NN sepsis & Congenital & PEM & Diarrhoea & IHD \\
\hline Senegal & Malaria & LRI & Diarrhoea & NN preterm & NN encephalitis & NN sepsis & HIV/AIDS & Congenital & Road injuries & PEM \\
\hline Sierra Leone & Malaria & LRI & HIV/AIDS & PEM & NN preterm & Diarrhoea & NN encephalitis & Congenital & NN sepsis & Meningitis \\
\hline The Gambia & Malaria & LRI & Diarrhoea & Congenital & $\mathrm{NN}$ preterm & HIV/AIDS & NN sepsis & NN encephalitis & Road injuries & PEM \\
\hline Togo & Malaria & LRI & HIV/AIDS & Diarrhoea & $\mathrm{NN}$ preterm & NN encephalitis & Congenital & PEM & NN sepsis & Road injuries \\
\hline
\end{tabular}

Figure 12: Top ten causes in 2013 of years of life lost by location

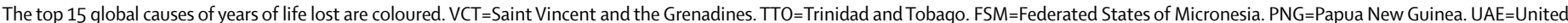
Arab Emirates. CAR=Central African Republic. STP=São Tomé and Princípe. IHD=ischaemic heart disease. LRI=lower respiratory infections. Road inj=road injuries. NN Preterm=preterm birth complications. NN enceph=neonatal encephalitis. Congenital=congenital disorders. $C=$ cancer. $C O P D=$ chronic obstructive pulmonary disease. $C K D=$ chronic kidney disease. $C M P=$ cardiomyopathies Other cardio=other cardiovascular disease. Drugs=drug use disorders. Alcohol=alcohol use disorders. Violence=interpersonal violence. $\mathrm{HTN} H \mathrm{HD}=\mathrm{hypertensive} \mathrm{heart} \mathrm{disease.} \mathrm{F}$ body asp=pulmonary aspiration and foreign body in airway. NN sepsis=neonatal sepsis. PEM=protein-energy malnutrition. TB=tuberculosis. Vis leish=visceral leishmaniasis. Other mech=other mechanical forces. Endocrine $=$ endocrine, metabolic, blood, and immune disorders. Maternal=maternal disorders. Sickle=sickle cell disorders .

cause of YLLs in South Korea, Alzheimer's disease and other dementias as the third highest cause in Canada, Finland, and Israel, and lower respiratory infections as the second cause in Singapore and the third highest cause in Argentina and Japan. Cirrhosis was the third highest cause in Chile. Colorectal cancer was a top five cause in 13 high-income countries and diabetes was in three high-income countries.

In central Europe, eastern Europe, and central Asia, ischaemic heart disease and stroke dominated but in Bosnia and Herzegovina, Serbia, Latvia, and Russia cardiomyopathies were also in the top five. As a result of higher child mortality in these regions, Azerbaijan, Kyrgyzstan, Mongolia, Tajikistan, Turkmenistan, and Uzbekistan had preterm, or neonatal encephalopathy, in the top five causes. In eastern Europe, five causes (ischaemic heart disease, stroke, self-harm, cirrhosis, and road injury) made up $49.7 \%$ of YLLs $(95 \%$ UI
$48 \cdot 5-51 \cdot 3$; or $29 \cdot 3$ million $[28 \cdot 5-30 \cdot 2])$. In Latin America and Caribbean, more variation exists in the leading cause of YLLs. Lower respiratory infections were the leading cause in Bolivia, Peru, Guatemala, and Ecuador; HIV/AIDS was in Haiti; interpersonal violence was in Colombia, El Salvador, and Venezuela; stroke was in Jamaica; congenital anomalies were in Nicaragua, and ischaemic heart disease was in the rest. Road injury was in the top five for 17 of 29 countries. Diabetes was also in the top five for 13 countries. Chronic kidney disease was in the top five for Barbados, Costa Rica, El Salvador, Mexico, and Nicaragua. Perhaps most unusually, interpersonal violence was in the top five causes in 15 countries in the region, but only one country outside Latin America and Caribbean, namely South Africa.

In east Asia, the top five causes of YLLs, in order, were stroke, ischaemic heart disease, road injury, chronic obstructive pulmonary disorder, and lung cancer. These are 
almost the same top five causes as in USA: the only difference is Alzheimer's disease and other dementias, which was fourth and road injury was sixth, providing evidence of epidemiological convergence between east Asian countries and some high-income countries. In Oceania, ischaemic heart disease, lower respiratory infections, diabetes, and diarrhoea were important. In Papua New Guinea, malaria was also a top five cause. Southeast Asia as a whole, Indonesia, Myanmar, and Philippines have tuberculosis as a top five cause of YLLs. Road injury was a top five cause in Indonesia, Malaysia, Thailand, and Vietnam. Cirrhosis was in the top five in Myanmar and liver cancer in Thailand and Vietnam. Among the countries of south Asia, the leading causes are a mix of neonatal causes and ischaemic heart disease, lower respiratory infections, and stroke in most countries. Tuberculosis was the third highest cause in India.

In north Africa and Middle East, ischaemic heart disease and stroke, preterm birth complications, congenital anomalies, and road injury were prominent leading causes of YLLs. In four countries-Oman, Qatar, Saudi Arabia, and United Arab Emirates-road injury was the leading cause of YLLs. Cirrhosis was the third highest cause of YLLs in Egypt. Self-harm was in the top five in Bahrain, Qatar, and United Arab Emirates. The profile of leading causes of YLLs in sub-Saharan Africa was greatly different from the rest of the world with the exception of Cape Verde, Mauritius, and Seychelles. HIV/ AIDS was the leading cause in 18 countries. Malaria was the leading cause in 14 countries. Lower respiratory infection was the leading cause in Angola, Comoros, Ethiopia, Madagascar, Rwanda, South Sudan, Côte d'Ivoire, Mauritania, and São Tomé and Princípe. Diarrhoea was the leading cause in D R Congo, Eritrea, Somalia, and Chad. Tuberculosis was in the top five causes in 18 countries. Violence was the fifth highest cause in South Africa. Road injury was the fifth highest cause in Equatorial Guinea, Gabon, Botswana, Swaziland, Cameroon, and Nigeria.

\section{Discussion \\ Main findings}

The GBD 2013 incorporates many new datasets for cause of death, particularly from China, and new data for 155 other countries. Compared with the GBD 2010, it provides the most comprehensive and up-to-date assessment of causes of death. The results for the GBD 2013 are based on re-estimation of all causes from 1990 to 2013, and thus supersede all previously published GBD time series (panel). Publication of country-level results provides many opportunities for comparing a country's performance with that of its peers.

On the broadest level, our analysis of 240 causes of death for 188 countries confirms that global life expectancy at birth has continued to improve over the past 23 years and these improvements are driven largely by falls in diarrhoea, lower respiratory infections, and neonatal causes in low-income countries, and decreases in cardiovascular diseases and some cancers in middleincome and high-income countries. HIV/AIDS has had a large enough effect to negate progress made in other causes contributing to decreases in life expectancy, particularly in southern sub-Saharan Africa.

This general progress masks enormous heterogeneity across countries and age groups. Even within regions, substantially different mortality, leading causes of death, and trends exist. Outside sub-Saharan Africa, premature mortality is dominated by relatively few causes including ischaemic heart disease, stroke, lower respiratory infections, road injury, diarrhoea, preterm birth complications, neonatal encephalopathy, congenital anomalies, tuberculosis, chronic obstructive pulmonary disease, cirrhosis, self-harm, and lung cancer. In addition to these common causes, great regional and country variation exists, such as the dominant role of interpersonal violence in most countries of central Latin America and Brazil.

Our study points to extraordinary epidemiological progress: global age-standardised death rates fell significantly for 157 of 240 causes from 1990 to 2013. The largest decreases were for some of the major communicable diseases including diarrhoeal diseases, lower respiratory infections, tuberculosis, and measles. Age-standardised rates for many non-communicable causes are also falling. At the same time, numbers of deaths from 115 of these 240 causes, have increased, driven by both growth in population and shifts in the population age-structure towards older ages. For a further 58 causes, changes in the age-standardised death rate over the 23 year period were not statistically different from no change. For some of these causes, sparse data might have contributed to wide UIs and in other cases uncertainty might have arisen from inconsistent coding across countries. However, eight specific causes account for more than 100000 deaths and their age-standardised death rates have increased significantly since 1990: HIV/AIDS, liver cancer caused by hepatitis $\mathrm{C}$, pancreatic cancer, atrial fibrillation and flutter, drug use disorders, diabetes, chronic kidney disease, and sickle cell disorders. Of these causes, three (HIV/AIDS, diabetes, and chronic kidney disease) account for more than a half a million deaths each. HIV/AIDS, however, has been decreasing as a cause of death since 2005. These causes, which run counter to an extraordinary global trend towards lower agestandardised death rates, deserve special attention.

The rise and subsequent fall of HIV/AIDS is well known as is the rise in diabetes. Increases for atrial fibrillation and flutter, pancreatic cancer, drug use disorders, and chronic kidney disease have received far less global attention. Drug use disorders and chronic kidney diseases cause many more deaths in some regions and countries than in others. Nevertheless, they are important emerging global 
challenges that show the potential adverse effects of some behaviours and socioeconomic developments. In view of the important behavioural component for some of these causes, there is potentially an important role for public health policy and resources to modify these causes of death. These diseases, particularly HIV/AIDS and drug use disorders are also subject to social stigmatisation, which adds an important challenge for effective policy interventions. Although global age-standardised death rates have increased for very few causes, there is remarkable and important variation in trends across countries such that causes with falling global age-standardised rates are increasing in some countries-for example, ischaemic heart disease in China.

\section{Convergence or divergence?}

Ambitious goals have been set for maternal and child mortality, ${ }^{8486}$ such as the end of preventable maternal and child death in a generation. The Lancet Commission Global health 2035: a world converging within a generation has argued that a grand convergence in health is possible between high-income, middle-income, and low-income countries. ${ }^{24}$ Trends in the past 23 years provide an important starting point for framing how great a challenge achieving these aspirations will be and the political will and financial resources required. Part of the answer depends on how the goals are framed-for example, what does convergence mean? In the development literature on economic convergence, ${ }^{87-89}$ convergence has been framed in terms of poverty rates or in terms of income inequality measured by the Gini coefficient or other measures of inequality. Work on convergence in life expectancy has tended to focus on measures of absolute difference ${ }^{90-92}$ rather than relative difference. ${ }^{93}$ We found unequivocal divergence in mortality rates for women aged 25-39 years and older than 80 years and for men aged 20-44 years and 65 years and older, similar to previous estimates of divergence of life expectancy at birth since the 1980s. ${ }^{94}$ In these age groups, both the Gini coefficient and the mean absolute difference in death rates are rising. In all other age groups, except girls aged 10-14 years, relative inequality is increasing but the absolute gap is narrowing. Framing a grand convergence as simply achieving a reduction in the differences in mortality rates across countries might not be sufficiently ambitious to meet the goals of many national policy makers. If mortality decreases in all countries by the same percent per year, absolute difference will decrease and relative differences will stay constant. For age groups in which global relative and absolute differences in death rates are diverging, extraordinary efforts will be needed to achieve laudable goals such as a grand convergence. If convergence includes reducing the ratio of the highest to lowest death rates, even for under-5 mortality, major new efforts will be needed to have faster percent decreases in countries with higher mortality.

\section{Panel: Research in context}

\section{Systematic review}

The GBD 2013 assessment of causes of death is a major improvement in the evidence base compared with GBD 2010 through the inclusion of new data from vital registration systems, verbal autopsy studies, maternal mortality surveillance, injury surveillance and other sources. Through the inclusion of sub-national data on China, Mexico, and UK the evidence base for causes of death has been greatly expanded. Redistribution algorithms for ill-defined causes of death used to enhance the comparability of data were based on new statistical models. GBD 2013 also benefits from several improvements in the methods used to estimate allcause mortality and specific causes of death such as HIV/AIDS GBD 2013 provides a more up-to-date and comprehensive assessment of causes of death than do other studies of cause of death in particular age groups (CHERG), for particular causes (GLOBOCAN), ${ }^{83}$ and previous GBD analyses (GBD 2010)..$^{2-8}$

\section{Interpretation}

This study provides a comprehensive description of mortality levels and patterns worldwide, and provides the evidence to assess progress of global development goals, including control of non-communicable diseases, and priorities for further global health and development debates. Because the study provides a complete re-analysis of trends for each cause from 1990 to 2013, it supersedes the results of the GBD 2010 study. This is the first time that country-specific results for all 188 countries with populations of more than 50000 people have been comprehensively published. Country-specific data provide the opportunity to examine the extent to which epidemiological convergence is occurring across countries.

Arguments that convergence is technically and financially feasible are grounded on the rapid improvements of some countries. ${ }^{24}$ For example, from 1990 to 2013, 13 countries (all low-income), achieved increases in life expectancy greater than 10 years (appendix pp 141-151). The real challenge is whether the strategies to decrease mortality used by these countries are generalisable or transferable to those countries who are making the least progress. The Lancet Commission on global health 2035 drew attention to the four Cs (Cuba, Costa Rica, Chile, and China). Life expectancy has improved faster than the global aggregate trend in China and Chile in the past 23 years.

The good news is that some countries that were low-income in 1990 have achieved remarkable progress in the past 23 years-for example, in Nepal, life expectancy has increased by 12.16 years since 1990 , reaching 70.64 years in 2013 for both sexes combined (appendix pp 141-151). Other examples of improvements greater than 12 years for both sexes combined include Rwanda, Ethiopia, Niger, Maldives, Timor-Leste, and Iran. Because
For CHERG estimates see http://cherg.org/main.html 
the Rwandan genocide occurred after 1990, the progress from the peak of mortality during the genocide until 2013 is even larger, 49.63 years. Studies have already assessed progress in Bangladesh, Ethiopia, and Niger, particularly in reducing child mortality. ${ }^{95}$ Further study of these countries might provide insights about how to achieve low mortality, including the role of development assistance for health, rapid economic growth, and addressing chronic challenges such as famines. Simple assessments built up from individual technology analyses, such as the Disease Control Priorities-2, ${ }^{96}$ assume a high-level of health system efficiency and contextual factors that enable technology to be delivered such as levels of maternal education. Plans to achieve a grand convergence in the face of diverging mortality will need to take into account low levels of health system efficiency and low levels of health system resourcing in some countries and the greater efforts needed to achieve high intervention coverage in low-income countries with inadequate primary health-care systems and low levels of educational attainment. The challenge of improving health system management, particularly locally, is a crucial component of the future plans.

The analysis of average relative difference between countries and average absolute difference between countries by cause (data not shown) shows the general pattern that many communicable, maternal, and neonatal causes, along with war and natural disasters, are highly unequal across countries; almost all have average relative differences of more than 50\%. Among the non-communicable disease categories, mental and substance use disorders is the only cause with a mean relative difference greater than $40 \%$. Following the more stringent criteria for convergence-in which global rates and the Gini coefficient are both falling-only neoplasms and chronic respiratory diseases are converging. As more countries go through the epidemiological transition, it seems likely that cross-country inequalities or relative differences for communicable causes will rise and inequalities for non-communicable causes will narrow. Narrowing inequalities across countries will not necessarily narrow inequalities for non-communicable disease within countries. Because mortality exponentially rises with age, at least after age 50 years, relative differences at older ages, when mortality becomes concentrated, tend to be small. Causes such as diabetes, chronic kidney disease, and alcohol and drug use disorders-for which global death rates are rising and inequality is increasing-are exceptions to this general pattern.

\section{Non-communicable diseases}

For the age-standardised death rates by cause for each country see http://vizhub.healthdata. org $/ \mathrm{cod}$
Age-standardised death rates for cardiovascular and circulatory diseases have fallen in high-income and many middle-income countries since 1990. Rapid falls have occurred in some countries. For example, five countries (Israel, Denmark, Norway, South Korea, and UK), had at least a $65 \%$ decrease in age-standardised death rates for ischaemic heart disease. Many other countries have had decreases of $40-65 \%$. Age-adjusted death rates caused by haemorrhagic stroke fell by three-quarters in South Korea.

The ageing and growth of populations has led to an increase in the total number of cardiovascular deaths, accounting for almost a third of all deaths globally in 2013. Ischaemic heart disease, ischaemic stroke, and haemorrhagic stroke continue to cause most cardiovascular and circulatory deaths in almost all countries. Some Balkan countries are an exception; cardiomyopathy was a leading cause of death, possibly as a result of alcohol exposure or local patterns of garbage codes. ${ }^{97}$ Additional studies are needed to establish whether this finding is driven by medical certification practices or is related to alcohol or some other factor. ${ }^{97}$ Age-standardised death rates for atrial fibrillation and flutter and peripheral vascular disease have increased, possibly because of increased awareness of these conditions or better survival from cardiovascular diseases that share the same risk factors. Much uncertainty remains for trends in mortality caused by rheumatic heart disease, partly because endemic populations are concentrated within poorer subnational regions where data collection is limited and rheumatic heart disease might not always be coded as the underlying cause of death..$^{98}$ Efforts to benchmark changes in cardiovascular and circulatory diseases will benefit from increasing access to verbal autopsy in India and sub-Saharan Africa, household surveys focused on chronic diseases, and improvements in electronic health records.

Generally, cancer deaths are increasing but age-standardised cancer death rates are falling. Some cancer-related risk factors, such as tobacco consumption, have decreased, but others, such as obesity, have increased. The substantial general fall in cancers require further explanation. Death rates for five cancers increased (non-Hodgkin lymphoma, mesothelioma, kidney cancer, pancreatic cancer, and multiple myeloma); some explanations, such as the potential link between the rise of diabetes and pancreatic cancer might account for some of these reversals. Because of different rates of decrease for other sites, the mix of cancers is steadily changing, particularly in low-income regions, such as the relative importance of breast cancer compared with cervical cancer. These local changes have important implications for the development of cancer care programmes and training. Because of the strong relation between cancer mortality and age, ageing of the world's population is the most important driver of the rising number of cancer deaths in most countries. Most countries can expect to have to deal with more patients who need diagnosis, treatment, and palliation in coming years.

\section{Alzheimer's disease and other dementias}

We used a substantially different approach to estimate Alzheimer's disease and other dementia mortality in the GBD 2013 by focusing on studies of prevalence and 
using data from countries with the highest death to prevalence ratios in 2013 to estimate mortality in other regions and back in time. This change greatly lowers the increase compared with GBD 2010 in the agestandardised death rate for dementia although the numbers of dementia deaths nevertheless increased. Lower increases in the age-standardised rate were because the meta-regression of prevalence studies did not show a rapidly rising trend; one study, reported decreases in age-specific rates, although our overall assessment suggests a slight increase in age-specific rates. ${ }^{99}$ Even in high-income countries with complete medical certification of causes of death, we argue that dementia was systematically underestimated as a cause of death in earlier periods. Other studies, such as the National Mortality Followback Study in USA, support this idea. ${ }^{100}$ Dementia deaths might have been misclassified into categories such as senility. Our garbage code redistribution algorithms for this broad category might have under-allocated dementia deaths in earlier periods. For future research, we may want to more carefully trace to which garbage codes dementia deaths might have been assigned using hospital linkage or other approaches.

The other effect of using this approach is that we estimated considerably more dementia deaths in middle-income countries than in the GBD 2010. Prevalence studies suggest dementia occurs in these countries although it is rarely recorded on a death certificate as a cause of death. Our overall conclusion is that dementia is more common worldwide and that numbers are increasing because of population ageing with only a small component of the increase caused by rising age-specific rates. The analysis of dementia will benefit from further population-based prevalence surveys, especially with repeated measurement over time using standardised definitions and methods. As further studies of this type become available and incorporated into the GBD, our estimates of dementia burden might be substantially revised. Trends in the category Alzheimer's disease and other dementias might mask upward trends in Alzheimer's and downward trends in vascular dementia; however, these disorders are difficult to tell apart in population-based prevalence studies and cause of death data. Nevertheless, our finding that the number of dementia deaths is increasing implies that governments should remain concerned about the rising demands for care that will come with population ageing even if future rates do not increase substantially.

\section{Diarrhoea and lower respiratory infections}

We report that the distribution of the causes of diarrhoea is different around the world. The distribution of pathogens has also changed significantly since 1990-for example, almost 50\% (20343 [9054-41216] deaths) of all cholera deaths in children occur in sub-Saharan Africa. Because we used GEMS data to estimate relative risks, it is perhaps not surprising that our results are comparable to their findings. ${ }^{57}$ The population attributable fraction for pathogens such as Campylobacter, Shigella, and Salmonella were not significant in some countries and some ages. Because of the nature of case notification data, we had to estimate all types of cholera and were unable to breakdown cholera into O1, non-O1, and O-139.

In high-income countries, $C$ difficile is an important threat that has increased during the past two decades. $65 \%$ (744413 deaths) of unexplained diarrhoea in people older than 5 years is an important knowledge gap. Although the new counterfactual approach is successful for estimating attributable death empirically and adjusts for the overall pathogen load in the country, it still suffers from limitations such as the potential low sensitivity of diagnostic tests. Some pathogens are more prevalent in controls than in cases, which might present a distorted causal picture because of continuous shedding of pathogen long after the acute phase. ${ }^{101-105}$ These findings could also suggest a protective effect of infection from one or more pathogens against other pathogens or could be simply caused by a differential decrease in the sensitivity of diagnostic tests (for other pathogens) where diarrhoea presents assuming a single pathogen caused the diarrhoea. More sensitive diagnostic tests help to improve sensitivity but at the price of decreased specificity because of contamination and post-diarrhoea pathogen excretion. Follow-up studies with multiple measurements of pathogens in children during healthy and diarrhoea periods could help to elucidate the true causal associations. Better case definition and more strict criteria for pathogens such as excluding recent cases of diarrhoea could decrease exposure misclassifications.

Our estimates of the fraction of under-5 lower respiratory infection deaths attributable to the four causes of pneumonia (pneumococcus, $H$ influenzae B, respiratory syncytial virus, and influenza) are much the same as previous estimates, with pneumococcus and $H$ influenzae $\mathrm{B}$ the predominant causes. ${ }^{63,64,106-108}$ The large fraction of lower respiratory infection attributable to pneumococcus and $H$ influenzae $\mathrm{B}$, particularly in low-income regions where the absolute burden is highest, shows the potential benefit of continuing to scale up pneumococcal conjugate and $H$ influenzae B vaccination. We calculated the contribution of each cause with a counterfactual approach. This approach means that they do not add up to $100 \%$ but also that there might be overlap; for example, death from lower respiratory infection might involve viral and bacterial co-infection. These results should also be interpreted with caution because of the data used to generate these estimates. Data for cause are sparse and prone to several biases, which is shown in the large UIs.

Estimates of the mortality burden of pneumococcal pneumonia in children rely on data from vaccine probe studies, which showed that disease in infants fell after 
pneumococcal conjugate vaccination; there are no sensitive diagnostic tests to detect non-bacteraemic pneumococcal pneumonia in children. To calculate burden in the absence of a diagnostic assay, the pneumococcal conjugate vaccination probe studies assumed a vaccine efficacy against non-bacteraemic pneumonia caused by vaccine types equal to that of protection from vaccine type bacteraemia (75\%). Data from a large randomised trial of pneumococcal conjugate vaccination in adults confirmed efficacy against bacteraemic pneumonia of $75 \%$, but efficacy against non-bacteraemic pneumonia was only $45 \% .{ }^{109}$ If similar efficacy estimates are applied to infants, then the contribution of the pneumococcus to pneumonia mortality in infants could be as high as 63\% (166324 deaths). Because data were sparse, we did not estimate the fraction of deaths caused by $H$ influenzae B among people aged 5 years and older. The before-and-after vaccine efficacy studies used to estimate the burden of pneumococcus were limited to high-income settings. These types of studies might also be biased because of underlying temporal trends in hospital admissions for lower respiratory infection. Furthermore, the only variation included for pneumococcus and $H$ influenzae B is a result of differences in vaccination coverage.

The observational studies used for respiratory syncytia virus and influenza were based on case series data from predominantly tertiary-level hospitals, which might not be representative of the underlying population and are prone to varying case-definitions and diagnostic methods. Finally, hospital discharge data for the relative differences in casefatality for respiratory syncytial virus and influenza compared with pneumococcus and $H$ influenzae B were limited to high-income and middle-income countries. Several of these shortcomings are being addressed by the Pneumonia Etiological Research for Child Health project. ${ }^{110}$

\section{Injuries}

Most global road traffic deaths occur in low-income and middle-income countries and are rapidly increasing because of the growth in motorisation. Mortality rates caused by traffic-related injuries are increasing in low-income and middle-income countries. Pedestrians are most often affected, followed by car occupants and motorcyclists. Conversely, traffic deaths are decreasing in high-income countries. We noted a similar divergence between low-income and high-income countries for occupational injuries: they generally fell in high-income countries (with the exception of deaths resulting from asbestos-related mesotheliomas), whereas occupational injury deaths have increased in low-income countries (data not shown).

Suicide continues to be a major public health problem in many regions. Half of all suicide deaths occur in China and India alone. However, the trends are in opposite directions, decreasing rapidly in China but rising in India between 1990 and 2013. Both countries have undergone economic growth and urbanisation, a key factor in limiting access to lethal pesticides, a common method of suicide by poisoning in both countries. ${ }^{111}$ Therefore, as yet unexplained reasons must exist for the divergence between the two countries.

We recorded several sharp increases in mortality caused by war and disaster. Particularly, the 2010 Haiti earthquake, conflict in Syria over the past several years, the 2011 Tōhoku earthquake and tsunami in Japan, and conflict in Libya in 2011 have caused considerable loss of life. The war in Syria led to an estimated 29947 deaths (19392-54903) in 2013, and about 10504 deaths and 21422 deaths in each of the preceding 2 years. Uncertainty around these estimates is large because several different estimates exist. These estimates are of the direct deaths attributable to armed conflicts and natural disasters and do not account for the full effects of mechanisms such as the breakdown of health systems or critical infrastructure. For example, the conflict in Syria has had a substantial effect on routine immunisation for polio, with coverage now as low as $50 \%$ in some areas. ${ }^{112}$ The estimation of direct deaths caused by war and natural disasters is one of the most challenging components of the GBD measurement. We depend on the work of various groups to collate combatant reports, newspaper reports, humanitarian agency assessments, and other direct accounts to approximate the number of deaths. Vital registration systems often do not function in war or conflict but might be more useful in countries with natural disasters as a way of measuring the number of deaths. More work is needed to better measure shock mortality.

\section{India}

India accounts for 19\% of the world's deaths in 2013 . Estimations of cause of death for India are important both for health policy in India and for global understanding of causes of death. India has had remarkable progress in reducing both child and adult mortality over the past 23 years. Average yearly rates of decline were $1.3 \%$ per year for adults and $3 \cdot 7 \%$ for children.

Unfortunately, less cause of death data were available for 2013 than for 1990 or 2000. The Medical Certification of Causes of Death system provided ongoing information about patterns of urban mortality with better completeness in some states than in others. In rural areas, the Survey of Causes of Death (Rural) routinely reported causes of death from verbal autopsy from 1980 to 1998. This survey was replaced with a verbal autopsy sample collected by the Registrar-General of India based on the ongoing Sample Registration Scheme. Data for 2002-04 have been reported but not in full detail-results were released in a series of articles spanning 2008-14 but even these have not provided the standard tabulation of deaths by International Classification of Diseases cause, age, and sex used by most countries. Verbal autopsies were collected after 2004 but no data have been analysed or released. Attempts to 
add verbal autopsy to other major data collection efforts of the Government of India, such as the Annual Health Survey and the latest round of the District Level Household Survey, have so far been unsuccessful. Small community studies continue to be published but there is a major gap in knowledge of rural cause of death.

In view of the rapid change in India, including decreases in child mortality and adult mortality, simple predictions based on the 2002-04 data are inadequate. Our modelling strategy takes into account trends for key covariates that explain some changes in age-specific rates for many causes; nevertheless, more recent national data would be helpful to develop more precise estimates of causes of death for India. Epidemics such as Chikungunya, dengue, and H1N1 influenza also point to the need for better ongoing surveillance of causes of death in India that does not suffer from long time lags. ${ }^{113-116}$

\section{Comparing different global health estimates}

Comparison of the GBD 2013 results with GBD 2010 for 1990 or 2010 shows some important differences. The overall correlation coefficient of age-sex-country-cause rates was 0.998 in both 1990 and 2010 but some causes have changed substantially at the global level. The ten causes in terms of the largest change in the number of global deaths were Alzheimer's disease and other dementias, ischaemic heart disease, interstitial lung disease and pulmonary sarcoidosis, cerebrovascular disease, neonatal encephalopathy caused by birth asphyxia and trauma, lower respiratory infections, other cardiovascular and circulatory diseases, cirrhosis, malaria, and chronic kidney disease. These changes might be because of new data, modifications of garbage coding algorithms, and revised modelling strategies (appendix). Generally, the data used has substantially increased: from 8967 site-years to 14244 site-years.

Some specific changes are worth noting. First, data for China has greatly increased. Given China's population, the incorporation of large amounts of new data for cause of death led to large changes in China and these affected even global estimates. The five largest changes for China in 2013 compared with the GBD 2010 were ischaemic heart disease, Alzheimer's disease and other dementias, cerebrovascular disease, interstitial lung disease and pulmonary sarcoidosis, and chronic obstructive pulmonary disease. Second, more detailed cause of death data covering 189 causes instead of 98 causes were available for Russia for the GBD 2013. This affected several smaller causes, such as those related to alcohol. Third, we included new vital registration data for Turkey for 2010-12. Fourth, we modelled India in two components, urban and rural, which enabled us to make much more use of some data sources such as the Survey of Causes of Death (Rural) for rural India. Because India is large, these changes have a global effect. Fifth, for cancers, we incorporated 1145 registry-years of new data, including 128 from the Cancer Incidence in Five Continents Volume X ${ }^{48}$ Sixth, the change to use of a Bayesian noise reduction algorithm for smoothing has reduced the number of outliers, particularly in small verbal autopsy studies, some of which were included in the GBD 2010. Seventh, changes to garbage code redistribution algorithms, particularly the use of statistically derived algorithms that vary by region and country, has had effects on injuries, cancers, and cardiovascular diseases. Other changes included treating unspecified anaemia as a garbage code whereas in the GBD 2010 it was mapped to iron-deficiency anaemia, moving abdominal hernia from other digestive diseases to hernia, as well as moving deaths related to specific procedures to the category of adverse effects of medical treatment. In the GBD 2010, we included abdominal hernia, including umbilical hernia, ventral hernia, and diaphragmatic hernia in the category "other digestive diseases". In the GBD 2013, we combined these with inguinal hernia and femoral hernia into one cause named "hernia". Additionally, we moved some ill-defined causes from the other digestive diseases category to more specific causes, thereby reducing the number of deaths in other digestive and changing the distribution of all digestive deaths among its more disaggregated causes. Seventh, the assessment of all-cause mortality in the GBD 2013 benefited from both new data and improved approaches for assessment of the age pattern of mortality in the model life-table system. Finally, the more detailed analysis of HIV/AIDS led to major changes both for HIV/AIDS (particularly in countries with concentrated epidemics) and for other causes, particularly in the people of reproductive age and in countries with moderate-to-large epidemics.

The International Agency for Research on Cancer produces cancer estimates by country, age, sex, and cancer site for 2008 and 2012 (GLOBOCAN). Our definitions and the GLOBOCAN definitions are compatible for 25 sites. For these cancer sites, the total estimated prevalence from GLOBOCAN was 6848204 cases in 2008 and 7483018 cases in 2012. By comparison, the GBD estimates were 6930377 for 2008, and 7437018 for 2012. Worldwide, the largest variation in estimates occurs for thyroid cancer, testicular cancer, and other pharynx cancers, with differences of $20-30 \%$. The rough similarity of results at worldwide masks substantial national variation. Comparing age-standardised death rates for 2012, the correlation ranges from 0.94 for tracheal, bronchus, and lung cancer, to $0 \cdot 20$ for thyroid cancer. Five cancers have correlations below $0 \cdot 5$ (ovarian, non-Hodgkin lymphoma, testicular, Hodgkin lymphoma, and thyroid). A further six cancers have correlations of 0.5-0.7 (uterine, nasopharynx, lip and oral cavity, breast, leukaemia, and multiple myeloma).

Because both GLOBOCAN and our estimates used population-based cancer registry data and vital registration data as inputs, the wide variation in results requires explanation. As with all comparisons of global health estimates, the differences stem from data, data processing, 


\begin{tabular}{|lcc|}
\hline & CHERG & GBD 2013 \\
\hline Neonates aged 0-27 days & & \\
Congenital abnormalities & $270(207-366)$ & $251(221-291)$ \\
Diarrhoea & $50(17-151)$ & $52(44-61)$ \\
Pneumonia & $325(209-470)$ & $213(186-242)$ \\
Intrapartum-related complications* & $717(610-876)$ & $657(532-770)$ \\
Sepsis or meningitis & $393(252-552)$ & $369(237-504)$ \\
Tetanus & $58(20-276)$ & $34(16-48)$ \\
Other neonatal disorders & $181(115-284)$ & $470(411-557)$ \\
All causes & $3072 \dagger$ & $2807(2719-2898)$ \\
Children aged 1-59 months & & \\
Injury & $354(274-429)$ & $350(310-394)$ \\
Diarrhoea & $751(538-1031)$ & $536(461-607)$ \\
AIDS & $159(131-185)$ & $102(95-111)$ \\
Pneumonia & $1071(977-1176)$ & $772(693-850)$ \\
Malaria & $564(432-709)$ & $699(576-855)$ \\
Measles & $114(92-176)$ & $95(52-166)$ \\
Meningitis & $180(136-237)$ & $129(98-163)$ \\
Other disorders & $1356(1112-1581)$ & $1355(1211-1524)$ \\
All causes & $4550 \dagger$ & $4039(3883-4207)$ \\
\hline
\end{tabular}

Data are thousands of deaths ( $95 \%$ uncertainty interval). GBD=Global Burden of Disease Study. CHERG=Child Health Epidemiology Reference Group. *Compares GBD cause "Neonatal encephalopathy (birth asphyxia/trauma)" with CHERG cause "intrapartum-related complications". + CHERG did not report uncertainty estimates for all-cause mortality in children.

Table 5: Comparison of GBD and CHERG estimated child deaths for select causes in 2010

For the INDEPTH Network see http:// www.indepth-network. and model development. We included a wider range of registries than did GLOBOCAN, particularly in China, and we used of a broader database of vital registration data. Our redistribution of cancer of unknown primary was based on a statistical model. The most important differences, however, probably stem from the modelling strategy. For all cancer sites in all countries, we used CODEm. GLOBOCAN used nine different methods to estimate cancer mortality depending on the country. ${ }^{83,117}$ The choice of method can lead to surprising differences in estimated rates for neighbouring countries without data. For example, the age-standardised death rate for male thyroid cancer in Timor Leste is $250 \%$ higher than that for Indonesia; age-standardised death rates for testicular cancer differ by $1300 \%$ between Mali and Mauritania. The GLOBOCAN estimates have a substantial subjective component in the choice of which modelling strategy to use and do not provide any estimate of uncertainty. Empirical assessment of the validity of the GLOBOCAN methods-for example, through cross-validation-would help to understand the strength of the approach.

Understanding causes of death begins with assessment of all-cause mortality. There are some notable differences between our assessment of global age-specific deaths and those produced by the United Nations Population Division in their World Population Prospects 2012 revision (WPP2012). For the three periods (1995-2000, 2000-05, and 2005-10) as defined in WPP2012, the total numbers of deaths were $2 \cdot 4-3 \cdot 6 \%$ higher (6.1 million-9.1 million deaths) than estimated by us. These differentials translate into a difference of 7.8 million deaths for the 5 -year period between 2005 and 2010. The difference is greatest for younger age groups. For 2005-10, estimated under-5 deaths from WPP2012 are $10 \cdot 7 \%$ higher (3.9 million more deaths) than for the GBD 2013.

The WPP2012 global under-5 death estimates were also higher than those of UNICEF; part of this difference might be a result of the agencies releasing their estimates at different times. The biggest relative difference was for the adolescent age group (age 5-14 years). For 2005-10, the estimated deaths in adolescents from WPP2012 were $45 \cdot 1 \%$ higher than in the GBD 2013, even though the absolute difference was about $2 \cdot 2$ million for a 5 -year period, less than $1 \%(2 \cdot 17$ million of $264 \cdot 7$ million $)$ of the total deaths for the same period. The differences are even greater at the GBD regional level. For 2005-10, the relative difference between WPP2012 and GBD 2013 ranged from 26.7\% (122800) lower in WPP2012 in Oceania, to $36.0 \%$ (1.9 million) higher in WPP2012 in central sub-Saharan Africa. WPP2012 tends to have high estimates of adolescent mortality compared with the GBD 2013 for all regions in sub-Saharan Africa, Andean Latin America, north Africa and Middle East, and southeast Asia. Overall, we find more differences in estimates for sub-Saharan Africa across all age group in both relative and absolute terms.

Such discrepancy originates from different assessments of child mortality rates and the difference in model life-table systems, both of which used child mortality rate to generate age-specific mortality rates. Estimating mortality for the adolescent age group is important. ${ }^{118-120}$ As part of the background research for the GBD 2013, we assessed the Demographic Health Surveys complete birth history data for age groups 5-9 years and 10-14 years and compared this data in countries with almost complete vital registration or sample registration systems, such as India. We also systematically assessed estimates of adolescent mortality from sites of the health and demographic surveillance systems, a network known as INDEPTH. When we assessed the ratio of ${ }_{5} \mathrm{q}$ (probability of death from age 5 years to age 10 years) and ${ }_{5} q_{10}$ (probability of death from age 10 years to age 15 years) to under-5 mortality, conflicting pictures arise: our GBD 2013 estimates are sometimes higher than one source and lower than the other. Further analysis is warranted to validate our approaches for estimating adolescent mortality in low-income and middle-income countries without working vital registration systems. In addition, efforts are needed to improve both data collection and method development to better estimate mortality for adolescents.

As in the GBD 2010, we noted differences for causes of child death compared with those produced by the Child Health Epidemiology Reference Group (CHERG; table 5). Given the complexity of both approaches, it is 
difficult to isolate the reason for the differences. One reason might be the different studies used: we included 5039 site-years of vital registration and 358 of verbal autopsy data compared with 578 and 192 for CHERG. Our modelling strategy was founded on modelling each individual cause separately, using the most appropriate method for each cause, and then combining the different cause estimates into an overall assessment consistent with all-cause mortality using CoDCorrect. CHERG used separate modelling strategies for HIV/AIDS, measles, pertussis, and malaria outside of Africa, and four different models for the remainder of the child causes. Separate logistic models, each with subtly incomparable cause lists, were used for neonates and children older than 1 month, for low mortality countries excluding China, for high mortality countries excluding India, for India alone, and for China alone. This partition of the world into separate models was not justified statistically-for example, they have not shown statistically different relationships with covariates for their four sets of models. Additionally, post-estimation adjustments were applied to pneumonia, meningitis, and malaria to account for intervention effectiveness; pneumonia, sepsis, meningitis, and tetanus to account for the reliance on a combined severe infection cause in the primary model; and diarrhoea, neonatal sepsis, and sudden infant death syndrome in China to account for studies that report few causes.

We used a more empirical approach. We quantified both the root-mean squared error and validity of the UIs through cross-validation; CHERG has not to date reported any cross-validation results. Given the possibility that different relationships might exist between covariates such as access to clean water or sanitation and diarrhoeal mortality in different parts of the world, we undertook a sensitivity analysis in which we excluded vital registration data from high-income regions from the models for lower respiratory infections and diarrhoea. We detected no substantial differences for estimated global cause of death patterns in these cases. Furthermore, in CHERG, neonatal causes were assumed to not cause deaths after 1 month although high quality vital registration systems routinely report deaths from these causes that extend into the second month of life.

\section{Challenges and limitations}

In the GBD 2013, we did not include several clinical pathways to death on the cause list, such as heart failure, sepsis, fungal infection, and acute kidney injury. These clinical entities following the underlying cause construct of the International Classification of Disease are treated as garbage codes and redistributed to the likely underlying cause. Although this approach is consistent with the idea of assigning each death uniquely to the underlying cause, it masks endpoints for clinical service delivery. For example, most fungal infections are relatively minor, but potentially millions of people contract invasive fungal diseases ${ }^{121}$ that can be important pathways to death. Similar assessments can be made for sepsis, acute kidney injury, and heart failure. In future iterations of the GBD, we will aim to quantify mortality that occurs through these intermediate causes. Such intermediate cause estimation cannot be presented in the same causes lists as underlying causes of death but can provide supplemental and important information that would otherwise go unrecognised in global epidemiology.

Even in high-income countries with complete vital registration systems, our results differ from official statistics. ${ }^{122}$ This difference is largely caused by the emphasis in the GBD on enhancing comparability through redistribution of deaths assigned to garbage codes. Country-specific data for cause of death show substantial national variation in coding practices. Generally, we used global or regional algorithms to redistribute deaths assigned to garbage codes. This approach is fairly coarse and does not capture local variation in certification practice or timing of implementation of coding rules. The GBD 2013 is the most detailed effort to date to try and systematically deal with garbage code redistribution. Some changes, such as the treatment of ill-defined cancers or heart failure using statistical approaches, altered the GBD 2013 results compared with the GBD 2010. We believe that the GBD results including the fraction of deaths assigned to different types of garbage codes can be useful for national statistical authorities' efforts to improve medical certification of causes of death. We also believe that through the extensive network of GBD collaborators, we can move in future research to more country-specific redistribution algorithms. To ensure comparability, however, these national variations will have to be grounded in a sound statistical approach and theory of measurement.

A study of this scope has many limitations. First is the quality of the underlying medical certification of causes of death and verbal autopsy data. Even medical certification of causes of death has limitations, which is shown by the need for garbage code redistribution. ${ }^{106,123,124}$ Moreover, verbal autopsy data vary substantially in terms of the instrument used and the training given to physicians assigning causes of death. These shortcomings might reduce the comparability of cause of death data between countries and of our estimates based on these data.

Second, we did not incorporate uncertainty from garbage code redistribution into our estimation of UIs. Propagating such uncertainty into the CODEm models will require revision of the modelling strategy or an enormous increase in computational time. As evidenced by the change for some causes compared with the GBD 2010 as a result of changes in redistribution derived from statistical methods, this is an important area for future research.

Third, the major expansion of data for China and the associated changes in the estimates for some but not all causes, shows that UIs cannot take into account data that have not been included in the analysis. 
Fourth, for some causes, CODEm produces larger UIs in high-income countries than might be expected. This difference is largely the result of heterogeneity across high-income countries for a cause that cannot be explained by the models. This effect is more notable for causes such as diabetes, for which there are reasons to believe that large variation in certification practice remains in highincome countries. ${ }^{125}$ Because diabetes or increased fasting plasma glucose is a risk factor for macrovascular outcomes, differences in how physicians interpret the meaning of underlying cause could explain such national variation in practice. In the GBD, the full consequences of high fasting plasma glucose are captured in the risk factor assessment; ${ }^{8}$ deaths caused by diabetes in our analysis were only those that were recorded on the death certificate to be the underlying cause.

Fifth, although we tried to improve the comparability of cause of death data over time through mapping variants of the International Classification of Diseases and garbage-code redistribution, some time trends might be affected by changes in diagnostic technology. Some causes, such as cancers, might have been less likely to have been diagnosed in the 1980s and 1990s, when imaging and other diagnostic techniques were not widespread.

Sixth, for chronic kidney disease, the breakdown into deaths from diabetes, hypertension, acute glomerulonephritis, and other depends on both detailed cause of death data and renal registry data. In clinical practice, assigning chronic kidney disease to a particular cause might be difficult for patients with both hypertension and diabetes.

Seventh, in some unusual cases such as chronic respiratory diseases in India, the sum of modelled estimates for CoDCorrect level 2 causes are much smaller than the level 1 modelled estimate leading to very large corrections for the CoDCorrect step. Very large corrections for CoDCorrect suggests that the component models for these causes can be improved in the future with better data or methods.

Eighth, for natural history models, most notably for HIV/AIDS, changes in parameter assumptions such as the death rate on or off antiretroviral therapy, can have a large effect on estimated mortality. We believe that progressive revision of these models improves the estimates but nevertheless, validation of natural history models is difficult. For CODEm, we were able to quantify with the cross-validation strategy model performance but this is not possible with the natural history models.

Ninth, a strength of the GBD approach is that all estimates of cause-specific mortality must sum to all-cause mortality in a country-age-sex-year group. However, this means that estimates for a specific cause are affected by the estimates for all other causes. Causes of death such as malaria, that have very wide UIs are particularly affected by the estimates of other causes.

Tenth, models used to generate estimates of all-cause mortality and cause-specific mortality make use of a long list of covariates. Uncertainty in these covariates, such as GDP per head, was not routinely quantified but nevertheless might be substantial. We were not able to propagate uncertainty in the independent variables used in the modelling stages into the final results. 95\% UIs might therefore be under-estimated. However, when we have tested in a few cases the effect of propagating uncertainty in the independent variables in the case of the HIV crude death rate, the changes to UIs, were minor (data not shown).

Eleventh, we made extraordinary efforts to propagate uncertainty throughout our all-cause mortality estimation process, which is not yet common practice in modern demographic research. However, uncertainty in covariates used in the first stage model of child and adult mortality rate was not included because of the complexity of added computation and the fact that these covariates have little effect on our final estimates, as indicated by our preliminary testing.

Lastly, empirical age patterns of mortality, which are vital for the estimation of mortality for many low-income and middle-income countries, mostly come from high-income countries with great vital registration systems and some low-income and middle-income countries in the most recent period. Countries in the sub-Saharan African region are least represented in our empirical database of age pattern of mortality (appendix pp 81-89). Propagating uncertainty from both under-5 and adult mortality rates (two key entry parameters for our new model life-table system), and from the standard life-table generation process has given our death estimates in sub-Saharan African countries substantial uncertainty; accurate documentation of age pattern of mortality in these countries are key for producing best all-cause mortality estimates in the future.

\section{Conclusion}

Global public policy to reduce premature death needs a detailed, up-to-date, and accurate understanding of progress (or lack thereof) of disease and injury control strategies. This understanding applies not just to diseases that have been the focus of global public health efforts for the past few decades, but increasingly, as we have shown, for newly recognised contributors to global health trends. Through the process of providing yearly updates, the GBD is transforming into a collective approach to global health surveillance. Ideally, it will aggregate data from all available sources and provide a coherent view of health levels and trends that is timely, valid, and local. To fully achieve a collective process of global health surveillance, the time lag will need to be shortened between data collection, reporting, and inclusion in the GBD. Public policy in countries will be much better informed if more frequent assessments are accompanied by less uncertainty around the estimates. Uncertainty will decrease not so much as a result of further methodological advances in disease 
modelling and data synthesis, but much more as a result of greater investment and awareness among countries and donors alike of the need to strengthen vital registration systems.

Global collective action to reduce mortality from major communicable diseases such as diarrhoea, measles, tetanus, tuberculosis, and, more recently, HIV/AIDS and malaria, is working, but will require continued intervention efforts and resources and will probably be even more responsive if periodic assessments such as that reported here are available and used. While progress is being made to control several major non-communicable diseases of global concern, others have been largely neglected but are rising in importance, particularly drug use disorders, cirrhosis, diabetes, and chronic kidney disease. Greater prominence to reducing disease burden from these diseases, as well as continuing priority for injury control, is strongly suggested by our analysis. The findings on global, regional, and national trends in mortality from diseases should provide an important baseline for discussions about the next generation of health goals and targets after the Millennium Development Goals.

\section{GBD 2013 Mortality and Causes of Death Collaborators}

Mohsen Naghavi, Haidong Wang, Rafael Lozano, Adrian Davis, Xiaofeng Liang, Maigeng Zhou, Stein Emil Vollset, Ayse Abbasoglu Ozgoren*, Safa Abdalla*, Foad Abd-Allah*, Muna I Abdel Aziz*, Semaw Ferede Abera*, Victor Aboyans*, Biju Abraham*, Jerry P Abraham*, Katrina E Abuabara*, Ibrahim Abubakar*, Laith J Abu-Raddad*, Niveen ME Abu-Rmeileh*, Tom Achoki*, Ademola Adelekan*, Zanfina Ademi*, Koranteng Adofo*, Arséne Kouablan Adou*, José C Adsuar*, Johan Ärnlov*,

Emilie Elisabet Agardh*, Dickens Akena*, Mazin J Al Khabouri*, Deena Alasfoor*, Mohammed Albittar*, Miguel Angel Alegretti*, Alicia V Aleman*, Zewdie Aderaw Alemu*, Rafael Alfonso-Cristancho*, Samia Alhabib*, Mohammed K Ali*, Raghib Ali*, Francois Alla*, Faris Al Lami*, Peter Allebeck*, Mohammad A AlMazroa*, Rustam Al-Shahi Salman*, Ubai Alsharif*, Elena Alvarez*, Nelson Alviz-Guzman*, Adansi A Amankwaa*, Azmeraw T Amare*, Omid Ameli*, Hassan Amini*, Walid Ammar*, H Ross Anderson*, Benjamin O Anderson*, Carl Abelardo T Antonio*, Palwasha Anwari*, Henry Apfel*, Solveig Argeseanu Cunningham*,

Valentina S Arsic Arsenijevic*, Al Artaman*, Majed Masoud Asad*, Rana J Asghar*, Reza Assadi*, Lydia S Atkins*, Charles Atkinson*, Alaa Badawi*, Maria C Bahit*, Talal Bakfalouni*,

Kalpana Balakrishnan*, Shivanthi Balalla*, Amitava Banerjee*, Ryan M Barber*, Suzanne L Barker-Collo*, Simon Barquera*,

Lars Barregard*, Lope H Barrero*, Tonatiuh Barrientos-Gutierrez*, Arindam Basu*, Sanjay Basu*, Mohammed Omar Basulaiman*, Justin Beardsley*, Neeraj Bedi*, Ettore Beghi*, Tolesa Bekele*, Michelle L Bell*, Corina Benjet*, Derrick A Bennett*, Isabela M Bensenor*, Habib Benzian*, Amelia Bertozzi-Villa*, Tariku Jibat Beyene*, Neeraj Bhala*, Ashish Bhalla*, Zulfiqar A Bhutta*, Boris Bikbov*, Aref Bin Abdulhak*, Stan Biryukov*, Jed D Blore*, Fiona M Blyth*, Megan A Bohensky*, Guilherme Borges*, Dipan Bose*, Soufiane Boufous*, Rupert R Bourne*, Lindsay N Boyers*, Michael Brainin*, Michael Brauer*, Carol E G Brayne*, Alexandra Brazinova*, Nicholas Breitborde*, Hermann Brenner*, Adam D M Briggs*, Jonathan C Brown*, Traolach S Brugha*, Geoffrey C Buckle*, Linh Ngoc Bui*, Gene Bukhman*, Michael Burch*, Ismael Ricardo Campos Nonato*, Hélène Carabin*, Rosario Cárdenas*, Jonathan Carapetis*, David O Carpenter*, Valeria Caso*, Carlos A Castañeda-Orjuela*, Ruben Estanislao Castro*, Ferrán Catalá-López*, Fiorella Cavalleri*, Jung-Chen Chang*, Fiona C Charlson*, Xuan Che*, Honglei Chen*, Yingyao Chen*,
Jian Sheng Chen*, Zhengming Chen*, Peggy Pei-Chia Chiang*, Odgerel Chimed-Ochir*, Rajiv Chowdhury*, Hanne Christensen*, Costas A Christophi*, Ting-Wu Chuang*, Sumeet S Chugh*, Massimo Cirillo*, Matthew M Coates*, Luc Edgar Coffeng*, Megan S Coggeshall*, Aaron Cohen*, Valentina Colistro*, Samantha M Colquhoun*, Mercedes Colomar*,

Leslie Trumbull Cooper*, Cyrus Cooper*, Luis M Coppola*, Monica Cortinovis*, Karen Courville*, Benjamin C Cowie*, Michael H Criqui*, John A Crump*, Lucia Cuevas-Nasu*, Iuri da Costa Leite*, Kaustubh C Dabhadkar*, Lalit Dandona*, Rakhi Dandona*, Emily Dansereau*, Paul I Dargan*, Anand Dayama*, Vanessa De la Cruz-Góngora*, Shelley F de la Vega*, Diego De Leo*, Louisa Degenhardt*, Borja del Pozo-Cruz*, Robert P Dellavalle*, Kebede Deribe*, Don C Des Jarlais*, Muluken Dessalegn*, Gabrielle A deVeber*, Samath D Dharmaratne*, Mukesh Dherani*, Jose-Luis Diaz-Ortega*, Cesar Diaz-Torne*, Daniel Dicker*, Eric L Ding*, Klara Dokova*, E Ray Dorsey*, Tim R Driscoll*, Leilei Duan*, Herbert C Duber*, Adnan M Durrani*, Beth E Ebel*, Karen M Edmond*, Richard G Ellenbogen*, Yousef Elshrek*, Sergey Petrovich Ermakov*, Holly E Erskine*, Babak Eshrati*, Alireza Esteghamati*, Kara Estep*, Thomas Fürst*, Saman Fahimi*, Anna S Fahrion*, Emerito Jose A Faraon*, Farshad Farzadfar*, Derek FJ Fay*, Andrea B Feigl*, Valery L Feigin*,

Manuela Mendonca Felicio*, Seyed-Mohammad Fereshtehnejad*, Jefferson G Fernandes*, Alize J Ferrari*, Thomas D Fleming*, Nataliya Foigt*, Kyle Foreman*, Mohammad H Forouzanfar*, F Gerry R Fowkes*, Urbano Fra Paleo*, Richard C Franklin*, Neal D Futran*, Lynne Gaffikin*, Ketevan Gambashidze*, Fortuné Gbètoho Gankpé*, Francisco Armando García-Guerra*, Ana Cristina Garcia*, Johanna M Geleijnse*, Bradford D Gessner*, Katherine B Gibney*, Richard F Gillum*, Stuart Gilmour*, Ibrahim Abdelmageem Mohamed Ginawi*, Maurice Giroud*, Elizabeth L Glaser*, Shifalika Goenka*, Hector Gomez Dantes*, Philimon Gona*, Diego Gonzalez-Medina*, Caterina Guinovart*, Rahul Gupta*, Rajeev Gupta*, Richard A Gosselin*, Carolyn C Gotay*, Atsushi Goto*, Hebe N Gouda*, Nicholas Graetz*, K Fern Greenwell*, Harish Chander Gugnani*, David Gunnell*, Reyna A Gutiérrez*, Juanita Haagsma*, Nima Hafezi-Nejad*, Holly Hagan*, Maria Hagstromer*, Yara A Halasa*, Randah Ribhi Hamadeh*, Hannah Hamavid*, Mouhanad Hammami*, Jamie Hancock*, Graeme J Hankey*, Gillian M Hansen*, Hilda L Harb*,

Heather Harewood*, Josep Maria Haro*, Rasmus Havmoeller*, Roderick J Hay*, Simon I Hay*, Mohammad T Hedayati*, Ileana B Heredia Pi*, Kyle R Heuton*, Pouria Heydarpour*, Hideki Higashi*, Martha Hijar*, Hans W Hoek*, Howard J Hoffman*, John C Hornberger*, H Dean Hosgood*, Mazeda Hossain*, Peter J Hotez*, Damian G Hoy*, Mohamed Hsairi*, Guoqing $\mathrm{Hu}^{*}$, John J Huang*, Mark D Huffman*, Andrew J Hughes*, Abdullatif Husseini*, Chantal Huynh*, Marissa Iannarone*, Kim M Iburg*, Bulat T Idrisov*, Nayu Ikeda*, Kaire Innos*, Manami Inoue*, Farhad Islami*, Samaya Ismayilova*, Kathryn H Jacobsen*, Simerjot Jassal*, Sudha P Jayaraman*, Paul N Jensen*, Vivekanand Jha*, Guohong Jiang*, Ying Jiang*, Jost B Jonas*, Jonathan Joseph*, Knud Juel*,

Edmond Kato Kabagambe*, Haidong Kan*, André Karch*, Chante Karimkhani*, Ganesan Karthikeyan*, Nicholas Kassebaum*, Anil Kaul*, Norito Kawakami*, Konstantin Kazanjan*, Dhruv S Kazi*, Andrew H Kemp*, Andre Pascal Kengne*, Andre Keren*, Maia Kereselidze*, Yousef Saleh Khader*,

Shams Eldin Ali Hassan Khalifa*, Ejaz Ahmed Khan*, Gulfaraz Khan*, Young-Ho Khang*, Christian Kieling*, Yohannes Kinfu*,

Jonas M Kinge*, Daniel Kim*, Sungroul Kim*, Miia Kivipelto*, Luke Knibbs*, Ann Kristin Knudsen*, Yoshihiro Kokubo*, Sowarta Kosen*, Meera Kotagal*, Michael A Kravchenko*, Sanjay Krishnaswami*, Hans Krueger*, Barthelemy Kuate Defo*, Ernst J Kuipers*, Burcu Kucuk Bicer*, Chanda Kulkarni*,

Veena S Kulkarni*, Kaushalendra Kumar*, Ravi B Kumar*, Gene F Kwan*, Hmwe Kyu*, Taavi Lai*, Arjun Lakshmana Balaji*, Ratilal Lalloo*, Tea Lallukka*, Hilton Lam*, Qing Lan*,

Van C Lansingh*, Heidi J Larson*, Anders Larsson*, Pablo M Lavados*, Alicia EB Lawrynowicz*, Janet L Leasher*, Jong-Tae Lee*, James Leigh*, 
Mall Leinsalu*, Ricky Leung*, Carly Levitz*, Bin Li*, Yichong Li*, Yongmei Li*, Chelsea Liddell*, Stephen S Lim*, Graça Maria Ferreira de Lima*, Maggie L Lind*, Steven E Lipshultz*, Shiwei Liu*, Yang Liu*, Belinda K Lloyd*, Katherine T Lofgren*, Giancarlo Logroscino*, Stephanie J London*, Joannie Lortet-Tieulent*, Paulo A Lotufo*, Robyn M Lucas*, Raimundas Lunevicius* Ronan Anthony Lyons*, Stefan Ma*, Vasco Manuel Pedro Machado*, Michael F MacIntyre*, Mark T Mackay*, Jennifer H MacLachlan*, Carlos Magis-Rodriguez*, Abbas A Mahdi*, Marek Majdan*, Reza Malekzadeh*, Srikanth Mangalam*, Christopher Chabila Mapoma*, Marape Marape*, Wagner Marcenes*, Christopher Margono*, Guy B Marks*, Melvin Barrientos Marzan*, Joseph R Masci*, Mohammad Taufiq Mashal*, Felix Masiye*, Amanda J Mason-Jones*, Richard Matzopolous*, Bongani M Mayosi*, Tasara T Mazorodze*, John J McGrath*, Abigail C McKay*, Martin McKee*, Abigail McLain*, Peter A Meaney*, Man Mohan Mehndiratta*, Fabiola Mejia-Rodriguez*, Yohannes Adama Melaku*, Michele Meltzer*, Ziad A Memish*, Walter Mendoza*, George A Mensah*, Atte Meretoja*, Francis A Mhimbira*, Ted R Miller*, Edward J Mills*, Awoke Misganaw*, Santosh K Mishra*, Charles N Mock*, Terrie E Moffitt*, Norlinah Mohamed Ibrahim*, Karzan Abdulmuhsin Mohammad*, Ali H Mokdad*, Glen Liddell Mola*, Lorenzo Monasta*, Jonathan de la Cruz Monis*, Julio C Montañez Hernandez*, Marcella Montico*, Thomas J Montine*, Meghan D Mooney*, Ami R Moore*, Maziar Moradi-Lakeh*, Andrew E Moran*, Rintaro Mori*, Joanna Moschandreas*, Wilkister Nyaora Moturi*, Madeline L Moyer*, Dariush Mozaffarian*, Ulrich O Mueller*, Mitsuru Mukaigawara*, Erin C Mullany*, Joseph Murray*, Adetoun Mustapha*, Paria Naghavi*, Aliya Naheed*, Kovin S Naidoo*, Luigi Naldi*, Devina Nand*, Vinay Nangia*, KM Venkat Narayan*, Denis Nash*, Jamal Nasher*, Chakib Nejjari*, Robert G Nelson*, Marian Neuhouser*, Sudan Prasad Neupane*, Polly A Newcomb*, Lori Newman*, Charles R Newton*, Marie Ng*, Frida Namnyak Ngalesoni*, Grant Nguyen*, Nhung thi Trang Nguyen*, Muhammad Imran Nisar*, Sandra Nolte*, Ole F Norheim*, Rosana E Norman*, Bo Norrving*, Luke Nyakarahuka*, Shaun Odell*, Martin O’Donnell*, Takayoshi Ohkubo*, Summer Lockett Ohno*, Bolajoko O Olusanya*, Saad B Omer*, John Nelson Opio*, Orish Ebere Orisakwe*, Katrina F Ortblad*, Alberto Ortiz*, Maria Lourdes K Otayza*, Amanda W Pain*, Jeyaraj D Pandian*, Carlo Irwin Panelo*, Jeemon Panniyammakal*, Christina Papachristou*, Angel J Paternina Caicedo*, Scott B Patten*, George C Patton*, Vinod K Paul*, Boris Pavlin*, Neil Pearce*, Carlos A Pellegrini*, David M Pereira*, Sophie C Peresson*, Rogelio Perez-Padilla*, Fernando P Perez-Ruiz*, Norberto Perico*, Aslam Pervaiz*, Konrad Pesudovs*, Carrie B Peterson*, Max Petzold*, Bryan K Phillips*, David E Phillips*, Michael R Phillips*, Dietrich Plass*, Frédéric Bernard Piel*, Dan Poenaru*, Suzanne Polinder*, Svetlana Popova*, Richie G Poulton*, Farshad Pourmalek*, Dorairaj Prabhakaran*, Dima Qato*, Amado D Quezada*, D Alex Quistberg*, Felicia Rabito*, Anwar Rafay*, Kazem Rahimi*, Vafa Rahimi-Movaghar*, Sajjad UR Rahman*, Murugesan Raju*, Ivo Rakovac*, Saleem M Rana*, Amany Refaat*, Giuseppe Remuzzi*, Antonio L Ribeiro*, Stefano Ricci*, Patricia M Riccio*, Lee Richardson*, Jan Hendrik Richardus*, Bayard Roberts*, D Allen Roberts*, Margaret Robinson*, Anna Roca*, Alina Rodriguez*, David Rojas-Rueda*, Luca Ronfani*, Robin Room*, Gregory A Roth*, Dietrich Rothenbacher*, David H Rothstein*, Jane TF Rowley*, Nobhojit Roy*, George M Ruhago*, Lesley Rushton*, Sankar Sambandam*, Kjetil Søreide*, Mohammad Yahya Saeedi*, Sukanta Saha*, Ramesh Sahathevan*, Mohammad Ali Sahraian*, Berhe Weldearegawi Sahle*, Joshua A Salomon*, Deborah Salvo*, Genesis May J Samonte*, Uchechukwu Sampson*, Juan Ramon Sanabria*, Logan Sandar*, Itamar S Santos*, Maheswar Satpathy*, Monika Sawhney*, Mete Saylan*, Peter Scarborough*, Ben Schöttker*, Jürgen C Schmidt*, Ione JC Schneider*, Austin E Schumacher*, David C Schwebel*, James G Scott*, Sadaf G Sepanlou*, Edson E Servan-Mori*, Katya Shackelford*, Amira Shaheen*, Saeid Shahraz*, Marina Shakh-Nazarova*, Siyi Shangguan*, Jun She*,
Sara Sheikhbahaei*, Donald S Shepard*, Kenji Shibuya*,

Yukito Shinohara*, Kawkab Shishani*, Ivy Shiue*, Rupak Shivakoti*, Mark G Shrime*, Inga Dora Sigfusdottir*, Donald H Silberberg*, Andrea P Silva*, Edgar P Simard*, Shireen Sindi*, Jasvinder A Singh*, Lavanya Singh*, Edgar Sioson*, Vegard Skirbekk*, Karen Sliwa*, Samuel So*, Michael Soljak*, Samir Soneji*, Sergey S Soshnikov*, Luciano A Sposato*, Chandrashekhar T Sreeramareddy*, Jeffrey D Stanaway*, Vasiliki Kalliopi Stathopoulou*, Kyle Steenland*, Claudia Stein*, Caitlyn Steiner*, Antony Stevens*, Heidi Stöckl*, Kurt Straif*, Konstantinos Stroumpoulis*, Lela Sturua*, Bruno F Sunguya*, Soumya Swaminathan*, Mamta Swaroop*, Bryan L Sykes*, Karen M Tabb*, Ken Takahashi*, Roberto Tchio Talongwa*, Feng Tan*, David Tanne*, Marcel Tanner*, Mohammad Tavakkoli*, Braden Te Ao*, Carolina Maria Teixeira*, Tara Templin*, Eric Yeboah Tenkorang*, Abdullah Sulieman Terkawi*, Bernadette A Thomas*, Andrew L Thorne-Lyman*, Amanda G Thrift*, George D Thurston*, Taavi Tillmann*, David L Tirschwell* Imad M Tleyjeh*, Marcello Tonelli*, Fotis Topouzis*, Jeffrey A Towbin*, Hideaki Toyoshima*, Jefferson Traebert*, Bach X Tran*, Thomas Truelsen*, Ulises Trujillo*, Matias Trillini*, Zacharie Tsala Dimbuene*, Miltiadis Tsilimbaris*, E Murat Tuzcu*, Clotilde Ubeda*, Uche S Uchendu*, Kingsley N Ukwaja*, Eduardo A Undurraga*, Andrew J Vallely*, Steven van de Vijver*, Coen H van Gool*, Yuri Y Varakin*, Tommi J Vasankari*, Ana Maria Nogales Vasconcelos*, Monica S Vavilala*, N Venketasubramanian*, Lakshmi Vijayakumar*, Salvador Villalpando*, Francesco S Violante*, Vasiliy Victorovich Vlassov*, Gregory R Wagner*, Stephen G Waller*, JianLi Wang*, Linhong Wang*, XiaoRong Wang*, Yanping Wang*, Tati Suryati Warouw*, Scott Weichenthal*, Elisabete Weiderpass*, Robert G Weintraub*, Wang Wenzhi*, Andrea Werdecker*, K Ryan R Wessells*, Ronny Westerman*, Harvey A Whiteford*, James D Wilkinson*, Thomas Neil Williams*, Solomon Meseret Woldeyohannes*, Charles DA Wolfe*, Timothy M Wolock*, Anthony D Woolf*, John Q Wong*, Jonathan L Wright*, Sarah Wulf*, Brittany Wurtz*, Gelin Xu*, Yang C Yang*, Yuichiro Yano*, Hiroshi Yatsuya*, Paul Yip*, Naohiro Yonemoto*, Seok-Jun Yoon*, Mustafa Younis*, Chuanhua Yu*, Kim Yun Jin*, Maysaa El Sayed Zaki*,

Mohammed Fouad Zamakhshary*, Hajo Zeeb*, Yong Zhang*, Yong Zhao*, Yingfeng Zheng*, Jun Zhu*, Shankuan Zhu*, David Zonies*, Xiao Nong Zou*, Joseph R Zunt*, Theo Vost, Alan D Lopez†, Christopher JL Murray†. *Authors listed alphabetically. $\dagger$ †oint senior authors.

\section{Affiliations}

Institute for Health Metrics and Evaluation (M Naghavi PhD, Wang H PhD, R Lozano PhD, S E Vollset MD, T Achoki MD, H Apfel BA, C Atkinson BS, R M Barber BS, A Bertozzi-Villa BS, S Biryukov BS, J C Brown MAIS, M M Coates BS, L E Coffeng PhD, M S Coggeshall BA, Prof L Dandona PhD, E Dansereau BA, D Dicker BS, H C Duber MD, K Estep MPA, T D Fleming BS, M H Forouzanfar PhD, D Gonzalez-Medina BA, N Graetz BS, J Haagsma PhD, H Hamavid BA, J Hancock MLS, G M Hansen MSW, K R Heuton BS, H Higashi PhD, C Huynh BA, M Iannarone MSc, J Joseph BS, N Kassebaum MD, H H Kyu PhD, C Levitz MPH, C Liddell BE, M L Lind BS, K T Lofgren MPH, M F MacIntyre MEd, C Margono BS, A McLain MA, A H Mokdad PhD, M D Mooney BS, M Moradi-Lakeh PhD, M Moyer BA, E C Mullany BA, P Naghavi BESc, M Ng PhD, G Nguyen BA, S Odell MPP, S L Ohno BA, K F Ortblad MPH, A W Pain MPH, D E Phillips BS, B K Phillips BA, L Richardson BS, D A Roberts BS, M Robinson BA, G A Roth MD, L Sandar BS, A E Schumacher BS, K Shackelford BA, L Singh BS, E Sioson MS, J Stanaway PhD, C Steiner MPH, A Stevens PhD, T Templin BA, B A Thomas MD, T M Wolock BA, S Wulf MPH, B Wurtz MPH, T Vos PhD, Prof A D Lopez PhD,

Prof C J L Murray DPhil), School of Medicine (Prof R G Ellenbogen MD, J L Wright MD), Children's Hospital (N Kassebaum MD), Department of Neurology (D L Tirschwell MD), Harborview Injury Prevention and Research Center (B E Ebel MD), University of Washington, Seattle, WA, USA (R Alfonso-Cristancho PhD, Prof B O Anderson MD,

Prof N D Futran MD, P N Jensen MPH, M Kotagal MD Prof C N Mock PhD, T J Montine PhD, Prof C A Pellegrini MD, 
D A Quistberg PhD, M S Vavilala MD, Prof J R Zunt MD); National Institute of Public Health Mexico, Mexico City, Mexico (R Lozano, S Barquera PhD, T Barrientos-Gutierrez PhD, I R Campos Nonato PhD, Prof L Cuevas-Nasu MsC, V De la Cruz-Góngora MsC J-L Diaz-Ortega MD, Prof F A García-Guerra MSc, H Gomez Dantes MC, I B Heredia Pi PhD, F Mejia-Rodriguez MD, J C Montañez Hernandez MsC, Prof R Perez-Padilla MD A D Quezada MSc, D Salvo PhD, Prof E E Servan-Mori MSc S Villalpando PhD); Public Health England, London (Prof A Davis PhD, D F J Fay MSc, J C Schmidt Dott.med.), Oxford, UK (A J Hughes MSc); National Center for Chronic and Noncommunicable Disease Control and Prevention (Prof M Zhou PhD, L Duan MD, Y Li MPH, S Liu PhD, Prof L Wang MD), National Institute of Occupational Health and Poison Control (Prof F Tan MD), China Centers for Disease Control and Prevention, Beijing, China (Prof X Liang PhD); Norwegian Institute of Public Health, Bergen (S E Vollset, A K Knudsen PhD), Oslo, Norway (J M Kinge PhD, Prof V Skirbekk PhD); University of Bergen, Bergen, Norway (S E Vollset, Prof O F Norheim PhD); Hacettepe University Institute of Population Studies, Ankara, Turkey

(A Abbasoglu Ozgoren MA, B Kucuk Bicer MD); Sudanese Public Health Consultancy Group, Solihull, UK (S Abdalla MD); Faculty of Medicine,Cairo-University, Cairo, Egypt (Prof F Abd-Allah MD); Public Health Institute, Khartoum, Sudan (M I Abdel Aziz PhD); School of Public Health, College of Health Sciences (S F Abera MSc), Mekelle University, Mekelle, Tigray, Ethiopia (Y A Melaku MPH, B W Sahle MSc); Dupuytren University Hospital, Limoges, Limoges, France (Prof V Aboyans PhD); Oslo and Akershus University College of Applied Sciences (HiOA), Oslo, Norway (B Abraham M.Phil); University of Texas School of Medicine San Antonio, San Antonio, TX, USA (J P Abraham MD); University of Pennsylvania, Philadelphia, PA, USA (K E Abuabara MD, Prof D H Silberberg MD); Department of Epidemiology and Public Health (H Benzian PhD), University College London, London, UK (H Benzian PhD); Weill Cornell Medical CollegeQatar, Doha, Qatar (L J Abu-Raddad PhD); Institute of Community and Public Health, Birzeti University, Ramallah, West Bank, Occupied Palestinian Territory (N M E Abu-Rmeileh PhD); Public Health Promotion Alliance, Osogbp, Nigeria (A Adelekan PHD); Beneral Practice and Primary Health Care Academic Centre (P P-C Chiang PhD), Centre for International Child Health (S M Colquhoun $\mathrm{PhD}$ ), University of Melbourne, Melbourne, VIC, Australia (Z Ademi PhD, J D Blore PhD, M A Bohensky PhD, A Lakshmana Balaji MBBS, Prof G C Patton MD R G Weintraub MB, Prof A D Lopez PhD); Kwame Nkrumah University of Science and Technology, Kumasi, Ashanti, Ghana (K Adofo MPH); Association Ivoirienne pour le Bien-Être Familial, Abidjan, Côte d'Ivoire Côte d'Ivoire (A K Adou MD); University of Extremadura, Cáceres, Spain (Prof J C Adsuar PhD, U Fra.Paleo PhD); Uppsala University, Uppsala, Sweden (J Ärnlöv PhD, Prof A Larsson PhD); Institution of Public Health Sciences, Stockholm, Sweden (E E Agardh PhD); Makerere University, Kampala, Uganda (D Akena PhD, L Nyakarahuka MPH); Ministry of Health, Muscat, Oman (M J Al Khabouri PhD, D Alasfoor MSc); Baghdad College of Medicine, Baghdad, Baghdad, Iraq (F H Al Lami PhD); Independent, Damascus, Syria (M I Albittar BS); Grupo de Investigación en Ciencias de la Salud y Neurociencias (CISNEURO), Cartagena de Indias, Bolívar, Colombia (G Alcalá-Cerra MD); Universidad de la República, Facultad de Medicina, Departamento de Medicina Preventiva y Social, Montevideo, Uruguay (M A Alegretti MD, A V Aleman MD, F Cavalleri BS, V Colistro MSc); Debre Markos University, Debre Markos, Amhara, Ethiopia (Z A Alemu MPH); National Guard Health Affairs, Riyadh, Kingdom of Saudi Arabia (S Alhabib PhD); Rollins School of Public Health (E P Simard PhD, Prof K Steenland PhD), Emory University, Atlanta, GA, USA (M K Ali MBChB, S Argeseanu Cunningham PhD, K C Dabhadkar MPH, Prof Y Liu PhD, Prof K M V Narayan MD, S B Omer PhD, Prof M R Phillips MD); University of Oxford, Oxford, UK (R Ali MSc, D A Bennett PhD, A D M Briggs MSc,

Prof Z Chen PhD, Prof S I Hay DSc, F B Piel PhD, K Rahimi DM, P Scarborough DPhil); School of Public Health, University of Lorraine, Nancy, France (Prof F Alla PhD); Department of Public Health Sciences (Prof P Allebeck PhD), Department of Neurobiology, Care Sciences, and Society (NVS) (S-M Fereshtehnejad MD), Aging Research Center (Prof M Kivipelto PhD), Department of Medical Epidemiology and
Biostatistics, Stockholm, Sweden (Prof E Weiderpass PhD), Karolinska Institutet, Stockholm, Sweden (M Hagstromer PhD, R Havmoeller PhD S Sindi PhD); Saudi Ministry of Health, Riyadh, Kingdom of Saudi Arabia (M A A AlMazroa MD, M O Basulaiman PhD,

Prof Z A Memish MD, M Y Saeedi PhD); University of Edinburgh, Edinburgh, UK (Prof R Al-Shahi Salman PhD, Prof F G R Fowkes PhD); Charité-Universitätsmedizin Berlin, Berlin, Germany (U Alsharif DMD, S Nolte PhD, C Papachristou PhD); Government, Madrid, Spain (E Alvarez PhD); Universidad de Cartagena, Cartagena de Indias, Colombia (Prof N Alviz-Guzman PhD, A J Paternina Caicedo MSc); Albany State University, Albany, GA, USA

(Prof A A Amankwaa PhD); Department of Epidemiology, University of Groningen, Groningen, The Netherlands (A T Amare MPH); College of Medicine and Health Sciences, Bahir Dar University, Bahir Dar, Ethiopia (A T Amare); Boston University, Boston, MA, USA (O Ameli MD); Kurdistan Environmental Health Research Center, Kurdistan University of Medical Sciences, Sanandaj, Kurdistan, Iran (H Amini MSPH); Department of Epidemiology and Public Health (H Amini), Swiss Tropical and Public Health Institute, Basel, Switzerland; University of Basel, Basel, Switzerland (Prof M Tanner PhD); Ministry of Public Health, Beirut, Lebanon (Prof W Ammar PhD, H L Harb MPH); St George's, University of London, London, UK (Prof H R Anderson MD); College of Public Health, University of the Philippines Manila, Manila, Philippines (C A T Antonio MD, E J A Faraon MD, C I A Panelo MA); UNFPA, Kabul, Afghanistan (P Anwari MSc); University of Belgrade, School of Medicine, Institute of Microbiology and Immunology, Belgrade, Serbia

(Prof V S Arsic Arsenijevic PhD); Evidera Inc, Lexington, MA, USA (A Artaman PhD); Ministry of Health, Amman, Jordan (M M Asad PhD); South Asian Public Health Forum, Islamabad, Pakistan

(R J Asghar MD); Mashhad University of Medical Sciences, Mashhad, Khorasan Razavi, Iran (R Assadi MD); Ministry Of Health, Wellness, Human Services and Gender Relations, Castries, St. Lucia (L S Atkins MPH); Public Health Agency of Canada, Toronto, ON, Canada (A Badawi PhD); INECO Neurociencias, Rosario, Santa Fe, Argentina (M C Bahit MD); Ministry of Health, Damascus, Syria (T Bakfalouni MD); Sri Ramachandra University, Chennai, Tamil Nadu, India (Prof K Balakrishnan PhD, S Sambandam PhD); National Institute for Stroke and Applied Neurosciences (S Balala MPH,

Prof V L Feigin PhD), School of Psychology (S L Barker-Collo PhD), University of Auckland, Auckland, New Zealand (B del Pozo-Cruz PhD); University of Birmingham, Birmingham, UK (A Banerjee MA); Department of Occupational and Environmental Health, University of Gothenburg, Gothenburg, Sweden (Prof L Barregard PhD); Department of Industrial Engineering, Pontificia Universidad Javeriana, Bogota, Colombia (L H Barrero ScD); School of Health Sciences, University of Canterbury, Christchurch, Canterbury, New Zealand (A Basu PhD); Asian Liver Center, Palo Alto (Prof S So MBBS), School of Medicine, Stanford (L Gaffikin DrPH), Stanford University, Stanford, CA, USA (S Basu PhD); Oxford University, Ho Hi Minh City, Vietnam (J Beardsley MBChB); College of Public Health and Tropical Medicine, Jazan, Kingdom of Saudi Arabia (Prof N Bedi MD); IRCCS-Istituto di Ricerche Farmacologiche Mario Negri, Milano (E Beghi MD), Bergamo, Italy (M Cortinovis BiotechD, N Perico MD, Prof G Remuzzi MD, M Trillini MD); Madawalabu University, Ethiopia, Bale Goba, Oromia, Ethiopia (T Bekele MPH); Yale University, New Haven, CT, USA (Prof M L Bell PhD, J J Huang MD); National Institute of Psychiatry Ramon de la Fuente, Mexico City, DF, Mexico (C Benjet PhD, R A Gutiérrez PhD); Hospital Universitário (I M Bensenor PhD, Prof A H Kemp PhD), University of Sao Paulo, Sao Paulo, Brazil (Prof P A Lotufo DrPH, Prof I S Santos PhD); Addis Ababa University, Debre Zeit, Ethiopia (T J Beyene DVM, K Deribe MPH); Wellington Hospital, Wellington, New Zealand (N Bhala Dphil); Post Graduate Institute of Medical Education and Research, Chandigarh, UT, India (Prof A Bhalla MD); Medical Center (Z A Bhutta PhD), Aga Khan University, Karachi, Pakistan (M I Nisar MSc); I.Evdokimov Moscow State University of Medicine and Dentistry, Moscow, Russia (B Bikbov MD); University of Missouri-Kansas City, Kansas City, MO, USA (A Bin Abdulhak MD); Woolcock Institute of Medical Research (Prof G B Marks PhD), Sydney School of Public Health (T R Driscoll PhD), University of Sydney, Sydney, NSW, Australia 
(F M Blyth PhD, J Leigh PhD); Instituto Nacional de Psiquiatria, Mexico, Mexico (Prof G Borges Doctor Science); World Bank, Washington DC, DC, USA (D Bose PhD); Transport and Road Safety (TARS) Research (S Boufous PhD), The Kirby Institute (A J Vallely PhD), University of New South Wales, Sydney, NSW, Australia (Prof L Degenhardt PhD, M Satpathy PhD); Vision \& Eye Research Unit, Anglia Ruskin University, Cambridge, UK (Prof R R Bourne FRCOphth); Georgetown University School of Medicine, Washington, DC, USA (L N Boyers BA); DanubeUniversity Krems, Krems, Austria (Prof M Brainin PhD); School of Population and Public Health (H Krueger PhD), University of British Columbia, British Columbia, Canada (M Brauer PhD,

Prof C C Gotay PhD, F Pourmalek PhD); Cambridge Institute of Public Health, Cambridge, UK (Prof C E G Brayne MD); Trnava University, Faculty of Health Sciences and Social Work, Trnava, Slovakia (A Brazinova PhD, M Majdan PhD); University of Arizona, Tucson, AZ, USA (Prof N Breitborde PhD); German Cancer Research Center, Heidelberg, Germany (Prof H Brenner MD); University of Leicester, Leicester, UK (Prof T S Brugha MD); University of Massachusetts Medical School, Worcester, MA, USA (G C Buckle MPH,

Prof P Gona PhD); Hanoi School of Public Health, Hanoi, Vietnam (L N Bui MIPH, N t T Nguyen MS); Harvard Medical School (G Bukhman PhD), Harvard School of Public Health (E L Ding ScD, S Fahimi PhD, S Shangguan MD), Harvard University (A B Feigl MPH, J A Salomon PhD, M G Shrime MD); Great Ormond Street Hospital for Children, London, UK (M Burch MD); Universidad Autonoma Metropolitana, Mexico, DF, Mexico (Prof R CÃjrdenas Sc.D.); Department of Biostatistics and Epidemiology, University of Oklahoma Health Sciences Center, Oklahoma City, OK, USA (Prof H Carabin PhD); Telethon Institute for Child Health Research, Subiaco, Western Australia, Australia (Prof J R Carapetis PhD); University at Albany, Rensselaer, NY, USA (Prof D O Carpenter MD); Stroke Unit, University of Perugia, Perugia, Italy (V Caso MD); Colombian National Health Observatory, Instituto Nacional de Salud, Bogota, Colombia (C A Castañeda-Orjuela MSc); Universidad Diego Portales, Santiago, Chile (Prof R E Castro PhD); Division of Pharmacoepidemiology and Pharmacovigilance, Spanish Medicines and Healthcare Products Agency (AEMPS), Ministry of Health, Madrid, Spain (F Catalá-López PhD); National Taiwan University, Taipei, Taiwan (Prof C-C Chang PhD); School of Population Health (D G Hoy PhD), The University of Queensland Centre for Clinical Research (J G Scott PhD), University of Queensland, Brisbane (F C Charlson MPH, H E Erskine BPsySc, A J Ferrari BPsySc, H N Gouda PhD, L Knibbs PhD, Prof J J McGrath MD, Prof H A Whiteford MD), Herston, QLD, Australia (R E Norman PhD); National Institute of Health, Bethesda, MD, USA (X Che PhD); Zhongshan Hospital (J She PhD), Fudan University, Shanghai, China (Prof Y Chen PhD, Prof H Kan MD); CTSU, Nuffield Dept. of Population Health, Oxford, UK (Prof Z Chen); Division of Intramural Research, National Institute of Environmental Health Sciences, National Institutes of Health, Department of Health and Human Services, RTP, NC USA, Research Triangle Park, NC, USA (H Chen PhD, S J London MD); Institute of Bone and Joint Research, St Laonards, NSW, Australia (J S Chen PhD); Department of Environmental Epidemiology, University of Occupational and Environmental Health, Kitakyushu, Fukuoka ken, Japan (O Chimed-Ochir MPH); University of Cambridge, Cambridge, UK (R Chowdhury MD, J Murray PhD); Bispebjerg University Hospital, Copenhagen, Denmark (Prof H Christensen DMSCi); Cyprus University of Technology, Limassol, Cyprus (C A Christophi PhD); Department of Molecular Parasitology and Tropical Diseases, School of Medicine, Taipei Medical University, Taipei, Taiwan (Prof C-C Chang PhD); Center for International Tropical Medicine, College of Medicine, Taipei Medical University, Taipei, Taiwan (Prof T-W Chuang); Cedars-Sinai Medical Center, Los Angeles, CA, USA (Prof S S Chugh MD); University of Salerno, Baronissi, SA, Italy (Prof M Cirillo MD); Health Effects Institute, Boston, MA, USA (A Cohen DSc); UNICEM, Montevideo, Montevideo, Uruguay (M Colomar MSc); MRC Lifecourse Epidemiology Unit, University of Southampton, Southampton, UK (Prof C Cooper FMedSci); Mayo Clinic, Rochester, MN, USA (Prof L T Cooper MD, Prof I M Tleyjeh MD); Hospital Municipal Ramon Santamarina, Tandil, Buenos Aires, Argentina (L M Coppola MD); Victorian Infectious Diseases Reference Laboratory, North Melbourne,
Victoria, Australia (B C Cowie PhD, J H MacLachlan MSc); University of California, San Diego, La Jolla, CA, USA (Prof M H Criqui MD, Prof S K Jassal MD); Centre for International Health, Dunedin School of Medicine (Prof J A Crump MD), University of Otago, Dunedin, Otago, New Zealand (Prof R G Poulton PhD); National School of Public Health (ENSP/Fiocruz), Rio de Janeiro, Brazil (I da Costa Leite PhD); Public Health Foundation of India, New Delhi, India (Prof L Dandona, Prof R Dandona PhD, S Goenka PhD); Indian Institute of Public Health, New Delhi, Delhi (Prof R Dandona, S Goenka PhD), Gurgaon, Haryana, India (R B Kumar MD); Guy's and St Thomas' NHS Foundation Trust, London, UK (P I Dargan MBBS); Department of Surgery, Jacobi Medical Center, Atlanta, GA, USA (A Dayama MD); Institute on Aging (S F de la Vega MD), Institute of Health Policy and Development Studies, National Institutes of Health, Manila, Philippines (Prof H Lam PhD); Griffith University, Brisbane, QLD, Australia (Prof D De Leo DSc); U.S. Department of Veterans Affairs, Washington DC (U S Uchendu MD), Eastern Colorado Healthcare System, Denver, CO, USA (R P Dellavalle MD); Beth Israel Medical Center, New York City, NY, USA (Prof D C Des Jarlais PhD); Africa Medical and Research Foundation in Ethiopia, Addis Ababa, Ethiopia (M Dessalegn MPH); Hospital for Sick Children, University of Toronto, Toronto, ON, Canada (G A deVeber MD); University of Peradeniya, Peradeniya, Sri Lanka (S D Dharmaratne MD); The University of Liverpool, Liverpool, Merseyside, UK (M K Dherani PhD); Hospital de la Santa Creu i Sant Pau, Barcelona, Spain (C Diaz-Torne MD); Department of Social Medicine, Faculty of Public Health, Medical University-Varna, Varna, Bulgaria (K Dokova PhD); University of Rochester Medical Center, Rochester, NY, USA (Prof E R Dorsey MD); National Institutes of Health, Montgomery Village, MD, USA (A M Durrani MD); School of Medicine and Pharmacology (Prof G J Hankey MD), University of Western Australia, Perth, WA, Australia (Prof K M Edmond PhD); Food Science Department, Faculty of Agriculture, University of Tripoli, Tripoli, Libya (Prof Y M Elshrek PhD); The Institute of Social and Economic Studies of Population at the Russian Academy of Sciences, Moscow, Russia (Prof S P Ermakov DSc); Arak University of Medical Sciences \& Health Affairs, Arak, Markazi, Iran (B Eshrati PhD); Endocrinology and Metabolism Research Center (Prof A Esteghamati MD,N Hafezi-Nejad MD, S Sheikhbahaei MD); Non-Communicable Diseasese Reesearch Center (F Farzadfar MD), Digestive Diseases Research Center (Prof R Malekzadeh MD, S G Sepanlou MD), Sina Trauma and Surgery Research Center (Prof V Rahimi-Movaghar MD), MS Research Center (M A Sahraian MD), Department of Community Medicine (M Moradi- Lakeh), Tehran University of Medical Sciences, Tehran, Iran (P Heydarpour MD); Centre for Health Policy \& Department of Infectious Disease Epidemiology (T Fürst PhD), MRC-PHE Centre for Health and Environment (A B Mustapha PhD), Imperial College London, London, UK (K Foreman MPH, Prof A Rodriguez PhD, L Rushton PhD, M Soljak PhilD, Prof T N Williams MD); Division of Information, Evidence, Research and Innovation (C Stein PHD), World Health Organization, Regional Office for Europe, Copenhagen, Denmark (A S Fahrion Dr med vet, I Rakovac PhD), WHO, Geneva, Switzerland (L Newman MD); ARS Norte (C M Teixeira MD), I.P.Departamento Saúde Pública, Porto, Portugal (M M Felicio MD, G M F d Lima BSC, V M P Machado MSc); German Hospital Oswaldo Cruz, Institute of Education and Sciences, São Paulo, São Paulo, Brazil (Prof J G Fernandes PhD); Institute of Gerontology, Acad Med Sci, Kyiv, Ukraine (N Foigt PhD); James Cook University, Townsville, QLD, Australia (R C Franklin PhD); National Center for Disease Control \& Public Health, Tbilisi, Georgia (K Gambashidze MS, K Kazanjan MS, M Kereselidze PhD, M Shakh-Nazarova MS, L Sturua PhD); Clinique Coopérative de Parakou, Parakou, Borgou, Benin (F G Gankpé MD); Public Health Unit of Primary Health Care Group of Almada-Seixal, Almada, Setúbal, Portugal (A C Garcia MPH); Wageningen University, Division of Human Nutrition, Wageningen, Netherlands (J M Geleijnse PhD); Agence de Medecine Preventive, Paris, France (B D Gessner MD); Eastern Health Clinical School (B K Lloyd PhD), Monash University, Melbourne, VIC, Australia (K B Gibney MPH, Prof A G Thrift PHD); Howard University, Washington DC, DC, USA (Prof R F Gillum MD); Graduate School of Medicine (M Inoue PhD), School of Public Health (Prof N Kawakami MD), University of Tokyo, Tokyo, Japan (S Gilmour MPH, Prof K Shibuya DrPH); University of 
Hail, College of Medicine, Hail, Kingdom of Saudi Arabia (I A M Ginawi MD); University Hospital of Dijon, Dijon France, Region of Burgondy, France (Prof M Giroud MD); Brandeis University, Waltham, MA (E L Glaser MA, Y A Halasa MS, B T Idrisov MD, S Shahraz MD, Prof D S Shepard PhD, E A Undurraga PhD); University of California San Francisco, San Francisco, CA, USA

(R A Gosslin MD D S Kazi MD); Department of Diabetes Research, National Center for Global Health and Medicine, Tokyo, Japan (A Goto PhD); Saint James School of Medicine, Kralendijk, Bonaire, Netherlands Antilles (Prof H C Gugnani PhD); PATH, Seattle, WA, USA (C Guinovart PhD); University of Bristol, Bristol, UK

(Prof D Gunnell DSc); Fortis Escorts Hospital, Jaipur, Rajasthan, India (R Gupta MD PhD); Kanawha Charleston Health Department, Charleston, WV, USA (R Gupta MD); Nelson Institute of Environmental Medicine, New York University School of Medicine, Tuxedo (Prof G D Thurston ScD), New York University, New York, NY, USA (Prof H Hagan PhD); Arabian Gulf University, Manama, Bahrain (Prof R R Hamadeh Dphil); Wayne County Department of Health and Human Services, Detroit, MI, USA (M Hammami MD); Eunice Gibson Polyclinic, Bridgetown, St. Michael, Barbados (H C Harewood MPH); Parc Sanitari Sant Joan de Déu, CIBERSAM, University of Barcelona, Sant Boi de Llobregat, Barcelona, Spain (Prof J M Haro MD); International Foundation for Dermatology, London, UK (Prof R J Hay DM); Mazandaran University of Medical Sciences, Sari, Mazandaran, Iran (Prof M T Hedayati PhD); Fundacion Entornos AC, Cuernavaca, Morelos, Mexico (Prof M Hijar PhD); Parnassia Psychiatric Institute, The Hague, Netherlands (Prof H W Hoek MD); National Institute on Deafness and Other Communication Disorders, National Institutes of Health, Bethesda, MD, USA (H J Hoffman MA); Cedar Associates, Menlo Park, CA, USA (Prof J C Hornberger MD); Albert Einstein College of Medicine, Bronx, NY, USA (Prof H D Hosgood PhD); London School of Hygiene and Tropical Medicine, London, UK (M Hossain MSc, H J Larson PhD, Prof M McKee DSc, Prof N Pearce PhD, B Roberts PhD, H Stöckl Dphil, T Tillmann MPH); Baylor College of Medicine, Houston, TX, USA (Prof P J Hotez PhD); Public Health Division, Secretariat of the Pacific Community, Noumea, New Caledonia, Herston, Queensland, Australia (D G Hoy); National Institute of Public Health (MOH), Tunis, Tunisia (Prof M Hsairi MD); University of Toronto, Toronto, Ontario, Canada (Prof H Hu MD); Central South University, Changsha, China (Prof G Hu PhD); Feinberg School of Medicine (M D Huffman MD), Northwestern University, Chicago, IL, USA (M Swaroop MD); Public Health Program, Qatar University, Doha, Qatar, Birzeit, Ramallah, Palestine (A Husseini PhD); Aarhus University, Aarhus, Denmark (K M Iburg PhD); National Institute of Health and Nutrition, Bunkyo, Tokyo, Japan (N Ikeda PhD); National Institute for Health Development, Tallinn, Estonia (K Innos PhD, M Leinsalu PhD); American Cancer Society, New York, NY (F Islami PhD), Atlanta, GA, USA (J Lortet-Tieulent MSc); Selfemployed, Baku, Azerbaijan (S Ismayilova MPH); George Mason University, Fairfax, VA, USA (K H Jacobsen PhD); VA San Diego, San Diego, CA, USA (Prof S K Jassal); Virginia Commonwealth University, Richmond, VA, USA (S P Jayaraman MD); Postgraduate Institute of Medical Education and Research, Chandigarh, India (Prof V Jha DM); Tianjin Centers for Diseases Control and Prevention, Tianjin, China (Prof G Jiang MD); Department of Health Development, Institute of Industrial Ecological Sciences, Department of Environmental Epidemiology, University of Occupational and Environmental Health, Japan, Kitakyushu, Fukuoka, Japan (Y Jiang PhD); Department of Ophthalmology, Medical Faculty Mannheim of the University of Heidelberg, Mannheim, Germany (Prof J B Jonas MD); The National Institute of Public Health, Copenhagen, Denmark (Prof K Juel PhD); Vanderbilt University, Nashville, TN, USA (E K Kabagambe PhD, U Sampson MD); University of Balamand, Beirut, Lebanon (Prof N Karam MD); Helmholtz Centre for Infection Research, Braunschweig, Germany (A Karch MD); German Center for Infection Research (DZIF), Hannover-Braunschweig site, Braunschweig, Germany (A Karch); College of Physicians and Surgeons (C Karimkhani BA), Columbia University, New York, NY, USA (A E Moran MD); All India Institute of Medical Sciences, New Delhi, India (Prof G Karthikeyan DM, Prof V K Paul MD); Oklahoma State University, Tulsa, OK, USA (A Kaul MD); South African Medical Research Council, Cape Town,
Western Cape, South Africa (A P Kengne PhD, R Matzopolous PhD); Cardiology, Hadassah Ein Kerem University Hospital, Jerusalem, Israel (Prof A Keren MD); Jordan University of Science and Technology, AlRamtha, Irbid, Jordan (Prof Y S Khader ScD); Supreme Council of Health, Doha, Qatar (S E A H Khalifa MSc.); Health Services Academy, Islamabad, Punjab, Pakistan (E A Khan MPH); UAE University, Al Ain, Abu Dhabi, United Arab Emirates (G Khan PhD); Institute of Health Policy and Management, Seoul National University College of Medicine, Seoul, South Korea (Prof Y-H Khang MD PhD); Federal University of Rio Grande do Sul, Porto Alegre, RS, Brazil (C Kieling PhD); Northeastern University, Boston, MA, USA (Prof D Kim DrPH); Soonchunhyang University, Asan, South Korea (Prof S Kim PhD); University of Canberra, Canberra, ACT, Australia (Y Kinfu PhD); Department of Preventive Cardiology, National Cerebral and Cardiovascular Center, Suita, Osaka, Japan (Y Kokubo PhD); Center for Community Empowerment, Health Policy \& Humanities (S Kosen MD), NIHRD, Jakarta, Special Province of Jakarta, Indonesia (T S Warouw PhD); Research Center of Neurology, Moscow, Russia (M Kravchenko PhD, Prof Y Y Varakin MD); Oregon Health and Science University, Portland, OR, USA (S Krishnaswami MD); Oregon Health and Science University, Portland, OR, USA (S Krishnaswami MD); University of Montreal, Montreal, Quebec, Canada (Prof B Kuate Defo PhD); Department of Public Health (S Polinder PhD), Erasmus MC University Medical Center, Rotterdam, The Netherlands (Prof E J Kuipers PhD, Prof J H Richardus PhD); Rajrajeshwari Medical College \& Hospital, Bangalore, Karnataka, India (Prof C Kulkarni PhD); Arkansas State University, AR, USA (V S Kulkarni PhD); International Institute for Population Sciences, Mumbai, Maharashtra, India (K Kumar MPS); Boston Medical Center, Boston, MA, USA (G F Kwan MD); Fourth View Consulting, Tallinn, Estonia (T Lai PhD); Australian Research Centre for Population Oral Health (ARCPOH), Gold Coast, QLD, Australia (Prof R Lalloo PhD); School of Dentistry, The University of Adelaide, Gold Coast, QLD, Australia (Prof R Lalloo); Finnish Institute of Occupational Health, Topeliuksenkatu, Helsinki, Finland (Prof T Lallukka PhD); Medical Faculty, Hjelt Institute, University of Helsinki, Finland (Prof T Lallukka); National Cancer Institute, Rockville, MD, USA (Q Lan PhD); IAPB and Vision 2020 LA, Weston, FL, USA (V C Lansingh PhD); Servicio de Neurologí $a$, Clinica Alemana, Universidad del Desarrollo, Santiago, RM, Chile (P M Lavados MD); Instituto Nacional de Epidemiologia "Dr. Juan H Jara," Mar del Plata, Buenos Aires, Argentina (A E B Lawrynowicz MD, A P Silva MgSc, C Ubeda MPH); Nova Southeastern University, Fort Lauderdale, FL, USA (J L Leasher OD.); Korea University, Seoul, South Korea (Prof J-T Lee PhD, Prof S-J Yoon PhD); SUNY-Albany, Rensselaer, NY, USA (R Leung PhD); Department of Gerontology (Y Zhang PhD), Jinan Central Hospital, Jinan, Shandong, China (B Li PhD); Genentech, Inc, South San Francisco, CA, USA (Y Li PhD); Wayne State University, Miami, FL, USA (S E Lipshultz MD); Turning Point Alcohol \& Drug Centre, Eastern Health, Fitzroy, Victoria, Australia (B K Lloyd, R Room PhD); University of Bari, Bari, Italy (Prof G Logroscino MD PhD); The Australian National University, Canberra, ACT, Australia (Prof R M Lucas PhD); Aintree University Hospital NHS Foundation Trust, Liverpool, UK (R Lunevicius PhD); Swansea University, Swansea, UK (Prof R A Lyons MD); Ministry of Health Singapore, Singapore, Singapore (S Ma PhD); Royal Children's Hospital Melbourne, Victoria, VIC, Australia (M T Mackay MBBS); Centro para la prevención y el control del VIH /SIDA, México City, Distrito Federal, Mexico (C Magis-Rodriguez PhD); King George's Medical University, Lucknow, Uttar Pradesh, India (Prof A A Mahdi PhD); Technical Standards and Safety Authority, Toronto, Ontario, Canada (S Mangalam MS); University of Zambia, Lusaka, Zambia (C C Mapoma PhD, F Masiye PhD); Botswana-Baylor Children's Clinical Centre of Excellence, Gaborone, Botswana (M Marape PhD); Queen Mary, University of London, London, UK (Prof W Marcenes PhD); University of the East Ramon Magsaysay Medical Center, Quezon City, Metro Manila, Philippines

(M B Marzan MSc); Elmhurst Hospital Center, Mount Sinai Services, Elmhurst, NY, USA (Prof J R Masci MD); Ministry of Public Health, Kabul, Afghanistan (M T Mashal PhD); University of York, York, UK (A J Mason-Jones PhD); Faculty of Health Sciences, Hatter Institute for Cardiovascular Research in Africa (Prof K Sliwa PhD), University of 
Cape Town, Cape Town, Western Cape, South Africa

(Prof B M Mayosi Dphil); AIDC EC, Port Elizabeth, Eastern Cape, South Africa (T T Mazorodze MA); EmergentCorp, Belize City, Belize District, Belize (A C McKay PhD); Janakpuri Superspecialty Hospital, New Delhi, Delhi, India (Prof M M Mehndiratta MD); Thomas Jefferson University, Philadelphia, PA, USA (M Meltzer MD); UNFPA, Lima, Peru

(W Mendoza MD); Center for Translation Research and Implementation Science (CTRIS), National Heart, Lung, and Blood Institute, National Institutes of Health, Bethesda, MD, USA (G A Mensah); Ifakara Health Institute, Dar es Salaam, Tanzania (F A Mhimbira MSc); Pacific Institute for Research \& Evaluation, Calverton MD, USA (T R Miller PhD); Centre for Population Health Research, Curtin University, Perth, Australia (T R Miller); University of Ottawa, Ottawa, Ontario, Canada (E J Mills PhD); Population Education Resource Centre (PERC), Department of Continuing and Adult Education and Extension Work, S. N. D. T. Women's University, Mumbai, Maharashtra, India (S Mishra PhD); Duke University, Durham, NC, USA (Prof T E Moffitt PhD); Department of Medicine, Universiti Kebangsaan Malaysia Medical Center, Bandar Tun Razak, Kuala Lumpur, Malaysia (Prof N Mohamed Ibrahim MRCP); University of Salahaddin, Erbil, Iraq (K A Mohammad PhD); University of Papua New Guinea, Port Moresby, NCD, Papua New Guinea (Prof G L Mola MD); Institute for Maternal and Child Health-IRCCS “Burlo Garofolo," Trieste, Italy (L Monasta DSc, M Montico MSc, L Ronfani PhD); Bureau of International Health Cooperation, Manila City, Philippines (Prof J D L C Monis MSc Epi); University of North Texas, Denton, TX, USA (Prof A R Moore PhD); National Center for Child Health and Development, Setagaya, Tokyo, Japan (R Mori PhD); Department of Medicine, Heraklion (Prof M Tsilimbaris PhD), University of Crete, Crete, Greece (J Moschandreas PhD); Egerton University, Egerton, Rift Valley, Kenya (W N Moturi PhD); Friedman School of Nutrition Science \& Policy, Tufts University, Boston, MA, USA (D Mozaffarian DrPH); Philipps-University Marburg, Marburg, Germany

(Prof U O Mueller PhD); Tokyo Medical and Dental University, Bunkyo-ku, Tokyo, Japan (M Mukaigawara MD); International Centre for Diarrhoeal Diseases Research, Bangladesh, Dhaka, Bangladesh (A Naheed PhD); University of KwaZulu-Natal, Durban, KwaZulu-Natal, South Africa (Prof K S Naidoo PhD); Azienda Ospedaliera papa Giovanni XXIII, Bergamo, Italy, Bergamo, Italy (Prof L Naldi MD); Ministry of Health Fiji, Suva, Republic of Fiji (D Nand MPH); Suraj Eye Institute, Nagpur, Maharashtra, India (Prof V Nangia MD); School of Public Health, City University of New York, New York, NY, USA (Prof D Nash PhD); Ministry of Public Health \& Population, Sana'a, Yemen (J Nasher MSc); Faculty of Medicine, Fez, Morocco (Prof C Nejjari PhD); National Institute of Diabetes and Digestive and Kidney Diseases, Phoenix, AZ, USA (R G Nelson PhD); Fred Hutchinson Cancer Research Center, Seattle, WA, USA (M L Neuhouser PhD, P A Newcomb PhD); Norwegian Center for Addiction Research (SERAF), University of Oslo, Norway (S P Neupane Mphil); Ministry of Health and Social Welfare, Dar Es Salaam, Tanzania (F N Ngalesoni MSc); Department of Clinical Sciences, Medical Faculty, Lund University, Lund, Sweden (Prof B Norrving PhD); National University of Ireland Galway, Galway, Ireland (M J O'Donnell PhD); Teikyo University School of Medicine, Tokyo, Japan (Prof T Ohkubo MD); Center for Healthy Start Initiative, Ikoyi, Lagos, Nigeria (B O Olusanya PhD); Lira District Local Government, Lira Municipal Council, Northern Uganda, Uganda (J N OpioMPH); Toxicology Unit, Faculty of Pharmacy, University of Port Harcourt, Nigeria, Port Harcourt, Rivers State, Nigeria (Prof O E Orisakwe PhD); IIS-Fundacion Jimenez Diaz, Madrid, Spain (Prof A Ortiz PhD); Mariano Marcos Memorial Hospital \& Medical Center, City of Batac, Ilocos Norte, Philippines (M L K Otayza MD); Christian Medical College Ludhiana, Ludhiana, India (Prof J D Pandian MD); Centre for Chronic Disease Control, New Delhi, Delhi, India (J Panniyammakal PhD); University of Calgary, Calgary, Alberta, Canada (Prof S B Patten PhD, J L Wang PhD); Independent Researcher, Port Moresby, Waigani, NCD, Papua New Guinea (B I Pavlin MD); REQUIMTE/Laboratório de Farmacognosia, Departamento de Quí $\neg$ mica, Faculdade de Farmácia, Universidade do Porto, Portugal, Porto, Portugal (Prof D M Pereira PhD); International Diabetes Federation, International Diabetes Federation, Belgium
(S C Peresson MA); Hospital Universitario Cruces, Baracaldo, Spain (F P Perez-Ruiz PhD); Postgraduate Medical Institute, Lahore, Punjab, Pakistan (A Pervaiz MHA); Flinders University, Adelaide, SA, Australia (Prof K Pesudovs PhD); Aalborg University, Aalborg Øst, Denmark (C B Peterson PhD); Centre for Applied Biostatistics, Sahlgrenska Academy, University of Gothenburg, Sweden, Gothenburg, Sweden (Prof M Petzold PhD); Shanghai Jiao Tong University, Shanghai, China (Prof M R Phillips); Exposure Assessment and Environmental Health Indicators, Federal Environment Agency, Bielefeld, North RhineWestphalia, Germany (D Plass MPH); McMaster University, Hamilton, ON, Canada (Prof D Poenaru MD); Centre for Addiction and Mental Health, Toronto, Ontario, Canada (S Popova PhD); Centre for Chronic Disease Control, New Delhi, Delhi, India (Prof D Prabhakaran MD); College of Pharmacy (Prof D Qato PhD), University of Illinois, Chicago, IL, USA (K M Tabb PhD); Tulane University School of Public Health and Tropical Medicine, New Orleans, LA, USA (F Rabito PhD); Contech Intl., Lahore, Punjab, Pakistan (A Rafay MS); Hamad Medical Corporation, Doha, Qatar (S U R Rahman FCPS); University of Missouri, Columbia, MS, USA (M Raju PhD); Department of Public Health, University of the Punjab, Lahore, Pakistan, Punjab, India (S M Rana PhD); Walden University, Minneapolis, MN, USA (Prof A Refaat PhD); Hospital das Clínica da Universidade Federal de Minas Gerais, Belo Horizonte, Minas Gerais, Brazil (Prof A L Ribeiro PhD); UO Neurologia USL Umbria 1, Cittá di Castello, Perugia, Italy (S Ricci MD); Department of Clinical Neurological Sciences, London Health Sciences Centre, University of Western Ontario, London, Ontario, Canada (P M Riccio MD); MRC Unit, Fajara, The Gambia (A Roca PhD); Centre of Research in Environmental Epidemiology (CREAL), Barcelona, Catalonia, Spain

(D Rojas-Rueda PhD); Institute of Epidemiology and Medical Biometry, Ulm University, Ulm, Germany (Prof D Rothenbacher MD); Ann \& Robert H. Lurie Children's Hospital of Chicago, Chicago, IL, USA (D H Rothstein MD); BARC Hospital, Mumbai, Maharashtra, India (Prof N Roy MD); Muhimbili University of Health and Allied Sciences, Dar es Salaam, Tanzania (G M Ruhago MA, B F Sunguya MSc); Rwanda Bio-Medical Center, Kigali, Rwanda, Rwanda (N Sabin MD); Stavanger University Hospital, Stavanger, Norway (Prof K Søreide PhD); Queensland Centre for Mental Health Research, Brisbane, QLD Australia (S Saha PhD); UKM Medical Centre, Kuala Lumpur, Malaysia (R Sahathevan PhD); National HIV/AIDS \& STI Surveillance and Strategic Information Unit, National Epidemiology Center, Department of Health, Manila, National Capital Region, Philippines

(G M J Samonte MD); Case Western Reserve University, Cleveland, $\mathrm{OH}$, USA (J R Sanabria MD); Marshall University, Huntington, WV, USA (M Sawhney PhD); Novartis, Istanbul, Istanbul, Turkey (M I Saylan MD); Division of Clinical Epidemiology and Aging Research, German Cancer Research Center, Heidelberg, Baden-Württemberg, Germany (B Schöttker MPH); Federal University of Santa Catarina, Florianópolis, SC, Brazil (I J C Schneider PhD); University of Alabama at Birmingham, Birmingham, AL, USA (Prof D C Schwebel PhD, J A Singh MD); An-Najah University, Nablus, Palestine (A Shaheen PhD); Tachikawa Hospital, Tokyo, Japan (Prof Y Shinohara PhD); Washington State University, Spokane, WA, USA (K Shishani PhD); Heriot-Watt University, Edinburgh, Scotland, UK (I Shiue PhD); Center for Clinical Global Health Education (R Shivakoti PhD), Bloomberg School of Public Health, Johns Hopkins University, Baltimore, MD, USA (B X Tran PhD); Reykjavik University, Reykjavik, Iceland (Prof I D Sigfusdottir PhD); Dartmouth College, Lebanon, NH, USA (S Soneji PhD); Federal Research Institute for Health Organization and Informatics of Ministry of Health of the Russian Federation, Moscow, Russia (S S Soshnikov PhD); Department of Clinical Neurological Sciences, Western University, London, ON, Canada (L A Sposato MD); Faculty of Medicine and Health Sciences, University Tunku Abdul Rahman, Selangor, Malaysia, Kajang, Selangor, Malaysia (C T Sreeramareddy MD); Centre Hospitalier Nord Deux-Sevres, Bressuire, France (V K Stathopoulou MD); IARC/WHO, Lyon, France (K Straif MD PhD); KEELPNO, Centre for Disease Control, Greece, dispatched to "Alexandra" General Hospital of Athens, Athens, Greece (K Stroumpoulis PhD); National Institute for Research in Tuberculosis, Chennai, Tamil Nadu, India (S Swaminathan MD); Department of Criminology, Law and Society (and Sociology), University of CaliforniaIrvine, Irvine, CA, USA (B L Sykes PhD); Institute of Industrial 
Ecological Sciences, Department of Environmental Epidemiology, University of Occupational and Environmental Health, Kitakyushu, Fukuoka Prefecture, Japan (Prof K Takahashi MD); Ministry of HealthMINSANTE, Yaounde, Centre, Cameroon (R T Talongwa MD); Chaim Sheba Medical Center and Tel Aviv University, Tel Hashomer, Israel (Prof D Tanne MD); Westchester Medical Center, Valhalla, NY, USA (M Tavakkoli MD); Auckland University of Technology, Auckland, New Zealand (B J Te Ao MPH); Jhpiego, Addis Ababa, Ethiopia (A M Temesgen PhD); Memorial University, St John's, Newfoundland, Canada (E Y Tenkorang PhD); Department of Anesthesiology, University of Virginia, Charlottesville, VA, USA (A S Terkawi MD); Department of Anesthesiology, King Fahad Medical City, Riyadh, Saudi Arabia (A S Terkawi, Prof I M Tleyjeh); WorldFish, Penang, Malaysia, New York, NY, USA (A L Thorne-Lyman ScD); Alfasial University, College Of Medicine, Riyadh, Saudi Arabia (Prof I M Tleyjeh); University of Alberta, Edmonton, AB, Canada (Prof M Tonelli MD); Aristotle University of Thessaloniki, Thessaloniki, Greece (Prof F Topouzis PhD); Cincinnati Children's Hospital Medical Center, Cincinnati, OH, USA (Prof J A Towbin MD); Health Care Center of Anjo Kosei Hospital, Anjo City, Aichi Prefecture, Japan (Prof H Toyoshima MD); University of Southern Santa Catarina, Palhoça, Santa Catarina, Brazil (Prof J Traebert PhD); Department of Neurology, Copenhagen University Hospital Herlev, Herlev, Copenhagen, Denmark (T Truelsen PhD); Servicio Canario de Salud, Santa Cruz de Tenerife, Tenerife, Spain (U Trujillo MD); Department of Population Sciences and Development, Faculty of Economics and Management, University of Kinshasa, Kinshasa, Democratic Republic of the Congo (Z Tsala Dimbuene PhD); Department of Internal Medicine, Federal Teaching Hospital Abakaliki, Abakailiki, Ebonyi State, Nigeria (K N Ukwaja MD); African Population and Health Research Center, Nairobi, Kenya (S van de Vijver MD); National Institute for Public Health and the Environment, Bilthoven, The Netherlands (C H van Gool PhD); UKK Institute for Health Promotion Research, Tampere, Finland (Prof T J Vasankari MD PhD); Universidade de Brasília, Brasília, Distrito Federal-DF, Brazil (Prof A M N Vasconcelos PhD); Neuroscience Centre, Raffles Hospital, Singapore, Singapore (N Venketasubramanian MD); Voluntary Health Services, Sneha, Chennai, Tamil Nadu, India (Prof L Vijayakumar PhD); University of Bologna, Bologna, Italy (Prof F S Violante MD); Higher School of Economics, Moscow, Russia (Prof V V Vlassov MD); National Institute for Occupational Safety and Health, Washington, DC, USA (G R Wagner MD); Uniformed Services University of Health Sciences, Bethesda, MD, USA (S Waller MD); National Office for Maternal and Child's Health Surveillance, Chengdu, China (Prof Y Wang BS, Prof J Zhu MD); Health Canada, Ottawa, Ontario, Canada (S Weichenthal PhD); Murdoch Children's Research Institute, Royal Children's Hospital, Melbourne, VIC, Australia (R G Weintraub MB); Beijing Neurosurgical Institute, Beijing, China (Prof W Wenzhi MD); Institute of Medical Sociology and Social Medicine

(A Werdecker Dipl.oec.troph), Marburg, Hessen, Germany (R Westerman PhD); University of California, Davis, Davis, CA, USA (K R R Wessells PhD); University of Miami, Miami, FL, USA (J D Wilkinson MD); Institute of Public Health, University of Gondar, Gondar, Amhara, Ethiopia (S M Woldeyohannes MPH); Ateneo School of Medicine and Public Health, Pasig City, Metro Manila, Philippines (J Q Wong MD); Royal Cornwall Hospital, Truro, Cornwall, UK (Prof A D Woolf FRCP); Nanjing University School of Medicine, Jinling Hospital, Nanjing, China (Prof G Xu PhD); University of North Carolina at Chapel Hill, Chapel Hill, NC, USA (Y C Yang PhD); Division of Cardiovascular Medicine, Jichi Medical University School of Medicine, Shimotsuke, Tochigi, Japan (Y Yano MD); Fujita Health Univeristy, Toyoake, Aichi, Japan (Prof H Yatsuya PhD); The University of Hong Kong, Hong Kong, China (Prof P Yip PhD); National Center of Neurology and Psychiatry, Kodira, Tokyo, Japan (N Yonemoto MPH); Jackson State University, Jackson, MS, USA (Prof M Younis PhD); Department of Epidemiology and Biostatistics, School of Public Health, Wuhan University Global Health Institute, Wuhan University, Wuhan, Hubei, China (Prof C Yu PhD); TCM Medical TK SDN BHD, Nusajaya, Johor Bahru, Malaysia (K Yun Jin PhD); Mansoura Faculty of Medicine, Mansoura, Mansoura, Egypt (Prof M E S Zaki MD); Ministry for Planning and Training, Riyadh, Kingdom of Saudi Arabia (M Zamakhshary MD); Leibniz Institute for Prevention Research and
Epidemiology—BIPS, Bremen, Germany (Prof H Zeeb PhD); Chongqing Medical University, Chongqing, China (Prof Y Zhao MD); Zhongshan Ophthalmic Center, Sun Yat-sen University, Guangzhou, Guangdong, China (Y Zheng PhD); Zhejiang University School of Public Health, Hangzhou, Zhejiang, China (Prof S Zhu PhD); Landstuh Regional Medical Center, Landstuhl, USA (D Zonies MD); and Cancer Institute/Hospital, Chinese Academy of Medical Sciences, Beijing, Beijing, China (Prof X N Zou MD)

\section{Contributors}

ADL and CJLM conceived the study and provided overall guidance. CJLM, ADL, MN, and HW prepared the first draft. All other authors provided data, developed models, reviewed results, initiated modelling infrastructure, or reviewed and contributed to the report.

\section{Declaration of interests}

BDG works for AMP, which receives grant support for vaccine and immunisation related work from Crucell, GlaxoSmithKline, Merck, Novartis, Pfizer, and Sanofi Pasteur; however, none of this support is for work related to the present report. KJ reports has consulted for GlaxoSmithKline on projects outside the submitted work. WM is program analyst at the UNFPA country office in Peru, which does not necessarily endorse the study. JAS has received research grants from Takeda and Savient and consultant fees from Savient, Takeda, Regeneron, and Allergan. JAS is a member of the executive of OMERACT, which receives funding from 36 companies; a member of the American College of Rheumatology's Guidelines Subcommittee of the Quality of Care Committee; and a member of the Veterans Affairs Rheumatology Field Advisory Committee. RFG is associate editor of Annals of Epidemiology for which he receives a stipend. CK receives research grants from Brazilian public funding agencies Conselho Nacional de Desenvolvimento Científico e Tecnológico (CNPq), Coordenação de Aperfeiçoamento de Pessoal de Nível Superior (CAPES), and Fundação de Amparo à Pesquisa do Estado do Rio Grande do Sul (FAPERGS). He has also received authorship royalties from publishers Artmed and Manole. GR has consultancy agreements with Alexion Pharmaceuticals, Reata Pharmaceuticals, Bayer Healthcare, and Novartis Pharma, and is a member of the Abbvie Atrasentan Steering Committee; GR does not accept personal remuneration, compensations are paid to his institution for research and educational activities. MDH has received research support from the National Heart, Lung, and Blood Institute and World Heart Federation for its Emerging Leaders program, which is supported by unrestricted educational grants from AstraZeneca and Boehringer Ingelheim. FP-R has received investigation grants from Ministerio de Sanidad, Gobierno de España, Asociación de Reumatólogos del Hospital de Cruces, Fundación Española de Reumatología; has been a consultant (with or without payment) for Astra-Zeneca, Menarini, Metabolex, Ardea Biosciences, SOBI, Novartis, and Pfizer; and has been a speaker for AstraZeneca and Menarini. KBG received the NHMRC-Gustav Nossai scholarship sponsored by CSL Behring in 2013. MGS has previously served as consultant for Ethicon on global surgery. PJ is supported by a career development fellowship from the Wellcome Trust, Public Health Foundation of India, and a consortium of UK universities. DAQ was supported by The Eunice Kennedy Shriver National Institute of Child Health and Human Development of the National Institutes of Health (number 5T32HD057822). AK has received institutional support (intramural funding) from the Oklahoma State University Center for Health Sciences. RAL receives funding through the Farr Institute of Health Informatics Research. The Farr Institute is supported by Arthritis Research UK, British Heart Foundation, Cancer Research UK, Economic and Social Research Council, Engineering and Physical Sciences Research Council, Medical Research Council, National Institute of Health Research, National Institute for Social Care and Health Research (Welsh Government), and the Chief Scientist Office (Scottish Government Health Directorates), (MRC grant MR/K006525/1). DM reports ad hoc honoraria from Bunge, Pollock Institute, and Quaker Oats; ad hoc consulting for Foodminds, Nutrition Impact, Amarin, AstraZeneca, Winston and Strawn LLP, and Life Sciences Research Organization; membership of Unilever North America Scientific Advisory Board; and chapter royalties from UpToDate. RD and LB are employed by the US Department of Veterans Affairs. VC is on the speaker bureau for Boehringer Ingelheim Baker. MS is an employee of Novartis Pharma. All 
other authors declare no competing interests. The authors alone are responsible for the views expressed in this Article and they do not necessarily represent the views, decisions, or policies of the institutions with which they are affiliated.

\section{Acknowledgments}

We thank the countless individuals who have contributed to the Global Burden of Disease Study 2013 in various capacities. We acknowledge the extensive support from all staff members at the Institute for Health Metrics and Evaluation and specifically thank: Kelsey Pierce for her valuable guidance; James Bullard, Serkan Yalcin, Evan Laurie, and Andrew Ernst for their tireless support of the computational infrastructure required to produce the results; Linda A Ettinger for her expert administrative support; and Peter Speyer and Eden Stork for their persistent and invaluable work to gain access to and catalogue as much data as possible to inform the estimates. We also acknowledge the support of the Rwandan Ministry of Health's GBD Team, led by Agnes Binagwaho, for their collaboration and for reviewing the manuscript: Uwaliraye Parfait, Karema Corine, Jean Pierre Nyemazi, Sabin Nsanzimana, Yvonne Kayiteshonga, Marie Aimee Muhimpundu, Jean de Dieu Ngirabega, Ida Kankindi, Sayinzoga Felix, and Gasana Evariste. The following individuals acknowledge various forms of institutional support. RA-SS was funded by a UK MRC senior clinical fellowship. SB acknowledges additional funding or institutional support from International Development Research Center of Canada, Stanford University, and Rosenkranz Price for health-care research in developing countries. AR was supported by research grants from Brazilian research agencies CNPq and FAPEMIG. MK was supported by a NIDDK T32 grant through June 2014. RGN acknowledges that this work was supported in part by the Intramural Research Program of the National Institute of Diabetes and Digestive and Kidney Diseases. KK acknowledges the Government of India for giving him a University Grant Commission Junior Research Fellowship. GDT acknowledges support from NYU's US National Institute of Environmental Health Sciences Center grant (number ES00260). HC and SJL are supported by the intramural programme of $\mathrm{NIH}$, the National Institute of Environmental Health Sciences. KD is supported by a Wellcome Trust Fellowship in Public Health and Tropical Medicine (grant number 099876). HW, AF, HE, and FC are affiliated with the Queensland Centre for Mental Health Research, which receives funding from the Queensland Department of Health. LAR acknowledges the support of Qatar National Research Fund (04-924-3-251). TF is grateful to the Swiss National Science Foundation for an Early and an Advanced Postdoc Mobility fellowship (project number PBBSP3-146869 and P300P3-154634). IA acknowledges the UK National Institute for Health Research and the Medical Research Council for funding. HWH acknowledges support from Parnassia Psychiatric Institute, The Hague, Netherlands; the Department of Psychiatry, University of Groningen, University Medical Center Groningen, Netherlands; and the Department of Epidemiology, Columbia University, New York, NY, USA. JM has received support from the National Health and Medical Research Council John Cade Fellowship APP1056929. UM acknowledges funding from the German National Cohort Consortium. BOA acknowledges a Susan G. Komen for the Cure Research Program - Leadership Grant (number SAC110001). AJC's work on GBD was funded by Health Effects Institute and the William and Flora Hewlett Foundation. RD acknowledges that funding from the US Deptartment of Veterans Affairs supports his salary. RD acknowledges funding from the American Parkinson's Disease Association for support of this work. MK receives research support from the Academy of Finland, the Swedish Research Council, Alzheimer Association, and AXA Research Fund. SS receives postdoctoral funding from the Fonds de la recherche en santé du Québec. GA-C acknowledges funding and support from Health Sciences and Neurosciences (CISNEURO) Research Group, Cartagena de Indias, Colombia. No authors received additional compensation for their efforts.

References

1 Institute for Health Metrics and Evaluation. GBD 2013 Protocol: global burden of diseases, injuries, and risk factors. 2013. http:// www.healthdata.org/gbd/about/protocol (accessed Nov 3, 2014).

2 Wang H, Dwyer-Lindgren L, Lofgren KT, et al. Age-specific and sex-specific mortality in 187 countries, 1970-2010: a systematic analysis for the Global Burden of Disease Study 2010. Lancet 2012; 380: 2071-94.
3 Lozano R, Naghavi M, Foreman K, et al. Global and regional mortality from 235 causes of death for 20 age groups in 1990 and 2010: a systematic analysis for the Global Burden of Disease Study 2010. Lancet 2012; 380: 2095-128.

4 Salomon JA, Wang H, Freeman MK, et al. Healthy life expectancy for 187 countries, 1990-2010: a systematic analysis for the Global Burden Disease Study 2010. Lancet 2012; 380: 2144-62.

5 Salomon JA, Vos T, Hogan DR, et al. Common values in assessing health outcomes from disease and injury: disability weights measurement study for the Global Burden of Disease Study 2010. Lancet 2012; 380: 2129-43.

6 Vos T, Flaxman AD, Naghavi M, et al. Years lived with disability (YLDs) for 1160 sequelae of 289 diseases and injuries 1990-2010: a systematic analysis for the Global Burden of Disease Study 2010. Lancet 2012; 380: 2163-96.

7 Murray CJL, Vos T, Lozano R, et al. Disability-adjusted life years (DALYs) for 291 diseases and injuries in 21 regions, 1990-2010: a systematic analysis for the Global Burden of Disease Study 2010. Lancet 2012; 380: 2197-223.

8 Lim SS, Vos T, Flaxman AD, et al. A comparative risk assessment of burden of disease and injury attributable to 67 risk factors and risk factor clusters in 21 regions, 1990-2010: a systematic analysis for the Global Burden of Disease Study 2010. Lancet 2012; 380: 2224-60.

9 Lozano R, Gómez-Dantés H, Garrido-Latorre F, et al. Burden of disease, injuries, risk factors and challenges for the health system in Mexico. Salud Pública México 2013; 55: 580-94 [published in Spanish]

10 Yang G, Wang Y, Zeng Y, et al. Rapid health transition in China, 1990-2010: findings from the Global Burden of Disease Study 2010. Lancet 2013; 381: 1987-2015.

11 Murray C, Abraham J, Ali M. The state of US health, 1990-2010: Burden of diseases, injuries, and risk factors. JAMA 2013; 310: 591-606.

12 Murray CJ, Richards MA, Newton JN, et al. UK health performance: findings of the Global Burden of Disease Study 2010. Lancet 2013; 381: 997-1020.

13 Forouzanfar MH, Sepanlou SG, Shahraz S, et al. Evaluating causes of death and morbidity in Iran, global burden of diseases, injuries, and risk factors study 2010. Arch Iran Med 2014; 17: 304-20.

14 Naghavi M, Shahraz S, Sepanlou SG, et al. Health transition in Iran toward chronic diseases based on results of Global Burden of Disease 2010. Arch Iran Med 2014; 17: 321-35.

15 Shahraz S, Forouzanfar MH, Sepanlou SG, et al. Population health and burden of disease profile of Iran among 20 countries in the region: From Afghanistan to Qatar and Lebanon. Arch Iran Med 2014; 17: 336-42.

16 USAID. Global Health Programs: Progress Report to Congress FY 2012. Washington, DC: USAID, 2013.

17 WHO. Roadmap for Childhood Tuberculosis. 2013. http://apps. who.int/iris/bitstream/10665/89506/1/9789241506137_eng.pdf (accessed Nov 4, 2014).

18 UNAIDS. 2011-2015 Strategy: Getting to Zero. Joint United Nations Programme on HIV/AIDS, 2010. http://www.unaids.org/en/media/ unaids/contentassets/documents/unaidspublication/2010/JC2034_ UNAIDS_Strategy_en.pdf (accessed Nov 4, 2014).

19 Treatment Action Group (TAG). The Zero Declaration. July 22, 2012 New York, NY. http://www.treatmentactiongroup.org/tb/advocacy/ zero-declaration (accessed Nov 4, 2014).

20 Stop TB Partnership. No more crying, no more dying. Towards zero TB deaths in children. Genvea: World Health Organization, 2012 http://www.stoptb.org/assets/documents/news/ChildhoodTB_ report_singles.pdf (accessed Nov 4, 2014).

21 South Africa Info Reporter. South Africa's HIV/Aids battle plan SouthAfrica.info, 2012. http://www.southafrica.info/about/health/ aids-prevention.htm\#.UxZ1nfldXg9 (accessed Nov 4, 2014).

22 Ki-moon B. Secretary-General's message on World Malaria Day. United Nations, 2011. http://www.un.org/sg/statements/?nid=5219 (accessed Nov 4, 2014).

23 Department for International Development. UKAID. Towards zero infections: the UK's position paper on HIV in the developing world. 2011. https://www.gov.uk/government/uploads/system/uploads/ attachment_data/file/67523/twds-zero-infs-pos-paper-hiv-dev-wrld. pdf (accessed Nov 4, 2014).

24 Jamison DT, Summers LH, Alleyne G, et al. Global health 2035: a world converging within a generation. Lancet 2013; 382: 1898-955. 
25 WHO. Positioning health in the post-2015: WHO discussion paper. Geneva: World Health Organization, 2012. http://www.who.int/topics/ millennium_development_goals/post2015/WHOdiscussionpaper_ October2012.pdf (accessed Nov 4, 2014).

26 The world we want. Health in the post-2015 agenda: report of the global thematic consultation on health. 2013. http://www. worldwewant2015.org/health (accessed Nov 4, 2014).

27 Atun R, Jaffar S, Nishtar S, et al. Improving responsiveness of health systems to non-communicable diseases. Lancet 2013; 381: 690-67.

28 Institute for Health Metrics and Evaluation. The Global Burden of Disease: generating evidence, guiding policy. Seattle: IHME, 2013

29 James SL, Gubbins P, Murray CJ, Gakidou E. Developing a comprehensive time series of GDP per capita for 210 countries from 1950 to 2015. Popul Health Metr 2012; 10: 12

30 Gakidou E, Cowling K, Lozano R, Murray CJ. Increased educational attainment and its effect on child mortality in 175 countries between 1970 and 2009: a systematic analysis. Lancet 2010; 376: 959-74.

31 Ng M, Fleming T, Robinson M, et al. Global, regional, and national prevalence of overweight and obesity in children and adults during 1980-2013: a systematic analysis for the Global Burden of Disease Study 2013. Lancet 2014; 384: 766-81.

$32 \mathrm{Ng} \mathrm{M}$, Freeman MK, Fleming TD, et al. Smoking prevalence and cigarette consumption in 187 countries, 1980-2012. JAMA 2014; 311: 183-92.

33 Wang H, Liddell CA, Coates MM, et al. Global, regional, and national levels of neonatal, infant, and under- 5 mortality during 1990-2013: a systematic analysis for the Global Burden of Disease Study 2013. Lancet 2014; 384: 957-79.

34 Murray CJL, Rajaratnam JK, Marcus J, Laakso T, Lopez AD. What can we conclude from death registration? Improved methods for evaluating completeness. PLoS Med 2010; 7: e1000262.

35 Obermeyer Z, Rajaratnam JK, Park CH, et al. Measuring adult mortality using sibling survival: a new analytical method and new results for 44 countries, 1974-2006. PLoS Med 2010; 7: e1000260.

36 Kannisto V. Development of oldest-old mortality, 1950 1990: evidence from 28 developed countries. Odense University Press, 1994.

37 Gompertz B. On the nature of the function expressive of the law of human mortality, and on a new mode of determining the value of life contingencies. Philos Trans $R$ Soc Lond 1825; 115: 513-83.

38 Murray CJL, Ortblad KF, Guinovart C, et al. Global, regional, and national incidence and mortality for HIV, tuberculosis, and malaria during 1990-2013: a systematic analysis for the Global Burden of Disease Study 2013. Lancet 2014; 384: 1005-70.

39 Zellner A. An efficient method of estimating seemingly unrelated regressions and tests for aggregation bias. J Am Stat Assoc 1962; 57: $348-68$.

40 UNAIDS. Methodology-understanding the HIV estimates. Strategic Information and Monitoring Division, $2013 \mathrm{http}: / / w w w . u n a i d s . o r g /$ en/media/unaids/contentassets/documents/epidemiology/2013/ gr2013/20131118_Methodology.pdf (accessed Nov 4, 2014).

41 Department of Peace and Conflict Research. Uppsala Conflict Data Program. Uppsala Universitet, Uppsala, Sweden. http://www.pcr. uu.se/research/UCDP/ (accessed Nov 4, 2014).

42 The International Institute For Strategic Studies. Armed Conflict Database. London. https://acd.iiss.org/ (accessed Nov 4, 2014).

43 WHO. WHO Mortality Database. http://www.who.int/healthinfo/ cod/en/ (accessed May 5, 2014)

44 Centre for Research on the Epidemiology of Disasters (CRED), Office of US Foreign Disaster Assistance (OFDA). EM-DAT: The OFDA/CRED international disaster database. Brussels, Belgium, Université Catholique de Louvain. http://www.emdat.be/database (accessed Nov 4, 2014).

45 United Nations Department of Economics and Social Affairs Population Division. World Population Prospects: The 2012 Revision. http://esa.un.org/unpd/wpp/Documentation/ publications.htm (accessed Nov 4, 2014).

46 Wheldon MC, Raftery AE, Clark SJ, Gerland P. Reconstructing past populations with uncertainty from fragmentary data. J Am Stat Assoc 2013; 108: 96-110.

47 Murray CJ, Lozano R, Flaxman AD, et al. Using verbal autopsy to measure causes of death: the comparative performance of existing methods. BMC Med 2014; 12: 5
48 North AB, South CD. Cancer incidence in Antarctica (2003-2007). Forman D, Bray F, Brewster DH, et al, eds. Cancer Incidence in Five Continents, Vol X (electronic version). Lyon, IARC. http://ci5.iarc.fr (accessed 22 Nov, 2014).

49 Ahern RM, Lozano R, Naghavi M, Foreman K, Gakidou E, Murray CJ. Improving the public health utility of global cardiovascular mortality data: the rise of ischemic heart disease. Popul Health Metr 2011; 9: 8

50 WHO. International Statistical Classification of Diseases and Related Health Problems 10th Revision (ICD-10) Version for 2010. Geneva: World Health Organization. http://apps.who.int/ classifications/icd10/browse/2010/en\#/X59 (accessed May 5, 2014).

51 WHO. WHO evidence review group: malaria burden estimation. Report on the second meeting, geneva, Switzerland, 2013. http:// www.who.int/malaria/mpac/malaria_burden_estimates_report.pdf (accessed May 4, 2014).

52 Foreman KJ, Lozano R, Lopez AD, Murray CJ. Modeling causes of death: an integrated approach using CODEm. Popul Health Metr 2012; 10: 1 .

53 Ortblad KF, Lozano R, Murray CJL. The burden of HIV: insights from the Global Burden of Disease Study 2010. AIDS 2013; 27: 2003-17.

54 Murray CJL, Ezzati M, Flaxman AD, et al. GBD 2010: design, definitions, and metrics. Lancet 2012; 380: 2063-66.

55 Flaxman A, ed. Integrated meta-regression framework for descriptive epidemiology. University of Washington Press, 2014.

56 Blackwelder WC, Biswas K, Wu Y, et al. Statistical methods in the Global Enteric Multicenter Study (GEMS). Clin Infect Dis Off Publ Infect Dis Soc Am 2012 55 (suppl 4): S246-53.

57 Kotloff KL, Nataro JP, Blackwelder WC, et al. Burden and aetiology of diarrhoeal disease in infants and young children in developing countries (the Global Enteric Multicenter Study, GEMS): a prospective, case-control study. Lancet 2013; 382: 209-22.

58 Hanley JA. A heuristic approach to the formulas for population attributable fraction. J Epidemiol Community Health 2001; 55: 508-14.

59 Levine MM, Kotloff KL, Nataro JP, Muhsen K. The Global Enteric Multicenter Study (GEMS): impetus, rationale, and genesis. Clin Infect Dis Off Publ Infect Dis Soc Am 2012; 55 (suppl 4): S215-24

60 Kotila SM, Virolainen A, Snellman M, Ibrahem S, Jalava J, Lyytikäinen O. Incidence, case fatality and genotypes causing Clostridium difficile infections, Finland, 2008

Clin Microbiol Infect Off Publ Eur Soc Clin Microbiol Infect Dis 2011; 17: 888-93.

61 Klugman KP. Contribution of vaccines to our understanding of pneumococcal disease. Philos Trans R Soc Lond B Biol Sci 2011; 366: 2790-98.

62 Feikin DR, Scott JAG, Gessner BD. Use of vaccines as probes to define disease burden. Lancet 2014; 383: 1762-70.

63 Watt JP, Wolfson LJ, O'Brien KL, et al. Burden of disease caused by Haemophilus influenzae type $\mathrm{b}$ in children younger than 5 years: global estimates. Lancet 2009; 374: 903-11.

64 O'Brien KL, Wolfson LJ, Watt JP, et al. Burden of disease caused by Streptococcus pneumoniae in children younger than 5 years: global estimates. Lancet 2009; 374: 893-902.

65 Baqui AH, El Arifeen S, Saha SK, et al. Effectiveness of Haemophilus influenzae type B conjugate vaccine on prevention of pneumonia and meningitis in Bangladeshi children: a case-control study. Pediatr Infect Dis J 2007; 26: 565-71.

66 Gessner BD, Sutanto A, Linehan M, et al. Incidences of vaccine-preventable Haemophilus influenzae type b pneumonia and meningitis in Indonesian children: hamlet-randomised vaccine-probe trial. Lancet 2005; 365: 43-52.

67 Levine OS, Lagos R, Muñoz A, et al. Defining the burden of pneumonia in children preventable by vaccination against Haemophilus influenzae type b. Pediatr Infect Dis J 1999; 18: 1060-64.

68 Mulholland K, Hilton S, Adegbola R, et al. Randomised trial of Haemophilus influenzae type-b tetanus protein conjugate vaccine [corrected] for prevention of pneumonia and meningitis in Gambian infants. Lancet 1997; 349: 1191-97.

69 Black SB, Shinefield HR, Ling S, et al. Effectiveness of heptavalent pneumococcal conjugate vaccine in children younger than five years of age for prevention of pneumonia. Pediatr Infect Dis J 2002; 21: 810-15. 
70 Cutts FT, Zaman SMA, Enwere G, et al. Efficacy of nine-valent pneumococcal conjugate vaccine against pneumonia and invasive pneumococcal disease in The Gambia: randomised, double-blind placebo-controlled trial. Lancet 2005; 365: 1139-46.

71 Klugman KP, Madhi SA, Huebner RE, Kohberger R, Mbelle N, Pierce N. A trial of a 9-valent pneumococcal conjugate vaccine in children with and those without HIV infection. N Engl J Med 2003; 349: 1341-48.

72 Lucero MG, Nohynek H, Williams G, et al. Efficacy of an 11-valent pneumococcal conjugate vaccine against radiologically confirmed pneumonia among children less than 2 years of age in the Philippines: a randomized, double-blind, placebo-controlled trial. Pediatr Infect Dis J 2009; 28: 455-62.

73 Sigaúque B, Vubil D, Sozinho A, et al. Haemophilus influenzae type $b$ disease among children in rural Mozambique: impact of vaccine introduction. J Pediatr 2013; 163: S19-24.

74 Gessner BD, Adegbola RA. The impact of vaccines on pneumonia: key lessons from Haemophilus influenzae type b conjugate vaccines. Vaccine 2008; 26 (suppl 2): B3-8.

75 Simonsen L, Viboud C, Taylor RJ, Miller MA. The epidemiology of influenza and its control. In: Rappuoli R, Giudice GD, eds. Influenza vaccines for the future. Springer Basel, 2011: 27-54.

76 Griffin MR, Zhu Y, Moore MR, Whitney CG, Grijalva CG. U.S. Hospitalizations for pneumonia after a decade of pneumococcal vaccination. N Engl J Med 2013; 369: 155-63.

77 Gini C. Variabilità e mutabilità, contribrito allo studio delle distribuzioni e delle relazioni statistiche: fascicolo Ier: Introduzione-Indici di variabilita—Indici di mutabilità. Bologne, 1912.

78 Preston S, Heuveline P, Guillot M. Demography: measuring and modeling population processes, 1st edn. Malden, MA: Wiley-Blackwell, 2000.

79 Beltrán-Sánchez H, Preston SH, Canudas-Romo V. An integrated approach to cause-of-death analysis: cause-deleted life tables and decompositions of life expectancy. Demogr Res 2008; 19: 1323-50.

80 Ahmad O, Boschi-Pinto C, Agresti A. Age standardization of rates: a new WHO standard. WHO, 2001 http://www.who.int/healthinfo/ paper31.pdf (accessed May 5, 2014).

81 United Nations, Department of Economic and Social Affairs Population Division, Population Division. World Population Ageing 2013. http://www.un.org/en/development/desa/population/ publications/pdf/ageing/WorldPopulationAgeing2013.pdf (accessed Nov 28, 2014).

82 Mueller Y, Cristofani S, Rodriguez C, et al. Integrating mental health into primary care for displaced populations: the experience of Mindanao, Philippines. Confl Health 2011; 5: 3.

83 International Agency for Research on Cancer. GLOBOCAN 2012: Estimated cancer incidence, mortality and prevalence worldwide in 2012. WHO: International Agency for Reserach on Cancer. http:// globocan.iarc.fr/Default.aspx (accessed May 5, 2014).

84 UNICEF. Committing to child survival: a promise renewedprogress report 2013. New York: UNICEF, 2013.

85 USAID. Child survival: call to action. Ending preventable child deaths. Washington: USAID, 2012.

86 Bustreo F, Say L, Koblinsky M, Pullum TW, Temmerman M, Pablos-Méndez A. Ending preventable maternal deaths: the time is now. Lancet Glob Health 2013; 1: e176-e177.

87 Quah DT. Twin peaks: growth and convergence in models of distribution dynamics. Econ J 1996; 106: 1045-55.

88 Sala-i-Martin X. The world distribution of income: falling poverty and...convergence, period. O J Econ 2006; 121: 351-97.

89 Ravallion M. Why don't we see poverty convergence? Am Econ Rev 2012; 102: 504-23.

90 Bloom DE, Canning D. Mortality traps and the dynamics of health transitions. Proc Natl Acad Sci 2007; 104: 16044-49.

91 Clark R. World health inequality: convergence, divergence, and development. Soc Sci Med 2011; 72: 617-24.

92 Wilson C. On the scale of global demographic convergence 1950-2000. Popul Dev Rev 2001; 27: 155-71.

93 Vallin J, Meslé F. Convergences and divergences in mortality. Demogr Res Spec Collect 2004; 2: 11-44.

94 Moser K, Shkolnikov V, Leon DA. World mortality 1950-2000: divergence replaces convergence from the late $1980 \mathrm{~s}$. Bull World Health Organ 2005; 83: 202-09.
95 Balabanova D, Mills A, Conteh L, et al. Good Health at Low Cost 25 years on: lessons for the future of health systems strengthening. Lancet 2013; 381: 2118-33.

96 Jamison DT, Breman JG, Measham AR, et al, eds. Disease control priorities in developing countries, 2nd edn. Washington: World Bank, 2006.

97 Leon DA, Shkolnikov VM, McKee M, Kiryanov N, Andreev E. Alcohol increases circulatory disease mortality in Russia: acute and chronic effects or misattribution of cause? Int J Epidemiol 2010; 39: 1279-90.

98 AIWH: Field B. Rheumatric heart disease: all but forgotten in Australia except among Aboriginal and Torres Strait Islander people. Australian Insitute of Health and Welfare, 2004. http:// www.aihw.gov.au/WorkArea/DownloadAsset.aspx?id=6442453321 (accessed Nov 4, 2014).

99 Matthews FE, Arthur A, Barnes LE, et al. A two-decade comparison of prevalence of dementia in individuals aged 65 years and older from three geographical areas of England: results of the Cognitive Function and Ageing Study I and II. Lancet 2013; 382: 1405-12.

100 United States Department of Health and Human Services. Centers for Disease Control and Prevention. National Center for Health Statistics. National Mortality Followback Survey, 1993. Ann Arbor: Inter-university Consortium for Political and Social Research, 2000.

101 Nash TE, Herrington DA, Losonsky GA, Levine MM. Experimental human infections with Giardia lamblia. J Infect Dis 1987; 156: 974-84.

102 Musher DM, Musher BL. Contagious acute gastrointestinal infections. N Engl J Med 2004; 351: 2417-27.

103 Rendtorff RC. The experimental transmission of human intestinal protozoan parasites. II. Giardia lamblia cysts given in capsules. Am J Hyg 1954; 59: 209-20.

104 Yoder J, Harral C, Beach M, Division of Foodborne, Waterborne, and Environmental Diseases (proposed), National Center for Emerging and Zoonotic Infectious Diseases (proposed), CDC. Giardiasis Surveillance-United States, 2006-2008. Centers for Disease Control, 2010.

105 Laishram S, Kang G, Ajjampur SSR. Giardiasis: a review on assemblage distribution and epidemiology in India. Indian J Gastroenterol 2012; 31: 3-12.

106 Walker CLF, Rudan I, Liu L, et al. Global burden of childhood pneumonia and diarrhoea. Lancet 2013; 381: 1405-16.

107 Nair H, Nokes DJ, Gessner BD, et al. Global burden of acute lower respiratory infections due to respiratory syncytial virus in young children: a systematic review and meta-analysis. Lancet 2010; 375: 1545-55.

108 Nair H, Brooks WA, Katz M, et al. Global burden of respiratory infections due to seasonal influenza in young children: a systematic review and meta-analysis. Lancet 2011; 378: 1917-30.

109 Pfizer. Pfizer presents detailed results from landmark communityacquired pneumonia immunization trial in adults (CAPiTA) evaluating efficacy of prevenar 13. New York, NY, 2014. http://www. pfizer.com/news/press-release/press-release-detail/pfizer_presents_ detailed_results_from_landmark_community_acquired_ pneumonia_immunization_trial_in_adults_capita_evaluating efficacy_of_prevenar_13 (accessed Nov 4, 2014).

110 Levine OS, O'Brien KL, Deloria-Knoll M, et al. The pneumonia etiology research for child health project: a 21st century childhood pneumonia etiology study. Clin Infect Dis 2012; 54: S93-101.

111 Gunnell D, Eddleston M, Phillips MR, Konradsen F. The global distribution of fatal pesticide self-poisoning: systematic review. BMC Public Health 2007; 7: 357

112 Sahloul Z, Coutts A, Fouad FM, et al. Health response system for Syria: beyond official narrative. Lancet 2014; 383: 407.

113 Das S, Kolher RP, Mane BG, Singh JPN, Singh AP. Chikungunya epidemic: global and Indian scenario. J Commun Dis 2007; 39: $37-43$.

114 Kalantri SP, Joshi R, Riley LW. Chikungunya epidemic: an Indian perspective. Natl Med J India 2006; 19: 315-22.

115 Raheel U, Faheem M, Riaz MN, et al. Dengue fever in the Indian subcontinent: an overview. J Infect Dev Ctries 2011; 5: 239-47.

116 Dhama K, Verma AK, Rajagunalan S, et al. Swine flu is back again: a review. Pak J Biol Sci PJBS 2012; 15: 1001-09.

117 Forouzanfar MH, Foreman KJ, Delossantos AM, et al. Breast and cervical cancer in 187 countries between 1980 and 2010: a systematic analysis. Lancet 2011; 378: 1461-84. 
Articles

118 Hill K, Zimmerman L. Adolescent mortality in low- and middleincome countries. WHO, $2013 \mathrm{http}$ //globalhealth2035.org/sites/ default/files/working-papers/adolescent-mortality.pdf (accessed May 5, 2014).

119 Gore FM, Bloem PJ, Patton GC, et al. Global burden of disease in young people aged 10-24 years: a systematic analysis. Lancet 2011; 377: 2093-102.

120 Patton GC, Coffey C, Sawyer SM, et al. Global patterns of mortality in young people: a systematic analysis of population health data. Lancet 2009; 374: 881-92.

121 Brown GD, Denning DW, Levitz SM. Tackling human fungal infections. Science 2012; 336: 647.

122 Vangen S, Ellingsen L, Andersgaard AB, et al. Maternal deaths in Norway 2005-2009. Tidsskr Den Nor Laegeforening Tidsskr Prakt Med Ny Raekke 2014; 134: 836-39.
123 Roulson J, Benbow EW, Hasleton PS. Discrepancies between clinical and autopsy diagnosis and the value of post mortem histology; a meta-analysis and review. Histopathology 2005; 47: 551-59.

124 Sonderegger-Iseli K, Burger S, Muntwyler J, Salomon F. Diagnostic errors in three medical eras: a necropsy study. Lancet 2000; 355: 2027-31.

125 Murray CJL, Kulkarni SC, Ezzati M. Understanding the coronary heart disease versus total cardiovascular mortality paradox a method to enhance the comparability of cardiovascular death statistics in the United States. Circulation 2006; 113: 2071-81. 